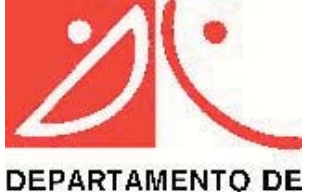
DEPARTAMENTO DE
COMUNICACIONES

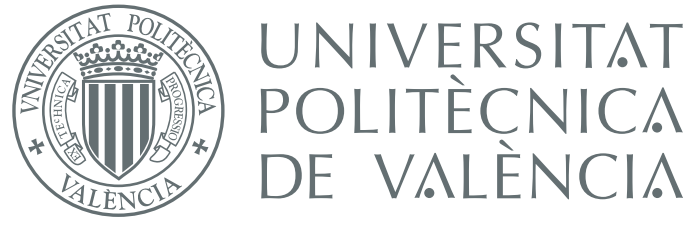

Universidad Politécnica de Valencia

Departamento de Comunicaciones

\title{
Discrete-time modelling of diffusion processes for room acoustics simulation and analysis.
}

\author{
Tesis Doctoral
}

presentada por

Juan Miguel Navarro Ruiz

dirigida por

Dr. José Escolano Carrasco

Prof. Dr. José Javier López Monfort

Valencia, Diciembre de 2011 


\section{DISCRETE-TIME MODELLING OF DIFFUSION PROCESSES FOR ROOM ACOUSTICS SIMULATION AND ANALYSIS.}

Submitted in partial fulfillment of the requirements for the degree of Doctor of Philosophy

by

Juan-Miguel Navarro-Ruiz

Technical University of Valencia

Valencia, Spain

December 2011 
Copyright (C) 2011 by Juan-Miguel Navarro-Ruiz. All rights reserved.

(1) $\emptyset$ 
To Ana Belen and Claudia.

I'm not a perfect person, but you are the pieces that complete our puzzle. 


\section{Abstract}

Sound propagation in an enclosed space is a complex phenomenon that depends on the geometrical properties of the room and the absorption features of the boundary's surface materials. The sound field's behaviour in rooms can be modelled using different theories, depending on the approach applied for describing the sound wave propagation.

This thesis focuses on room acoustics modelling in enclosed spaces using energy diffusion processes. In this work, how the diffusion equation theoretical model can simulate the sound field distribution in complex spaces is investigated.

The acoustic diffusion equation model has been actively studied in recent years, since it provides high efficiency and flexibility to the simulations of different types of enclosures; however, only a few research studies have been performed to deeply investigate the accuracy, advantages and limitations of this alternative method.

A systematic derivation of the acoustic diffusion equation method is developed, to establish the basis and assumptions of the model and to link it with the geometrical acoustics techniques. This also allows a proper description of its theoretical advantages and limitations.

This thesis is also devoted to the numerical implementation issues concerning the acoustic diffusion equation model. In this work, the sound field is modelled by means of finite-difference schemes. The results of this study provide practical and simple solutions by showing a low computational requirements in both time and memory consumption.

Finally, an evaluation of the acoustic diffusion equation model is carried out in order to study its performance for acoustic predictions in rooms. Special attention is paid to both temporal and spatial assumptions of the model. In these simulations, different scenarios and configurations are used to compare predicted values with measurement results and predictions from other well-established geometrical acoustics methods.

In general, the results show that the acoustic diffusion equation model can 
be used to predict room-acoustics time criteria, such as reverberation time, with accuracy. A deeper analysis reinforces the theoretical limitation that the diffusion equation is mainly valid for predicting the late part of the room impulse response. Moreover, it is observed that the spatial dependence of the predicted parameters with the diffusion equation is partially modelled, presenting low variability between several receiver positions within the room, as expected according to the theoretical assumptions.

Keywords: diffusion equation, radiative transfer equation, finite-difference scheme, room acoustics simulation. 
La propagación del sonido en una sala es un fenómeno complejo que depende de las propiedades geométricas del recinto y de las propiedades de absorción de los materiales que forman las superficies límite del mismo. El comportamiento del campo sonoro dentro de una sala puede modelarse mediante el uso de varias teorías dependiendo de la aproximación aplicada para describir la propagación de la onda de sonido.

Esta tesis se centra en el modelado de la acústica de recintos cerrados usando procesos de difusión de la energía. En este trabajo, se investiga como el modelo teórico de la ecuación de difusión puede simular la distribución del campo sonoro.

El modelo de la ecuación de difusión ha sido estudiado a lo largo de los últimos años de forma muy activa, dado que permite una alta flexibilidad y eficiencia en las simulaciones de diferentes tipos de salas; sin embargo, solo se han realizado unos pocos estudios de investigación sobre la precisión, ventajas y limitaciones de este método alternativo.

Para poder establecer las bases y las suposiciones del modelo, así como para enlazarlo con las técnicas de la acústica geométrica, se ha desarrollado una derivación sistemática del método de la ecuación de difusión acústica. Esto permite también una adecuada descripción de sus ventajas y limitaciones teóricas.

Esta tesis también esta dedicada a los detalles de implementación mediante métodos numéricos del modelo de la ecuación de difusión acústica. En este trabajo, el campo sonoro se ha modelado mediante esquemas de diferencias finitas. Los resultados de este estudio proporcionan soluciones simples y practicas que muestran unos requerimientos computacionales bajos tanto de consumo de memoria como de tiempo.

Finalmente, con el objeto de estudiar el rendimiento del modelo de la ecuación de difusión acústica en la predicción acústica de recintos se ha realizado evaluación del mismo. Se ha prestado especial atención a las suposiciones temporales y espaciales del modelo. En estas simulaciones se han utilizado diferentes escenarios y configuraciones de salas para comparar los 
resultados de las predicciones con medidas reales y con predicciones de otros métodos de la acústica geométrica bien establecidos.

En general, los resultados muestran que el modelo de la ecuación de difusión acústica puede utilizarse para predecir con precisión parámetros de calidad de acústica de salas, tales como el tiempo de reverberación. Un análisis mas profundo de los resultados permite reforzar la limitación teórica que afirma que la ecuación de difusión es principalmente valida para predecir la parte tardía de la respuesta al impulso de la sala. Además, como se esperaba de acuerdo con las suposiciones teóricas, se observa que la dependencia espacial de los parámetros obtenidos mediante la ecuación de difusión es parcialmente modelada, presentando una variabilidad baja entre los diferentes puntos receptores dentro de la sala.

Palabras clave: ecuación de difusión, ecuación de transferencia radiativa, esquema en diferencias finitas, simulación de la acústica de salas. 
La propagació del so en una sala és un fenomen complex que depén de les propietats geomètriques del recinte i de les propietats d'absorció dels materials que formen les superfícies límit del mateix. El comportament del camp sonor dins d'una sala pot modelar-se per mitjà de l'ús de diverses teories depenent de l'aproximació aplicada per a descriure la propagació de l'ona de so.

Esta tesi se centra en el modelatge de l'acústica de recintes tancats usant processos de difusió de l'energia. En este treball, s'investiga com el model teòric de l'equació de difusió pot simular la distribució del camp sonor.

El model de l'equació de difusió ha sigut estudiat al llarg dels últims anys de forma molt activa, atés que permet una alta flexibilitat i eficiència en les simulacions de diferents tipus de sales; no obstant això, només s'han realitzat uns pocs estudis d'investigació sobre la precisió, avantatges i limitacions d'este mètode alternatiu.

Per a poder establir les bases i les suposicions del model, així com per a enllaçar-ho amb les tècniques de l'acústica geomètrica, s'ha desenrotllat una derivació sistemàtica del mètode de l'equació de difusió acústica. Açò permet també una adequada descripció dels seus avantatges i limitacions teòriques.

Esta tesi també esta dedicada als detalls d'implementació per mitjà de mètodes numèrics del model de l'equació de difusió acústica. En este treball, el camp sonor s'ha modelat per mitjà d'esquemes de diferències finites.

Els resultats d'este estudi proporcionen solucions simples i practiques que mostren uns requeriments computacionals baixos tant de consum de memòria com de temps.

Finalment, amb l'objecte d'estudiar el rendiment del model de l'equació de difusió acústica en la predicció acústica de recintes s'ha realitzat avaluació del mateix. S'ha prestat especial atenció a les suposicions temporals i espacials del model. En estes simulacions s'han utilitzat diferents escenaris i configuracions de sales per a comparar els resultats de les prediccions amb 
mesures reals i amb prediccions d'altres mètodes de l'acústica geomètrica ben establits.

En general, els resultats mostren que el model de l'equació de difusió acústica pot utilitzar-se per a predir amb precisió paràmetres de qualitat d'acústica de sales, com ara el temps de reverberació. Una anàlisi mes profund dels resultats permet reforçar la limitació teòrica que afirma que l'equació de difusió és principalment valida per a predir la part tardana de la resposta a l'impuls de la sala. A més, com s'esperava d'acord amb les suposicions teòriques, s'observa que la dependència espacial dels paràmetres obtinguts per mitjà de l'equació de difusió és parcialment modelada, presentant una variabilitat baixa entre els diferents punts receptors dins de la sala.

Paraules clau: equació de difusió, equació de transferència radiativa, esquemes de diferències finites, simulació acústica de sales. 


\section{Abbreviation and Acronyms}

1-D

2-D

3-D

ARTE

BEM

DEM

DF

DWM

FDM

FDTD

FEM

FTCS

LTI

MLSSA

PDE

REM

RIR

TDS
One Dimensional

Two Dimensional

Three Dimensional

Acoustic Radiative Transfer Equation

Boundary Elements Method

Acoustic Diffusion Equation Model

Dufort Frankel Finite-Difference Scheme

Digital Waveguide Mesh

Finite-Difference Method

Finite-Difference Time-Domain

Finite Elements Method

Forward-Time Centred-Space Finite-Difference

Scheme

Linear Time Invariant

Maximum Length Sequence Signal Analysis

Partial Differential Equation

Room-acoustic Rendering Equation Model

Room Impulse Response

Time Delay Spectrometry 


\section{Notations and Conventions}

\section{Conventions}

The next conventions are used throughout this thesis:

- Time-domain scalar quantities are denoted by lowercase characters, e.g., $a(t)$.

- Frequency-domain scalar quantities are denoted by uppercase characters, e.g., $A(\omega)$.

- Time-domain vector quantities are denoted by boldface lowercase characters, e.g., $\mathbf{a}(t)$.

- Frequency-domain vector quantities are denoted by boldface uppercase characters, e.g., $\mathbf{A}(\omega)$.

- Time-domain matrix quantities are denoted by underlined, boldface lowercase characters, e.g., $\underline{\mathbf{a}}(t)$.

- Frequency-domain matrix quantities are denoted by lowercase characters, e.g., $\underline{\mathbf{A}}(\omega)$.

- Discretised vector or matrix are denoted by tilde characters, e.g., ã and $\underline{\tilde{a}}$ 


\section{Mathematical operations}

$(\cdot)^{T}$
$(\cdot)_{\mathrm{rms}}$
$(\cdot)^{*}$
$(\cdot)^{-1}$
$\mathbf{a} \cdot \mathbf{b}$
$\|\cdot\|$
$\int_{-\infty}^{t} f(\tau) d \tau$
$\mathcal{F}_{t}$
$\Im\{\cdot\}$
$\mathcal{L}$
$\partial / \partial t$
$\Re\{\cdot\}$
$\nabla$
$\nabla^{2}$
$*$
$\angle$

Vector or matrix transposition

Root mean square

Vector or matrix conjugated

Vector or matrix inverse

Dot product or scalar product of two vectors

$\mathrm{L}^{2}$ Norm or vector norm

Integration operator w.r.t. $t$

Time Fourier Transform

Imaginary component

Laplace Transform

Partial derivative w.r.t. $t$

Real component

Nabla operator (gradient)

Laplace operator

Convolution operator

Angular component of a complex number 


\section{List of symbols}

\section{Variables and constants}

$(\cdot)_{i}$

$(\cdot)_{r}$

$(\cdot)_{t}$

A

$A_{v}$

$A_{x}$

$\mathrm{C}_{80}$

D

$\mathrm{D}_{50}$

EDT

J

$L$

$L_{0}$

$L_{p}$

$L_{w}$

$L_{W}$

$N$

$N_{f}$

$P$

$R$

$R_{F}$

RT

$S_{\mathrm{i}}$

$S_{\text {o }}$

$S_{\mathrm{t}}$

$T$

$T_{\mathrm{amb}}$

$T_{F}$

TS

V

W
Incident component of the variable

Reflected component of the variable

Transmitted component of the variable

Equivalent absorption area of a room $\left(\mathrm{m}^{2}\right)$

Surface of a volume $\left(\mathrm{m}^{2}\right)$

Absorption factor

Clarity (dB)

Diffusion coefficient

Definition (\%)

Early decay time (s)

Sound intensity vector $\left(\mathrm{Wm}^{-2}\right)$

Sound radiance $\left(\mathrm{Wm}^{-2} \mathrm{Sr}^{-1}\right)$

Sound emittance $\left(\mathrm{Wm}^{-2}\right)$

Sound pressure level $(\mathrm{dB})$

Sound energy density level (dB)

Sound power level $(\mathrm{dB})$

Sound particle phase space density $\left(\mathrm{Jm}^{-3} \mathrm{sr}^{-1}\right)$

Number of room modes

Phase function $\left(\mathrm{sr}^{-1}\right)$

Reflection kernel

Reflection coefficient or surface scattering function $\left(\mathrm{sr}^{-1}\right)$

Reverberation time (s)

Area of each interior surface with different absorption coefficient $\left(\mathrm{m}^{2}\right)$

Area of each object with different absorption coefficient $\left(\mathrm{m}^{2}\right)$

Total interior surface area $\left(\mathrm{m}^{2}\right)$

Sampling period (s)

Ambient temperature $\left({ }^{\circ} \mathrm{C}\right)$

Transmission coefficient

Centre Time (s)

Volume of a space $\left(\mathrm{m}^{3}\right)$

Source sound power $(\mathrm{W})$ 
Specific acoustic impedance of a position in a

Z

$Z_{a}$

$c$

$d A$

$d V$

$d \Omega$

$d \Omega^{\prime}$

$f$

$f_{s}$

$f_{\text {limit }}$

$g$

$k$

$m$

$n$

$\hat{\mathbf{n}}$

$q$

$q_{0}$

p

$\mathbf{r}$

$\mathbf{r}_{b}$

$r_{c}$

$\mathbf{r}_{s}$

$\mathbf{r}=(\|\mathbf{r}\|, \theta, \phi)$

$\mathbf{r}=(x, y, z)$

$\tilde{\mathbf{r}}=(i, j, k)$

$s$

$\hat{\mathbf{s}}$

$\hat{\mathbf{s}}^{\prime}$

$t$

$\mathbf{u}$

$\hat{\mathbf{u}}$

$w$

$\Delta x, \Delta y, \Delta z$

$\Delta \nu$

$\Omega$

$\alpha$ medium (Rayl)

Characteristic acoustic impedance of a medium

(Rayl)

Speed of sound $\left(\mathrm{ms}^{-1}\right)$

Infinitesimal surface of a volume $\left(\mathrm{m}^{2}\right)$

Infinitesimal volume element $\left(\mathrm{m}^{3}\right)$

Differential solid angle (outgoing) (sr)

Infinitesimal solid angle (incoming) (sr)

Linear frequency $(\mathrm{Hz})$

Sampling frequency $(\mathrm{Hz})$

Schroeder's frequency $(\mathrm{Hz})$

Geometrical term

Wavenumber

Air attenuation $\left(\mathrm{m}^{-1}\right)$

Discrete time

Normal direction unit vector

Phase space source term $\left(\mathrm{Wm}^{-3} \mathrm{sr}^{-1}\right)$

Omnidirectional volume sound source $\left(\mathrm{Wm}^{-3}\right)$

Pressure (Pascal)

Position vector

Boundary position vector

Critical distance $(\mathrm{m})$

Source position vector

Continuous spherical coordinates

Continuous cartesian coordinates

Discrete cartesian coordinates

Source term

Direction unit vector (outgoing)

Direction unit vector (incoming)

Continuous time (s)

Particle or fluid velocity $\left(\mathrm{ms}^{-1}\right)$

Velocity unit vector

Sound energy density $\left(\mathrm{Jm}^{-3}\right)$

Discrete spatial steps (m)

$=[\Delta x, \Delta y, \Delta z]$

Solid angle (sr)

Absorption coefficient 
Mean-room absorption coefficient

$\theta_{r}$

$\lambda$

$\mu_{a}$

$\mu_{t}$

$\mu_{s}$

$\rho_{0}$

$\nu$

$\rho$

$\varphi$

$\psi$

$\omega$

\section{Special functions}

$\delta(n)$
$h(t)$
$x(t)$
$y(t)$
$\phi[u]$
$Y_{n, m}(x)$

Plane wave angle of incidence

Plane wave angle of reflection

Complex number, $\iota^{2}=-1$

Mean free path $(\mathrm{m})$

Absorption over a mean free path $\left(\mathrm{m}^{-1}\right)$

Attenuation over a mean free path $\left(\mathrm{m}^{-1}\right)$

Scattering over a mean free path $\left(\mathrm{m}^{-1}\right)$

Equilibrium density of the medium $\left(\mathrm{Kgm}^{-3}\right)$

$=[x, y, z]$

Visibility term

Density of the medium $\left(\mathrm{kgm}^{-3}\right)$

Wavelength $(\mathrm{m})$

Phase angle (r)

Angular frequency $\left(\mathrm{rs}^{-1}\right)$

Dirac Delta function

Impulse response function

Generic input signal of a system

Generic input signal of a system

Arbitrary function

Spherical harmonics of order $n$

and degree $m$, w.r.t. argument $x$ 


\section{Acknowledgments}

This research has been carried out at the Communication Department, Technical University of Valencia, between September 2008 and December 2011. I would like to express my gratitude to my supervisors Dr. José Escolano and José Javier López for letting me pursue a Ph.D. in this topic. I am deeply grateful for their dedicated and consistent support, guidance, and patience in my critical periods. Our almost daily discussions and their work-group policy greatly helped in the successful completion of this thesis. They have teach me not only how to be a scientist but also to be better man.

Many thanks go to my colleagues at the Audio and Communication Processing Group (GTAC), Máximo Cobos and Basilio Pueo, for creating a very vibrant and inspiring environment, with a family-like atmosphere. It has been a great pleasure to work with them, and after this thesis I hope we will keep working together. I am particularly grateful for your friendship in our moments around the world, and the very best social life which successfully provided me with numerous pleasant distractions from this work.

I have had the great honour of being a visiting Ph.D. student at the Department of Electrical Engineering, Acoustic Technology Group, Technical University of Denmark (DTU), in 2009. I am greatly indebted to Prof. Finn Jacobsen for a very inspirational stay at DTU, for many insightful discussions and for finding time to discuss my work despite the busy schedule. This summer time at Denmark was one of the best experience of my life, thanks José for convincing me. The work atmosphere was inspiring and those three months were a fundamental part of this thesis. Moreover, my 
wife and little daughter were there, she celebrated her first birthday, supporting me a lot, also travelling and having fun. I cannot forget to mention my office's colleagues Efren Fernandez and David Pelegrin, among other postgraduate students and staff, for their help during that period, affection and friendship. It was like being at home.

I want to express my gratitude to all the staff of my current Department (Telecommunication Engineering) at the San Antonio's University of Murcia. We share common goals trying to raise the research potential of the Department, and I sure we will do it. I would like to thank Prof. Michael Vorländer, Prof. Ning Xiang, Dr. Yun Jing, Prof. Lauri Savioja, Dr. CheolHo Jeong, and Dr. Jason E. Summer for insightful discussions related to my work on numerous occasions at our convention meetings or emails. Some of them are reviewers of this thesis improving its value and quality with their optimum comments.

Sincere thanks go to my wife, Ana Belen, for her love, continuous encouragement and sharing ups and downs related to the Ph.D. experience. Just I want to say to Claudia I love you. Last but definitely not least, I am indebted to my parents, and all my friends, for making me who I am and for being there for me. 


\section{Contents}

Abstract $\quad$ i

Abbreviation and Acronyms vii

$\begin{array}{ll}\text { Notations and Conventions } & \text { ix }\end{array}$

Acknowledgments $\quad$ xv

1 Introduction and Scope 1

1.1 Introduction . . . . . . . . . . . . . . . . 1

1.2 Motivation and scope of the thesis . . . . . . . . 2

1.3 Organization of the thesis $\ldots \ldots \ldots \ldots$

2 The Sound Field in an Enclosed Space 9

2.1 Introduction . . . . . . . . . . . . . . . 10

2.2 Sound waves propagation $\ldots \ldots \ldots \ldots$

2.2.1 Free field sound propagation . . . . . . . . . . . 11

2.2.2 Sound pressure and particle velocity . . . . . . . 12

2.2 .3 Plane waves . . . . . . . . . . . . . . 12 
2.2.4 Spherical waves . . . . . . . . . . . . 15

2.2 .5 Acoustic Impedance . . . . . . . . . . . . . . 17

2.2.6 Sound energy density and sound intensity . . . . . 18

2.2.7 Reflection and absorption of acoustic waves . . . . 20

2.2.7.1 Reflection .............. 20

2.2.7.2 Absorption . . . . . . . . . . . 22

2.3 Sound in an enclosed space $\ldots \ldots \ldots \ldots$

2.3.1 Sound propagation within rooms . . . . . . 26

2.3.1.1 The direct sound . . . . . . . . . . . . 28

2.3.1.2 Early reflections . . . . . . . . . . . 29

2.3.1.3 The reverberant sound . . . . . . . . . 30

2.3.1.4 The room impulse response . . . . . . . . 31

2.3.2 Room acoustics approaches . . . . . . . . . . . 34

2.3.2.1 Quasi-plane wave approach . . . . . . . 34

2.3.2.2 Statistical approach . . . . . . . . . 35

2.3.2.3 Wave equation approach . . . . . . . . 40

2.3.3 Room-acoustic quality parameters . . . . . . . 45

2.3.3.1 Reverberation time, RT . . . . . . . . 46

2.3.3.2 Early decay time, EDT . . . . . . . . 47

2.3.3.3 Clarity, $\mathrm{C}_{80} \ldots \ldots \ldots \ldots$. . . . . . 47

2.3.3.4 Definition, $\mathrm{D}_{50} \ldots \ldots \ldots \ldots$

2.3.3.5 Centre time, TS . . . . . . . . . . 48

2.3.3.6 Just noticeable differences, jnd . . . . . . 48

2.4 Summary . . . . . . . . . . . . . . . . . . . 49

3 Room Acoustics Modelling Techniques $\quad 51$ 
3.1 Introduction . . . . . . . . . . . . . . 51

3.2 Statistical methods . . . . . . . . . . . . . 52

3.3 Wave methods . . . . . . . . . . . . . . . . . . 54

3.3 .1 Frequency domain . . . . . . . . . . 55

3.3.1.1 Finite Element Method . . . . . . . . 56

3.3.1.2 Boundary Element Method . . . . . . . . 57

3.3 .2 Time Domain . . . . . . . . . . . . . . 58

3.3.2.1 Finite-Difference Time-Domain . . . . . . . 58

3.3.2.2 Digital Waveguide Mesh . . . . . . . . . 60

3.4 Geometrical methods . . . . . . . . . . . . . . . . . . 60

3.4.1 Ray-tracing . . . . . . . . . . . . . . 64

3.4 .2 Image-source . . . . . . . . . . . . . . . 65

3.4.3 Acoustical radiosity . . . . . . . . . . . . 67

3.4.4 Room-acoustic rendering equation . . . . . . 69

3.4.5 Acoustic diffusion equation model . . . . . . . . . 70

3.5 Discussion . . . . . . . . . . . . . . . . 72

3.5.1 About room acoustics simulation methods . . . . . 72

3.5.2 About diffuse reflection . . . . . . . . . . 73

3.5.3 About numerical techniques . . . . . . . . . 75

\section{The Radiative Transfer Model for Sound Field Modelling in Rooms $\quad 79$}

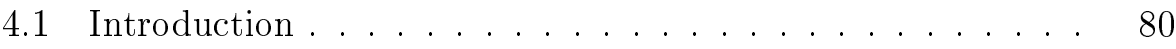

4.2 Mathematical phase space . . . . . . . . . . . . 81

4.3 Basic variables of the acoustic radiative transfer model . . . 82

4.4 Acoustic radiative transfer model . . . . . . . . . . . . . 86 
4.4.1 General assumptions . . . . . . . . . . . . 86

4.4.2 Governing acoustic radiative transfer equation . . . . 88

4.4.3 General boundary conditions . . . . . . . . . . 92

4.5 Derivation of the diffusion equation model from the acoustic radiative transfer equation . . . . . . . . . . . . 93

4.5.1 Technical and mathematical foundations of the acoustic diffusion equation model . . . . . . . . . . . 93

4.5.1.1 Governing diffusion equation . . . . . . . 94

4.5.1.2 Boundary conditions . . . . . . . . . . . 95

4.5.2 Relation to the acoustic radiative transfer model . . 97

4.5.2.1 Governing diffusion equation . . . . . . . 97

4.5.2.2 Boundary conditions . . . . . . . . . . . 101

4.6 Derivation of the room-acoustic rendering equation . . . . 103

4.6.1 Technical and mathematical foundations of the roomacoustic rendering equation . . . . . . . . . . 103

4.6.2 Relation to the acoustic radiative transfer model . . 104

4.7 Derivation of acoustical radiosity . . . . . . . . . . . . 109

4.7.1 Technical and mathematical foundations of acoustical radiosity . . . . . . . . . . . . . 109

4.7.2 Relation to the acoustic radiative transfer model . . 110

4.8 Discussion about assumptions, advantages and disadvantages 110

4.9 Summary . . . . . . . . . . . . . . . . . . . . 114

5 Implementation of a Diffusion Equation Model for Sound Field Modelling by a Finite-Difference Scheme 117

5.1 Introduction . . . . . . . . . . . . . . . . 117

5.2 Finite-difference schemes for the acoustic diffusion model . . 119 
5.2.1 Forward-time centred-space scheme . . . . . . . . . 120

5.2.1.1 Governing equation discretisation . . . . 120

5.2.1.2 Boundary conditions discretisation . . . . . 121

5.2.1.3 Von Neumman analysis . . . . . . . . . . . 124

5.2.1.4 Suitability . . . . . . . . . 126

5.2.2 Analysis of other schemes . . . . . . . . . . 127

5.2.3 Dufort-Frankel scheme . . . . . . . . . . . . . 129

5.2.3.1 Governing equation discretisation . . . . . 129

5.2.3.2 Boundary conditions discretisation . . . . . 129

5.2.3.3 Von Neumman analysis . . . . . . . . . 130

5.3 Evaluation of the implementations . . . . . . . . . 132

5.3.1 Spatial resolution . . . . . . . . . . . . . . 134

5.3 .2 Temporal resolution . . . . . . . . . . . 136

5.3.3 Computational efficiency . . . . . . . . . . . . . 139

5.3.4 Comparison with other methods . . . . . . . . . 141

5.4 Summary . . . . . . . . . . . . . . . . . . 144

6 Evaluation and Applicability of the Diffusion Equation Model $\begin{array}{ll}\text { for Room Acoustics Simulations } & 147\end{array}$

6.1 Introduction . . . . . . . . . . . . . . . . . . . 147

6.2 Simulations in rectangular rooms . . . . . . . . . . . . 149

6.2.1 Air absorption effects . . . . . . . . . . . . . 149

6.2.1.1 Introduction . . . . . . . . . . . . . 149

6.2.1.2 Analysis . . . . . . . . . . . . . . 149

6.2.2 Comparison with other diffuse reflection prediction models . . . . . . . . . . . . . . . . 152 
6.2.2.1 Introduction . . . . . . . . . . . 152

6.2.2.2 Analysis . . . . . . . . . . . . 153

6.2.3 Limitation in the temporal domain . . . . . . . . . 155

6.2.3.1 Introduction . . . . . . . . . . . . 155

6.2.3.2 Analysis . . . . . . . . . . . . . 156

6.3 Simulations in a complex shape room . . . . . . . . . . 163

6.3.1 Introduction . . . . . . . . . . . . 163

6.3 .2 Analysis . . . . . . . . . . . . . . 165

6.4 Summary . . . . . . . . . . . . . . . . 170

7 Conclusions and Future

$\begin{array}{ll}\text { Research Lines } & 177\end{array}$

7.1 Summary and conclusions . . . . . . . . . . . . 177

7.2 Contributions of this thesis f . . . . . . . . 178

7.3 Future research lines . . . . . . . . . . . . . . . . . 181

Bibliography 185 


\section{Introduction and Scope}

\subsection{Introduction}

IT IS WIDELY KNOWN THAT WORDS AND MUSIC ARE the most common means of communication among humans. Both languages produce information and sensations that are received by humans, who experience both their objective and their subjective aspects, e.g. the understanding of the message and the feeling of being surrounded by sound respectively [Beranek, $1954]$.

As in any communication system, the information transmission channel is critical for the proper exchange of messages. In acoustics, the room, as the environment where the sound is created and heard, can make important changes in the proper reception of the original message [Kuttruff, 4th edition, 2000].

The sound that we hear in any room is a combination of the direct sound that travels straight from the source to the receptor, and the indirect reverberant sound, the sound from the source that reflects off the walls, 
floor, ceiling or furniture before it reaches the receptor [Beranek, 1962].

The appropriate acoustic design of a room is very important because the physical properties of the enclosure must be optimal so that the message is altered as little as possible [Beranek, 1954]. Therefore, a prediction or simulation prior to enclosure construction could be very useful in socioeconomic terms, and it could also avoid costly building reforms.

In the past, room acoustics computer simulation has been a very active area of research, resulting in many software applications that have tried to improve the speed and accuracy of the results. Most of these computer programs are based on a mathematical approach using a model of wave propagation in a particular configuration, obtaining then the room impulse response [Vorländer, 2008]. Usually, these approaches for developing software applications with a certain degree of accuracy are based on wave and geometrical [Savioja, 1999] methods. Through the analysis of the simulation results, two main goals can be achieved. Firstly, as a prediction tool in the case of general purpose room acoustic design, the objective parameters such as reverberation time, clarity, definition, and others are obtained, allowing us to evaluate the room quality performance [Barron, 2000]. Secondly, as a reproduction tool of the spatial sound information through auralisation [Kleiner et al., 1990], that allows us to experience the impression of listening to the sound as if it was being played in a particular room.

\subsection{Motivation and scope of the thesis}

Room acoustics simulation connotes a physically-based simulation of sound appropriate for auralisation or the obtention of acoustic quality parameters. The task of such simulation is to model the interplay of sound among objects and walls of an environment to approximate the quantity and quality of sound reaching the ear of an observer. To properly simulate the sound in a room, for instance, the entire surroundings must be taken into account; architectural features and even the objects within it can affect the overall 
sound field [Kuttruff, 4th edition, 2000].

Among the different solutions appearing in the technical literature, this thesis deals with the computer simulation of acoustic spaces using geometrical acoustic models. In these methods a geometric optics analogy is used and it is assumed that sound waves behave as sound rays [Kuttruff, 4th edition, 2000]. Sound, just as light, is a wave phenomenon. There are several differences between light and sound including the slower propagation speed of sound, and wavelength size. Despite the differences, there are also several similarities between light and sound. Geometrical acoustics model assumes that the sound propagates in a straight line in a homogeneous medium. Under these considerations, the geometrical methods work accurately for high frequencies, since many low frequency phenomena cannot be inherently simulated, such as diffraction and wave interference.

There is a large number of geometrical methods with certain similarities but their theoretical foundations are often derived for each method separately [Savioja, 1999]. Therefore, there is a need for a general foundation for geometrical room acoustics modelling, especially when lately the scientific community has been paying special attention to new energy-based model paradigms, such as diffusion equation [Picaut et al., 1997] due to its high efficiency and flexibility.

The acoustic diffusion equation models the sound field in an enclosure with diffusely reflecting boundaries with a classical diffusion theory [Valeau et al., 2006], and it has been previously used for modelling heat transfer or particle diffusion. This approach has been applied to several singlespace enclosure types including rectangular rooms [Valeau et al., 2006], long rooms [Picaut et al., 1999b] and fitted rooms [Valeau et al., 2007], as well as coupled-spaces [Valeau et al., 2004, Billon et al., 2006, Xiang et al., 2009]. The diffusion equation models the distribution of acoustic energy density rather than sound pressure, which is governed by the wave equation. Furthermore, the acoustic diffusion equation is a parabolic differential equation while the wave equation is a hyperbolic differential equation. As a first ap- 
proximation, the main advantage is its capability to be applied regardless of the complexity of the room shape with a low computational cost. However, the acoustic diffusion equation model theoretical foundations and assumptions have to be clarified in order to classify it as a native geometrical model and to carry out proper comparisons with other geometrical methods.

Within the scope of this thesis, our effort is focused on the development of new theories associated with the radiative transfer equation and the diffusion equation. The relation between these two models is that, mathematically, diffusion arises as the long-time large-distance limit of the radiative transfer equation. Moreover, it will be shown that the other classical geometrical methods can be derived from this radiative transfer model. This is of relevance because it unifies these models and frames their limitations.

From the considerations above, the main goals proposed for this thesis emerges:

1. Acoustic radiative transfer equation: To contribute to the acoustic diffusion model by proposing a general acoustic energy propagation model using the classical radiative transfer theory from optics that unifies the foundations of a wide variety of geometrical acoustics methods. This general model must establish a direct link between geometrical acoustics and the diffusion equation and also consolidates the features of this alternative model.

2. Discrete-time domain methods for the acoustic diffusion equation: In previous technical literature, the diffusion equation model is numerically solved using discrete-frequency domain methods which does not inherently calculate the room impulse response. It would be desirable to develop a general time domain numerical approximation of the diffusion equation model for complex shape rooms in 3-D with an optimal finite-differences discretisation.

3. Simulation and analysis of room acoustics using the acoustic diffusion equation: The specific assumptions that define the acoustic diffusion 
model result in some theoretical advantages and limitations. To investigate these issues with a systematic research regarding the accuracy of the predicted sound field distribution in different room types. It would be of interest to analyse the temporal domain response and the spatial domain variability of these predictions.

In summary, the main scope of this thesis is

To contribute to the acoustic diffusion model by proposing a general room acoustics theory linking them with a wide variety of geometrical acoustics methods, unifying its foundations and clarifying its features. Moreover, to develop efficient time-domain algorithms for the acoustic diffusion equation model to adequately simulate and analyse room acoustics. These algorithms will be used to investigate the acoustic diffusion model's advantages and limitations.

\subsection{Organization of the thesis}

This thesis is organized as follows:

- Chapter 2: This chapter provides background information on the behaviour of sound propagation in free and enclosed space and how its properties can be quantified and analysed. Special attention is paid to the fundamental aspects of room acoustics theory. These notions are necessary for the proper comprehension of this dissertation; however, a reader with some experience in this field could just overview this chapter.

- Chapter 3: Since the mathematical description of sound distribution in a room is an inhomogeneous boundary value problem, the solution of realistic enclosures must be obtained through techniques that simplify these calculations. The purposes of this chapter are to introduce 
and revise the main techniques currently applicable to room acoustics modelling. The suitability of the so-called geometrical acoustics methods for room acoustics simulation is discussed, concluding that a proper general basis is needed, which is related to the contributions developed in Chap. 4. Furthermore, a brief review of recently presented alternative geometrical methods is included, the acoustic diffusion equation and the room acoustic rendering equation.

- Chapter 4: In this chapter, the first group of original contributions is presented. A theory is developed for acoustic radiative transfer modelling providing a solid foundation for a wide variety of geometrical acoustic algorithms including the most recent. From the acoustic radiative transfer model both the diffusion equation and the acoustic rendering equation are derived, and this is a significant contribution in that it unifies these models and frames their limitations. The derivation of the diffusion equation is particularly able to offer insight. A discussion about the theoretical advantages and limitation of each method finalises the chapter.

- Chapter 5: The second group of original contributions is based on the implementation of the acoustic diffusion model using finite-difference methods. First, an investigation is carried out to provide a suitable finite-difference scheme to implement the acoustic diffusion equation model, followed by a detailed evaluation of the behaviour of these algorithms.

- Chapter 6: The proposed finite-difference implementations in Chap. 5 are used to analyze the performance of the acoustic diffusion equation model. The theoretical advantages and limitations discussed in Chap. 4 are evaluated. The evaluation is done by comparison with other well-known simulation methods and measurement data. Numerical and experimental results are demonstrated.

- Chapter 7: Finally, conclusions obtained in this dissertation are pre- 
sented, including some guidelines for future research, opened by this thesis. 


\section{The Sound Field in an Enclosed Space 2}

The SOUND WAVE PROPAGATION IN A ROOM is a complex phenomenon that involves several interaction events between the sound wave and the enclosed space's elements. An enclosed space is defined as an acoustic environment that is bounded by physical objects such as walls, floor and ceiling, differentiating it from the situation existing outdoors in a free field.

The purpose of this chapter is to provide background information on the behaviour of sound propagation in free and enclosed spaces and how its properties can be quantified and analysed. In this chapter, some of the fundamental aspects of room acoustics theory are revisited and they form the physical basis for the contributions addressed in this thesis. 


\subsection{Introduction}

A sound is said to exist if a disturbance propagated through an elastic material causes an alteration in pressure or a displacement of the particles of the material, which can be detected by a person or by an instrument [Beranek, 1954]. The nature of sound propagation in gases can be described using the physical properties of such media. Room acoustics is defined as the propagation phenomena where acoustic waves travel through a gas, more particularly air, and interact with the limits of an enclosure, giving a particular sound pressure distribution [Kinsler et al., 2000]. Also, sound source properties have a main role in the final acoustic features of the room, and in its sound perception.

The present chapter deals with the physical foundations for sound wave propagation in acoustics, forming a solid base for understanding the following chapters. It is organized as follows: Sec. 2.2 deals with the definitions of the basic acoustic concepts involved in the sound propagation phenomena. In Sec. 2.3, a brief description of the sound propagation in enclosures key concepts for this thesis is presented, giving special attention to the different approaches to describe the sound propagation within rooms. Also, the relevance of the impulse response and the room-acoustic parameters in objectively describing the acoustic characteristics of a room are presented. Finally, this chapter is summarized.

\subsection{Sound waves propagation}

This section introduces the linear acoustic basis of sound propagation. Sound waves are described as compressional oscillatory disturbances that propagate in a fluid [Jacobsen, 2007] or as a result from time-varying perturbation of the dynamic and thermodynamic variables that describe the medium [Pierce, 2007].

In Sec. 2.2.1, the equation that describes the sound wave propagation 
is presented. This equation provides a relation between the basic acoustic variable pressure, and particle (or fluid) velocity (Sec. 2.2.2). Then the basic solutions of the wave equation, the plane wave (Sec. 2.2.3) and the spherical wave (Sec. 2.2.4), are introduced. The acoustic impedance and the quantities that take into account the flow of energy are then explained. Finally, the reflection and the absorption phenomena of sound are enunciated as an important feature of the sound propagation in enclosed spaces as detailed in Sec. 2.3.

\subsubsection{Free field sound propagation}

Firstly, some assumptions have to be made in order to explain the fundamental acoustic concepts. Initially, it is presupposed that the sound propagation is in a free field without atmospheric attenuation, i.e. unbounded in all directions. Furthermore, a homogeneous medium at rest with an equilibrium density $\rho_{0}$ and a static pressure $p_{0}$ of the medium is assumed. In accordance to these assumptions, the speed of sound $c$ is constant with reference to time and space. For air, its magnitude is calculated as [Everest, 2001]

$$
c=331.4 \sqrt{1+\frac{T_{\mathrm{amb}}}{273.15}}
$$

and it is approximated as follows [Kuttruff, 4th edition, 2000]:

$$
c=331.4+0.6 T_{\mathrm{amb}},
$$

where $T_{\mathrm{amb}}$ is the ambient temperature in centigrade ${ }^{\circ} \mathrm{C}$ and $c$ is measured in meters per second $\left(\mathrm{ms}^{-1}\right)$. Although in large halls where some gradient of temperature could exists, it is common practice to assume a constant speed of sound of approximately $343 \mathrm{~ms}^{-1}$ for a room temperature of $20^{\circ} \mathrm{C}$. Under these ambient conditions the density of air is $1.204 \mathrm{kgm}^{-3}$ and the static pressure is $101.3 \mathrm{kPa}$. Note that the speed of sound of a gas depends only on the temperature, not on the static pressure.

The basic laws for sound propagation in fluids are the conservation of mass and the Euler's equation of motion [Morse and Ingard, 1968]. Sound 
propagation as a wave motion in a fluid, and the effects of such wave motion can be mathematically described by an equation that states the facts that the mass is conserved, the longitudinal force caused by a difference in the local pressure is balanced by the inertia of the medium, and sound is very nearly an adiabatic phenomenon, that is, there is no flow of heat [Jacobsen, 2007]. This simplified equation is the linearised wave equation. It is a second-order partial differential equation that describes a longitudinal sound pressure wave propagating in a Cartesian coordinate system,

$$
\nabla^{2} p(\mathbf{r}, t)-\frac{1}{c^{2}} \frac{\partial^{2} p(\mathbf{r}, t)}{\partial t^{2}}=s(\mathbf{r}, t)
$$

where $p(\mathbf{r}, t)$ is the sound pressure given at position $\mathbf{r}$ and at instant $t$, the operator $\nabla^{2}$ is the Laplacian sum of the second derivatives with respect to the three cartesian coordinates, and $s(\mathbf{r}, t)$ models a sound source.

\subsubsection{Sound pressure and particle velocity}

The sound pressure is the difference between the instantaneous value of the total pressure and the static pressure $p_{0}$, and it is the quantity we hear [Pierce, 1994]. In general, the pressure $p$ is the force over an area applied on an object in a direction perpendicular to the surface; it is a scalar quantity, and it has SI units of pascals: $1 \mathrm{~Pa}=1 \mathrm{Nm}^{-2}$.

The particle velocity $\mathbf{u}$, measured in $\mathrm{ms}^{-1}$ as SI units, is the velocity of a particle (real or imaginary) in a medium as it transmits a wave [Pierce, 1994]; when applied to a sound wave through a medium of air, particle velocity would be the physical speed of an air molecule as it moves back and forth in the direction where the sound wave is travelling as it passes. Since the particle velocity is a vector, it is defined as

$$
\mathbf{u}=u_{x} \hat{\mathbf{x}}+u_{y} \hat{\mathbf{y}}+u_{z} \hat{\mathbf{z}} .
$$

\subsubsection{Plane waves}

Since the sound propagation is modelled as a wave equation, a set of basic solutions to these equations (waves) exists (see Eq. (2.3)), which are inter- 
preted as travelling disturbances through a medium. One of these basic solutions is the plane wave, which plays a major role in room acoustics simulation, as will be seen later in this thesis. The plane wave is defined when all the acoustic variables vary with time and with some cartesian coordinate $\|\mathbf{r}\|=r$ where $\|\cdot\|$ is the $\mathrm{L}^{2}$ norm or the vector norm, but are independent of position along planes normal to the $r$ direction, $p=p(r, t)$. Because $\nabla p$ has only the $r$ component, the fluid acceleration $\partial \mathbf{u} / \partial t$ must be in the $\pm r$ direction and if the initial particle velocity has no initial components in the transverse direction to $r$, these components will remain as zero. If one writes $\mathbf{u}(\mathbf{r}, t)=u(r, t) \hat{\mathbf{u}}$, where $\hat{\mathbf{u}}$ is the unit direction of increasing distance $r$ and in accordance to these simplifications, then the wave equation in its 1-D form can be obtained [Beranek, 1954]

$$
\frac{\partial^{2} p(r, t)}{\partial r^{2}}-\frac{1}{c^{2}} \frac{\partial^{2} p(r, t)}{\partial t^{2}}=0
$$

or equivalently

$$
\left(\frac{\partial}{\partial r}-\frac{1}{c} \frac{\partial}{\partial t}\right)\left(\frac{\partial}{\partial r}+\frac{1}{c} \frac{\partial}{\partial t}\right) p(r, t)=0
$$

The general solution to the latter equation is a sum of a function of $(t-r / c)$ and of a function of $(t+r / c)$, that is,

$$
p(r, t)=f(t-r / c)+g(t+r / c),
$$

where the function $f$ and $g$ are arbitrary [Everest, 2001]. This solution to the wave equation is known as the D'Alembert solution. A physical interpretation of a travelling wave using the D'Alembert solution is shown in Fig. 2.1.

The special case of a harmonic plane progressive wave is of great importance. Harmonic waves are generated by sinusoidal sources. For example, a harmonic plane wave propagating in the $r$-direction can be written,

$$
p(r, t)=p_{m} \sin (\omega t-k r+\psi)
$$




\section{The Sound Field in an Enclosed Space}

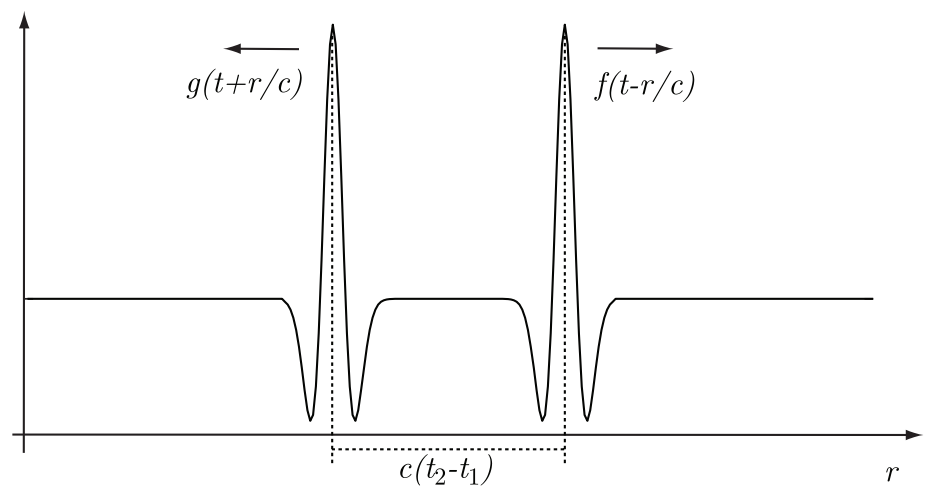

Figure 2.1. Physical interpretation of a 1-D travelling wave based on D'Alembert solution.

where $\omega=2 \pi f$ is the angular frequency and $k=\omega / c$ is the wavenumber. The quantity $p_{m}$ is the amplitude of the wave, and $\psi$ is the phase angle. The sound field at a fixed position varies sinusoidally with a number of oscillations per second $(\mathrm{Hz})$, known as linear frequency $f$. Moreover, the spatial period $\varphi$ indicates the sound pressure sinusoidal variations at a fixed time with $r$,

$$
\varphi=\frac{c}{f}=\frac{2 \pi c}{\omega}=\frac{2 \pi}{k}
$$

which is called wavelength as the distance travelled by the wave in one cycle.

Sound fields are often studied frequency by frequency. This allows the introduction of the complex exponential representation, where the sound pressure is written as a complex function of the position multiplied by a complex exponential taking into account the amplitude, phase and time dependence,

$$
p(r, t)=p_{m} e^{j(\omega t-k r)}
$$

The Euler's equation on motion [Morse and Feshbach, 1953] shows that the particle velocity is proportional to the gradient of the pressure. Using 
the complex representation of the pressure quantity in Eq. (2.10), it yields,

$$
u(r, t)=-\frac{1}{j \omega \rho_{0}} \frac{\partial p}{\partial r}=\frac{k}{\omega \rho_{0}} p_{m} e^{j(\omega t-k r)}=\frac{p_{m}}{\rho_{0} c} e^{j(\omega t-k r)}=\frac{p(r, t)}{\rho_{0} c} .
$$

The general solution to the $1-\mathrm{D}$ wave equation, Eq. (2.5), using the complex exponential representation is

$$
p(r, t)=p_{i} e^{j(\omega t-k r)}+p_{r} e^{j(\omega t+k r)},
$$

which can be defined as the sum of a wave that travels in the positive $\mathrm{x}$-direction and a wave that travels in the opposite direction (see Eq. (2.7)).

The corresponding expression for the particle velocity becomes, from Eq. (2.11)

$$
u(r, t)=\frac{p_{i}}{\rho_{0} c} e^{j(\omega t-k r)}-\frac{p_{r}}{\rho_{0} c} e^{j(\omega t+k r)} .
$$

\subsubsection{Spherical waves}

In addition to plane waves, another idealisation of wave propagation corresponds to a symmetric wave spreading in an unbounded fluid medium [Kinsler et al., 2000]. The source is considered to be a sphere with radius $a$ centred at the origin as Fig. 2.2 shows.

Since a spherical wave varies symmetrically according to $r$, which is the radius distance from the origin, a simple way to derive the wave shape of a spherical wave consists on expressing the wave equation into spherical coordinates $(r, \theta, \phi)$. After some modifications [Pierce, 1994], the wave equation in spherical coordinates is

$$
\frac{1}{r} \frac{\partial^{2}(r p(r, t))}{\partial r^{2}}-\frac{1}{c^{2}} \frac{\partial^{2} p(r, t)}{\partial t^{2}}=0
$$

where the pressure $p(r, t)$ has no dependence on $\theta$ or $\phi$. In a similar fashion to the plane wave, the wave solution to this equation is deduced as

$$
p(r, t)=r^{-1} f(t-r / c)+r^{-1} g(t+r / c),
$$

where $f$ and $g$ are a priori arbitrary functions. 


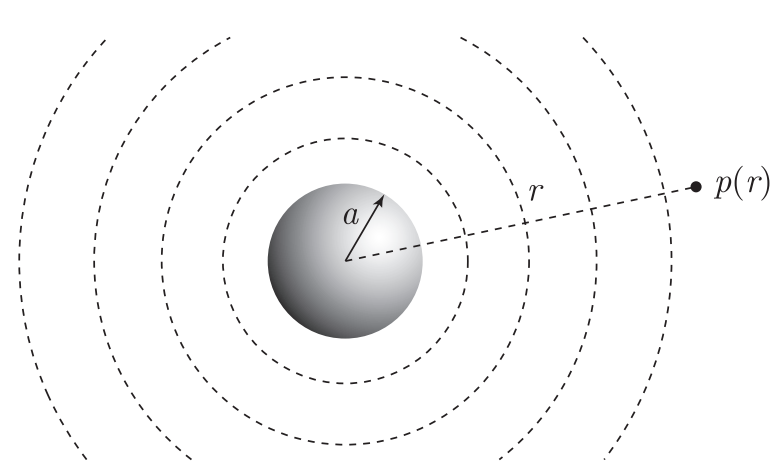

Figure 2.2. Vibrating spherical source with radius a radiating in a free field.

Outside of the region where the initial conditions are confined, if there are not any sources except for the one situated at the origin, waves only move in the $+r$ direction and thus, $g(t+r / c)=0$ (see Fig. 2.2).

A harmonic spherical wave is expressed in complex notation as follows,

$$
p(r, t)=p_{m} \frac{e^{j(\omega t-k r)}}{r} .
$$

The particle velocity in a spherical wave is not directly obtained as in the plane wave case, since particle velocity and pressure are not proportional. After using some concepts which are far from the scope of this thesis (see [Jacobsen, 2007] for details) the particle velocity is defined as

$$
u(r, t)=\frac{p(r, t)}{\rho_{0} c}\left(1+\frac{1}{j k r}\right) .
$$

An interesting conclusion of Eq. (2.17) arises. The peak values in time of the second term in the sum decrease with distance as $1 / r^{2}$ while the ones from the first term decrease as $1 / r$. This means that for a large $r$, the second term may be considered negligible compared with the first; then, the asymptotic relation between pressure and particle velocity gives the same relation as in plane waves. For waves of constant frequency, this relation is true when $r$ is much larger than its associate wavelength $(\simeq 10 \varphi)$. 


\subsubsection{Acoustic Impedance}

The specific acoustic impedance is a feature of a medium at a position in which the wave is propagating. The acoustic impedance is defined as the ratio of the sound pressure and the particle velocity at a position and a frequency,

$$
Z(\mathbf{r}, \omega)=\frac{P(\mathbf{r}, \omega)}{\mathbf{U}(\mathbf{r}, \omega) \cdot \hat{\mathbf{n}}} .
$$

For a progressive plane wave in the air, considering that the equations of the pressure Eq. (2.10) and the velocity Eq. (2.11) are in phase yields,

$$
Z_{a}=\rho_{0} c
$$

then the impedance of a progressive plane wave is independent of the particle position and the considered time. Eq. (2.19) is called the characteristic impedance of the medium and is a measure of the opposition to the motion of the particles within the medium. Eq. (2.19) has $\mathrm{Kg} \mathrm{m}^{-2} \mathrm{~s}^{-1}$ dimensions that is called Rayl in SI. The characteristic impedance of air at $20^{\circ} \mathrm{C}$ and $101.3 \mathrm{kPa}$ is about 413 Rayls.

For a progressive spherical wave, Eq. (2.17) shows that the relation between the sound pressure and the particle velocity do not remain constant but it depends on the distance $r$ and the angular frequency $\omega$. From Eq. (2.17) we get

$$
Z(\mathbf{r}, \omega)=\frac{P(r, \omega)}{\mathbf{U}(\mathbf{r}, \omega) \cdot \hat{\mathbf{n}}}=\frac{\rho_{0} c}{1+\frac{1}{j k r}}
$$

which implies that between $p$ and $\mathbf{u}$ there is a phase difference.

For $k r \gg 1$, i.e. for long distance comparing with the wavelength, Eq. (2.20) asymptotically tends to $\rho_{0} c$ which corresponds to the characteristic impedance of the medium. In this case, each element of the wavefront surface can be considered as an element of a plane wave. 


\subsubsection{Sound energy density and sound intensity}

Sound pressure is the basic and most important quantity for describing a sound field. However, generation and propagation of sound imply flow of energy that also provides fundamental information about sound field behaviour. Thus, sources of sound emit sound power to the medium, and sound waves carry energy along the medium [Jacobsen, 2007].

The acoustic wave propagation theory is based on conservation of energy. Hence, an energy balance model is used in linear acoustics [Pierce, 1994]. It can be enunciated as,

$$
\frac{\partial w(\mathbf{r}, t)}{\partial t}+\nabla \cdot \mathbf{J}(\mathbf{r}, t)=0
$$

where $w(\mathbf{r}, t)$ is the sound energy density and $\mathbf{J}(\mathbf{r}, t)$ is the instantaneous sound intensity.

Equation (2.21) is the conservation of sound energy, which expresses the simple fact that the rate of decrease of the sound energy density at a given position in a sound field $(-\partial w(\mathbf{r}, t) / \partial t)$ is equal to the rate of the flow of sound energy diverging away from the source point $(\nabla \cdot \mathbf{J}(\mathbf{r}, t))$.

Sound energy density $w(\mathbf{r}, t)$, measured in $\mathrm{Jm}^{-3}$, is an important concept in acoustics because, in dealing with sound in enclosures, it is necessary to study the flow of energy from a source to all parts of the room. The total instantaneous energy density is composed of the kinetic and potential energies per unit volume of gas [Everest, 2001],

$$
w(\mathbf{r}, t)=\frac{1}{2} \rho_{0}\|\mathbf{u}(\mathbf{r}, t)\|^{2}+\frac{1}{2} \frac{p^{2}(\mathbf{r}, t)}{\rho_{0} c^{2}} .
$$

Otherwise, the instantaneous sound intensity at a given position is the product of the instantaneous sound pressure and the instantaneous particle velocity [Everest, 2001],

$$
\mathbf{J}(\mathbf{r}, t)=\mathbf{u}(\mathbf{r}, t) p(\mathbf{r}, t) .
$$


This quantity, which is a vector, expresses the magnitude and direction of the instantaneous flow of sound energy per unit area at the given position, or the work done by the sound wave per unit area of an imaginary surface perpendicular to the vector.

In practice the time-averaged energy densities ${ }^{1}$,

$$
w(\mathbf{r})=\frac{1}{2} \rho_{0}\left\|\mathbf{u}_{\mathrm{rms}}(\mathbf{r}, t)\right\|^{2}+\frac{1}{2} \frac{p_{\mathrm{rms}}^{2}(\mathbf{r}, t)}{\rho_{0} c^{2}},
$$

are more important than the instantaneous quantities, and the time-averaged sound intensity

$$
\mathbf{J}(\mathbf{r})=\mathbf{J}_{\mathrm{rms}}(\mathbf{r}, t)=(\mathbf{u}(\mathbf{r}, t) p(\mathbf{r}, t))_{\mathrm{rms}}
$$

is more important than the instantaneous intensity $\mathbf{J}(\mathbf{r}, t)$.

The intensity vector in a harmonic plane wave is reduced, according to Eq. (2.11), as follows [Jacobsen, 2007]

$$
\mathbf{J}(\mathbf{r})=\frac{p_{\mathrm{rms}}^{2}(r)}{\rho_{0} c} \hat{\mathbf{n}}=c w(r) \hat{\mathbf{n}},
$$

where the kinetic and potential energies are therefore equal for plane waves.

In the harmonic spherical waves case, the intensity vector, according to Eq. (2.17), becomes the same relation for the radial sound intensity [Jacobsen, 2007]

$$
\mathbf{J}(r)=\frac{|p(r)|^{2}}{2 \rho_{0} c} \hat{\mathbf{n}}=\frac{p_{\mathrm{rms}}^{2}(r)}{\rho_{0} c} \hat{\mathbf{n}}
$$

It is apparent that there is a simple relation between the sound intensity and the mean square sound pressure in these two extremely important cases. However, it should be emphasised that in the general case Eq. (2.26) is

\footnotetext{
${ }^{1}$ the root mean square (abbreviated rms), also known as the quadratic mean, is a statistical measure of the magnitude of a varying quantity

$$
p_{\mathrm{rms}}^{2}(\mathbf{r})=\frac{1}{T_{2}-T_{1}} \int_{T_{1}}^{T_{2}} p^{2}(\mathbf{r}, t) d t
$$
}


not valid, and one will have to measure both the sound pressure and the particle velocity simultaneously and also average the instantaneous product over time in order to measure the sound intensity [Jacobsen, 2007].

A sound is created by a sound source, e.g, a musical instrument, people talking or a stereo system. The radiated sound power produced by a source is denoted by $W$ and is the rate at which sound energy is produced versus time measured in watts. It can be computed as

$$
W(\mathbf{r})=\int_{A_{v}} \mathbf{J}(\mathbf{r}) d A
$$

\subsubsection{Reflection and absorption of acoustic waves}

\subsubsection{Reflection}

When an acoustic wave is propagating in a medium and impacts on a boundary surface between two environments with infinite length, two new waves are generated. As Fig. 2.3 shows, one is reflected, and is propagated in the first medium, and the other is transmitted, and is propagated in the second medium.

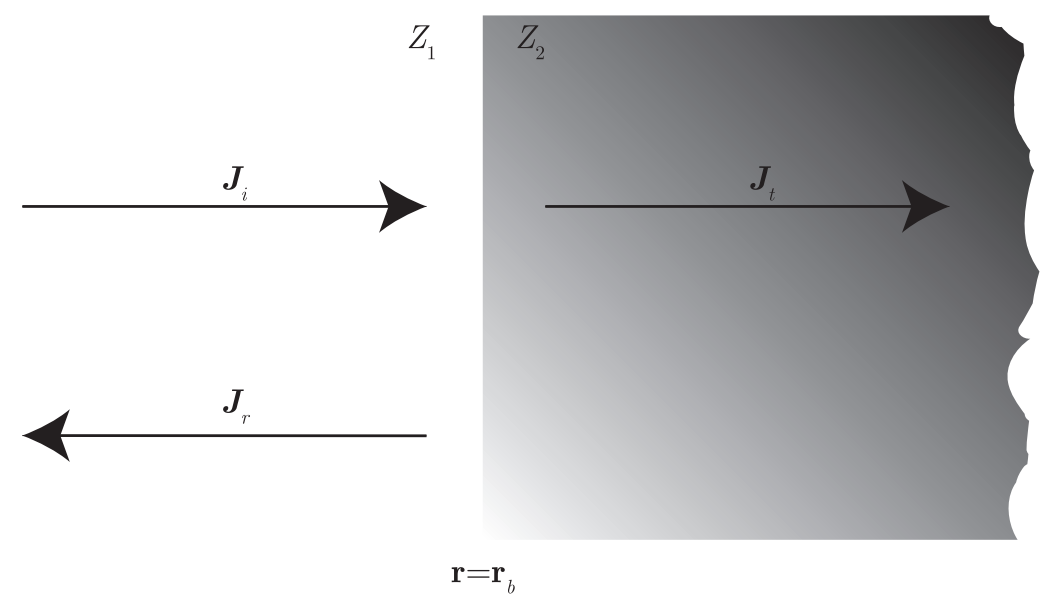

Figure 2.3. Reflection and transmission of acoustic waves.

Reflected energy from an impacting wave can be defined as having two 
possible categories of reflection: specular and diffuse. The following Fig. 2.4 shows how sound energy is reflected in the defined categories. A specular reflection of sound (see Fig. 2.4.a) obeys Snell's law, i.e., the angle of reflection is equal to the angle of incidence, $\cos \theta_{i}=\cos \theta_{r}$, like the mirror reflection of light. A specular reflection can be obtained approximately from a plane, rigid surface with dimensions much larger than the wavelength of the incident sound [ISO, 2004]. Diffuse reflection occurs when the size of the irregularities and the wavelength are similar. In a diffuse reflection the sound incident to a surface is reflected into a wider solid angle than that of a specular reflection [Howarth and Lam, 2000], and it is said that the sound has been scattered [Lam, 1996]. As Fig. 2.4.c shows, an ideal diffuse reflection surface scatters energy according to Lambert's cosine law [Vorländer, 2008]. The intensity of a Lambert scatterer (see Eq. (2.35)) depends on the cosine of the angle of reflection, $\cos \theta_{r}$ and it is independent of the angle of incidence, $\cos \theta_{i}$. Scattering of sound inevitably occurs when sound is reflected from a surface, not only by the fact that real life surfaces are not perfectly smooth nor uniform, but also because of the effects created by their finite size. Fig. 2.4.b shows a general real situation where part of the reflected energy is specularly reflected and the other part diffusely reflected composing a so-called mixed reflection. Moreover, this real reflection behaviour has a strong frequency dependence, i.e. the relation between the size of surface's irregularities or the obstacle and the sound wavelength.

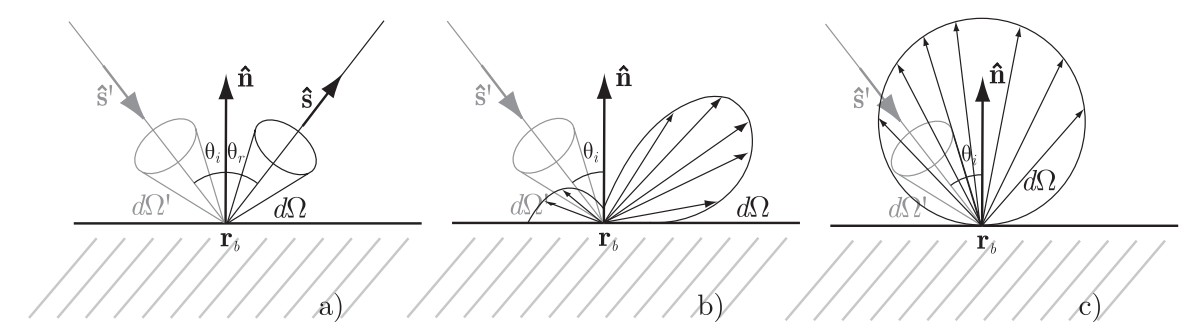

Figure 2.4. Different categories of sound reflection; (a) specular reflection, (b) mixed reflection, and (c)diffuse reflection. 
Following with the scenario that Fig. 2.3 shows, the sound energy of the incident wave is splitted between the reflected and the transmitted waves, assuming that there is no absorption [Everest, 2001],

$$
J_{i}\left(\mathbf{r}_{b}\right) \cos \theta_{i}=J_{r}\left(\mathbf{r}_{b}\right) \cos \theta_{r}+J_{t}\left(\mathbf{r}_{b}\right) \cos \theta_{t} .
$$

where $J\left(r_{b}\right)=\left\|\mathbf{J}\left(\mathbf{r}_{b}\right)\right\|$ and $\mathbf{r}_{b}$ a position at the boundary.

If specular reflection occurs and dividing by $J_{i}\left(\mathbf{r}_{b}\right) \cos \theta_{i}$ yields,

$$
1=\frac{J_{r}\left(\mathbf{r}_{b}\right)}{J_{i}\left(\mathbf{r}_{b}\right)}+\frac{J_{t}\left(\mathbf{r}_{b}\right)}{J_{i}\left(\mathbf{r}_{b}\right)} \frac{\cos \theta_{t}}{\cos \theta_{i}}=R_{F}\left(\mathbf{r}_{b}, \theta_{i}\right)+T_{F}\left(\mathbf{r}_{b}, \theta_{i}\right)
$$

where the first term $R_{F}$ is called the reflection coefficient and the second term $T_{F}$ is the transmission coefficient. The amplitude of $R_{F}$ describes how well the reflecting boundary or surface reflects sound.

For simplicity let us assume that a plane wave in fluid 1 strikes the surface of fluid 2 at normal incidence

$$
\begin{gathered}
R_{F}=\frac{J_{r}(r)}{J_{i}(r)}=\left(\frac{Z_{2}-Z_{1}}{Z_{2}+Z_{1}}\right)^{2}, \\
T_{F}=\frac{J_{t}(r)}{J_{i}(r)}=\left(\frac{4 Z_{1} Z_{2}}{Z_{1}+Z_{2}}\right)^{2},
\end{gathered}
$$

which shows that the wave is almost fully reflected $\left(R_{F} \simeq 1\right)$ if $\rho_{2} c_{2} \gg \rho_{1} c_{1}$, or $\rho_{2} c_{2} \ll \rho_{1} c_{1}$, and not reflected at all if $\rho_{2} c_{2}=\rho_{1} c_{1}$, irrespective of the individual properties of $c_{1}, c_{2}, \rho_{1}$ and $\rho_{2}$.

\subsubsection{Absorption}

There are two mechanisms of sound absorption in room acoustics. The first is a consequence of atmospheric absorption effects [Bass et al., 1972]. The second is a consequence of the absorbent properties of surfaces.

2.2.7.2.1 Sound absorption in the air. A sound wave travelling through the air is attenuated by a factor $m$, which depends on the temperature and the relative humidity of the air [Bass et al., 1972]. The unit of the air attenuation factor is $\mathrm{m}^{-1}$. In general, this attenuation increase if temperature 
and humidity of the air decrease. If this attenuation is included, the sound intensity for a plane wave is

$$
J(r)=J_{0} e^{-m\left(r-r_{0}\right)},
$$

where $J_{0}$ is the sound intensity at $r_{0}$ position and $\left(r-r_{0}\right)$ is the travelled distance.

If the room is small, the distance travelled by sound waves will be small also, so air absorption could be neglected. However, in bigger rooms the atmospheric absorption can have a major impact. Moreover the air attenuation factor depends on the sound frequency. If the ambience conditions are constant, the factor increases with frequency. This effect can be important in big rooms from 3,000 Hz. Some typical values of $m$ are found in Table 2.1 .

\begin{tabular}{lccccccc}
\hline \hline Frequency band $(\mathrm{Hz})$ & 125 & 250 & 500 & 1,000 & 2,000 & 4,000 & 8,000 \\
\hline $\begin{array}{l}\text { Air } \\
\text { absorption }\left(10^{-3} \mathrm{~m}^{-1}\right)\end{array}$ & 0.095 & 0.305 & 0.699 & 1.202 & 2.290 & 6.240 & 21.520 \\
\hline \hline
\end{tabular}

Table 2.1. Air absorption exponents at $23^{\circ} \mathrm{C}, 50 \%$ relative humidity, and normal atmospheric pressure in $10^{-3} \mathrm{~m}^{-1}$.

2.2.7.2.2 Surface absorption. The second mechanism of sound absorption in room acoustics is caused by the absorption characteristics of the surfaces. In this case, absorption is defined as the dissipation of sound energy upon striking a physical surface [Kuttruff, 4th edition, 2000]. At every specular reflection, a portion $\alpha\left(\mathbf{r}_{b}\right)$ of its energy or power is absorbed

$$
J\left(\mathbf{r}_{\mathbf{b}}, \theta_{r}\right)=J\left(\mathbf{r}_{\mathbf{b}}, \theta_{i}\right) R_{F}\left(\mathbf{r}_{b}\right)=J\left(\mathbf{r}_{\mathbf{b}}, \theta_{i}\right)\left(1-\alpha\left(\mathbf{r}_{b}\right)\right)
$$

where $J\left(\mathbf{r}_{\mathbf{b}}, \theta_{i}\right)$ is the incoming sound energy from direction $\theta_{i}$ at $\mathbf{r}_{b}$ boundary position. Otherwise, if an ideal diffuse reflection happens, then the 
energy is scattered in all directions according to Lambert's cosine law such that:

$$
J\left(\mathbf{r}, \theta_{r}\right)=J\left(\mathbf{r}_{\mathbf{b}}\right) R_{F}\left(\mathbf{r}_{b}\right) \cos \theta_{r}=J\left(\mathbf{r}_{\mathbf{b}}\right)\left(1-\alpha\left(\mathbf{r}_{b}\right)\right) \cos \theta_{r} .
$$

This factor $\alpha\left(\mathbf{r}_{b}\right)$ is called the absorption coefficient. The portion of sound energy that is not absorbed is then either bounced back as reflected sound, or transmitted through the material. However, when studying the acoustics of an enclosure, the factor which is transmitted can be considered as being absorbed, thus $R_{F}\left(\mathbf{r}_{b}\right)=\left(1-\alpha\left(\mathbf{r}_{b}\right)\right)$. For a material of infinite thickness and normal incidence, $\alpha$ can be approximated to $T_{F}$ in Eq. (2.32). In real materials with finite thickness and random incidence, a standardized method for measuring the absorption coefficients of materials is used (ISO/DIS 354) [ISO, 2003]. It is important to note that the absorption coefficient is frequency dependent.

2.2.7.2.3 Equivalent absorption area of a room. If the absorption is distributed locally over the walls of a room, the mean absorption coefficient can be estimated by accounting for the surface areas of the materials having different absorption properties [Kuttruff, 4th edition, 2000]

$$
\begin{array}{r}
\bar{\alpha}=\frac{A}{S_{t}}=\frac{1}{S_{t}} \sum_{i=1}^{N} S_{i} \alpha_{i}, \\
S_{t}=\sum_{i=1}^{N} S_{i}, \\
A=\sum_{i=1}^{N} S_{i} \alpha_{i},
\end{array}
$$

where $A$ is the so-called equivalent absorption area of a room that has units of $\mathrm{m}^{2} . S_{t}$ is defined as the total interior surface area, $S_{i}$ is the area of each surface with different absorption coefficient $\alpha_{i}$ and $N$ is the total number of room surfaces with different absorption coefficients. 
To take into account the absorption of objects within the room Eq. (2.38) is modified such that:

$$
A=\sum_{i=1}^{N} S_{i} \alpha_{i}+\sum_{o=1}^{N_{o}} S_{o} \alpha_{o},
$$

with $N_{o}$ being the total number of objects with different absorption coefficient $\alpha_{o}$ and with its respective area $S_{o}$.

The air absorption attenuation described above has to also be included as absorption of the room, which modifies the previous Eq. (2.39) as follows [Kuttruff, 4th edition, 2000]:

$$
A=\sum_{i=1}^{N} S_{i} \alpha_{i}+\sum_{o=1}^{N_{o}} S_{o} \alpha_{o}+4 m V .
$$

\subsection{Sound in an enclosed space}

After the basic physical foundations of the acoustic wave have been presented in previous Sec. 2.2, it is necessary to explain the behaviour of sound in enclosed spaces. With those mathematical models, free field propagation can be studied and simulated. However, numerous problems are related to sound in rooms, whether small as in vehicles, a little larger as in living rooms, or rather large as in concert halls. Up until now, free field conditions have been assumed since an unbounded space had been considered. However, a room or enclosure is defined as a bounded space, where the iterative reflections of the propagated waves in different walls form the total sound field. Then, the total sound field in an enclosure is the result of a set of wave phenomena such as reflection, absorption, diffraction,... particularized on the room properties under analysis.

It is not within the scope of this thesis to deal with a detailed overview of room acoustics (this can be consulted in [Kuttruff, 4th edition, 2000]). However, some basis of the sound propagation in enclosures used throughout this thesis are presented. Firstly, this section presents the temporal sound 
propagation phenomenon, focusing on the absorption and reflection events at boundaries. Secondly, different approaches to describe the sound pressure field in a room are reviewed. Lastly, this section examines how the particular acoustic properties of a room can be evaluated and several room-acoustic parameters can be calculated.

\subsubsection{Sound propagation within rooms}

An enclosed space is defined as an acoustic environment that is bounded by physical objects such as walls, floor, and ceiling. This situation is different from the behaviour of sound outdoors in a free field where reflection events do not occur. A room or hall can be considered as a typical enclosed space. In general terms, every room built to emit and receive sound messages can be considered as a communication system where a given input signal results in a corresponding output [Vorländer, 2008]. As showed in Fig. 2.5, this system is composed of three basic elements: emitter, transmission channel and receiver. The message, or input, is transmitted through the system and tries to get, with the best quality, to the receiver, or the output. As explained in Sec. 2.2, the acoustic message, mainly speech or music, is transported by sound waves that have a specific frequency spectrum. These waves, emitted by the sound source, such as a voice, an instrument or a loudspeaker, are transformed, altered, distorted, and filtered by the enclosed space, or the system, according to its physical properties, such as the dimensions, the number of surfaces, and how absorptive these surfaces are [Beranek, 1954]. Moreover, the sound waves are partially masked by the background noise in the room. Finally, the sound waves reach the receiver, such as a measurement device or the auditory system of a listener, who rates the quality of this room in transmitting this kind of sound message by objective and subjective parameters [Vorländer, 2008].

When a point sound source starts to emit energy in all directions, a spherical wave is created. Sound pressure is inversely proportional to distance, as showed in Eq. (2.16), so the energy decreases with the travelled 


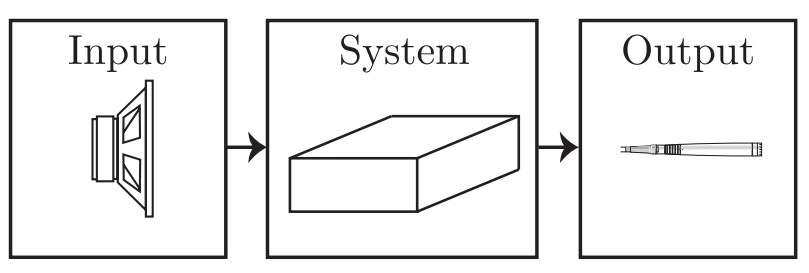

Figure 2.5. Room as a communication system.

distance as in Eq. (2.33). However, if the sound wave impacts on a boundary of the room, the sound wave propagation stops and part of its energy is reflected and the other part is absorbed. As explained in Sec. 2.2.7, these reflections can be defined as having two possible categories of reflection: specular and diffuse (see Fig. 2.4). In real life, both phenomena occur in each reflection, with different proportions, depending on the wall shape.

Therefore, the sound energy at a position of the room at a specific instant is obtained by a superposition of energies from the several waves, both impacted and reflected, arriving at this position and at this specific instant. To examine this sound field, a fixed source, which emits a short impulsive sound, is considered as the input of the system, and a fixed listener, who receives the sound propagated in this room as the output of the system [Vorländer, 2008]. There are two different propagation ways for sound to arrive at the listener's position: the direct way, and the reflected way, and its temporal distribution of the energy can be divided in three stages [Kuttruff, 4th edition, 2000].

An echogram or discrete energy decay response is a graph that shows the sound intensity of the different sound waves that arrive to the receiver as a function of their delay times [Dalenbäck et al., 1992]. Fig. 2.6 shows an example describing the temporal distribution of the energy in these three stages. 


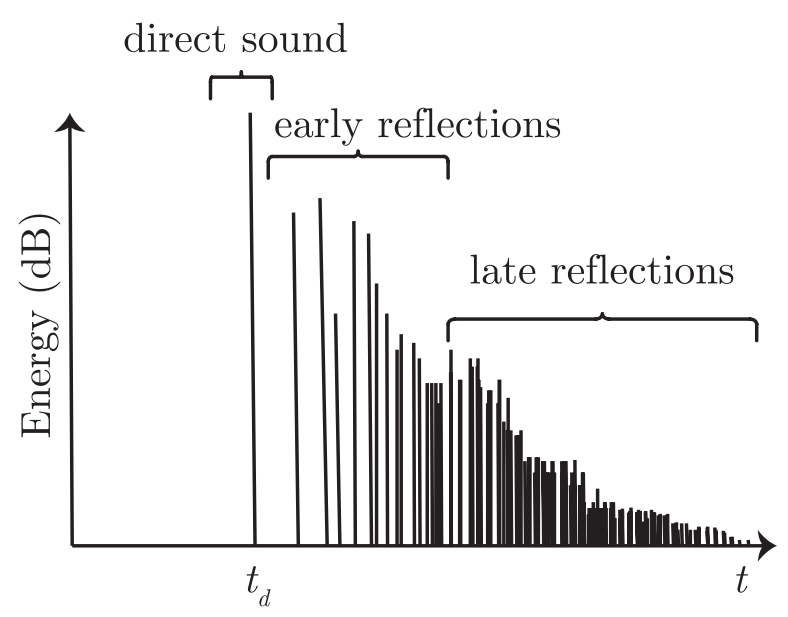

Figure 2.6. Temporal distribution of the energy: Direct sound, early reflections and late reverberation.

\subsubsection{The direct sound}

The first sound that arrives at every listener in a room is the direct sound from the source. This first acoustic energy arrives with a time delay that depends on the distance the sound has travelled, in a straight line, and can be calculated with the definition of the speed of sound in Eq. (2.2). It will occur at time $t_{d}$ after $t=0$ as shown in Fig. 2.7.

Fig. 2.7 indicated that the precise time distribution of these early reflections will vary according to the exact size and shape of the enclosed space.

The direct sound carries the original sound with an attenuation due to spherical divergence and air absorption. It has a slight frequency degradation from the emitted sound, due to the air absorption; that is frequency dependent [Bass et al., 1972]. 


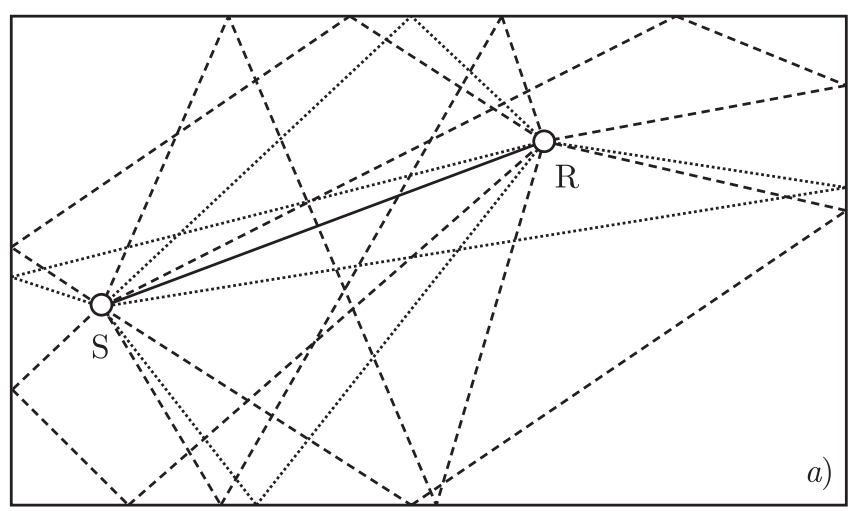

Direct sound First reflections Second order reflections

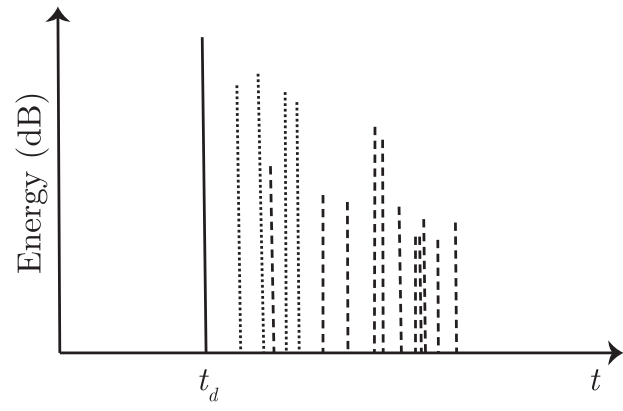

b)

Figure 2.7. (a) Propagation path of direct sound and early reflections.(b) Echogram.

\subsubsection{Early reflections}

Just after the direct sound arrives, a set of reflected sounds from walls and objects within the room arrive at the listener. This set of discrete sounds are called early reflections. Each reflected sound will arrive with a delay time equal to the ratio between the total travelled distance by the sound waves from the source to the receiver, and the speed of sound. They are separated by time, level and direction from the direct sound and each other. 
If these early reflections are separated by more than about $30 \mathrm{~ms}$ from each other then they will be perceived as discrete echoes [Howard and Angus, 1996]. In addition to the attenuation due to spherical divergence and air absorption, as set for direct sound, which are higher because they travel a longer distance, the early reflections are affected by another attenuation. This additional attenuation happens in the reflection events where a portion of sound energy is absorbed, basically depending on the sound frequency and the features of the material that covers the external surface of the room elements. Therefore, these reflected sounds have higher frequency degradation than the direct sound. Moreover, the level, arrival time and direction of the early reflections will vary considerably according to the relative positions of source and listener and the geometry of the room. They give the listener information about the shape and the size of the room and the location of the sound source within it.

\subsubsection{The reverberant sound}

After a large number of early reflections have arrived, sound continues to be received at the listener's position coming from a increasing number of multiple-reflected paths. However, these late reflections are weaker than those that have arrived previously, due to a higher number of reflection events that absorb acoustic energy. This superposition phenomenon of sound waves that are delayed in time and are highly attenuated, coming from the source and successive reflections, and perceived as a continuous and gradual decaying sound of the original impulsive event is called reverberation. The reverberant sound differs from the direct sound and early reflections in that it is diffuse. It means that all directions of sound propagation provide the same sound intensity and so the distribution of the sound energy is homogeneous and isotropic at each instant and position of the room.

If the sound source is continuous, it will build up until it reaches an equilibrium level where the whole room absorbs the same energy by unit time as the sound source emits. In this steady-state situation the mean 
energy density around the room remains constant. This only occurs after enough reflection events have happened and the early reflection stage has ended, Therefore, this is dependent on the grade of diffuse reflection and absorption in the room as well as its size. When the source stops emitting, sound density energy accumulated in the room does not immediately disappear, but the sound level decreases at a rate determined by the size of the space and the amount of sound energy absorbed in each reflection.

\subsubsection{The room impulse response}

The introduction of Sec. 2.3.1 presented how an acoustic enclosed space can be considered as a communication system. In general, an acoustic room is a linear time invariant (LTI) system where the output signal can be obtained by performing the convolution of the input signal with the system's impulse response [Oppenheim et al., 1996]. Fig. 2.8 shows a diagram of an LTI system where $x(t)$ is the input signal, $y(t)$ is the output signal and $h(t)$ is the system's impulse response.

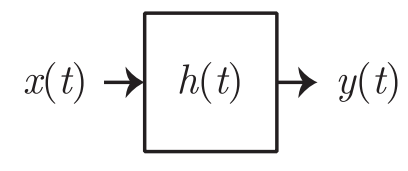

Figure 2.8. Room as a linear time invariant system

A system is linear if it satisfies both homogeneity and superposition conditions. Consider a system with input $x(t)$ and output $y(t)$,

$$
x(t) \rightarrow y(t) .
$$

- Homogeneity. If the input $x(t)$ in Eq. (2.41) is scaled by a factor $k$, the output is scaled by the same factor,

$$
x_{1}(t)=k x(t) \rightarrow y_{1}(t)=k y(t) .
$$

- Superposition. If a system is given two input simultaneously, the output is the sum of individual outputs caused by the application of 
each inputs separately. Let

$$
\begin{aligned}
x_{1}(t) & \rightarrow y_{1}(t), \\
x_{2}(t) & \rightarrow y_{2}(t) .
\end{aligned}
$$

If

$$
x(t)=x_{1}(t)+x_{2}(t),
$$

then

$$
y(t)=y_{1}(t)+y_{2}(t)
$$

By combining the two properties, the linearity is expressed in the following manner, if

$$
x(t)=k_{1} x_{1}(t)+k_{2} x_{2}(t)
$$

then

$$
y(t)=k_{1} y_{1}(t)+k_{2} y_{2}(t) .
$$

A linear time invariant system fed with the unit impulse function, or the Dirac delta function $\delta(t)$ will yield an output signal called the system's impulse response $h(t)$ [Oppenheim et al., 1996]. $\delta(t)$ is an infinite amplitude signal, with non time duration and unity area. The delta function is graphed as Fig. 2.9.a shows. In $t=0$ its amplitude has an infinite value, and the arrow's height represents its area. Normally a scaled impulse $k \delta(t)$ is drawn as Fig. 2.9.b.

Convolution integral is an operation that allows the output signal of any linear time invariant system to be found in response to any given input signal using the impulse response [Oppenheim et al., 1996],

$$
y(t)=\int_{-\infty}^{+\infty} x(\tau) h(t-\tau) d \tau=x(t) * h(t)
$$



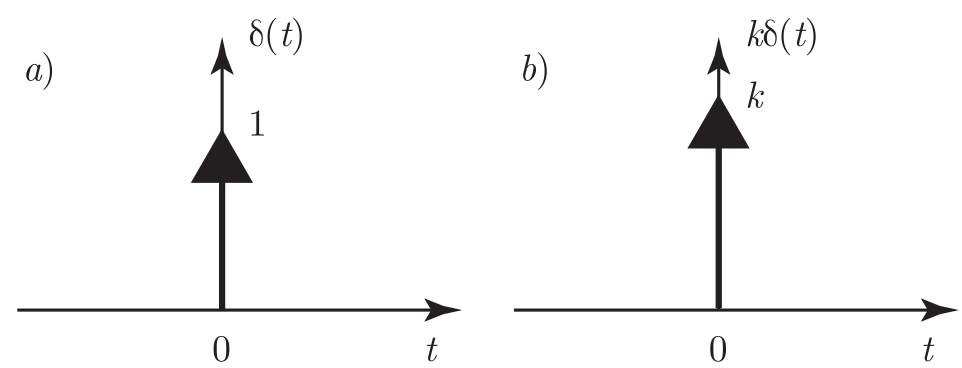

Figure 2.9. (a) unit impulse function (b) scaled unit impulse function.

where $*$ is the convolution operator.

By analogy, the Room Impulse Response (RIR) can define the acoustic properties of an enclosed space. One unique RIR for each pair of sourcelistener position in a room exists. The RIR of a real room can be measured by different methods. The most commonly used RIR measurement techniques are Time Delay Spectrometry (TDS) [Heyser, 1967] and Maximum Length Sequence Signal Analysis (MLSSA) [Rife and Vanderkooy, 1989]. In this thesis, the focus is pointed on computer modelling of room acoustics that tries to estimate this RIR in order to calculate the room acoustic criteria presented in Sec. 2.3.3. Generally, these are derived from the measured or calculated RIR using the following approximations

$$
\begin{gathered}
p(t) \approx h(t), \\
w(t) \approx h^{2}(t) .
\end{gathered}
$$

Auralisation [Vorländer, 2008] has been included in room acoustic simulation by convolution of the RIR with sound recordings from an anechoic chamber. This chamber is a room without any reflecting surfaces that results in the free field conditions. 


\subsubsection{Room acoustics approaches}

In the previous Sec. 2.3.1, the fact that the sound field in rooms is made of a superposition of sound waves has been explained. This sound field behaviour in rooms can be shown by different theories depending in the approximation used to describe the sound wave propagation phenomenon. Geometrical acoustics, statistical acoustics, and wave acoustics are briefly presented in this subsection as an introduction to the theories of room acoustics that are mostly used in modelling techniques as reviewed in Chap. 3.

\subsubsection{Quasi-plane wave approach}

A point sound source radiating in a room generates an acoustic field composed by a superposition of spherical waves [Beranek, 1954]. In Sec. 2.2.4, it has been concluded that when the wavefront is far away from the source, a spherical wavefront can be approximated to a plane wave. This generally happens in big dimension rooms. In these rooms, the sound propagation's direction can be described with the normal vector of the plane wavefront. In this plane wave approach, this direction of propagation is called acoustic ray by analogy with the optic ray. The part of acoustics that studies the sound field distribution through ray tracing is called Geometrical acoustics and its application is based on analogies from optics.

A geometrical study by ray tracing is essential in every room acoustics design. It allows us to model an appropriate room shape or geometry, to analyse the possibility of creating echoes, to predict sound focalization zones, etc. Presently, there is a large number of room acoustics modelling techniques based on this approach as revised in Sec. 3.4.

2.3.2.1.1 Quasi-plane wavefront propagation. In geometrical acoustics, rays are used to describe the sound propagation. A sound ray is a thin line of energy transport. The concept of rays implies that the wavelength and the phase of the sound are neglected, i.e., they are incoherent and can not interference with wave effects, and only the direction of sound en- 
ergy propagation is treated in geometrical acoustics. The ray representing a quasi-plane wave, with an infinite small opening angle $d \Omega$ as Fig. 2.10 shows, may start in any direction and it is assumed that the decay of energy in the ray is representative of the decay of energy in the room [Everest, 2001]. From the basic distance law, it follows that the intensity of the ray decreases with $1 / r^{2}$,

$$
J(r)=\frac{W}{4 \pi r^{2}},
$$

where $W$ is the power of the sound source.

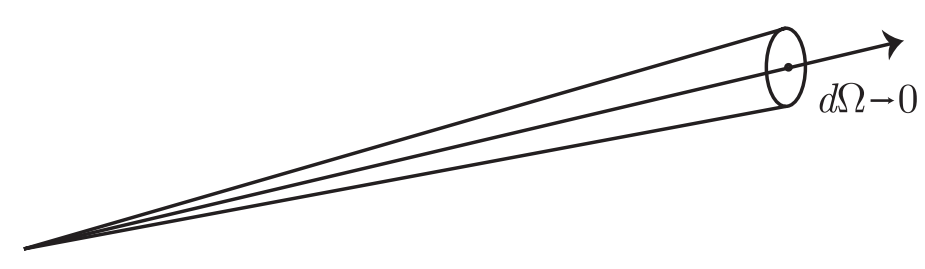

Figure 2.10. Definition of ray.

The room may have any shape. It is supposed that a finite number of acoustic rays emerge from the sound source, which follow the direction of propagation of the sound waves. It has to be noted that these rays have not any real physical meaning [Kuttruff, 4th edition, 2000]. They are just perpendicular lines to the wavefronts. The superposition of two rays is calculated by adding the sound intensities, or the energy density.

\subsubsection{Statistical approach}

If the sound source remains on, the sound continues its propagation in the room impacting, on the boundaries. The number of rays and their solid angles increase with each reflection event covering the whole room. From this moment on the application of the geometrical model is not the most adequate. To study this condition, it is not useful to follow the propagation of each individual ray. Therefore, it is necessary to statistically evaluate the acoustic energy as a whole [Beranek, 1954]. The study of these theories is grouped in Statistical acoustics, that is based in similar foundations as 
the kinetic theory of gases, which states that every sound particle has the same size and energy. Moreover, they are incoherent between them, as the acoustic rays, so the interference effect does not exist and the sound energy can be added without taking into account their phase differences. In the following sections, how the statistical acoustics allows us to model the reverberant sound, to estimate the reverberation time and to predict the sound pressure level of a stationary sound source in a diffuse sound field is presented .

2.3.2.2.1 The diffuse sound field. The basic assumption of statistical reverberation theory is the concept of diffuse sound field. A sound field is expected to be diffuse when sound waves travel in all directions with the same probability and intensity, not only in the steady state but also at any instant of the energy decay stage. Accordingly, the sound energy distribution is homogeneous and isotropic at any instant and location. In such a situation it is possible to speak about random sound incidence.

As enunciated in a previous Sec. 2.3.1.3, reverberation and diffusion are directly related. Moreover, the more diffuse reflection events occur, instead of specular reflection, the more diffusion is obtained [Kuttruff, 4th edition, 2000]. Technical literature [Miles, 1984, Kuttruff, 1995, Hodgson, 1996a] have shown that the concept of a diffuse sound field is a good approximation for proportionate room dimensions and homogeneous distribution of absorption on the room boundaries, and increasing the diffuse reflections versus specular reflections improves accuracy. In special cases, e.g. in corridors or very large room with concentrated absorption, diffuse sound fields will no be present.

On the grounds of the previous subsections it can now be confirmed, that in room acoustics the term diffusion denotes two conceptually different things. Firstly, diffusion is a property of a sound field. It describes an isotropy of directional uniformity of sound propagation. Secondly, diffusion is the ability of a surface to scatter incident sound into non-specular di- 
rections. Although sound field diffusion may be a consequence of diffusely reflecting boundaries, both items must be well distinguished.

2.3.2.2.2 Increase and decrease of the sound energy in a room. The evolution of the sound energy density in a room with time can be divided in three stages as Fig. 2.11 shows.

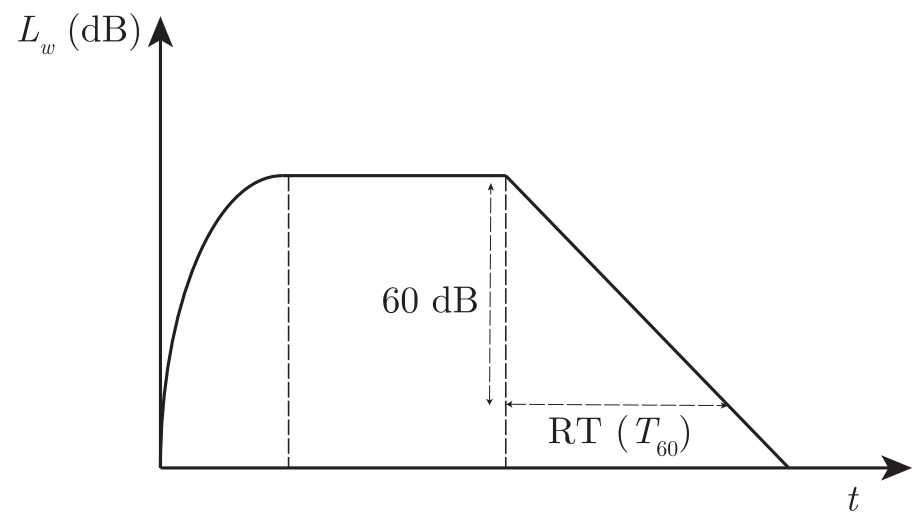

Figure 2.11. Sound energy density level increase, steadystate and decrease in a room. Reverberation time definition.

Firstly, when a sound source starts to emit acoustic energy in an enclosed space, the resulting sound field begins to propagate as in a free field condition. After a certain time that depends on the distance between the walls and the source, the sound waves begin to reflect on the walls and a superposition of impacting and reflecting waves occur. If the source continues emitting energy, these successive reflection events could induce an infinite increase of this energy. However, some energy is absorbed by the room surfaces, by the air and objects within the room. This first stage corresponds to an increasing transitional state of sound energy density with time that is exponential

$$
w(t)=\frac{4 W}{A c}\left(1-e^{\frac{-A c}{4 V} t}\right) .
$$

Then, at a certain instant of time the whole room absorbs the same 
energy for unit time than that emitted by the source and the energy in the room remains stationary around a certain sound level. From this moment the mean energy density in the room remains constant. It can be observed in Eq. (2.52) that the establishment time of this steady state is theoretically infinite obtaining an energy density of

$$
w(t)=\frac{4 W}{A c} .
$$

Finally, if the sound source is suddenly switched off, the accumulated sound energy density will no disappear instantly. But, it decays during a certain period of time because the energy is being absorbed by the room until it is inaudible. Now, the sound energy density decays from the steady state level and its variation only depends on the absorption and the volume of the room

$$
w(t)=\frac{4 W}{A c}\left(e^{\frac{-A c}{4 V} t}\right) .
$$

This phenomenon is of importance for checking the adequacy of the room for performance of speech or music. The sound persistence in a room after the source stops emitting is called reverberation. Sometimes it can be beneficial, as a reinforcement and extension of musical sounds, and sometimes, however, prejudicial, when masking successive vocal sounds in speech [Barron, 2000].

2.3.2.2.3 Reverberation time, mean free path and critical distance. In the analysis of a room for audition qualities, it is important to establish some indexes which allow us to evaluate the acoustic quality of the room as a whole. Moreover, acousticians need a quantifiable objective parameter to characterize the overall impression of how sounds are perceived in the room. W. C. Sabine showed that reverberation is an adequate parameter for the evaluation of the acoustic quality of a room by defining the reverberation time (RT) ${ }^{2}$ [Sabine, 1964] in seconds as the required time for a level decrease of $60 \mathrm{~dB}$ from the stationary level when the sound source is switched

\footnotetext{
${ }^{2}$ It is also abbreviated with $\mathrm{T}_{60}$.
} 
off. As Fig. 2.11 graphs, it represents the decay rate of the sound or the velocity of this process, not the duration of the reverberation effect that it is theoretically infinite. The famous Sabine's equation is

$$
\mathrm{RT}=0.16 \frac{\mathrm{V}}{\mathrm{A}} .
$$

This formula has been shown to be valid for low absorption. When the mean absorption coefficient is over 0.2 , this equation has error values of over $10 \%$ from the measured value [Hodgson, 1996a]. For room with high absorption, the assumption that the energy decays proportionally to the overall energy remaining in the room is wrong because the energy lost by absorption in the reflections is truly discontinuous. The diffuse sound field is otherwise created by numerous reflections of sound waves or particles hitting walls. Using statistical terms, the mean free path between two consecutive reflections is dependent on the ratio of the volume $V$ to the surface area [Pierce, 1994, Kosten, 1960]

$$
\lambda=\frac{4 V}{S_{t}} .
$$

With this assumption, Eyring's reverberation time is enunciated as [Eyring, 1930]

$$
\mathrm{RT}=0.16 \frac{V}{-S_{t} \ln (1-\bar{\alpha})} .
$$

The air absorption attenuation introduced in Sec. 2.2.7.2.1 has to be included in the reverberation time formulae. The more reverberant the room is, the greater the relative influence of the air absorption [Kuttruff, 4th edition, 2000]. According to Eq. (2.33), the energy loss is given by $e^{-m c t}$ which modifies the result of Sabine's and Eyring's reverberation formulae using Eq. (2.40).

Using the statistical theory, the total sound energy density in a room can be predicted as composing the direct field, as in free field conditions Eq. (2.26), with the reverberant field, as in the steady state Eq. (2.53)

$$
p_{\mathrm{rms}}^{2}(\mathbf{r})=W \rho_{0} c\left[\frac{1}{4 \pi r^{2}}+\frac{4}{A}\right] .
$$




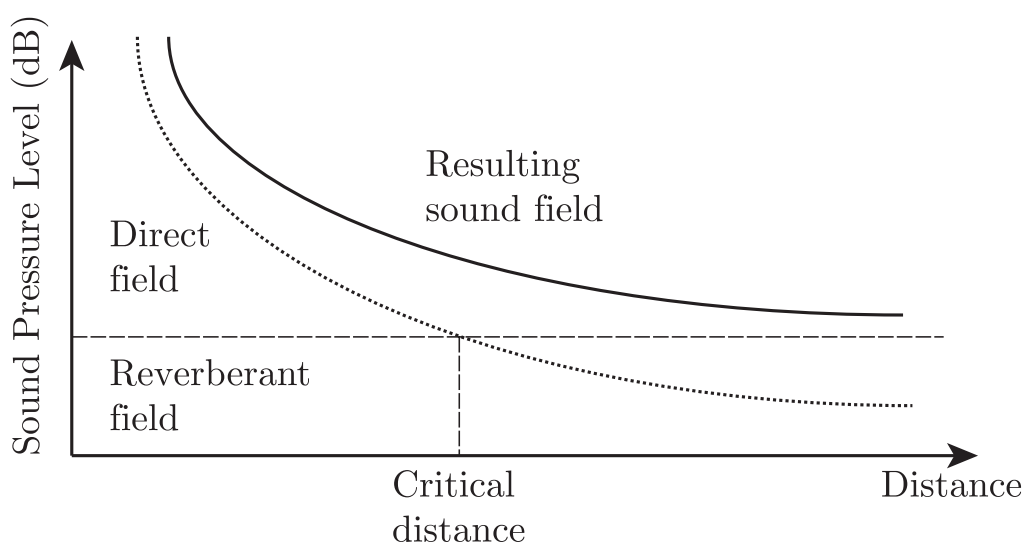

Figure 2.12. Evolution of direct, reverberant and the resulting sound field with distance. Definition of critical distance.

Then, the sound pressure level can be obtained by

$$
L_{p}(\mathbf{r})=L_{W}+10 \log \left[\frac{1}{4 \pi r^{2}}+\frac{4}{A}\right] .
$$

The distance of equal contribution of each field is the so-called critical distance

$$
r_{c}=\frac{1}{4} \sqrt{\frac{A}{\pi}}
$$

Fig. 2.12 graphs the relation between each field and the distance from the source where the critical distance is highlighted.

\subsubsection{Wave equation approach}

Two previous acoustics theories do not take into account the wavelike real physical behaviour of sound as the wave acoustics does. It is based on the resolution of the sound wave differential equation in each room, Eq. (2.3), together with its specific boundary conditions. This settlement shows that each space region of a room represents a system with the ability to vibrate in its three dimensions. In complex rooms, solving this boundary problem can be extremely complicated, even with computer help. Therefore, this method is mainly applied as a theoretical basis to solve specific problems which 
are only understandable by the wave nature of sound. Several modelling techniques have been developed based on this approach. Mainly, they obtain numerical solutions of the wave equation for rooms with complex shape. These techniques are revised in Sec. 3.3. As an useful approach, the room modes in a rectangular room are presented in the following paragraphs.

2.3.2.3.1 Room modes. If an object has the ability to vibrate, it always vibrates in particular ways which correspond to what it is called natural vibration modes [Morse and Ingard, 1968]. Then, if a diapason is hit, it will always vibrate with the same frequency creating an almost pure sound wave. In the same way, when a guitar's tightly stretched string is played, it always vibrates with certain modes or with a combination of them which produces a musical note. The three first modes of a taut guitar string can be observed in Fig. 2.13. The characteristics of these modes depend on the geometry, the material of the object and the boundary conditions.
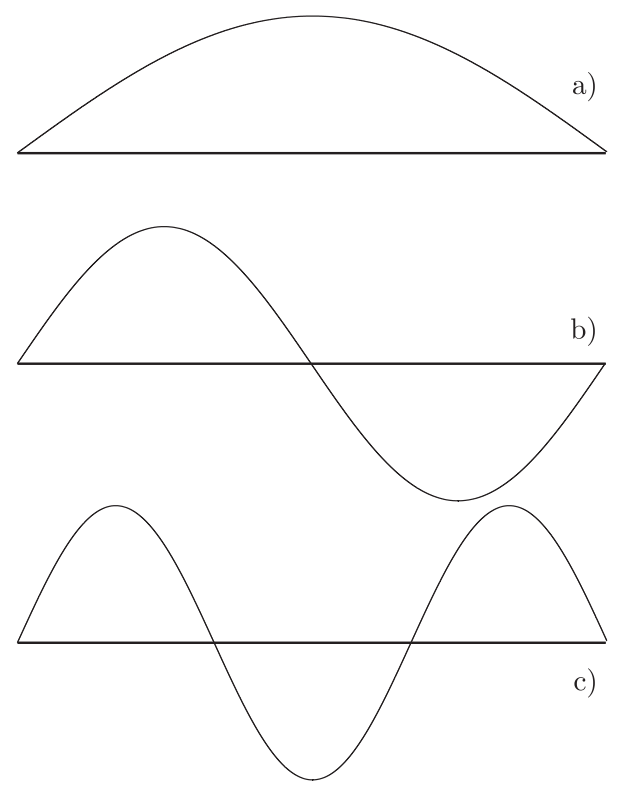

Figure 2.13. Three first vibrating modes of a string. 
Analogously, an enclosed space where the sound is propagated has the ability to vibrate. These room vibration modes or room modes are related with specific frequencies called natural frequencies explained by standing waves creation within the room. A standing wave is a non-progressive wave that after travelling across the room arrives to its start position. The energy does not propagate out of the path of this wave. This phenomenon results in an extremely heterogeneous distribution of the sound pressure in the path of this standing wave with positions with minimum sound pressure or zero (nodes) and other with maximum sound pressure (antinodes) at all time instants appearing [Howard and Angus, 1996]. An example of the nodes and antinodes caused by a standing wave between two rigid parallel walls is shown in Fig. 2.14.

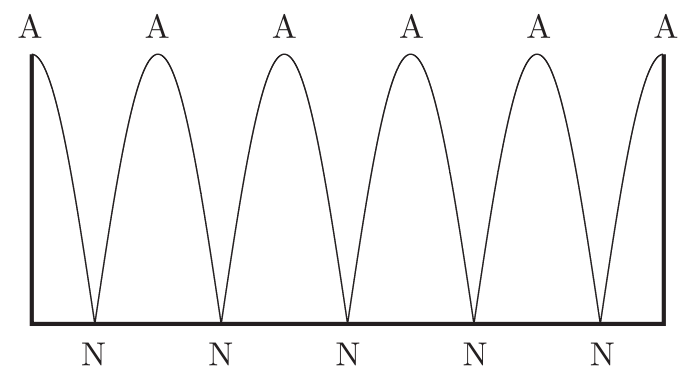

Figure 2.14. Nodes and antinodes of a standing wave between two rigid parallel walls.

If a sound source within a room emits sound waves whose frequencies match those found in the natural frequencies of the room modes, the resonance of sound energy at these frequencies results. The resonance phenomenon is due to the excitation of room modes that create standing waves in different directions and frequencies. The other frequencies emitted by the source, that do not meet the resonance conditions, disappear after a certain number of reflections. However, the excited modes will increase their vibration amplitude and its duration will be longer due to the complementary energy that has been provided by the room. If the listener position 
is situated at an antinode, the amplitude of the room mode will be reinforced in comparison with other received frequencies. On the contrary, the room mode will not be heard at a node position when compared to other frequencies.

These interferences' network and the propagation of standing waves cause an extremely heterogeneous sound field with consequent loss of acoustic quality in the room.

2.3.2.3.2 Standing waves in a rectangular room. Natural frequencies of a room depend on its geometry, dimensions and boundary properties. In a rectangular room that has the dimensions $l_{x}, l_{y}$, and $l_{z}$ in meters in the perpendicular $x, y$ and $z$ axes of the cartesian coordinates system as shown in Fig. 2.15, the wave equation Eq. (2.3) can then be written

$$
\frac{\partial^{2} p(\mathbf{r}, t)}{\partial x^{2}}+\frac{\partial^{2} p(\mathbf{r}, t)}{\partial y^{2}}+\frac{\partial^{2} p(\mathbf{r}, t)}{\partial z^{2}}-\frac{1}{c^{2}} \frac{\partial^{2} p(\mathbf{r}, t)}{\partial t^{2}}=s(\mathbf{r}, t) .
$$

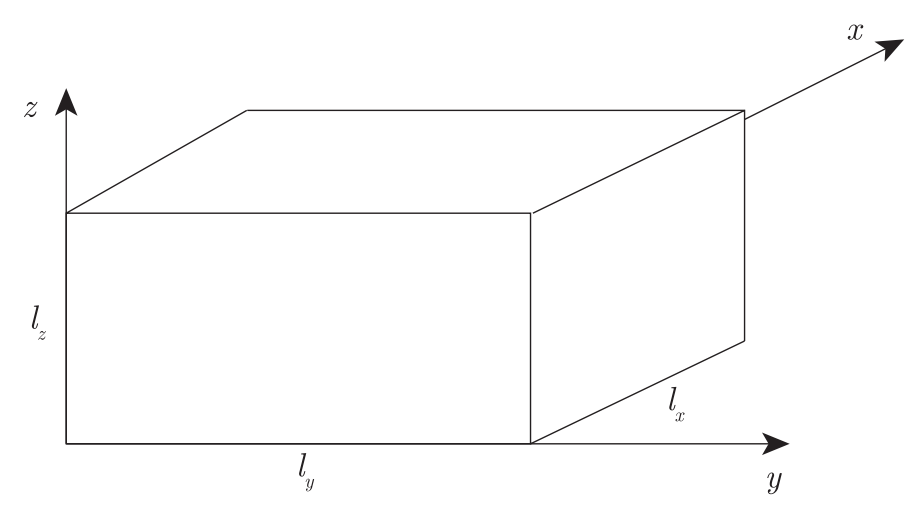

Figure 2.15. Rectangular room with the cartesian coordinates.

One of the solutions of the wave equation leads to a straightforward method of calculating the room modes that are present in a rectangular enclosure [Rayleigh and Lindsay, 1945]. In this case, the room surfaces are 
now assumed to be rigid, i.e. the normal component of the particle velocity is zero at the boundaries. It is possible to calculate the natural frequencies related with the room modes using the following equation

$$
f_{n}=\frac{c}{2} \sqrt{\left(\frac{n_{x}}{l_{x}}\right)^{2}+\left(\frac{n_{y}}{l_{y}}\right)^{2}+\left(\frac{n_{z}}{l_{z}}\right)^{2}},
$$

where $n_{x}, n_{y}$ and $n_{z}$ are positive integer numbers, $0,1,2, \ldots$ Each combination of three so-called directional cosines $\left(n_{x}, n_{y}, n_{z}\right)$ obtains a room mode. There are three different groups of modes:

- Axial modes are one-dimensional and have a direction of propagation parallel to the axis. Two directional cosine of the combination must be zero. The standing wave travels between two parallel planes. Mode $(1,0,0)$ would correspond to the first axial mode along the length.

- Tangential modes are two-dimensional and only one of the directional cosines is zero. These modes are caused when the direction of propagation is parallel to one plane. Mode $(1,1,0)$ would correspond to the first tangential mode between the four walls.

- Oblique modes are three-dimensional, all three directional cosines are non-zero. The standing waves do not travel parallel to any wall.

In room acoustics, it is well-known that the density of modes increases with frequency, independent of the room shape [Kuttruff, 4th edition, 2000]

$$
\frac{d N_{f}}{d f} \approx 4 \pi V \frac{f^{2}}{c^{3}}
$$

where $N_{f}$ denotes the number of nodes.

The number of modes will increase with the frequency used so that at low frequencies less standing waves are created than at high frequencies. Therefore, at high frequencies the sound field heterogeneities will soften because there are multiple room modes. Moreover, the density of room modes 
per hertz increases with room volume. Clearly the natural frequencies of a room can cause acoustic problems, mainly in small room at low frequencies.

It has been concluded in technical literature [Schroeder and Kuttruff, 1962] that, above a certain critical frequency related with volume and reverberation time (see Sec. 2.3.1.3), the resonances are heavily overlapped and the sound field appears homogeneous and diffuse. The frequency range where this overlap is given, starts above

$$
f_{\text {limit }} \approx 2,000 \sqrt{\frac{\mathrm{RT}}{V}},
$$

where $f_{\text {limit }}$ is called the Schroeder's frequency. Below it, the modes are more spaced between them and can be perceived by the listener.

\subsubsection{Room-acoustic quality parameters}

Although the psychoacoustic evaluation of the sound by the listener within the room plays an important role in its rating and design, these subjective criteria depend on the audience and acoustics performers knowledge and training, so these criteria could be unclear and non comparable between them. Subjective studies of the acoustical characteristics of auditoria have shown that several quantities that can be obtained from the RIR (see Sec. 2.3.1.4) are correlated with particular subjective aspects of the character of a room. While reverberation time, presented in Sec. 2.3.2.2.3, is one of the fundamental description of the acoustical character of an auditorium, the addition of more quantities gives a more complete description of the acoustical conditions in a room. Therefore, the definition of objective parameters, which can be measured or calculated and being directly related with the subjective sensations of the listener, are desirable and also important acoustic design considerations. In this section, some fundamental quality parameters for room acoustics evaluation are presented. Most of them have been defined by various authors such as Beranek [Beranek, 1962], Ando [Ando, 1985] as well as the ISO 3382 [ISO, 2009]. These quantities will be used in the following chapters to evaluate the acoustic diffusion 
equation model as a room acoustics simulation technique.

The room-acoustical quality criteria can be subdivided into time and energy criteria. The first group includes quantities such as reverberation time and early decay time which are related to the sensation of reverberance. Quantities in energy criteria group such as how early to late sound index relate to perceived definition, clarity or the balance between clarity and reverberance, as well as to the intelligibility of speech. The main use of the room, speech or music, then determines the recommendations for the guide values to be targeted.

\subsubsection{Reverberation time, RT}

The reverberation time is the most important and well known characteristic of an auditorium. It has been defined in Sec. 2.3.2.2.3 as the time that would be required for sound pressure level to decrease by $60 \mathrm{~dB}$ after a stationary sound source is turned off. It is possible to estimate a unique value for all positions of the room using the theoretical formulae of statistical acoustics revised in Sec. 2.3.2.2.3. However, it is also possible to calculate RT from the slope of the integrated room impulse response curves [Schroeder, 1965] (see Sec. 2.3.1.4 for details about RIR). This process consists of two steps; the integration of the energetic impulse response, $p(\mathbf{r}, t)^{2}$,

$$
C(\mathbf{r})=h^{2}(\mathbf{r}, t<0)=\int_{0}^{\infty} p^{2}(\mathbf{r}, \tau) d \tau
$$

and a subtraction of the energy components contained in the impulse response according to their delay,

$h^{2}(\mathbf{r})=C(\mathbf{r})-\int_{0}^{t} p^{2}(\mathbf{r}, \tau) d \tau=\int_{0}^{\infty} p^{2}(\mathbf{r}, \tau) d \tau-\int_{0}^{t} p^{2}(\mathbf{r}, \tau) d \tau=\int_{t}^{\infty} p^{2}(\mathbf{r}, \tau) d \tau$.

The reverberation time can now be evaluated from the decay curve, $h^{2}(\mathbf{r})$. The slope of the decay curve should be determined from the slope of the best fit linear regression line to the appropriate portion of the decay curve. Due to the dependence on the excitation level as well as on the noise level, it is often difficult to achieve the required evaluation dynamic range, especially 
in the low frequency range. The decay rate is normally obtained from 5 $\mathrm{dB}$ and $35 \mathrm{~dB}$ below the maximum initial level and is referred to as $\mathrm{T}_{30}$. When a smaller dynamic range exists, a portion of the decay curve between $5 \mathrm{~dB}$ and $25 \mathrm{~dB}$ can be used, being called $\mathrm{T}_{20}$. Finally the decay time RT is calculated from these decay rates as the time required for $60 \mathrm{~dB}$ to decay.

The reverberation time is used as a single indicator for the characterization of the room as the mean value between the four octave mid bands 250, 500, 1,000 and 2,000 Hz. It is called the mean reverberation time.

\subsubsection{Early decay time, EDT}

The early decay time is also a time criteria related with the sensation of reverberance and is defined as the time, in seconds, taken for the reverberant sound to decay $10 \mathrm{~dB}$ [Atal et al., 1965]. In this case, the EDT should be obtained from the initial $10 \mathrm{~dB}$ of the decay from the same slope as the RT. It is mostly more in conformity with the subjective assessment of the duration of the reverberation than the RT. This also explains the fact that reverberance subjectively perceived in the room may vary in each location, while the values measured objectively with a high dynamic range $(60 \mathrm{~dB}$ or $30 \mathrm{~dB})$ are generally independent of the location.

\subsubsection{Clarity, $\mathrm{C}_{80}$}

Clarity is an energy criteria that calculates a balance between early and late arriving energy. This quantity allows us to evaluate the time distribution of the reverberant energy, in particular the significance of the direct sound and early reflections compared with the reflections of the diffuse sound field [Reichardt et al., 1974]. This can be calculated for either a $50 \mathrm{~ms}$ or a 80 ms early limit depending on whether or not the results are intended to relate these conditions to speech or music respectively.

$\mathrm{C}_{80}$ is a measure of the ratio of the energy contained in the first $80 \mathrm{~ms}$ of sound compared with the energy from $80 \mathrm{~ms}$ onwards until extinction and is expressed in decibels [ISO, 2009]. 


$$
\mathrm{C}_{80}=10 \log \left(\int_{0}^{80 \mathrm{~ms}} p^{2}(t) d t / \int_{80 \mathrm{~ms}}^{\infty} p^{2}(t) d t\right),
$$

where $p(t)$ is the measured or predicted RIR. It describes the temporal transparency of musical performances.

\subsubsection{Definition, $\mathrm{D}_{50}$}

It is also possible to measure an early to total sound energy ratio. The most known is Definition, $\mathrm{D}_{50}$, which is normally used for speech conditions [ISO, 2009],

$$
\mathrm{D}_{50}=\int_{0}^{50 \mathrm{~ms}} p^{2}(t) d t / \int_{0}^{\infty} p^{2}(t) d t
$$

which is expressed as a percentage.

Definition is related to $\mathrm{C}_{50}$ by the following equation [ISO, 2009]

$$
\mathrm{C}_{50}=10 \log \left(\frac{\mathrm{D}_{50}}{1-\mathrm{D}_{50}}\right)
$$

\subsubsection{Centre time, TS}

For music and speech performance, the centre time TS is a reference value for spatial impression and clarity. It is measured in seconds and corresponds to the instant of the first moment or centre of gravity in the squared impulse response. It is defined in the following equation [ISO, 2009].

$$
\mathrm{TS}=\int_{0}^{\infty} t p^{2}(t) d t / \int_{0}^{\infty} p^{2}(t) d t
$$

TS avoids the discrete division of the impulse response into early and late periods.

\subsubsection{Just noticeable differences, jnd}

In psychoacoustics, a just noticeable difference, normally abbreviated with lowercase letters as jnd, is the smallest detectable difference between a start- 
ing and secondary level of a particular sensory stimulus, that is, the minimal perceptible change in each parameter. It is also known as the difference limen or the differential threshold. According to ISO/DIS 3382, the subjective threshold for the room-acoustic quality parameters presented in this section are shown in Table 2.2.

\begin{tabular}{lccccc}
\hline \hline Parameter & RT & EDT & $\mathrm{C}_{80}$ & $\mathrm{D}_{50}$ & TS \\
\hline jnd & $5 \%$ & $5 \%$ & $1 \mathrm{~dB}$ & $5 \%$ & $10 \mathrm{~ms}$ \\
\hline \hline
\end{tabular}

Table 2.2. Just noticeable difference of several room-acoustic quality parameters according to ISO/DIS 3382.

\subsection{Summary}

In this chapter, some of the fundamentals of the physics involved in the sound wave propagation are introduced. The particular distribution of the sound quantities such as the pressure and particle velocity components, determines the perception of the acoustical properties. It could be possible to mathematically determine those sound field properties through a sound wave equation. Simple solutions of this wave equation facilitate the description of the sound propagation in free field to explain its main properties and effects.

The sound propagation within a room is defined as the consequence of a sound field in a bounded area. The boundary conditions in room acoustics are determined by the absorbing and reflecting properties of the walls. Both specular and diffuse reflections occurs when the impedance of the medium change depending on the size of the irregularities and the wavelength.

The acoustics of an enclosed space can be considered as a generic system with an input and an output that is processed in some manner according to the properties of the space itself. Using a discrete unit impulse radiated by a point source, three different stages in the sound propagation phenomenon 
have been identified; the direct sound, the early reflections and the reverberant sound which determine the acoustic characteristic features of the room and define the so-called room impulse response (RIR).

As a result, the sound field in a room is composed of a superposition of sound waves that come from different directions, and have different amplitude and phase in each one. The sound field behaviour in a room can be shown by different theories depending in the approximation used to describe the sound wave propagation phenomenon. These approaches are useful in the modelling techniques reviewed in Chap. 3 that will be used to predict the RIR.

Finally, the RIR can be used directly to compute a processing operation of auralisation. It can also be used as an analysis tool from which the roomacoustic quality parameters can be obtained. These objective acoustical parameters not only evaluate and rate the room but also correlate to some perceptually measures of acoustic performance. 


\section{Room Acoustics Modelling Techniques}

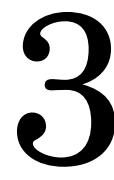

The PREVIOUs CHAPTER introduced some of the physical concepts of the propagation of sound in rooms. As it has been mentioned, there are different ways to model the acoustics of a room, with particular emphasis on the reverberant sound. Except for some simple cases that can be analytically solved, most of the room acoustics modelling techniques are based on approximations of the sound field through several physical simplifications [Schroeder, 1973]. According to the applied approximation theory, several room acoustics simulation methods are derived with their relative merits and disadvantages.

\subsection{Introduction}

The purpose of this chapter is to introduce and revise the main techniques currently applied to room acoustics modelling. 
The physical phenomena involved in sound wave propagation within enclosures are both numerous and complex, making overall analytical modelling difficult. Due to the large number of variables to be taken into account in the description of a real situation, only an approximation of it is possible [Ondet and Barbry., 1989]. As it has been introduced in the previous chapter (Chap. 2), there are three different main approaches; statistical methods, wave-based methods, and geometrical methods [Savioja, 1999].

Computers have been used for over forty years to model room acoustics, and presently computational modelling has turned to be a common practice in the design of buildings with specific acoustic requirements such as concert halls. In this chapter, a revision of these three major approaches is presented with particular emphasis on the most important implementations of each one. A diagram of the revised approaches in this chapter is illustrated in Fig. 3.1. It is the purpose of this chapter not to give a deep overview of the current state-of-the-art in room acoustics modelling, but to discuss the relative merits and disadvantages of the most representative techniques, with the purpose of arriving at a suitable solution to the room acoustics modelling problem. Some of them are revised in more detail due to their relevance and importance for this thesis.

The current chapter is organized as follows: Sec. 3.2 briefly presents the most popular statistical models that are still used presently. The next section deals with wave-based methods, focused on a brief overview of both frequency and time-based methods. Then, in Sec. 3.4 an overview of the state-of-the-art in geometrical methods is presented. Finally the suitability of these methods to analyse the possible contributions these methods can bring is discussed.

\subsection{Statistical methods}

Statistical methods belong to the first group of approximations, the Sabine and Eyring models being the most important. They consider the existence 


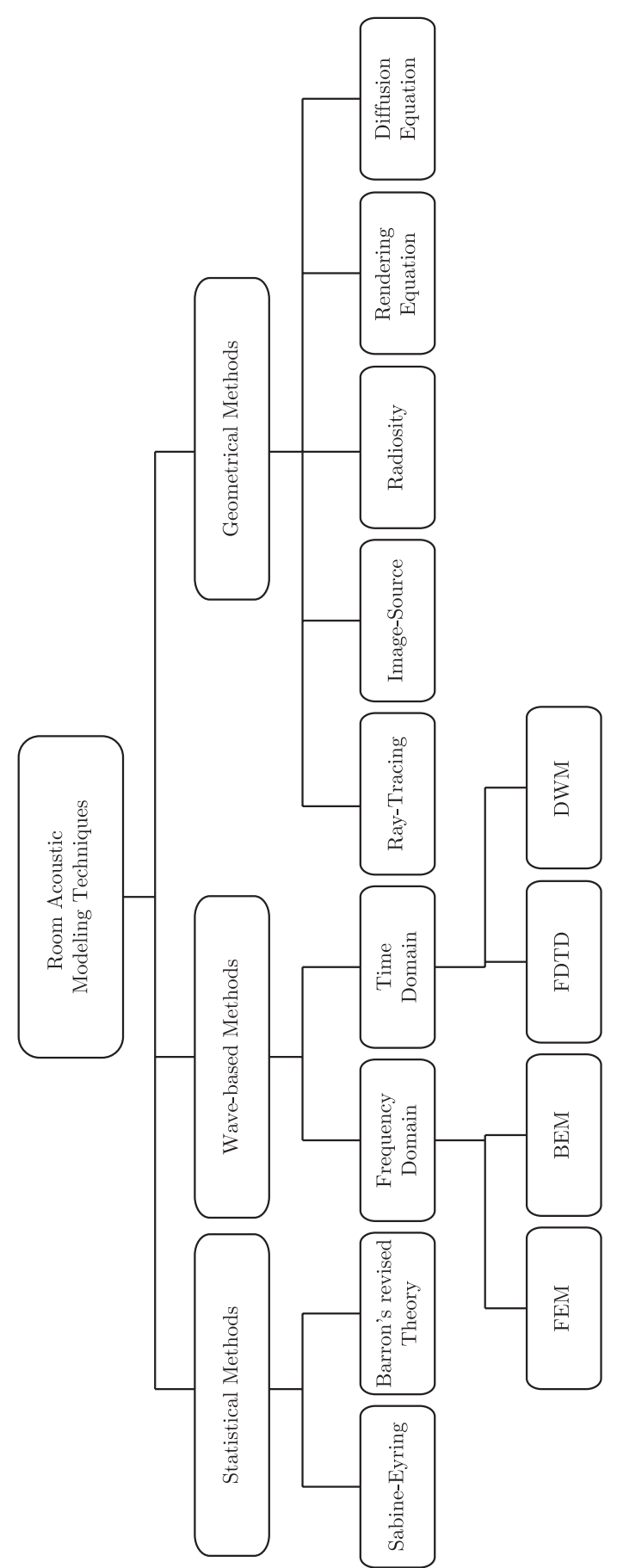

Figure 3.1. A diagram of different approaches to room acoustics modelling. 
of a diffuse sound field in the space, predicting a rough estimation of parameters such as reverberation time, Eq. (2.55) and Eq. (2.57), and sound pressure level Eq. (2.59). The predicted values do not depend on either the source and receiver position or the absorption distribution over walls, because it only considers the size of the room and the amount of absorption. It is important to note that the Sabine and Eyring theories are still used to predict noise levels in reverberant rooms, e.g., factories [Hodgson, 1989]. However, the diffuse field theory is sometimes used to predict sound fields in rooms of every type by practitioners although the fact that the theory is based on assumptions which may limit its applicability is often forgotten (see Sec. 2.3.2.2.1). If the theoretical assumptions are not held, the predictions may not be accurate [Hodgson, 1996a]. For instance, diffuse field theory can not be applied in highly absorptive rooms. Based on this theory, different methods and improvements have been proposed in technical literature over the past few years, e.g., by Hodgson [Hodgson, 1990, 1996b, 1998], Barron's revised theory [Barron, 2000], Kuttruff [Kuttruff, 1985], Cotana [Cotana, 2000] and Thompson et al. [Thompson et al., 1976]. However, these methods are usually specific for one type of room, and have not proved to be accurate over a variety of enclosures. Barron's revised theory [Barron, 2000] is used is this thesis as a reference model in some preliminary experiments (see Sec. 5.3) to evaluate the implementation of a diffusion equation model (see Sec. 3.4.5) by finite-difference schemes.

\subsection{Wave methods}

Wave-based methods are focused in numerically solving the wave equation with adequate boundary conditions to obtain an approximate solution. As was introduced in Sec. 2.3.2.3, this solution shows that each space's region of a room represents a system with the ability to vibrate in its three dimensions, allowing the definition of the room modes. These room modes are of special importance in small rooms and the low frequency range. Some ana- 
lytical solutions for simple shape rooms with simplified boundary conditions (see Sec. 2.3.2.3.2 for an example in a rectangular room) exist. However, in complex shape rooms a numerical analysis is needed.

The wave-based methods can be divided into two main groups, as shown in Fig. 3.1, frequency domain and time domain. In the following sections a qualitative revision of these methods is presented. The most popular wave-based algorithms are: Finite Element Method (FEM), Boundary Element Method (BEM), Finite-Difference Time-Domain method (FDTD), and Digital Waveguide Mesh method (DWM). In general, these methods do a discretisation of time and/or space in order to transform the differential equation into an algebraic system. The numerical solution converges to the analytical one as the intervals of discretisation tend to zero. For instance, the space is divided into small elements or nodes and the size of these elements has to be much smaller than the size of the wavelength for every particular frequency, increasing the computational cost at high frequencies. For practical use, wave models are typically restricted to low frequencies and small spaces [Rindel, 2000].

\subsubsection{Frequency domain}

In this section two of the most important wave-based methods in the frequency domain are revised: FEM and BEM. These methods have been widely used in disciplines such as structural mechanics [Courant, 1943, Dominguez, 1993] and electromagnetism [Chen and Zhou, 1992, Jin, 2002]. In the acoustic literature, they have been used in an innumerable number of applications whose main scope is the analysis of the resonance of acoustic systems [Ihlenburg, 1998, Wrobel and Aliabadi, 2002]. This discretisation is defined for discrete frequencies, resulting in a steady-state sound pressure distribution.

Due to the discrete frequency-based nature of these algorithms, they are not usually applied for room acoustics simulation, but they are very useful and accurate for eigenvalue analysis of the enclosures. Moreover, the use 
of FEM or BEM in room acoustical problems is, at least for the majority of rooms larger than, e.g., vehicle enclosures, up until now limited to a frequency range that is considerably smaller than the range of commonly used room acoustical analysis up to high frequencies. The limiting factor for these methods is the required discretisation of the volume or surface in elements of a size small enough to reconstruct the wave in magnitude and phase at every point of the discretisation domain.

In spite of these limitations, a few applications of these methods in room acoustics exist which makes it worthy to review them in part in the related literature. In the next sections, both methods are briefly revised.

\subsubsection{Finite Element Method}

FEM is mainly used when the interior acoustic field of an enclosure is to be computed. The main advantage of this method lies in its capability for handling problems in complex geometries and inhomogeneous media. In FEM, the space under analysis has to be discretised with elements and the governing equation is expressed as a discrete set of linear equations of these elements. This representation by elements creates matrices that are large but sparsely filled. Each of these elements only interacts with the elements directly adjacent to it. Finally, the system of equations obtained has to be solved.

Although a high number of FEM applications in the wave equation of acoustics exists, the choice of using this method for room acoustics simulation could result in a set of important handicaps. The main limitation lies on the frequency domain: it requires a simulation for each frequency and since the room acoustic properties are usually frequency-dependent, it will considerably increase the computational effort. Moreover it also has another limitation; it requires a very small element size for accurately modelling the entire frequency bandwidth. Six or seven elements per wavelength are required for sufficient sampling rate [van der Geest and McChulloch, 1998, Murphy, 2000]. Therefore FEM is suitable only for small enclosures and 
low frequencies due to its heavy computational requirements.

Despite limitations in the room acoustics field, some analysis of rectangular rooms through FEM have been provided by Wright [Wright, 1995] and Savioja et al. [Savioja et al., 1996a]. Similarly, more realistic rooms have recently been analyzed giving a fair agreement among simulation and measurements in small [Pietrzyk and Kleiner, 1997] and large [Ahnert et al., 2006] rooms.

It important to note that FEM has also been used to model the acoustic diffusion equation model [Picaut et al., 1999b, Valeau et al., 2006], which is one of the main concerns is this thesis. Such technique, which is governed by the general diffusion equation, e.g., such as the heat equation, is an energy transfer model and it will be discussed in Sec. 3.4.5.

\subsubsection{Boundary Element Method}

In BEM, only the boundaries of the space are discretised, therefore, it arises as an alternative method which considerably reduces the computational cost of FEM. However, the matrix for the linear system in BEM is a full matrix, instead of a very sparse one such as in FEM. The BEM consists of applying a FEM method discretisation to a boundary integral equation formulation of the problem. BEM attempts to approximate the solution of the integral equation, that is, the pressure on the boundary $\partial V$, by a simple function, usually a polynomial, at each element. After having computed the acoustic field within the space, the acoustic variables are known at all the node points on the boundary and it can again be evaluated at any point inside the space.

As with FEM, a few examples of its applications to room acoustics simulations can be found. These examples are found in [Bai, 1992], [Wright, 1995], [Savioja et al., 1996a], [Pietrzyk, 1998] and [Osa et al., 2006], where the latter analyses large hall effects through this method. 


\subsubsection{Time Domain}

The wave-based time domain, or discrete-time methods arise as suitable alternative methods, since they are based on discretising the wave equation in the time domain instead of the frequency providing a considerable accuracy, especially at low frequencies. In these techniques the derivatives in the wave equation are replaced by a corresponding finite-difference scheme. It is the most common numerical method used in solving Euler equations.

These methods share a common definition: they discretise the sound propagation's mathematical expressions not only in space, but also in time, giving a recursive algebraic expression which is updated in each time step for every discretised point. Since the first works in the room acoustics field of Botteldooren [Botteldooren, 1995] and Savioja [Savioja et al., 1994] in the mid-nineties, an increasing number of publications and works related to this topic have arisen, where the FDTD and the DWM methods are the most representative ones.

BEM and FEM typically calculate frequency domain responses while FDTD and DWM methods produce time-domain impulse responses which are better for performing auralisation or for predicting room-acoustic quality parameters.

\subsubsection{Finite-Difference Time-Domain}

The FDTD has been successfully applied in many fields such as electromagnetism [Yee, 1966, Taflove, 1988, Kunz and Luebbers, 1993, Taflove, 1995, 1998], acoustics/aeroacoustics [Wang, 1996, Nguyen, 1996, Botteldooren, 1997, Ostashev et al., 2005, Liu and Albert, 2006] and seismology [Graves, 1996, Kristek and Moczo, 2006, Gandomi and Takenaka, 2007].

It was first proposed by Botteldooren [Botteldooren, 1995] in order to numerically solve the Euler equation where the pressure and the particle velocity components are updated in a similar fashion to the electromagnetic approach. Instead of approaching the Euler equation, Savioja et al. [Savioja et al., 1994, 1996b] adapted the suitability of the method to 
the room acoustics simulation through a FDTD discretisation of the wave equation (see Eq. (2.3)). With this approach, the pressure at each node is updated through the surrounding and previous nodes, and the massive storage of the particle velocities is avoided. In those works, the suitability of this method for room acoustics simulation is demonstrated; however, the computational cost is still high for the computation of the total sound field of a moderately-sized room with the total bandwidth of the human hearing. For that reason, the limitation of this method is assumed for low frequencies [Savioja et al., 1994]. However, some research have been done recently to achieve this issue using Graphical Processor Units (GPU) [Adams et al., 2007, Southern et al., 2010, Savioja et al., 2010]. FDTD can also handle frequency-dependent boundary conditions [Escolano et al., 2008, Kowalczyk and van Walstijn, 2011] and broad-band directive sources [Escolano and López, 2009]. Surface diffusion in FDTD is also a challenging topic, a phase grating approach can be applied to room acoustics [Kowalczyk et al., 2011]. Also the performance of sound diffusers in the time domain can be evaluated [Redondo et al., 2007]. To enable efficient and accurate simulation of sound propagation in complex virtual environments, a technique which relies on an adaptive rectangular decomposition of $3 \mathrm{D}$ scenes has been recently presented [Raghuvanshi et al., 2009].

3.3.2.1.1 Finite-Difference Method. The FDTD method can be classified as a special case of the finite-difference method (FDM), a standard method for solving partial differential equations (PDE) [Strikwerda, 1989]. FDM can be applied to a wide range of mathematical physics problems, such as elliptic, parabolic and hyperbolic PDEs. For example, the steadystate heat conduction equation with energy generation, which is called Poisson's equation, is elliptic. Otherwise, the time dependent diffusion equation is parabolic. And in the second-order wave equation case, it is hyperbolic. Each problem requires the selection of the appropriate finitedifference scheme. This method is a numerical technique for solving a differential equation over a given region subject to specified boundary conditions, 
based on a finite difference approach of the involved derivatives of the partial differential equation. When the finite-difference approach is used, the problem domain is discretised so that the values of the unknown dependent variable are considered only at a finite number of nodal points instead of every point over the entire region.

As a contribution of this thesis, FDM is used to implement a numerical solution of the acoustic diffusion equation model (see Chap. 5). The performance of the acoustic diffusion equation model is also analysed with the FDM implementations in Chap. 6.

\subsubsection{Digital Waveguide Mesh}

Digital waveguide models are accurate and efficient methods of modelling a physically complex system. Van Duyne and Smith proposed the use of DWM [Duyne and Smith, 1993, 1995] in room acoustics simulation. A basic waveguide element consists of two delay lines capable of carrying a signal in opposite directions. By interconnecting waveguide elements it is possible for constructing acoustic models based in waveguide networks that can be one, two or three-dimensional. These networks are called meshes and have been successfully used to model the properties of strings, membranes and plates. An extension of these meshes in 3-D allows the creation of an enclosure model to simulate sound propagation [Savioja et al., 1995]. A locally reacting impedance technique is used to model the boundaries' behaviour [Escolano et al., 2009]. The DWM could also be seen as a particular class of finite-difference method [Savioja et al., 1999], with the particularity that it uses a decomposition of the sound pressure waves based on incoming and outgoing components [Duyne and Smith, 1993].

\subsection{Geometrical methods}

So far, several methods have been reviewed in this chapter: statistical methods provide simple formulae but their assumption of a diffuse field restricts 
their use to low reflective rooms and give a non-spatial dependent estimation of parameters like reverberation. On the other hand, wave-based methods analyse the room modes and since they are using the wave equations as a starting point, provide highly accurate results as well as more detailed information of the acoustical properties of the room. Although some improvements have been done in their computational performance, they are still limited to small enclosures and low frequency bands.

Room acoustics prediction involves investigation of both the steadystate and the time-dependent sound fields in a space. In the case of general purpose room acoustic design, the broad-band high frequency solutions and a rough survey of the sound fields are of concern. This allows the application of the so-called geometrical acoustics model as an alternative to the exact wave equation based model and to the diffuse field approach.

As mentioned in Chap. 1, this thesis is focused on analyzing and contributing to these methods, and is particularly centred on the acoustic diffusion equation model. In this section, a summary of the current state of the art of geometrical methods for room acoustics simulation is introduced.

The geometrical methods are based on the quasi-plane wave propagation approach. As it has been introduced in Sec. 2.3.2.1, in this theory the sound propagation's direction can be described with the normal vector of the plane wavefront through the air in straight lines called acoustic rays. Geometrical methods make the assumption that sound wavelengths are significantly smaller than the size of the obstacles found in their path with an analogy to optics theory. This assumption ignores the wave nature of sound, and models in one way or another, the reflections from boundaries. However, this simplification can considerably decrease the computational cost since a PDE problem is directly transformed into an algebraical and/or geometrical problem. Thus, they are valid only for high-frequency sounds. These methods are also called energy-based methods, because the only thing they consider is the energy that was assigned to every ray or portion of a wave, since after all, they use a statistical approach. This means that when 
several sound field components are superimposed, their mutual phase relations are not taken into account; instead, their energy densities or their intensities are simply added. The governing equation of the geometrical optics model is a radiative transfer equation, which describes the physical phenomenon of energy transfer in the form of electromagnetic radiation. As a contribution of this thesis, an acoustic radiative transfer equation is developed to clarify the foundations of the geometrical acoustics methods, especially the acoustic diffusion equation model. This governing equation is derived in Chap. 4.

The most important ray-based methods that have been applied to acoustics are the ray tracing [Krokstad et al., 1968] and image source [Allen and Berkley, 1979] methods. They can trace rays, particles, cones, even pyramids [Farina, 1995, Funkhouser et al., 1998, Stephenson, 1990]. The basic distinction between these methods is the way the reflection paths are calculated. The image source method finds all the possible paths, but it requires a high computational performance and in practice only a set of early reflections is usually computed. Ray tracing applies the Monte Carlo simulation technique to sample these reflections paths and thus gives a statistical result [Allred and Newhouse, 1958]. This technique is more suitable for high order reflection simulations, but there are no guarantees that all the path will be found.

Regarding the boundary conditions, normally these methods assume specular reflections, since the angle of reflection is the same as the angle of arrival like in geometrical optics. However, it is easy to include the boundary absorption issue in a frequency-dependent manner according to the absorption coefficient of the wall. Moreover, it is possible to model sound propagation absorption and air absorption effect. Geometrical acoustics methods generally have an acceptable degree of accuracy in the prediction of different room-acoustic parameters [Vorländer, 1995, Bork, 2000, 2005a,b]. They are also able to achieve the temporal and spatial distribution of reflections, thus they are suitable for auralisation [Lehnert and Blauert, 1992, Kuttruff, 
1993, Kleiner et al., 1993].

In the early years, a considerable disadvantage of these methods lied in the inability to model all wave nature phenomena such as interference, diffuse reflection and diffraction [Savioja, 1999], since they usually appear when wavelengths are similar to the size of obstacles. Nowadays however, some advances have appeared to include these effects and produce highly realistic results, such as diffraction and diffuse reflections [Cox et al., 2006] and the phased geometrical method [Geest and Patzold, 1996, Shin and Ih, 1998]. Several methods to treat the diffuse reflection in geometrical techniques have been implemented, although it is not clear which is more accurate [Lam, 1996]. For instance, a model with some relevance is the acoustical radiosity model [Dalenbäck, 1996], which is an energy transfer model that inherently supposes total diffuse reflection instead of specular reflection. It has been critisized that geometrical room acoustics simulation can only reflect a partial aspect of the acoustical phenomena involved in a room, but this measurement, although partial, is of great importance [Kuttruff, 4th edition, 2000].

Considerable attention has been given to two recently proposed algorithms, the acoustic diffusion equation [Valeau et al., 2006] and the roomacoustic rendering equation [Siltanen et al., 2007a]. The acoustic diffusion equation is an alternative energy approach based on a classical diffusion model. The room-acoustic rendering equation is an unified integral equation that generalizes the geometrical methods in an unique formulation. In Chap. 4, an acoustic radiative transfer model is proposed and both methods are derived from it unifying their foundations and framing their limitations.

In the following sections, the principles of every method, and also some of their drawbacks are overviewed. Special attention is paid to acoustical radiosity in Sec. 3.4.3, the room-acoustic rendering equation in Sec. 3.4.4 and the acoustic diffusion model in Sec. 3.4 .5 because they are related with the contributions of this thesis. In these sections, neither technical nor mathematical foundations are presented, because they will be introduced 
along in the following chapters.

\subsubsection{Ray-tracing}

The ray-tracing method is a general technique from geometrical optics for modeling the path taken by a wave by following rays as they interact with surfaces. This method arises from optical physics [Lipson et al., 1995], with important applications in the computer graphics field [Whitted, 1980, Glassner, 1989] and communications [McJown and Hamilton, 1991, Cátedra et al., 1998, Coleman, 1998, Ji et al., 2001].

The first attempt to use the ray-tracing technique for room acoustics simulation was applied by Krokstad et al. [Krokstad et al., 1968]. They proposed finding propagation paths between a source and a receiver by generating rays emanating from the source position and following them through the environment until a set of rays that reached the receiver had been found. As mentioned above, the initial tendency of this algorithm is to consider only specular reflections following Snell's law, where in each incidence, the incident ray is filtered with its absorbing coefficient, and reflected with the same angle of incidence [Kulowski, 1985]. However, several improvements of the algorithm have included diffusive surfaces [Lam, 1996, Embrechts, 2000] and edge diffraction [Svensson et al., 1999]. Ray-tracing applies the Monte Carlo simulation technique to sample these reflection paths and thus it gives a statistical result [Halton, 1970]. By the use of this technique, higher order reflections can be searched for, though there are no guarantees that all the paths will be found.

Regarding the sources, in principle, the way sound rays are emitted can be either randomized or predefined as a function of the directional characteristics of the sound source [Savioja, 1999]. Therefore, it must be ensured that the generation is almost uniform on the surface of a spherical source [Farina, 1995]. The simple assumption of three random generators for the three components of the vector direction of the ray is not completely correct, as that produces a "cube of rays" instead of a sphere. It is possible 
to cut away the corners of the cube, discarding each vector with a modulus greater than one [Farina, 1995]. If each ray is weighted by an angle dependent function, it is possible to include directivity properties to the source with no increase in computational cost.

The listener positions cannot be considered as a point, since an infinitely small point cannot detect an infinite small ray. For this reason, the receiver should have a finite size. The listeners are typically modelled as spheres or cubes, but they may also be planar. In theory, a listener can be modelled by any shape as long as there are enough rays to penetrate him to achieve statistically valid results. In practice, a sphere is in most cases the best choice, as it provides an omnidirectional sensitivity pattern and it is easy to implement as well.

Some examples of its application and comparison with measurements can be found in technical literature [Hodgson, 1989, Tsingos et al., 2002].

\subsubsection{Image-source}

The concept of image-source has been applied to various field problems such as electromagnetic wave propagation [Balanis, 1997]. In acoustics, the first contribution on the room acoustics field was presented by Allen and Berkley [Allen and Berkley, 1979] and extended to arbitrary geometries with plane walls by Borish [Borish, 1984].

It is assumed that the direction of a sound reflection from a large plane surface follows the same geometrical law, as known from optics. This means that the reflected sound can be interpreted as sound coming from an imagesource behind the reflecting surface (see Fig. 3.2). This principle can be extended to higher order reflections.

The image-source method computes specular reflections paths by considering virtual sources generated by mirroring the location of the audio source, over each boundary surface of the environment. The main advantage of the image-source method is its robustness. It can determine, with 

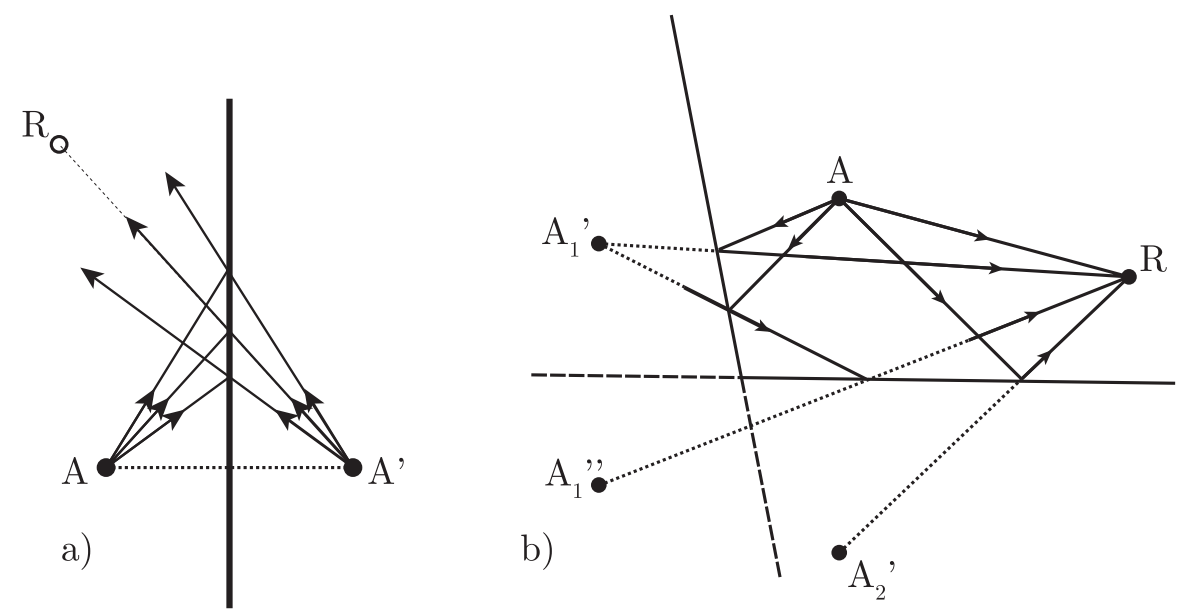

Figure 3.2. (a) Image Source method. (b) First and second order source-image of emitter $A$ and receiver $R$.

a high degree of accuracy in terms of level, arrival time and direction, the early reflections [Rindel, 1995]. Also, the listener can be modelled as a point instead of a volumetric object as in ray tracing. Thus, the results are more accurate if the order of the reflections is high enough. A disadvantage of this method is that it requires very long calculation times for higher order reflections in complex shape rooms. Therefore, its expected computational time increases exponentially, which makes the method ineffective [Stephenson, 1996, Savioja, 1999]. Thus, it is an important challenge to reduce this complexity, i.e. checking visibility. The visible surfaces of each image-source can be calculated by beam-tracing [Stephenson, 1996, Funkhouser et al., 1998]. Each beam is a pyramidal frustum, whose tip is the image-source and whose top is the reflecting surface. Only the surfaces inside these volumes, which are faced toward the image-source, must be taken into account. For this reason image-source models are usually used only for simple rectangular rooms or in such cases where low order reflections are sufficient [Rindel, 1995].

There are also hybrid models, in which ray-tracing and image-source 
method are applied together [Vorländer, 1989, Dalenbäck et al., 1992, Naylor, 1993, Dalenbäck, 1996, Korany et al., 2001, Kuttruff, 2000]. Typically, early reflections are calculated with image-sources due to its accuracy in findings reflections paths, and late reflections are handled with ray-tracing [Naylor, 1993]. Recently, the use of GPUs to accelerate the geometrical algorithm, the diffuse reflections events and edge-diffraction computations is increasing [Savioja et al., 2010].

\subsubsection{Acoustical radiosity}

Acoustical radiosity is a ray-tracing sound field prediction method that assumes diffusely reflecting boundaries [Carroll and Chien, 1977, Nosal et al., 2004a,b, Hodgson and Nosal, 2006]. It is an integral equation that uses Lambert's cosine law to govern diffuse reflection (see Eq. (2.35)).

It was first developed in illumination engineering as the radiative transfer theory [Yamauti, 1926], and later in the thermal-engineering community, as the theory of radiation heat transfer [Siegel and Howell, 1981]. Efficient methods have been developed to implement radiosity in computer graphics as a time-independent technique [Cohen and Wallace, 1993, Ashdown, 1994, Sillion and Puech, 1994]. For an application to acoustics, and to account for the finite speed of sound, Kuttruff developed the time-dependent integral equation in the early 1970s [Kuttruff, 1971, 1976]. Acoustical radiosity has seen much development since that time and has different names, including the integral equation method [Kuttruff, 4th edition, 2000], radiant exchange [Lewers, 1993], and an intensity-based boundary element method [Franzoni and Rouse, 2002]. The radiosity method has been extended to handle specular reflections combined with the image-source method [Korany et al., 2001] or replacing the integral equation with a functional equation [LeBot, 2002].

The underlying concept behind acoustical radiosity is quite simple. The enclosure is divided into elements infinitesimally small in theory, finite in size in practical applications. Each element is considered as both a receiver 
and a secondary source. Considered as a receiver, the energy incident on an element is just the total energy arriving from all sources both primary and secondary in the enclosure. When acting as a secondary source, the element is treated as a diffuse reflector; energy leaving the element is proportional to the energy incident according to the absorption coefficient of the element.

The numerical solution has been applied several times in the literature [Miles, 1984, Kuttruff, 1995, 1997, Kang, 2002] mainly for a rectangular room. E.-M. Nosal et al. [Nosal et al., 2004b] developed improved algorithms and methods for acoustical radiosity for arbitrary polyhedral enclosures, based on a numerical solution to the integral equation. The validity of radiosity for sound field prediction in cubic rooms has been shown [Nosal et al., 2004a]. Experimental evaluation has indicated that radiosity prediction is most accurate in rooms with surfaces that reflect sound somewhat diffusely and at least as well as other energy-based approach with diffuse reflections [Hodgson and Nosal, 2006]. In general, radiosity spreads energy in time, and eliminates any strong reflections from the echogram. This may make it insufficient for auralisation, where strong signals have considerable effect on listener perception. However, parameter values were well predicted by radiosity in the three rooms measured [Hodgson and Nosal, 2006]. It has been shown in these experiments that acoustical radiosity is highly effective in predicting the late part of a decay curve, but radiosity is poor at capturing information about the prominent reflections of a sound field.

Although the time dependence of radiosity in acoustics makes it computationally intensive, methods have been developed that increase its computational efficiency [Nosal et al., 2004b]. Despite these improvements, acoustical radiosity still requires high computational resources both in time and memory.

In Sec. 4.7 a more extended mathematical analysis of the acoustical radiosity method is presented. Also, as a contribution to this thesis, the acoustical radiosity equation is derived from the proposed general theory, the acoustic radiative transfer equation. 


\subsubsection{Room-acoustic rendering equation}

Recently, a general integral equation for room acoustics modelling, called the room-acoustic rendering equation, has been presented [Siltanen et al., 2007a] as a generalization of several well-known geometrical room acoustics modelling methods. The room-acoustic rendering equation was adopted from computer graphics [Kajiya, 1986] as a time-dependent extension, using a direct analogy of parameters between optics and room acoustics.

It can handle arbitrary reflections, both specular and diffuse, representing them via a bidirectional reflectance distribution functions that describes the reflections from different types of materials. Varying the reflectance function in the integral equation different of geometrical room acoustics modelling techniques, such as the image source method, the ray-tracing method and the radiosity method can be derived. In addition, an extension of a progressive radiosity method [Cohen et al., 1988] to handle non-diffuse reflections, called the acoustic radiance transfer method, was proposed to solve the room-acoustic rendering equation [Siltanen et al., 2007a].

In the acoustic radiance transfer method, the surface of the room model is divided into surface elements or patches. The radiance transfer from each patch to every other patch is calculated by using the room-acoustic rendering equation. The interactions between any pair of patches can be presented as simple form factors like in computer graphics [Cohen et al., 1988]. Adding the directional dependence on reflections complicates the calculation by increasing the number of form factors. Moreover, for each patch a time-dependent energy response is stored. These facts make it computationally intensive. This solution is shown to be applicable to model sound propagation in realistic, complex environments having surfaces of various materials [Siltanen et al., 2007b, 2010].

Adding a general correction term to the equations, the method has been extended to handle diffracting edges [Siltanen and Lokki, 2008]. To take into account that the diffusing surface structures not only spread the acoustic 
energy in all directions, but also in time, a time-spreading reflections scheme has been added [Siltanen et al., 2011]. The acoustic radiance transfer models best the late part of the room impulse response where the sound field is already diffuse, but cannot model specular reflections as well as imagesource or ray-tracing methods [Southern et al., 2011, Siltanen et al., 2011].

Several improvements have been made in order to decrease the computational requirements. An implementation of this technique in the frequency domain allows modelling of all frequencies at once [Siltanen et al., 2009, Antani et al., 2011] decreasing the computation time, as the time domain technique requires separate runs for each frequency band. The use of parallel computation by GPUs to solve the acoustic radiance transfer has been recently examined concluding that the frequency-domain implementation is more suitable to parallelise the algorithm [Siltanen et al., 2011].

A preliminary hybrid model combining wave-based and geometrical methods, including acoustic radiance transfer, has recently given promising results [Southern et al., 2011]. In the mid and high-frequency bands this model uses beam-tracing to model the early specular reflections and acoustic radiance transfer to model the more diffusive reflection in the late part of the RIR. In the low-frequency bands the FDTD method is employed.

In Sec. 4.6 a more extended mathematical analysis of the room-acoustic rendering equation is presented with details about functions. Also, as a contribution to this thesis the room-acoustic rendering equation is derived from the proposed general theory, the acoustic radiative transfer equation.

\subsubsection{Acoustic diffusion equation model}

The diffusion equation is a parabolic partial differential equation (PDE) which describes such physical situations as heat conduction in a solid body, population dispersion, and other similar processes. Classical diffusion is an irreversible physical process, in which particles are introduced into a medium that was initially absent, increasing the system entropy formed by 
the scattered particles and the medium in which they diffuse.

In recent times, Valeau et al. [Valeau et al., 2006] have proposed an alternative approach for predicting the sound field in arbitrary rooms with a satisfactory accuracy and with low computation time based on a diffusion model [Ollendorff, 1969, Picaut et al., 1997]. The field is modelled by sound particles [Joyce, 1974] with the same constant energy, propagating along straight lines and striking walls or scattering objects. That diffusion model consists of assuming that the sound propagation in rooms with diffusely reflecting boundaries can be compared with the movement of a single particle moving in a gas and hitting scattering objects [Picaut et al., 1997]. In this way, a partial differential equation is directly enounced in the technical literature describing the diffusion process.

Initially, a mathematical model based on the diffusion equation was used to model simple cubic enclosures [Picaut et al., 1997], to show its agreement with the statistical theory. Then, after comparing predicted results in long rooms with a ray-tracing method, the obtained results showed that the diffusion model can be regarded as an extension of the classical theory of reverberation for non-diffused sound fields [Picaut et al., 1999b].

After that, the acoustic diffusion equation method has been applied to model a wide variety of room types, such as proportionate enclosures and long rooms [Valeau et al., 2006], fitted rooms [Valeau et al., 2007] and even coupled-rooms [Valeau et al., 2004, Billon et al., 2006, Xiang et al., 2009]. This model has also been used to sound field predictions in open spaces like city streets [Picaut et al., 1999a], and in a street canyons [Pollès et al., 2004]. Although specular reflection cannot be applied, an empirical extension to this model for use in non-diffuse reflections has been developed [Foy et al., 2009]. Moreover, to account for sound transmission between two rooms a modification of the diffusion model for room acoustics has been proposed [Billon et al., 2008a]

This approach has been actively studied in recent years due to its high efficiency and flexibility. This model is highly accurate for low absorption 
cases and in predicting the late part of the sound energy decay in a room. However, its theoretical foundations are not clearly defined. In each method, both open [Pollès et al., 2004] and closed [Picaut et al., 1997] enclosures are derived separately. Moreover, although the acoustic diffusion equation has been defined as an energy-based method, its links to geometrical acoustics have not been shown. In prior works, authors used the statistical assumption of ergodicity to equate the local phonon density with the single-particle distribution function [Pollès et al., 2004]. By doing a first order approximation on the particle velocity's magnitude, a diffusion equation that describes the spatial dependence of the energy density as a function of time is obtained [Picaut et al., 1997].

In Sec. 4.5 a more extended mathematical analysis of the acoustic diffusion equation model is presented with details about expressions. Also, as a contribution to this thesis the acoustic diffusion equation model is derived from the proposed general theory, the acoustic radiative transfer equation. The derivation of the diffusion equation is particularly able to offer insight. Also the theoretical assumptions, advantages and limitations are discussed.

\subsection{Discussion}

\subsubsection{About room acoustics simulation methods}

In this chapter, an overview of the modelling techniques used for room acoustics simulation has been presented. Since directly solving the particular inhomogeneous boundary value problem, which represents the room under analysis and is an extremely complex task, simplified models should be used to obtain approximated but accurate solutions.

In general, these methods are classified as statistical, wave-based and geometrical methods. Statistical methods provide simple formulae but their assumption of diffuse field limits their use to some types of rooms only. Wave-based methods numerically solve the wave equation, giving more ac- 
curate results since the wave equation models all sound propagation phenomena, but they are still limited to small enclosures and low frequency band. Special attention have been paid to geometrical methods in this chapter because they are suitable in the case of general purpose room acoustic design. Geometrical methods assume a sound propagation characteristics such as a ray. Under these considerations, the geometrical methods work accurately for high frequencies, since many low frequency phenomena cannot be inherently simulated, such as diffraction and wave interference.

Although the presented geometrical methods have certain similarities, their theoretical foundations are often derived for each method separately. A proper general foundation for geometrical room acoustics modelling is needed. A first approximation has been achieved by the room-acoustic rendering equation [Siltanen et al., 2007a]. Moreover, the theoretical foundations and assumptions of the acoustic diffusion model have to be clarified in order to classify it as a native geometrical model and to carry out comparisons with other geometrical methods. However, the integral room acoustic rendering equation cannot be used to derive the acoustic diffusion equation model.

Within the scope of this thesis, our effort is focused on the development of new room acoustics theories associated with the radiative transfer equation and the diffusion equation. The relation between these two models is that, mathematically, diffusion arises as the long-time large-distance limit of the radiative transfer equation. Moreover, it will be shown that the other classical geometrical methods can be derived from this general model. This is of relevance because it unifies these models and frames their limitations.

\subsubsection{About diffuse reflection}

It is presently admitted that accounting for the scattering or diffusion of sound by surfaces improves room acoustics predictions [Embrechts, 2000]. Investigations published in technical literature by several authors [Hodgson, 1988, 1991, Kuttruff, 4th edition, 2000] have shown that room acoustics 
calculations which are based only on specular reflections overestimate reverberation times in situations where diffusion is significant. Moreover, if diffusion coefficients are defined in the frequency domain and the directivity of scattered energy is modelled, the accuracy in the time domain prediction is improved [Howarth and Lam, 2000].

Ray-tracing and image-source modelling techniques model specular reflections, while acoustical radiosity and the acoustic diffusion equation inherently model diffuse reflections. Although the implementation of the room-acoustic rendering equation inherently handles diffuse and non-diffuse reflections, it models best the late part of the room impulse response where the sound field is already diffuse, however, it cannot model specular reflections so well [Southern et al., 2010, Siltanen et al., 2011]. The assumption of diffuse reflection may not be as limiting as it initially seems. It has been suggested [Kuttruff, 1995, 1997] that the assumption of diffuse reflection is less restrictive than the assumption of specular reflection. It is definitely less restrictive than the assumption of a diffuse sound field. It has been shown that the conversion of specular energy into diffuse energy is irreversible and that all walls produce some diffuse reflection [Hodgson, 1991]. After some reflections, nearly all energy becomes diffusely reflecting [Kuttruff, 1995]. Therefore, ray-tracing and image-source methods are likely more suitable for predicting the early part of the decay curve, while acoustical radiosity, the acoustic radiance transfer model and the acoustic diffusion equation are for the late part. In general, in order to incorporate in the geometrical methods, the diffuse reflection effect becomes a highly computational task. However, the acoustic diffusion equation model is an alternative method that has been actively studied in recent years due to its high efficiency and flexibility [Picaut et al., 1999b, Valeau et al., 2006]. It is based on the classical diffusion partial differential equation. 


\subsubsection{About numerical techniques}

Presently, the FDM and the FEM are widely used for the solution of partial differential equations, e.g, heat, mass and momentum transfer equations. Each method has its advantages depending on the nature of the physical problem to be solved; but there is no better method for all the problems. The accuracy of the FDM can be readily examined by the order of the truncation error in the Taylor's series expansion; but, in the case of FEM no Taylor's series expansion is involved in the formulation to make such assessments [Strikwerda, 1989]. The dimension of the problem is another factor that deserves some consideration. For example, an efficient method for one-dimensional problems may not be so efficient for two or three-dimensional problems. FDM schemes are simple to formulate and can readily be extended to two or three-dimensional problems and require less computational work than the FEMs [Levy and Lessman, 1992]. Furthermore, FDM is very easy to learn and apply for the solving of partial differential equations encountered in the modelling of engineering problems for simple geometries. A major drawback of FDM appears to be in its inability to effectively handle the solution of problems over arbitrarily shaped complex geometries. This is due to the interpolation difficulties between the boundaries and the interior points. This is important, since these interpolations are needed in order to develop finite-difference expressions for nodes next to the boundaries. More recently, with the advent of the numerical grid generation approach [Necati, 1994], the FDM has become comparable to FEM in dealing with irregular geometries, while still maintaining the simplicity of the standard finite-difference method.

In the specific hyperbolic acoustic wave equation problem in rooms, these models are accurate in their results and with ever increasing computer speeds their implementation are becoming easier to achieve. However, due to the computational cost, they are still generally used only for small and low frequencies. Finite Element Analysis methods (FEM and BEM) are well suited for calculating the low frequency room modes, and finite-differences 
methods (FDTD and WDM) have been successfully used to predict the room impulse response.

The main limiting factor of wave-based methods is related to the size of the discretising mesh required for allowing the propagation of a full audio bandwidth signal. This is related with the necessary sampling rate, and it implies a high processing time to obtain a RIR of enough length to extract useful information as well as a large amount of memory resources to store the nodes variables.

The other main limitation is the dispersion error of the simulated waves [Trefethen, 1982]; due to the discretisation of the computational mesh, the actual wave propagation velocity is different from the expected theoretical one.

In the acoustic diffusion equation model case, as far as the author knows, in the revised technical literature, FEM is the only technique that has been used to predict the steady-state and reverberation time values. In this model, the size of the discretising mesh can be larger than the one used for the wave-based models due to the assumptions that have to be done to derive the diffusion equation (see Sec. 4.5.2.1). Therefore, the memory requirements and the elapsed time to run a simulation extremely decrease. In this thesis, an alternative implementation of the acoustic diffusion equation model is developed by means of FDM in the time domain to directly predict the room impulse response, i.e. the energy decay curve, and to calculate the room-acoustic quality parameters.

In summary, the acoustic diffusion equation model is a promising simulation method for general purpose room acoustics design that incorporates diffuse reflections and offers low computational requirements. However, its foundations and assumptions have to be clarified in order to define it as a geometrical model and to analyse its advantages and limitations.

The following chapter (Chap. 4) presents an acoustic radiative transfer model that unifies the geometrical method foundations. This general theo- 
retical model allows the derivation of the acoustic diffusion equation model, together with the other geometrical models revised in the present chapter. 


\section{The Radiative Transfer Model for Sound Field Modelling in Rooms

Sound, JUsT AS LIGHT, is a wave phenomenon. There are several differences between light and sound. In spite of these differences, similarities between light and sound phenomena have made it possible to develop wellestablished modelling techniques in room acoustics, known as geometrical acoustics [Savioja, 1999]. A large number of methods with certain similarities (see Sec. 3.4) exists but their theoretical foundations are often derived for each method separately. Therefore, there is a need for a general foundation for geometrical room acoustics modelling, specially when presently the scientific community has paid attention to an alternative energy-based model that uses a diffusion equation [Picaut et al., 1997]. The classical radiative transfer theory from optics can be used for proposing a general acoustic energy propagation model that unifies the foundations of a wide variety of geometrical acoustics methods including the acoustic diffusion model ${ }^{1}$.

${ }^{1}$ Portions of this chapter constitute the major part of a paper published on the Acta Acustica United with Acustica [Navarro et al., 2010c]. 


\subsection{Introduction}

In this study a theory is developed for acoustic radiative transfer modelling that generalises a wide variety of room acoustics modelling algorithms (some of them described in Sec. 3.4), including the acoustic diffusion equation model.

The classical radiative transfer theory from optics [Chandrasekar, 1960] is used as a general energy propagation theory and adapted for acoustics. It is chosen because both models assume that waves behaves as particles: phonon and photon. The proposed theory incorporates a propagation medium that absorbs and scatters radiation, handling both diffuse and non-diffuse reflections both on boundaries and on objects within the room. From this theory, the concept of sound particles arises for modelling acoustic energy transfer by radiation phenomena occurring in a room. An integrodifferential equation describes the interaction of the sound particles with the medium. The direct solution of this radiative transfer equation is an energy density in the phase space. The sound radiance or angular flux is then obtained, which can immediately leads to the sound intensity and the sound pressure.

Equations of radiative transfer have application in a wide variety of areas, e.g., illuminating engineering [Moon, 1936], radiative heat transfer [Siegel and Howell, 1981], remote sensing [Borel et al., 1991], vision [Wolf, 1992], and computer graphics [Goral et al., 1984]. Since radiative transfer theory is based on conservation of energy [Preisendorfer, 1965], as well as the acoustic wave propagation theory, its application in acoustics remains a valid assumption. Hence, an energy balance model based on the radiative transfer of sound particles is proposed.

As a general model, this room acoustics modelling technique establishes the basis of two recently proposed algorithms, the acoustic diffusion equation (DEM) [Picaut et al., 1997] and the room acoustic rendering equation (REM) [Siltanen et al., 2007a], which also generalizes widely 
used geometrical room acoustics modelling methods, such as the imagesource [Allen and Berkley, 1979], ray-tracing [Krokstad et al., 1968], beamtracing [Funkhouser et al., 2004], and acoustical radiosity [Nosal et al., 2004a]. Considerable attention has been recently given to these methods (DEM and REM). In this chapter, using an analytical approximation and a simplified integral equation, both methods are derived respectively.

The current chapter is organized as follows: firstly in Sec. 4.2 and Sec. 4.3 the environment and the fundamental physical definitions of the theory are respectively presented. Then, the radiative transfer theory is reviewed in Sec. 4.4, its assumptions are described, and the acoustic radiative transfer model for room acoustic simulations is derived. Thirdly, the acoustic diffusion equation model (Sec. 4.5), the room acoustic rendering equation (Sec. 4.6), and acoustical radiosity (Sec. 4.7) are derived from the proposed general model. Concluding this chapter, the underlying assumptions, limitations and advantages of each method are discussed in Sec. 4.8. Finally, a summary of the chapter is presented in Sec. 4.9.

\subsection{Mathematical phase space}

Energy propagation requires an environment, and consequently, modelling requires an environment. The modelling environment consists of the space of the environment, and objects in the space. The surfaces of the objects constitute the modelling geometry, or geometry in short. This section introduces some basic mathematical concepts involving phase space. This definition will be useful in the following sections where a model using the analogy between the propagation of photons and the propagation of sound particles is developed.

To obtain a suitable mathematical description of several room acoustics modelling techniques, it is necessary the definition of phase space as an abstraction for dealing with configurations of particles. The concept of sound particles arises for modelling sound wave propagation, as well as in room 
acoustics in which the sound field is usually modelled by sound particles with the same constant energy, propagating along straight lines and striking walls or scattering objects that absorb and scatter [Joyce, 1974, Kuttruff, 4th edition, 2000]. For simplicity, particles that move with isotropic constant speed $c$ and without internal states are considered. This restricted problem corresponds to assume homogeneous propagation and reflection behavior of sound radiation within the frequency band under analysis. Five degrees of freedom per particle are normally used, corresponding to a 5 dimensional phase space $R^{3} \times S^{2}$, where $R^{3}$ is the Euclidean 3 -space and $S^{2}$ is the unit sphere in $R^{3}$.

The acoustic wave propagation theory is based on conservation of energy, as it has been explained in Sec. 2.2.1. Therefore, to derive a balance equation for particles, the environment and the basic variables of the particles within some fixed volume in phase space must be defined. The room is defined by its total volume $V$, and its total interior surface $S_{\mathrm{t}}$. Let $V \subset R^{3}$ be an arbitrary volume, and let $\Omega \subset S^{2}$ be an arbitrary solid angle, as showed in Fig. 4.1. The cartesian product $V \times \Omega$ is considered, which defines a set of all pairs $(\mathbf{r}, \hat{\mathbf{s}})$ such that $\mathbf{r} \in V$ and $\hat{\mathbf{s}} \in \Omega$. This is directly related with the transport of power along a direction $\hat{\mathbf{s}}$ (where ^ denotes a unit vector) through an absorbing, emitting and scattering infinitesimal volume element $d V$ around the point $\mathbf{r}$.

\subsection{Basic variables of the acoustic radiative trans- fer model}

In this section, the energy transfer concepts are defined. These elementary definitions are applied in later constructions. Since the sound propagation is going to be modelled in this chapter as radiative transfer of sound particles, it is necessary to define a set of basic variables of the theory and its relation with the fundamental acoustic quantities defined in Sec. 2.2.1. Here, the usual notation recognized in radiative optics fields is introduced [Wang and 


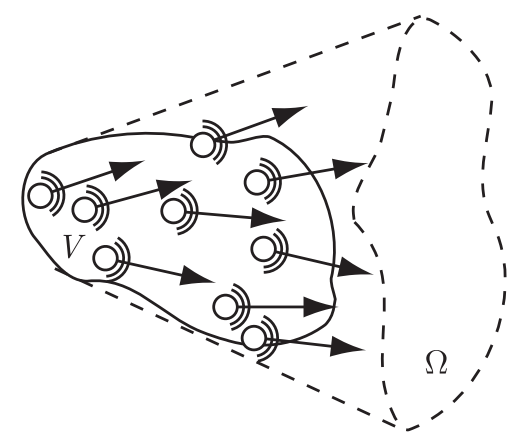

Figure 4.1. A five-dimensional volume in phase space as a result of the cartesian product of a volume $V$ and a solid angle $\Omega$.

$\mathrm{Wu}, 2007]$.

To describe completely a set of propagating particles, we must specify all degrees of freedom of each particle at an instant in time; this corresponds to discrete points in phase space as defined in Sec. 4.2. There are many sound particles moving in the medium at the same time. For a continuum of particles their distribution in the phase space and the evolution of the time distribution is better described in terms of the sound particle phase space density. It is denoted by $N(\mathbf{r}, \hat{s}, t)$ and defined over phase space and time as the number of particles in a differential volume $d V$ about the point $\mathbf{r} \in R^{3}$ moving in a direction within a differential solid angle $d \Omega$ about $\hat{s} \in S^{2}$ at time $t$.

The abstract nature of phase space density makes it quite general, covering almost all particle transport problems from radiative transfer to gas dynamics. For covering the sound particle transport problem with the phase space density, the sound particle energy attribute must be incorporated in it, so that the units in SI are $\mathrm{Jm}^{-3} \mathrm{sr}^{-1}$. Therefore, the total energy in this phase space volume is obtained by

$$
N(t)=\int_{\Omega} \int_{V} N(\mathbf{r}, \hat{\mathbf{s}}, t) d V d \Omega
$$


Our task will be to determine how the number of particles in $V \times \Omega$ changes with time. The sound energy density is then obtained, and integrating this density function over solid angle $\Omega$, gives the average number of sound particles per unit volume $\left(\mathrm{Jm}^{-3}\right)$,

$$
w(\mathbf{r}, t)=\int_{\Omega} N(\mathbf{r}, \hat{\mathbf{s}}, t) d \Omega .
$$

Moreover, the current density or sound energy flow vector $\mathbf{J}\left(\mathrm{Wm}^{-2}\right)$ is defined as the net energy flow per unit area per unit time in a certain direction $\hat{\mathbf{s}}$,

$$
\mathbf{J}(\mathbf{r}, t)=\int_{\Omega} \hat{\mathbf{s}} c N(\mathbf{r}, \hat{\mathbf{s}}, t) d \Omega,
$$

which is the vector counterpart of fluence rate pointing in the direction of the prevalent energy flow.

But rather than focusing on the particles within a volume, the transfer theory emphasizes the energy that propagates through or is emitted from a particular surface $d A$ and the rate at which particles cross it by defining sound radiance $L(\mathbf{r}, \hat{\mathbf{s}}, t)$. The relation between phase space density and radiance is

$$
L(\mathbf{r}, \hat{\mathbf{s}}, t)=c N(\mathbf{r}, \hat{\mathbf{s}}, t),
$$

its units are $\mathrm{Wm}^{-2} \mathrm{Sr}^{-1}$, and it will be the dependent variable in the acoustic radiative transfer equation that will be presented in Sec. 4.4. As Fig. 4.2 shows, it is defined as energy flow per unit normal area per unit solid angle per unit time, where the normal area is perpendicular to the flow direction. In some cases, it is an useful abstraction to allow surfaces to be emitters. Sound that is emitted from boundaries can be described in terms of sound emittance as an area source by $L_{0}\left(\mathbf{r}_{b}, \hat{\mathbf{s}}, t\right)$ where $\mathbf{r}_{b}$ is a surface point.

For taking into account the creation of new particles by sources per unit volume per unit solid angle and per unit time, a phase space source term is expressed by $q(\mathbf{r}, \hat{\mathbf{s}}, t)\left(\mathrm{Wm}^{-3} \mathrm{sr}^{-1}\right)$.

In their travel through the medium the sound particles encounter scattering objects. The collisions between particles are neglected in the radiative 


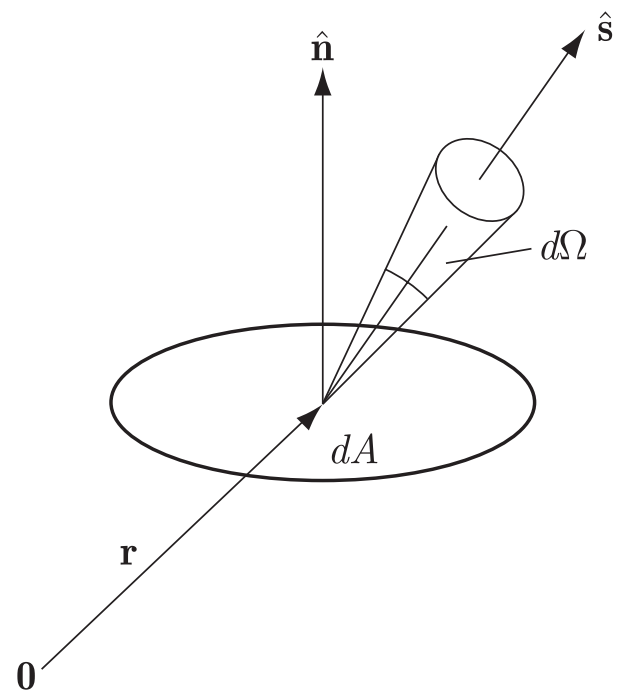

Figure 4.2. Schematic of energy flow through a differential area element $d A$ within a differential solid angle element $d \Omega$

transfer equation assumptions (see Sec. 4.4.1 for details), and only the collisions between particles and scattering objects exist. Scattering objects give rise to both absorption and reflection, such as walls and furniture in a room, or atmospheric absorption. In the radiative transfer theory, the properties of the medium are defined as an attenuation proportional to the path length by absorption, scattering and emission. For simplicity, an average value in the definition of acoustic properties of the medium is assumed. The average straight-line distance experienced by a sound particle between collisions in a highly scattering medium is the sound particle mean free path $\lambda$. Moreover, when a collision with a scattering object occurs, the particle is reflected with the energy $(1-\bar{\alpha})$ and is absorbed with the energy $\bar{\alpha}$, where $\bar{\alpha}$ is defined in acoustics as the room mean sound energy absorption coefficient. Note that it not only refers to absorption of surfaces but also to absorption of objects in the enclosure. Therefore, in our analogy the acoustical properties of the medium are defined as follows. The fraction of incident radiation scattered 
over the unit of the mean free path is designated by $\mu_{s}$,

$$
\mu_{s}=\frac{(1-\bar{\alpha})}{\lambda} .
$$

To take account of the absorption of the scattering objects and the medium, the fraction of incident radiation absorbed over the unit of the mean free path is designated by $\mu_{a}$,

$$
\mu_{a}=m+\frac{\bar{\alpha}}{\lambda}
$$

where $m$ is the air absorption attenuation [Bass et al., 1972] and all values have the dimension of $\mathrm{m}^{-1}$.

\subsection{Acoustic radiative transfer model}

In this section, a mathematical description of the transport of sound particles in a room is proposed by means of an analogy with the radiative transfer theory for transport of photon energy in a scattering medium. Firstly, the general assumptions of the theory are reviewed. Then, the definition of the acoustic radiative transfer equation is described. Finally, in order to obtain a complete model for rooms of complex shape, a general boundary condition definition is presented. Although, the mathematics of this section is based on previous work on light transport in turbid or scattering media [Wang and Wu, 2007, Ishimaru, 1978, Haskell et al., 1994], differences are brought in by introducing several acoustic concepts.

\subsubsection{General assumptions}

Radiative transfer theory is the study of energy transfer in the form of electromagnetic radiation, i.e., light. The propagation of radiation through a medium is mainly affected by absorption, emission and scattering processes.

Sound, just as light, is a wave phenomenon. There are several differences between light and sound, including a much lower propagation speed 
of sound and correspondingly longer wavelengths, and the absence of polarization. However, in spite of these differences, similarities between light and sound phenomena have made it possible to develop well-established modelling techniques in room acoustics, known as geometrical acoustics [Savioja, 1999] (see Sec. 3.4). In a scattering medium light is scattered and absorbed due to inhomogeneities and absorption characteristics of the medium. In room acoustics the sound field is usually modelled by sound particles with the same constant energy, propagating along straight lines and striking walls or scattering objects which absorb and scatter as well [Joyce, 1974, Kuttruff, 4th edition, 2000].

The radiative transfer theory requires some assumptions concerning the nature of the particles, thus, certain applicability conditions must be fulfilled:

- The particles are so small and numerous that their statistical distribution can be treated as a continuum.

- At any point in time a particle is completely characterized by its position and velocity, and internal states such as frequency for sound particles.

- The approximation of geometrical optics, where the wavelength is essentially shorter than the scale of variation of a macrosystem's parameters, uses the concepts of rays or beams for wave propagation in a medium.

- The relationship between the size of individual particles and the working wavelength is arbitrary.

- All processes of the interaction of the external field with a unit volume of a medium are reduced to three acts only - absorption, emission and scattering.

- Particles do not interact with one another; or more precisely, such 
interactions are negligible, i.e. it is necessary to ignore effects such as interference between sound particles.

\subsubsection{Governing acoustic radiative transfer equation}

In this section we approach an acoustic radiative transfer equation focusing on sound particles propagating at a finite speed. In this case, the time dependence cannot be neglected, as is usually done in light energy transport theory. We assume linear acoustics, and the environment for the sound propagation is described by using the concepts established in Sec. 4.2 and Sec. 4.4.1. The sound field carries energy, and propagating sound energy can be compared to propagating electromagnetic radiation by denoting it sound radiance.

Using the quantities defined in Sec. 4.3, the acoustic radiative transfer equation can heuristically be derived from the principle of conservation of energy. A beam of sound can be defined as a set of traveling sound particles moving in a direction within a solid angle $d \Omega$ around a direction $\hat{\mathbf{s}}$ and is usually represented as a pencil as shown in Fig. 4.3. Let us consider a stationary infinitesimal volume element $d V$ and all possible contributions to the energy change in this volume element within an infinitesimal solid angle element $d \Omega$ around the outward direction $\hat{\mathbf{s}}$. In addition, $d \Omega^{\prime}$ is an infinitesimal solid angle element around an incoming direction $\hat{\mathbf{s}}^{\prime}$. The radiative transfer equation states that a beam of sound loses energy through divergence, $d N_{\text {div }}$, and attenuation, including both absorption, $d N_{\text {abs }}$, and scattering, $d N_{\mathrm{l}_{\text {sca }}}$, away from the beam, and gains energy from scattering, $d N_{\mathrm{g}_{\text {sca }}}$, directed towards the beam and sound sources, $d N_{\mathrm{src}}$, in the medium.

The time-resolved transfer equation is a mathematical expression of the build-up of the sound particle density function. Therefore, the changes in energy in the volume element per unit time, $d N$, is expressed by

$$
d N=\int_{\Omega} \int_{V} \frac{1}{c} \frac{\partial L(\mathbf{r}, \hat{\mathbf{s}}, t)}{\partial t} d V d \Omega .
$$




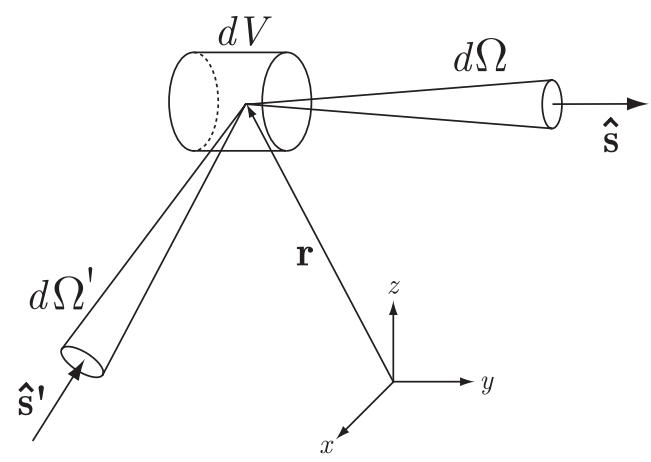

Figure 4.3. Schematic of a stationary infinitesimal volume element.

This rate of change is a result of the balance between the three negative loss contributions and the two positive gain contributions mentioned above. The principle of conservation of energy requires that

$$
d N=-d N_{\mathrm{div}}-d N_{\mathrm{l}_{\mathrm{sca}}}-d N_{\mathrm{abs}}+d N_{\mathrm{g}_{\mathrm{sca}}}+d N_{\mathrm{src}} .
$$

The first right-hand term of Eq. (4.8), $d N_{\text {div }}$, takes into account the sound particles with directions in $\Omega$ that either escape from or enter into the volume $V$ simply by streaming. In general, some infinitesimal surface patches will have positive flows and some negative. More precisely, the change

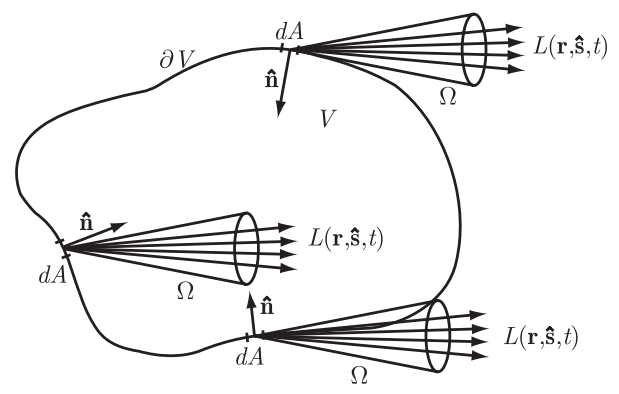

Figure 4.4. Integrating the flux due to $\Omega$ over the surface $\partial V$.

due to streaming is the net flow of particles with directions in $\Omega$ that pass 
through the surface $A_{v}$ of volume $V$, as shown in Fig. 4.4. The streaming through each infinitesimal patch on the surface $d A$ depends only on the flux that is normal $\hat{\mathbf{n}}$ to the patch. The change in energy due to streaming through $A_{v}$ can be expressed by integrating the normal component of the sound radiation due to particles in $\Omega$ over the entire surface $A_{v}$,

$$
d N_{\mathrm{div}}=\int_{\Omega} \int_{A_{v}} L(\mathbf{r}, \hat{\mathbf{s}}, t)(\hat{\mathbf{s}} \cdot \hat{\mathbf{n}}) d A d \Omega .
$$

Using Gauss's theorem we can convert the single surface integral into a volume integral, yielding

$$
d N_{\mathrm{div}}=\int_{\Omega} \int_{V} \hat{\mathbf{s}} \cdot \nabla L(\mathbf{r}, \hat{\mathbf{s}}, t) d V d \Omega .
$$

Here, in addition to Gauss's theorem, we have used the fact that

$$
\nabla \cdot[\hat{\mathbf{s}} L(\mathbf{r}, \hat{\mathbf{s}}, t)]=\hat{\mathbf{s}} \cdot \nabla L(\mathbf{r}, \hat{\mathbf{s}}, t) .
$$

This contribution is due to local beam propagation without collisions with scattering objects; thus, it can exist even in a non-scattering medium.

The next two right-hand terms of Eq. (4.8), $d N_{\mathrm{l}_{\mathrm{sca}}}$ and $d N_{\mathrm{abs}}$, represent the energy change per unit time in the volume element within the solid angle element due to collisions, which can be separated into scattering and absorption events. Thus, the term $d N_{\mathrm{l}_{\mathrm{sca}}}$ takes account of the sound particles scattered from direction $\hat{\mathrm{s}}$ to any another direction,

$$
d N_{\mathrm{lsca}_{\mathrm{sca}}}=\int_{\Omega} \int_{V} \mu_{s} L(\mathbf{r}, \hat{\mathbf{s}}, t) d V d \Omega,
$$

where $\mu_{s}$ is the scattering coefficient defined in Eq. (4.5).

The absorbed sound particles coming from direction $\hat{\mathbf{s}}$ are expressed in the next term, $d N_{\text {abs }}$,

$$
d N_{\mathrm{abs}}=\int_{\Omega} \int_{V} \mu_{a} L(\mathbf{r}, \hat{\mathbf{s}}, t) d V d \Omega,
$$

where $\mu_{a}$ is the absorption coefficient in Eq. (4.6); both air absorption and absorption by scattering objects are included. 
Sound particles gained through scattering from any direction $\hat{\mathbf{s}}^{\prime}$ into $d \Omega$ around direction $\hat{\mathbf{s}}$ per unit time constitute the fourth term $d N_{\mathrm{g}_{\text {sca }}}$,

$$
d N_{\mathrm{g}_{\mathrm{sca}}}=\int_{\Omega} \int_{V} \mu_{s} \int_{\Omega^{\prime}} P\left(\hat{\mathbf{s}}^{\prime}, \hat{\mathbf{s}}\right) L(\mathbf{r}, \hat{\mathbf{s}}, t) d \Omega^{\prime} d V d \Omega
$$

where $P\left(\hat{\mathbf{s}}^{\prime}, \hat{\mathbf{s}}\right)$ is the phase function that represents the probability of particles with propagation direction $\hat{\mathbf{s}}^{\prime}$ being scattered into the solid angle $d \Omega$ around $\hat{\mathbf{s}}$. The simplest phase function is the isotropic phase function where $P\left(\hat{\mathbf{s}}^{\prime}, \hat{\mathbf{s}}\right)=1 / 4 \pi$.

Finally, energy produced by a source in the volume element within the solid angle element per unit time is given by the last term $d N_{\text {src }}$,

$$
d N_{\mathrm{src}}=\int_{\Omega} \int_{V} q(\mathbf{r}, \hat{\mathbf{s}}, t) d V d \Omega,
$$

where $q(\mathbf{r}, \hat{\mathbf{s}}, t)$ is an omnidirectional volume source term.

All six terms derived in Eq. (4.8) entail integration over both $V$ and $\Omega$. The equality must hold for both integrands because $V \times \Omega$ is arbitrary, so the two outer integrals can be removed. Finally, the radiative transfer equation is derived,

$$
\begin{array}{r}
\frac{1}{c} \frac{\partial L(\mathbf{r}, \hat{\mathbf{s}}, t)}{\partial t}=-\hat{\mathbf{s}} \cdot \nabla L(\mathbf{r}, \hat{\mathbf{s}}, t)-\mu_{t} L(\mathbf{r}, \hat{\mathbf{s}}, t) \\
\quad+\mu_{s} \int_{\Omega^{\prime}} P\left(\hat{\mathbf{s}}^{\prime}, \hat{\mathbf{s}}\right) L\left(\mathbf{r}, \hat{\mathbf{s}}^{\prime}, t\right) d \Omega^{\prime}+q(\mathbf{r}, \hat{\mathbf{s}}, t),
\end{array}
$$

where $\mu_{t}$ is the attenuation coefficient as the sum of the absorption coefficient $\mu_{a}$ and the scattering coefficient $\mu_{s}$. The last equation is the acoustic radiative transfer equation. For time-independent responses, valid for steady-state situation, the left-hand side of Eq. (4.16) is zero, which is normally used in computer graphics due to the higher speed of light. It should be noted that Eq. (4.16) is similar to the one obtained in Ref. [Alarcáo and Bento, 2005]. However this chapter presents a more comprehensive and detailed exposition of the acoustic radiative transfer equation. 


\subsubsection{General boundary conditions}

The presence of the gradient operator in Eq. (4.16), makes it a first-order differential equation in the spatial variables for a fixed direction $\hat{\mathbf{s}}$. As such, the equation requires knowledge of the sound radiance at a single point in space into the direction $\hat{\mathbf{s}}$ to be a complete description of the physical situation.

Generally, the point where the sound radiance can be specified lies on the boundary of an enclosure surrounding the medium. Therefore, Eq. (4.16) is only valid away from the boundaries. The sound radiance leaving the surfaces must be determined by solving the boundary conditions.

To express the boundary conditions, let us denote $\partial V$ the entire boundary surface that limits the entire medium $V$, and $\hat{\mathbf{n}}$ the surface normal at point $\mathbf{r}_{b} \in \partial V$. The $S^{2}$ space is partitioned into two hemispheres at each boundary point, designating the hemisphere where $(\hat{\mathbf{n}} \cdot \hat{\mathbf{s}})>0$ the positive hemisphere by $\Omega^{+}$and analogously the negative hemisphere by $\Omega^{-}$(see Fig. 4.5 for details).

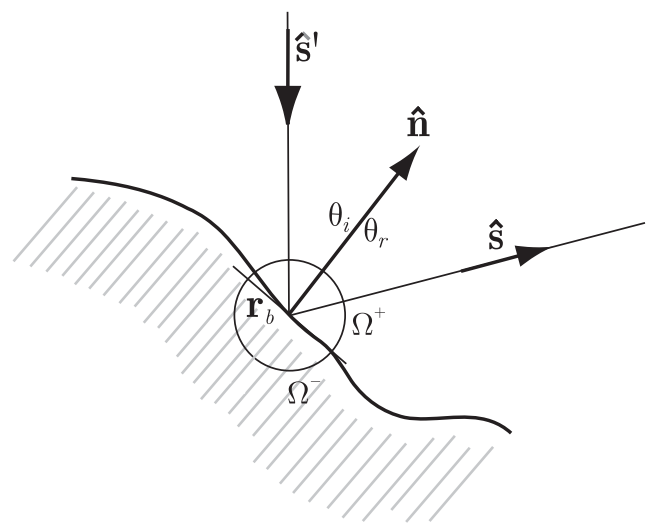

Figure 4.5. The surface $\partial V$ partitions with the set of directions $S^{2}$ at each point $\boldsymbol{r}_{b} \in \partial V$. 
4.5. Derivation of the diffusion equation model from the acoustic radiative transfer equation

A complete definition of the boundary condition involves explicit and implicit boundary conditions. Explicit boundary conditions are independent of sound radiance and take account of the particles that are generated by independent processes $L_{0}\left(\mathbf{r}_{b}, \hat{\mathbf{s}}, t\right)$.

Implicit or reflecting boundary conditions introduce a physical interpretation of the scattering at the boundary: particles created at the boundary are the result of reflections of particles impacting on it. A common type of implicit boundary condition is given by

$$
L\left(\mathbf{r}_{b}, \hat{\mathbf{s}}, t\right)=\int_{\Omega^{-}} R_{F}\left(\mathbf{r}_{b} ; \hat{\mathbf{s}}^{\prime}, \hat{\mathbf{s}}\right) L\left(\mathbf{r}_{b}, \hat{\mathbf{s}}^{\prime}, t\right)\left(\hat{\mathbf{s}}^{\prime} \cdot-\hat{\mathbf{n}}\right) d \Omega^{\prime},
$$

where $R_{F}$ is the surface scattering or reflecting function with units of $\mathrm{sr}^{-1}$ defined as the probability that a particle at $\mathbf{r}_{b}$ moving in the $\hat{\mathbf{s}}^{\prime}$ direction will be reflected into new direction $\hat{\mathbf{s}}$.

Now, we impose a general boundary condition, including both explicit and implicit terms,

$$
\begin{array}{r}
L\left(\mathbf{r}_{b}, \hat{\mathbf{s}}, t\right)=L_{0}\left(\mathbf{r}_{b}, \hat{\mathbf{s}}, t\right) \\
+\int_{\Omega^{-}} R_{F}\left(\mathbf{r}_{b} ; \hat{\mathbf{s}}^{\prime}, \hat{\mathbf{s}}\right) L\left(\mathbf{r}_{b}, \hat{\mathbf{s}}^{\prime}, t\right)\left(\hat{\mathbf{s}}^{\prime} \cdot-\hat{\mathbf{n}}\right) d \Omega^{\prime} .
\end{array}
$$

Finally, Eq. (4.18) together with the acoustic radiative transfer equation Eq. (4.16) compose the system of equations for sound particles transport in the acoustic radiative transfer model.

\subsection{Derivation of the diffusion equation model from the acoustic radiative transfer equation}

\subsubsection{Technical and mathematical foundations of the acoustic diffusion equation model}

In this section, the mathematical concerns about the acoustic diffusion model, both the governing and boundary condition equation are presented 
previously to its derivation from an approximation of the acoustic radiative transfer model.

\subsubsection{Governing diffusion equation}

In previous works, the acoustic diffusion model is based on the assumption that the sound propagation in rooms with diffusely reflecting boundaries can be modelled by analogy with propagation of gas particles in a diffusing fluid. A partial differential equation for this phenomenon is available in the technical literature. The scattering objects take the place of the walls and reflect the sound diffusely in the volume. In this case, the sound energy flow vector $\mathbf{J}(\mathbf{r}, t)$ within a room, is related to the gradient of the sound energy density $w(\mathbf{r}, t)$, according to Fick's law [Morse and Feshbach, 1953, Picaut et al., 1997],

$$
\mathbf{J}(\mathbf{r}, t)=-D \nabla w(\mathbf{r}, t),
$$

where $D$ is the diffusion coefficient, which is expressed as

$$
D=\frac{\lambda c}{3}
$$

It is important to note that, the diffusion coefficient $D$ used here is not the proportion of diffuse reflections at the room surfaces [Beranek, 1954]. This is a term that takes account of the room geometry and shape through the mean free path. In classical acoustic theory [Pierce, 2007, Kosten, 1960], the mean free path for empty room is given by Eq. (2.56). For a medium containing scattering objects the mean free path for the propagation of particles should be evaluated as in Ref. [Valeau et al., 2007].

With an omnidirectional sound source $q_{0}(\mathbf{r}, t)$ in the volume, the sound energy density $w(\mathbf{r}, t)$ distribution can be expressed as [Valeau et al., 2006],

$$
\begin{aligned}
\frac{\partial w(\mathbf{r}, t)}{\partial t} & =-\nabla \cdot \mathbf{J}(\mathbf{r}, t) \\
& =D \nabla^{2} w(\mathbf{r}, t)-\sigma w(\mathbf{r}, t)-m c w(\mathbf{r}, t)+q_{0}(\mathbf{r}, t) \text { in } V(4.21)
\end{aligned}
$$

where $\sigma w(\mathbf{r}, t)$ describes the energy loss per unit volume due to absorption at the room boundaries, with $\sigma=\bar{\alpha} c / \lambda$. The term $c m w(\mathbf{r}, t)$ accounts for 
4.5. Derivation of the diffusion equation model from the acoustic radiative transfer equation

air dissipation within the room with $m$ being the absorption coefficient of air [Bass et al., 1972, Billon et al., 2008b]. These absorption losses are frequency dependent, both room boundaries and air.

\subsubsection{Boundary conditions}

If the diffusion equation should be used in a room of arbitrary shape, the equivalent scattering medium has to be bounded with appropriate boundary conditions. In previous works two kinds of boundary conditions have been presented, a homogeneous Neumann boundary condition [Valeau et al., 2006] where sound energy cannot escape from the room boundaries (its validity is discussed in [Jing and Xiang, 2008]); and a more realistic mixed boundary condition for general situations which allows energy exchanges with the boundaries $\partial V$.

In order to use the mixed boundary conditions, the absorption should occur on room surfaces rather than in the volume. To model the local effects on the sound field induced by different absorbing materials on surfaces, the following mixed boundary conditions have been published [Valeau et al., 2006]

$$
\mathbf{J}(\mathbf{r}, t) \cdot \hat{\mathbf{n}}=-D \frac{\partial w}{\partial \hat{\mathbf{n}}}=A_{x} c w(\mathbf{r}, t) \text { on } \partial V,
$$

where $A_{x}$ is an exchange factor denoted in the following as the absorption factor, $\hat{\mathbf{n}}$ is the normal direction of the surface, $\mathbf{J}$ is the energy flux vector and $\partial V$ makes reference to the boundary conditions of the volume $V$. The latter equation makes possible to assign different absorption coefficients to each individual walls.

This mixed boundary conditions give rise to the following system of equations [Valeau et al., 2006, Billon et al., 2008b],

$$
\begin{array}{r}
\frac{\partial w(\mathbf{r}, t)}{\partial t}-D \nabla^{2} w(\mathbf{r}, t)+m c w(\mathbf{r}, t)=q_{0}(\mathbf{r}, t) \text { in } V, \\
D \frac{\partial w(\mathbf{r}, t)}{\partial \hat{\mathbf{n}}}+c A_{x} w(\mathbf{r}, t)=0 \text { on } \partial V,
\end{array}
$$


which is a second order parabolic partial differential equation with mixed boundary conditions. The absorption factor $A_{x}=A_{x}(\mathbf{r}, \alpha)$ in Eq. (4.24) (where $\alpha$ is the surface absorption coefficient) can describe rooms with low [Valeau et al., 2006] or high absorption [Jing and Xiang, 2007, Billon et al., 2008c]. If the room walls are made of $N$ surfaces with constant absorption $\alpha_{i}$, then absorption factor $A_{x}$ to each surface portion $i$ can be written

$$
A_{x}=A_{S}(\mathbf{r}, \alpha)=\frac{\alpha(\mathbf{r})}{4} .
$$

The subscript $S$ of $A_{S}$ is used to denote Sabine absorption, which is in accordance with diffuse sound-field theory [Kuttruff, 4th edition, 2000]. Sabine absorption factor has been found to be accurate only for modelling room with low absorption about mean effective absorption values below 0.2 [Valeau et al., 2006, Picaut et al., 1999b,a]. In order to improve the predictions in higher absorbing rooms, where Sabine's theory is no longer valid, the Eyring absorption factor has been defined [Jing and Xiang, 2007, Billon et al., 2008c] as follows

$$
A_{E}(\mathbf{r}, \alpha)=\frac{-\log _{e}(1-\alpha(\mathbf{r}))}{4}
$$

Another derivation of this boundary conditions based on a boundary condition in light diffusion in media, that avoid the singularity within the Eyring factor when the absorption coefficient becomes 1.0, is called modified absorption factor [Jing and Xiang, 2008].

$$
A_{M}(\mathbf{r}, \alpha)=\frac{\alpha(\mathbf{r})}{2(2-\alpha(\mathbf{r}))}
$$

However, the main advantage of this boundary condition is that it has been demonstrated to be more accurate for higher absorption coefficient, including an extension of the range of absorbing coefficients up to 0.7 [Jing and Xiang, 2008]. 
4.5. Derivation of the diffusion equation model from the acoustic radiative transfer equation

\subsubsection{Relation to the acoustic radiative transfer model}

In this section, the acoustic diffusion equation model is derived from an approximation to the acoustic radiative transfer model.

\subsubsection{Governing diffusion equation}

In spite of the developments and the potential of the acoustic diffusion equation model, there is a need for a clear, complete exposition of the theory and the assumptions behind the method. The acoustic radiative transfer equation is difficult to solve since it has six independent variables $\left(\mathbf{r}=[x, y, z] \in R^{3}, \hat{\mathbf{s}}=[\theta, \phi] \in S^{2}, t\right)$. By making appropriate assumptions about the behavior of sound particles in a scattering medium, the number of independent variables can be reduced. Some different approximations have been developed in several scientific fields trying to simplify the radiative transfer equation [Rohsenow et al., 1998]. One of the most useful is the diffusion approximation, since it can be solved analytically for simple geometries, and numerically for complex enclosures [Ishimaru, 1978], and here is adapted for room acoustics modelling.

Two main assumptions are needed for the application of the diffusion theory to the radiative transfer equation:

1. The scattering density must be high, and the reflection of energy must dominate over absorption. This means that after numerous diffuse reflections the radiance becomes nearly isotropic. This assumption is sometimes called directional broadening.

2. In a primarily scattering medium the time for substantial energy flux changes is much longer than the time it takes to traverse one mean free path. Thus, over one transport mean free path, the fractional change in the sound energy flow vector is much less than unity [Morse and Feshbach, 1953]. This property is sometimes called temporal broadening.

As pointed out in Sec. 3.4.5, other derivations of the acoustic diffusion 
equation model have been presented in prior literature where directional and temporal broadening have also been assumed in one way or the other. In the diffusion equation proposed by Picaut et al. [Picaut et al., 1997] for room acoustics, a first order approximation on the velocity's magnitude of the particle density function is used. For the expansion to be valid, the scattering obstacles are assumed to be in more quantity than the sound particles, that is in accordance with the directional broadening. Moreover, the variation in the energy density and the energy flow per mean free path must be small, which is the temporal broadening assumption. Otherwise, in an alternative approximation of the diffusion equation based on the transport equation [Pollès et al., 2004, 2005], that is used for predicting sound propagation in a street canyon, the two planes that compose the street are close to each other, increasing the particle frequency collision on the planes. It should be noted that this assumption is the same as the directional broadening. Moreover, the time variable is rescaled, which is related to the temporal broadening

A formal method of solving the resulting integro-differential equation is to expand the sound radiation in appropriate function series. In order to obtain the acoustic diffusion equation, this is done by spherical harmonics $Y_{n, m}$ [Williams, 1999]. Thus the position and directional variables can be separated into new functions.

Expanding the sound radiance function $L(\mathbf{r}, \hat{\mathbf{s}}, t)$ yields:

$$
L(\mathbf{r}, \hat{\mathbf{s}}, t) \approx \sum_{n=0}^{1} \sum_{m=-n}^{+n} L_{n, m}(\mathbf{r}, t) Y_{n, m}(\hat{\mathbf{s}}),
$$

where $L_{n, m}$ are the expansion coefficients. In the diffusion approximation, only the lowest order in the expansion are used, to meet the directional broadening assumption. The term for $n=0$ and $m=0$ represents the isotropic component, whereas the terms for $n=1$ and $m=0, \pm 1$ represent the anisotropic component. 
4.5. Derivation of the diffusion equation model from the acoustic radiative transfer

Substituting Eq. (4.28) into Eq. (4.2) yields

$$
w(\mathbf{r}, t)=\frac{4}{c} \pi L_{0,0}(\mathbf{r}, t) Y_{0,0}(\hat{\mathbf{s}}),
$$

Multiplying Eq. (4.28) by $\hat{s}$ and substituting it into Eq. (4.3) gives

$$
\mathbf{J}(\mathbf{r}, t) \cdot \hat{\mathbf{s}}=\frac{4 \pi}{3} \sum_{m=-1}^{1} L_{1, m}(\mathbf{r}, t) Y_{1, m}(\hat{\mathbf{s}}),
$$

Combining Eqs. (4.29) and (4.30) with the expansion of $L(\mathbf{r}, \hat{\mathbf{s}}, t)$ Eq. (4.28) gives

$$
L(\mathbf{r}, \hat{\mathbf{s}}, t)=\frac{c}{4 \pi} w(\mathbf{r}, t)+\frac{3}{4 \pi} \mathbf{J}(\mathbf{r}, t) \cdot \hat{\mathbf{s}} .
$$

The source function can also be expanded in spherical harmonics,

$$
q(\mathbf{r}, \hat{\mathbf{s}}, t)=\frac{1}{4 \pi} q_{0}(\mathbf{r}, t)+\frac{3}{4 \pi} \mathbf{q}_{1}(\mathbf{r}, t) \cdot \hat{\mathbf{s}} .
$$

Now, substituting this diffusion approximations Eq. (4.31) and Eq. (4.32) into the acoustic radiative transfer equation Eq. (4.16) and integrating over the full solid angle gives the following scalar differential equation,

$$
\frac{1}{c} \frac{\partial w(\mathbf{r}, t)}{\partial t}+\frac{1}{c} \nabla \cdot \mathbf{J}(\mathbf{r}, t)+\mu_{a} w(\mathbf{r}, t)=\frac{1}{c} q_{0}(\mathbf{r}, t) .
$$

Finally, substituting Eqs. (4.31) and (4.32) into Eq. (4.16), multiplying both sides by $\hat{\mathrm{s}}$ and integrating over all solid angle yields the vector differential equation

$$
\frac{1}{c^{2}} \frac{\partial \mathbf{J}(\mathbf{r}, t)}{\partial t}+\frac{1}{3} \nabla w(\mathbf{r}, t)+\frac{1}{3 D} \mathbf{J}(\mathbf{r}, t)=\frac{1}{c} \mathbf{q}_{1}(\mathbf{r}, t),
$$

where $D$ is the diffusion coefficient defined as

$$
D=\frac{c}{3 \mu_{t}}=\frac{c}{3\left(\frac{1}{\lambda}+m\right)} \approx \frac{\lambda c}{3} .
$$


Note that this formulation of the diffusion coefficient is in accordance with the extension of the diffusion model presented in Ref. [Billon et al., $2008 \mathrm{~b}]$, and the approximation in the equation corresponds to neglecting the air absorption, in accordance with [Valeau et al., 2006] (see Eq. (4.20)).

It should be emphasized that in this approach the phase function in Eq. (4.16) is regarded as isotropic $P\left(\hat{\mathbf{s}}^{\prime}, \hat{\mathbf{s}}\right)=1 / 4 \pi$,

$$
\int_{4 \pi} P\left(\hat{\mathbf{s}}^{\prime}, \hat{\mathbf{s}}\right) d \Omega^{\prime}=1
$$

therefore, all boundaries are assumed to be diffusely reflecting in order to fulfill directional broadening.

The source term $q(\mathbf{r}, \hat{\mathbf{s}}, t)$ is also supposed to be isotropic, i.e. omnidirectional, therefore $\mathbf{q}_{1}(\mathbf{r}, \hat{\mathbf{s}}, t)=0$. Finally, assuming that the fractional change in energy flow within a mean free time (reverse of mean free path) is small, being this supposition what is previously called temporal broadening, Eq. (4.34) in the steady state yields an expression for the sound energy flow vector that is analogous to Fick's law (see Eq. (4.19)). Even when the source varies in time, Eq. (4.19) is a good approximation if temporal broadening can be assumed [Morse and Feshbach, 1953].

The two relations between the sound energy density and the sound energy flow vector (see Eqs. (4.33) and (4.19)) can be combined, which leads to the diffusion equation,

$$
\frac{\partial w(\mathbf{r}, t)}{\partial t}-D \nabla^{2} w(\mathbf{r}, t)+c \mu_{a} w(\mathbf{r}, t)=q_{0}(\mathbf{r}, t),
$$

where the diffusion coefficient is supposed to be space-invariant. Note that this expression is in accordance with Eq. (4.21) using the definition of $\mu_{a}$ (see Eq. (4.6)).

In this derivation of the diffusion equation from the acoustic radiative transfer equation, two approximations have been made,

- The directional broadening permits the expansion of the radiance to be limited to the first-order spherical harmonics. 
4.5. Derivation of the diffusion equation model from the acoustic radiative transfer equation

- The temporal broadening ensures that the fractional change in $\mathbf{J}(\mathbf{r}, t)$ in one transport mean free path is much less than unity.

The diffusion equation model can now be regarded as a geometrical acoustics method, as well as ray-tracing and image-source models.

\subsubsection{Boundary conditions}

The diffusion equation derived in previous Sec. 4.5.2.1 is valid for an infinite scattering medium. Consideration of boundary conditions permits the use of the diffusion equation for characterising sound propagation in a bounded medium.

In what follows two kinds of boundary conditions are considered, the homogeneous Neumann boundary condition and the mixed boundary condition but from the point of view of the diffusion approximation presented above. Fig. 4.5 illustrates the coordinates in this section for sound reflection and absorption on a boundary.

4.5.2.2.1 Homogeneous boundary conditions. The absorption of the surfaces of the room are included in the diffusion equation expressed on Eq. (4.37) by the term $\mu_{a}$. This term contains the loss of energy in the room per unit volume and per unit time associated with the mean room absorption coefficient $\bar{\alpha}$. Therefore, the boundary condition should impose no flux of sound energy through the room surfaces. This boundary condition is expressed by taking into account that the direction-integrated sound radiation function at the boundary and directed towards the medium is equal to the directionintegrated sound radiation function at the boundary and directed out of the medium. In other words, total reflection yields

$$
\int_{\Omega^{+}} L\left(\mathbf{r}_{b}, \hat{\mathbf{s}}, t\right)(\hat{\mathbf{s}} \cdot \hat{\mathbf{n}}) d \Omega=\int_{\Omega^{-}} L\left(\mathbf{r}_{b}, \hat{\mathbf{s}}^{\prime}, t\right)\left(\hat{\mathbf{s}}^{\prime} \cdot-\hat{\mathbf{n}}\right) d \Omega^{\prime}
$$

The diffusion approximation gives an expression for the sound radiation 
function Eq. (4.31), and the boundary condition becomes after integration

$$
\frac{w\left(\mathbf{r}_{b}, t\right)}{4}+\frac{1}{2 c} \mathbf{J}\left(\mathbf{r}_{b}, t\right) \cdot \hat{\mathbf{n}}=\frac{w\left(\mathbf{r}_{b}, t\right)}{4}-\frac{1}{2 c} \mathbf{J}\left(\mathbf{r}_{b}, t\right) \cdot \hat{\mathbf{n}} .
$$

Substituting Fick's law, Eq. (4.19), gives

$$
\mathbf{J}\left(\mathbf{r}_{b}, t\right) \cdot \hat{\mathbf{n}}=-D \nabla w\left(\mathbf{r}_{b}, t\right) \cdot \hat{\mathbf{n}}=0,
$$

therefore,

$$
\frac{\partial w\left(\mathbf{r}_{b}, t\right)}{\partial \hat{\mathbf{n}}}=0 \text { on } \partial V
$$

This derivation yields Eq. (4.41) for boundaries as the homogeneous model described in Valeau et al. [Valeau et al., 2006].

4.5.2.2.2 Mixed boundary conditions. The boundary condition introduced in Sec. 4.5.2.2.1, has no real physical meanings for the application to complex rooms, because it assumes that the absorption occurs in the volume rather than on the room surfaces. Also, a boundary condition that makes it possible to define different absorption coefficients for each surface that forms the room is needed for simulating realistic enclosures. To model the local effects on the sound field induced by different absorbing materials on surfaces, let us adopt the following mixed boundary conditions based on partial-current boundary condition in light diffusion [Haskell et al., 1994].

This boundary condition is derived introducing the surface reflectance factor $R_{F}$ in Eq. (4.38),

$$
\int_{\Omega^{+}} L\left(\mathbf{r}_{b}, \hat{\mathbf{s}}, t\right)(\hat{\mathbf{s}} \cdot \hat{\mathbf{n}}) d \Omega=\int_{\Omega^{-}} R_{F}\left(\mathbf{r}_{b} ; \hat{\mathbf{s}}^{\prime}, \hat{\mathbf{s}}\right) L\left(\mathbf{r}_{b}, \hat{\mathbf{s}}^{\prime}, t\right)\left(\hat{\mathbf{s}}^{\prime} \cdot-\hat{\mathbf{n}}\right) d \Omega^{\prime} .
$$

Evaluating the integrals after substitution with the diffusion approximation gives

$$
\frac{w\left(\mathbf{r}_{b}, t\right)}{4}+\frac{1}{2 c} \mathbf{J}\left(\mathbf{r}_{b}, t\right) \cdot \hat{\mathbf{n}}=R_{F}\left(\mathbf{r}_{b}\right) \frac{w\left(\mathbf{r}_{b}, t\right)}{4}-R_{F}\left(\mathbf{r}_{b}\right) \frac{1}{2 c} \mathbf{J}\left(\mathbf{r}_{b}, t\right) \cdot \hat{\mathbf{n}},
$$


where $R_{F}\left(\mathbf{r}_{b}\right)$ is assumed to be independent of the angle of incidence following the diffusion approximation assumptions. Applying Fick's law gives

$$
c w\left(\mathbf{r}_{b}, t\right)=-2 D \frac{1+R_{F}\left(\mathbf{r}_{b}\right)}{1-R_{F}\left(\mathbf{r}_{b}\right)} \frac{\partial w\left(\mathbf{r}_{b}, t\right)}{\partial \hat{\mathbf{n}}} .
$$

By analogy with room acoustics, the reflectance factor or energy based reflectivity can be expressed using the absorption coefficient as $\left(1-\alpha\left(\mathbf{r}_{b}\right)\right)$. Therefore, a simple operation after substitution yields

$$
D \frac{\partial w\left(\mathbf{r}_{b}, t\right)}{\partial \hat{\mathbf{n}}}+\frac{c \alpha\left(\mathbf{r}_{b}\right)}{2\left(2-\alpha\left(\mathbf{r}_{b}\right)\right)} w\left(\mathbf{r}_{b}, t\right)=0 \text { on } \partial V .
$$

This equation is called the modified boundary conditions and along with Eq. (4.37) without the absorption term $\mu_{a}$ in the volume they lead to a system of equations for the acoustic diffusion model within the volume and the boundaries (see Eq. (4.23) and Eq. (4.24)).

Note that Eq. (4.45) agrees with the $A_{x}$ previously derived for acoustic purpose by Jing and Xiang [Jing and Xiang, 2008] (see Eq. (4.27)).

\subsection{Derivation of the room-acoustic rendering equa- tion}

In this section the room-acoustic rendering equation is presented previously to its derivation from the acoustic radiative transfer model.

\subsubsection{Technical and mathematical foundations of the room-acoustic rendering equation}

As reviewed in Sec. 3.4.4, a general geometrical room acoustics modelling method, called the room-acoustic rendering equation, has been recently presented [Siltanen et al., 2007a]. The formulation used by Siltanen et al. is adapted to notation and variables defined in Sec. 4.3 for clarity. This integral equation based method can handle both diffuse and non-diffuse reflections. 
The integral equation describes the time-dependent sound radiance $L\left(\mathbf{r}_{b}, \hat{\mathbf{s}}, t\right)$ leaving a point on a surface $\mathbf{r}_{b}$ in direction $\hat{\mathbf{s}}$ as a combination of the emitted time-dependent radiance $L_{0}\left(\mathbf{r}_{b}, \hat{\mathbf{s}}, t\right)$ and, the amount of the reflected time-dependent radiance $L\left(\mathbf{r}_{b}^{\prime}, \hat{\mathbf{s}}^{\prime}, t-\frac{\left(\mathbf{r}_{b}-\mathbf{r}_{b}^{\prime}\right) \cdot \hat{\mathbf{s}}^{\prime}}{c}\right)$ arriving from all visible surfaces,

$$
\begin{array}{r}
L\left(\mathbf{r}_{b}, \hat{\mathbf{s}}, t\right)=L_{0}\left(\mathbf{r}_{b}, \hat{\mathbf{s}}, t\right) \\
+\int_{\partial V} R\left(\mathbf{r}_{b} ; \hat{\mathbf{s}}^{\prime}, \hat{\mathbf{s}}\right) L\left(\mathbf{r}_{b}^{\prime}, \hat{\mathbf{s}}^{\prime}, t-\frac{\left(\mathbf{r}_{b}-\mathbf{r}_{b}^{\prime}\right) \cdot \hat{\mathbf{s}}^{\prime}}{c}\right) d A^{\prime},
\end{array}
$$

where term $R\left(\mathbf{r}_{b} ; \hat{\mathbf{s}}^{\prime}, \hat{\mathbf{s}}\right)$ is the so-called reflection kernel, that combines together the reflectance factor $R_{F}\left(\mathbf{r}_{b} ; \hat{\mathbf{s}}^{\prime}, \hat{\mathbf{s}}\right)^{2}$, the propagation operator, the geometry term, and the visibility term.

In this section, it is shown that the room-acoustic rendering equation can be derived as a particular solution of the acoustic radiative transfer equation. There are general solutions of the radiative transfer equation in integral form, mainly when the quasi-steady state is assumed, something that is usually done in electromagnetic works. However in this section, a simplified time-dependent analytical solution is obtained in integral form with boundary conditions included.

\subsubsection{Relation to the acoustic radiative transfer model}

As mentioned above, the radiative transfer equation represents a radiant energy balance equation in an infinitesimal volume element. The rendering equation method is also based on a radiation balance equation. However, in contrast to the acoustic radiative transfer equation, the room-acoustic rendering equation balances incoming and outgoing radiation fluxes on infinitesimal surface elements [Gerstl and Borel, 1992]. Another important difference between them is that the acoustic radiative transfer equation includes in the propagation medium both absorption and scattering, whereas the room-acoustic rendering equation does not inherently take into account

\footnotetext{
${ }^{2}$ some authors denominate this term as the bidirectional reflectance distribution function [Siltanen et al., 2007a].
} 
the scattering media. However, it should be noted that scattering and attenuation by objects might be artificially modelled using the room-acoustic rendering equation as room surfaces or boundaries with some geometry. Otherwise, absorption that causes sound attenuation due to air particles is taken into account in the room-acoustic rendering equation, and thus the absorption term in Eq. (4.16) is $\mu_{a}=m$.

Due to the differences mentioned above, let us begin the derivation of the room-acoustic rendering equation starting from the acoustic radiative transfer equation, Eq. (4.16) ignoring the integral scattering term, but with the air absorption term included,

$$
\frac{1}{c} \frac{\partial L(\mathbf{r}, \hat{\mathbf{s}}, t)}{\partial t}+\hat{\mathbf{s}} \cdot \nabla L(\mathbf{r}, \hat{\mathbf{s}}, t)=-m L(\mathbf{r}, \hat{\mathbf{s}}, t)+q(\mathbf{r}, \hat{\mathbf{s}}, t) .
$$

This is an inhomogeneous first order partial differential equation. It can be interpreted as a model for the sound energy propagation into an empty enclosure. Our goal is to obtain the outgoing sound radiance leaving a point on a surface $\mathbf{r}_{b}$ as a combination of the emitted radiance and the amount of the reflected radiance incoming from all visible surfaces.

The solution to this equation is composed by the solution to the homogeneous form and a particular solution. The general solution of the homogeneous first order partial differential equation is given by the following expression,

$$
L_{H}(\mathbf{r}, \hat{\mathbf{s}}, t)=e^{-m\left(\mathbf{r}-\mathbf{r}^{\prime}\right) \cdot \hat{\mathbf{s}}} \phi\left[t-\frac{\left(\mathbf{r}-\mathbf{r}^{\prime}\right) \cdot \hat{\mathbf{s}}}{c}\right],
$$

where $\phi[u]$ is an arbitrary function and $\mathbf{r}^{\prime}$ is a reference point. This equation follows the same expression as a plane wave that propagates in direction $\hat{\mathbf{s}}$ from $\mathbf{r}^{\prime}$ to $\mathbf{r}$ and is attenuated along its path by the air attenuation factor $m$ over distance $\left(\mathbf{r}-\mathbf{r}^{\prime}\right) \cdot \hat{\mathbf{s}}$. From Eq. (4.48) it can be seen that $\phi[u]$ also represents energy propagation. This function expresses the radiance at a delayed time $\left(\mathbf{r}-\mathbf{r}^{\prime}\right) \cdot \hat{\mathbf{s}} / c$. Therefore, since it is a plane wave, the radiance has to be evaluated at position $\mathbf{r}^{\prime}$,

$$
L_{H}(\mathbf{r}, \hat{\mathbf{s}}, t)=e^{-m\left(\mathbf{r}-\mathbf{r}^{\prime}\right) \cdot \hat{\mathbf{s}}} L\left(\mathbf{r}^{\prime}, \hat{\mathbf{s}}, t-\frac{\left(\mathbf{r}-\mathbf{r}^{\prime}\right) \cdot \hat{\mathbf{s}}}{c}\right) .
$$




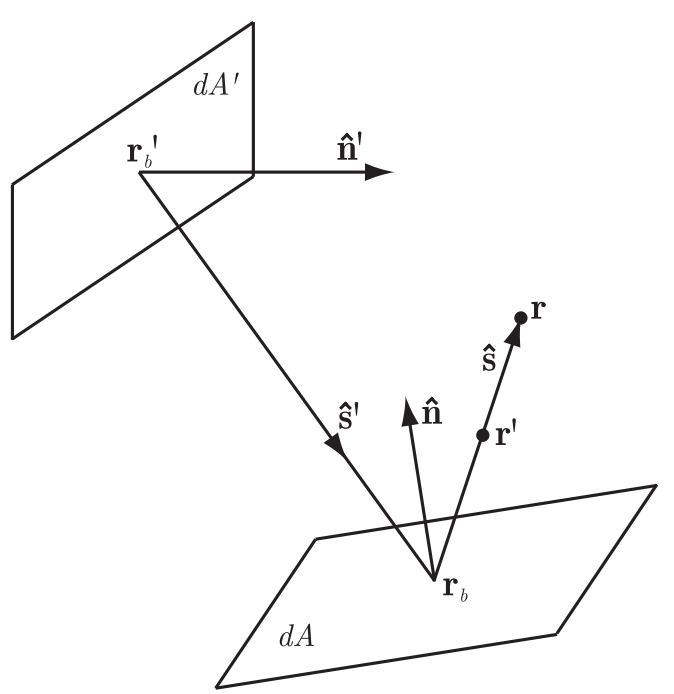

Figure 4.6. Distance between points $\boldsymbol{r}$ and $\boldsymbol{r}_{b}$.

Note that plane propagating waves are usually described in geometrical acoustics by rays representing the sound energy propagation in the direction of that plane wave.

To solve this Cauchy-problem subject to initial conditions let $\mathbf{r}^{\prime}$ lie on the boundary $\mathbf{r}_{b}$, then the acoustic radiative transfer boundary conditions provide an expression for $L\left(\mathbf{r}^{\prime}, \hat{\mathbf{s}}, t\right)$.

Substituting Eq. (4.18) into Eq. (4.49) yields

$$
\begin{array}{r}
L(\mathbf{r}, \hat{\mathbf{s}}, t)=e^{-m\left(\mathbf{r}-\mathbf{r}_{b}\right) \cdot \hat{\mathbf{s}}}\left[L_{0}\left(\mathbf{r}_{b}, \hat{\mathbf{s}}, t-\frac{\left(\mathbf{r}-\mathbf{r}_{b}\right) \cdot \hat{\mathbf{s}}}{c}\right)\right. \\
\left.+\int_{\Omega-} R_{F}\left(\mathbf{r}_{b} ; \hat{\mathbf{s}}^{\prime}, \hat{\mathbf{s}}\right) L\left(\mathbf{r}_{b}, \hat{\mathbf{s}}^{\prime}, t-\frac{\left(\mathbf{r}-\mathbf{r}_{b}\right) \cdot \hat{\mathbf{s}}}{c}\right)\left(\hat{\mathbf{s}}^{\prime} \cdot-\hat{\mathbf{n}}\right)\right] d \Omega^{\prime} .
\end{array}
$$

using the notation expressed in Fig. 4.6.

This equation is parameterized by direction as the integration term shows. In room acoustics the integration term is usually parameterized by position, changing the infinitesimal solid angle by an infinitesimal area surface along the incoming radiance from all visible boundary points $\mathbf{r}_{b}^{\prime}$. 
Moreover, sound radiance at these points $L\left(\mathbf{r}_{b}^{\prime}, \hat{\mathbf{s}}^{\prime}, t\right)$ should be defined from Eq. (4.49),

$$
\begin{array}{r}
L(\mathbf{r}, \hat{\mathbf{s}}, t)=e^{-m\left(\mathbf{r}-\mathbf{r}_{b}\right) \cdot \hat{\mathbf{s}}}\left[L_{0}\left(\mathbf{r}_{b}, \hat{\mathbf{s}}, t-\frac{\left(\mathbf{r}-\mathbf{r}_{b} \cdot \hat{\mathbf{s}}\right)}{c}\right)\right. \\
+\int_{\partial V} R_{F}\left(\mathbf{r}_{b} ; \hat{\mathbf{s}}^{\prime}, \hat{\mathbf{s}}\right) e^{-m\left(\mathbf{r}_{b}-\mathbf{r}_{b}^{\prime}\right) \cdot \hat{\mathbf{s}}^{\prime}} \\
\left.L\left(\mathbf{r}_{b}^{\prime}, \hat{\mathbf{s}}^{\prime}, t-\frac{\left(\mathbf{r}-\mathbf{r}_{b}\right) \cdot \hat{\mathbf{s}}+\left(\mathbf{r}_{b}-\mathbf{r}_{b}^{\prime}\right) \cdot \hat{\mathbf{s}}^{\prime}}{c}\right) g\left(\mathbf{r}_{b}, \mathbf{r}_{b}^{\prime}\right) d A^{\prime}\right],
\end{array}
$$

where $g\left(\mathbf{r}_{b}, \mathbf{r}_{b}^{\prime}\right)$ is the geometrical term defined as follows,

$$
\begin{gathered}
d \Omega^{\prime}=d A^{\prime} \frac{\left(\hat{\mathbf{s}}^{\prime} \cdot \hat{\mathbf{n}}^{\prime}\right)}{\left(\left(\mathbf{r}_{b}-\mathbf{r}_{b}^{\prime}\right) \cdot \hat{\mathbf{s}}^{\prime}\right)^{2}}, \\
g\left(\mathbf{r}_{b}, \mathbf{r}_{b}^{\prime}\right)=\frac{\left(\hat{\mathbf{s}}^{\prime} \cdot \hat{\mathbf{n}}^{\prime}\right)\left(\hat{\mathbf{s}}^{\prime} \cdot-\hat{\mathbf{n}}\right)}{\left(\left(\mathbf{r}_{b}-\mathbf{r}_{b}^{\prime}\right) \cdot \hat{\mathbf{s}}^{\prime}\right)^{2}} .
\end{gathered}
$$

Usually, an artificial visibility term $v\left(\mathbf{r}_{b}, \mathbf{r}_{b}^{\prime}\right)$ is incorporated in the equation to ensure that boundary points are reachable and there are no obstacles between them.

Here the source term $L_{0}\left(\mathbf{r}_{b}, \hat{\mathbf{s}}, t\right)$ is the sound emittance. A boundary surface can be considered as an emitter in the modelling of such processes as sound energy transmission through a room boundary or the primary reflected radiance of a point source $q(\mathbf{r}, \hat{\mathbf{s}}, t)$ with a direct contribution coming from inside of the room, not from another boundary position.

In order to obtain a complete solution, the direct sound contribution from a point source in the room should be incorporated in Eq. (4.52). This can be done through the particular solution defined by

$$
L_{P}(\mathbf{r}, \hat{\mathbf{s}}, t)=\int_{-\infty}^{t} q\left(\mathbf{r}_{s}, \hat{\mathbf{s}}, \tau\right) G\left(\mathbf{r}-\mathbf{r}_{s}, \hat{\mathbf{s}}, t-\tau\right) d \tau,
$$

where $\mathbf{r}_{s}$ is the source position and $G(\mathbf{r}, \hat{\mathbf{s}}, t)$ is the free-field Green's function associated with Eq. (4.47). The free-field Green's function of acoustic radiative transfer equation is

$$
G(\mathbf{r}, \hat{\mathbf{s}}, t)=\frac{e^{-m(\mathbf{r} \cdot \hat{\mathbf{s}})}}{4 \pi(\mathbf{r} \cdot \hat{\mathbf{s}})^{2}} \delta\left(t-\frac{\mathbf{r} \cdot \hat{\mathbf{s}}}{c}\right),
$$


where $\delta(t)$ is the dirac delta function. This leads to

$$
L_{P}(\mathbf{r}, \hat{\mathbf{s}}, t)=\frac{e^{-m\left(\mathbf{r}-\mathbf{r}_{s}\right) \cdot \hat{\mathbf{s}}}}{4 \pi\left(\left(\mathbf{r}-\mathbf{r}_{s}\right) \cdot \hat{\mathbf{s}}\right)^{2}} q\left(\mathbf{r}_{s}, \hat{\mathbf{s}}, t-\frac{\left(\mathbf{r}-\mathbf{r}_{s}\right) \cdot \hat{\mathbf{s}}}{c}\right)
$$

The last equation represents the direct sound energy from a point source inside the room that arrives after some time with a spherical divergence and attenuation due to air absorption. The combination of Eq. (4.52) and Eq. (4.57) is a solution that makes it possible to calculate the sound radiance at any point in space propagating at $\hat{\mathbf{s}}$ direction as a result of the sum of the direct and reflected sound energy.

The original room-acoustic rendering equation incorporating a timedependence parameter is a particularization of Eq. (4.52) when a boundary point $\mathbf{r}_{b}$ is calculated. Although this derivation confirms the room-acoustic rendering equation proposal of Ref. [Siltanen et al., 2007a], it should be noted that the approach of this manuscript extends details about the implementation of this equation, such as time delays and direct sound. The reflection kernel term in Eq. (4.46) becomes

$$
R\left(\mathbf{r}_{b} ; \hat{\mathbf{s}}^{\prime}, \hat{\mathbf{s}}\right)=e^{-m\left(\mathbf{r}_{b}-\mathbf{r}_{b}^{\prime}\right) \cdot \hat{\mathbf{s}}^{\prime}} R_{F}\left(\mathbf{r}_{b} ; \hat{\mathbf{s}}^{\prime}, \hat{\mathbf{s}}\right) g\left(\mathbf{r}_{b}, \mathbf{r}_{b}^{\prime}\right) v\left(\mathbf{r}_{b}, \mathbf{r}_{b}^{\prime}\right) .
$$

Since the room-acoustic rendering equation models only the energy exchange between surface points, the sound radiance arriving from all visible surfaces and the direct energy arriving from a point source need to be evaluated separately in order to obtain the total sound radiance at a receiver point within the room. However, with the Eq. (4.52), it can be obtained directly.

After this derivation, the room acoustic rendering equation can be considered a particularization of the acoustic radiative transfer equation for empty enclosures. 


\subsection{Derivation of acoustical radiosity}

In this section, the acoustical radiosity model is presented previously to its derivation from the acoustic radiative transfer model.

\subsubsection{Technical and mathematical foundations of acoustical radiosity}

The acoustical radiosity method reviewed in Sec. 3.4.3 uses an integral equation to describe a time-dependent radiation energy balance within an enclosure that contains $N$ discrete surfaces. The sound radiance is defined using the position $\mathbf{r}_{b}$ as a point on a surface. Then, the radiosity $B\left(\mathbf{r}_{b}, t\right)$ is described on a surface as energy intensity, or radiance leaving a surface element $\left(W m^{-2}\right)$.

$$
B\left(\mathbf{r}_{b}, t\right)=\int_{\Omega^{+}} L\left(\mathbf{r}_{b}, t\right)(\hat{s} \cdot \hat{n}) d \Omega
$$

where the integral is evaluated on a hemisphere $\Omega^{+}$. For incoming radiance this quantity is called irradiance, used in the integral equation method [Kuttruff, 4th edition, 2000, 1971, 1976].

A general form of the radiosity equation [Nosal et al., 2004a]

$$
B\left(\mathbf{r}_{\mathbf{b}}, t\right)=B_{0}\left(\mathbf{r}_{\mathbf{b}}, t\right)+R\left(\mathbf{r}_{\mathbf{b}}\right) \int_{\partial V} B\left(\mathbf{r}_{b}^{\prime}, t-\frac{\left(\mathbf{r}_{b}-\mathbf{r}_{b}^{\prime}\right)}{c}\right) d A^{\prime}
$$

It can be observed that the latter Eq. (4.60) follows the same structure than Eq. (4.46) where $B_{0}\left(\mathbf{r}_{\mathbf{b}}, t\right)$ is the time-dependent direct contribution radiosity and $B\left(\mathbf{r}_{b}^{\prime}, t-\frac{\left(\mathbf{r}_{b}-\mathbf{r}_{b}^{\prime}\right)}{c}\right)$ is the amount of the reflected time-dependent radiosity due to all visible surfaces.

In the acoustical radiosity method, a common simplification is made ignoring the directional dependence of the radiance and assuming a Lambert's law diffuse reflectance and emittance for all surfaces, thus in Eq. (4.60), the reflection kernel $R\left(\mathbf{r}_{b} ; \hat{\mathbf{s}}^{\prime}, \hat{\mathbf{s}}\right)$ has lost its directional dependence $R\left(\mathbf{r}_{\mathbf{b}}\right)$. 


\subsubsection{Relation to the acoustic radiative transfer model}

The radiosity method can be derived from Eq. (4.52) by using the diffuse reflectance factor

$$
R_{F}\left(\mathbf{r}_{b} ; \hat{\mathbf{s}}^{\prime}, \hat{\mathbf{s}}\right)=R_{F}\left(\mathbf{r}_{b}\right) / \pi,
$$

where $R_{F}\left(\mathbf{r}_{b}\right)$ is the reflection coefficient of the material at point $\mathbf{r}_{b}$. This simplifies the room-acoustic rendering equation by omitting the directional dependence.

\subsection{Discussion about assumptions, advantages and disadvantages}

The basic assumptions of the acoustic radiative transfer model (see Sec. 4.4.1) are the same as those of geometrical acoustics where the wavelengths are regarded as short compared with the room dimensions and interference between rays is neglected [Kuttruff, 1997]. Since no phase relations are taken into account, it is important to note that the Schroeder's frequency limit (Eq. (2.64)) must be satisfied [Schroeder, 1962]. This restriction limits the validity of the acoustic radiative transfer equation to medium and high frequencies.

The main contribution of the acoustic radiative transfer model is that expands classical geometrical room acoustics modelling algorithms incorporating a propagation medium that can scatter (Eq. (4.5)) and absorb energy (Eq. (4.6)).

Moreover, the model can handle complex sound sources and arbitrary reflections both on boundaries and in the medium. The scattering on the boundaries uses the reflection function (see Sec. 4.4.3) that represents the directional distribution of the reflected energy for each incoming angle. In the case of the scattering medium, the phase function is equivalent to the reflection function (Eq. (4.14)). 
Absorption and scattering of the medium are important in large rooms and fitted rooms [Kuttruff, 4th edition, 2000]; accordingly this model can predict realistic environments where modes and interference effects are not essential for our perception of sound. The scattering medium approximation could be useful in rooms where the interior contains noticeable objects (fittings), e.g. machines, chairs, and desks. Some factories, classrooms, and offices are studied as fitted rooms [Kurze, 1985]. In these cases, the theory for empty rooms no longer holds and the fittings inside the room should be considered. Although these properties of the medium are defined with statistical terms that simplify the model because air conditions in rooms are usually isotropic, it is possible to expand the model to spatially dependent medium properties. It is important to remember that the absorption and scattering coefficient are frequency dependent; therefore sound radiance propagation may be separated into different frequency sub-bands, which implies that a calculation must be carried for each frequency band. Although in this thesis a room acoustics simulation model has been developed, it should be emphasized that it is used in this first approach more as a theoretical model than a simulation model in order to establish and unify the principles of a number of room acoustics simulation models.

The radiative transfer equation is a specialization of a generic transport equation that describes transport phenomena such as heat transfer, mass transfer and fluid dynamics. The transport equation for solving the sound propagation problem in specific environments was also previously studied in technical literature. In environmental acoustics, the application of the the transport theory for simulating street with small width was shown [Pollès et al., 2004, 2005] adapting it to predict the sound propagation in urban areas. In room acoustics, to model sound energy propagation in long spaces an 1-D transport approximation of a 3-D transport equation was derived [Jing et al., 2010, Jing and Xiang, 2010] that follows a similar structure than the radiative transfer equation (see Eq. (4.16)). In this thesis, the acoustic radiative transfer model has been proposed as a theoretical framework to cover all the room modelling techniques based on geometrical acoustics, 
especially to set the foundations of the acoustic diffusion equation model.

The high computational cost involved in the solution of the integrodifferential equation in five independent variables is one of its limitations [Siegel and Howell, 1981]. Nevertheless, analytical solutions to the radiative transfer equation exist for simple cases, but for media with complex multiple scattering effects numerical methods are required [Rohsenow et al., 1998]. Although the problem could be simulated by numerical Monte Carlo algorithm [Howell, 1998, Iwabuchi, 2006] and other numerical methods [Kanschat, 2008], the choice was done to find simplified solutions. In order to evaluate the possibilities of this model in predicting sound fields in rooms, two particularisations have been examined leading to an analytical approximation, the acoustic diffusion model, and a simplified integral solution, the rendering equation model, both corresponding to previously published well-know methods. In addition to providing a common and proper foundation, this model links the acoustic diffusion equation model with geometrical acoustics models deriving each method with the same theoretical foundations and clarifying theirs assumptions and limitations. Compared to the diffusion equation model, the acoustic radiative transfer model adds the possibility of modelling non-diffuse reflections, and compared to the roomacoustic rendering equation, the acoustic radiative transfer model adds the possibility of modelling a scattering medium.

The diffusion approximation needs particular assumptions in addition to the general assumptions mentioned above. First, it requires the assumption of directional broadening (Sec. 4.5.2.1), which means that the scattering density must be high and the reflection of energy must dominate over absorption. To achieve this condition, all boundaries are assumed totally diffusely reflecting, leading to an isotropic phase function in the radiative transfer equation (Eq. (4.36)). Accordingly, the receptor must also be sufficiently far from the source and the boundaries in space and in time to secure that enough reflection events have occurred when they reach the receptor, making the acoustic diffusion model inherently not valid for the direct field, 
only for the reverberant field. However, the accuracy near the boundaries is improved with the boundary conditions exposed in Sec. 4.5.2.2. Secondly, the assumption of temporal broadening (Sec. 4.5.2.1) must be fulfilled, which means that the sound energy flow vector and the sound energy density may be nearly isotropic after sufficient scattering, and hence their variation per mean free path must be low and the expression for the sound energy flow vector is analogous to Fick's law (Eq. (4.19)). Therefore, the directional meaning of these variables is lost. The source must be also isotropic, implying that only omnidirectional sound sources $\mathbf{q}_{1}(\mathbf{r}, \hat{\mathbf{s}}, t)=0$ are applicable.

Theoretically, the acoustic diffusion model is mainly valid for predicting the late part of decay curves for low absorption cases in rooms. The diffusion equation model can be seen as a geometrical acoustics method, similar to ray-tracing and image-source models. Studies in the technical literature have shown that the diffusion model is computationally efficient compared with ray-tracing model [Valeau et al., 2006, Xiang et al., 2009]. The diffusion model also provides more satisfactory results than statistical room acoustics theory since it is capable of modelling the non-uniformity of sound fields [Picaut et al., 1999b, Valeau et al., 2006]. Note that anisotropic component of the radiance is used in the approximation (Sec. 4.5.2.1).

The room-acoustic rendering equation has been shown to be applicable to predict room sound fields in realistic environment with reasonable results in a round robin test [Siltanen et al., 2007a]. Moreover, based on this general equation, an acoustic radiance transfer method, both for diffuse and non-diffuse reflection, has been presented [Siltanen et al., 2007b] and have been extended to model diffraction [Siltanen and Lokki, 2008]. Its main limitation is that the scattering events in the medium are neglected (see Eq. 4.47). Scattering medium might be modelled as individual objects with little geometry with their own scattering and absorption coefficients, which could be computationally more demanding than using the statistical scattering medium model. Its prediction results are expected to be more 
accurate than the acoustic diffusion model values, because this equation is not an approximation, especially when the specular reflections are dominant, with high absorption cases or when the assumptions of the directional and temporal broadening assumptions are not met. However, this precision is achieved with higher computational costs.

\subsection{Summary}

In this chapter, an acoustic radiative transfer equation theoretical approach for modelling acoustic energy propagation is presented . It relies on geometrical acoustics. In relation with this thesis, the acoustic radiative transfer model main contribution is that it allows to clarify the foundations of the acoustic diffusion equation model and also find a theoretical connection between the diffusion equation model and geometrical models. In Fig. 4.7 a proposed diagram of room acoustics modelling techniques after the presented acoustic radiative transfer model is shown.

In order to evaluate the possibilities of this model for predicting sound fields in rooms, two of the most relevant equation models that have recently appeared in the architectural acoustics field, the diffusion equation model and the room-acoustic rendering equation, are derived. Thus, the acoustic radiative transfer model provides a common geometrical acoustics framework for both models. It also establishes a direct link between them. Moreover, this model makes it possible to include the diffusion equation model in the geometrical acoustics methods' group.

Using the proposed propagation model, the theoretical advantages and limitations of the two methods are enunciated. It is suggested that the acoustic diffusion model is mainly valid for predicting the late reverberation part of decay curves in rooms with low absorption. On the other hand, it is suggested that the room-acoustic rendering equation cannot inherently handle scattering events in the medium. However, the room-acoustic rendering equation can model scattering by individual objects with some geometry as 


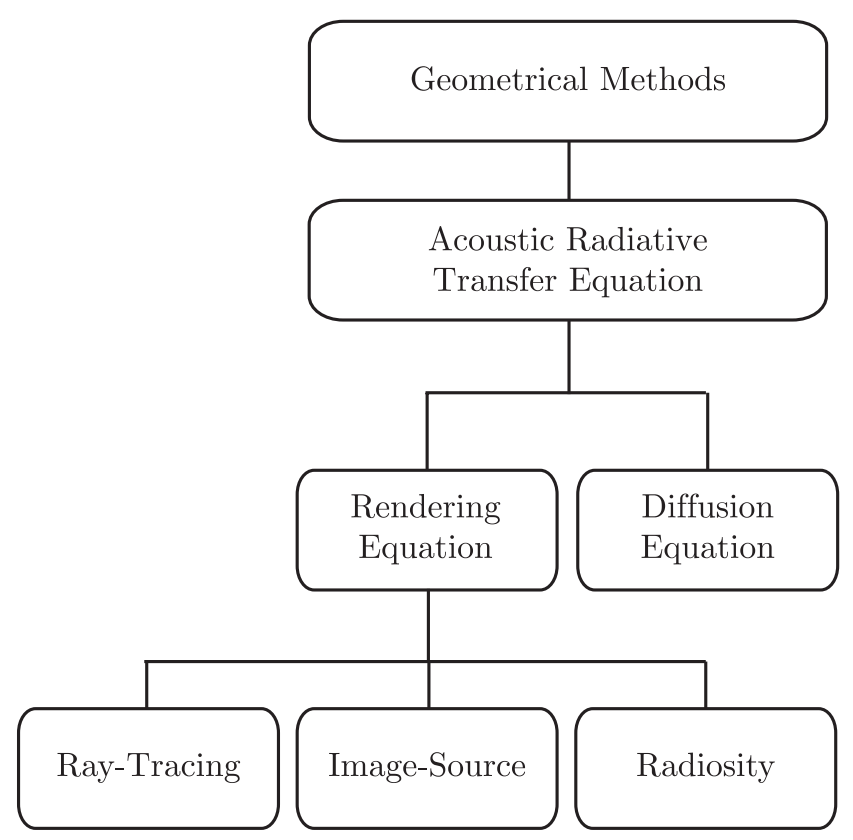

Figure 4.7. Proposed diagram of different approaches to room acoustics modelling after the acoustic radiative transfer model.

surfaces within the room.

In the following chapter (Chap. 5), an investigation for providing a suitable finite-difference scheme to implement the acoustic diffusion equation model is carried out. 


\section{Implementation of a Diffusion Equation Model for Sound Field Modelling} by a Finite-Difference Scheme

As PRESENTED IN PREVIOUs CHAPTER, the acoustic diffusion equation model is an asymptotic approximation to the radiative transfer equation model. The resulting system of equations are called partial differential equations. Finite-difference techniques are widely used in wave-based method (see Sec. 3.3.2.1). In this chapter, an investigation for providing a suitable finite-difference scheme to implement the acoustic diffusion equation model is carried out ${ }^{1}$.

\subsection{Introduction}

As described in Sec. 3.4.5 a diffusion equation model to predict roomacoustic parameters, such as the reverberation time and the sound pressure level, has been successfully applied in different room scenarios. In this

\footnotetext{
${ }^{1}$ Portions of this chapter constitute the major part of a paper accepted (in-press) by the Applied Acoustics Journal [Navarro et al., in-press, 2012].
} 
chapter, the implementation of this acoustic diffusion equation model using finite-difference schemes is investigated, and the appropriate scheme is chosen.

The acoustic diffusion equation models the sound field in enclosures with diffusely reflecting boundaries with sound particles with the same constant energy propagating along straight lines. This model, which takes into account the total diffuse reflections, has been suggested to be mainly valid for predicting the late part of the sound fields [Valeau et al., 2006, Xiang et al., 2009] and in Sec. 4.8. In previous work, a finite element method (FEM) commercial software has usually been applied to model a wide variety of room types [Valeau et al., 2006, 2007, Xiang et al., 2009, Billon et al., 2008a]. Here, the finite-difference methods (FDM) are presented as an alternative numerical technique that can be easily implemented. A discussion about the general pros and cons of applying FEM or FDM in presented in Sec. 3.5.3. In summary, in comparison to FDM, element methods have an advantage of grid adaptability to irregular boundaries, which can be achieved by the use of non-uniform shapes. However, this approach increases the computational cost. Straightforward algorithms in FDM schemes are computationally more efficient. Furthermore, using numerical grid generation approach [Thompson et al., 1999] FDM schemes can deal with irregular geometries, while still maintaining theirs simplicity. Consequently, in this thesis, the finite difference methods are adopted for further research of the acoustic diffusion equation model into room acoustics modelling.

This chapter reviews the possible finite-difference schemes available in the literature to find the most suitable scheme to implement a numerical solution for the diffusion equation model. Special attention is paid to the inclusion of the air absorption effect into the scheme and the analysis of such effect on the overall stability condition. Then, two selected explicit schemes, forward-time centred-space (FTCS) and Dufort-Frankel (DF), are compared with each other. These schemes are evaluated in several cubic 
rooms with different absorption distributions, and the predicted values are compared with the data obtained from Barron's statistical theory and two geometrical models: radiosity and diffuse ray-tracing. Thus, this chapter aims to evaluate the accuracy, the stability and the computational cost of a finite-difference implementation of a diffusion equation model.

This chapter is organised as follows. In Sec. 5.2, several available finitedifference schemes for the acoustic diffusion model are investigated, and theirs stability conditions are derived with the air absorption included in the equation. Then, the implementation of the algorithm is evaluated with the chosen schemes in Sec. 5.3. Finally, the conclusions are presented.

\subsection{Finite-difference schemes for the acoustic dif- fusion model}

In this section, the implementation of this acoustic diffusion equation model, both the governing and the boundary condition equations (see Sec. 4.5) by means of finite-difference schemes is thoughtfully investigated and a justified selection of the appropriate scheme is done. Furthermore, special attention is paid to the inclusion of air absorption into the scheme and to analyse how this could affect the overall stability.

The finite-difference method is a numerical technique used for solving a differential equation over a given region subject to the specified boundary conditions, which are based on a finite-difference approach of the involved derivatives of a partial differential equation. E. g., the first derivative may be approached by using

$$
\begin{aligned}
\frac{\partial y(x, t)}{\partial t} & \simeq \frac{y(x, t+\Delta t)-y(x, t)}{\Delta t} \\
& \simeq \frac{y(x, t)-y(x, t-\Delta t)}{\Delta t},
\end{aligned}
$$


When the finite-difference approach is used, the problem domain is discretised so that the values of the unknown dependent variable are considered only at a finite number of nodal points or cells instead of at every point over the region. A discretised function is defined as follows,

$$
w(\mathbf{r}, t)=w(i \Delta x, j \Delta y, k \Delta z, n \Delta t)=w_{i, j, k}^{n},
$$

where the temporal index $n$, and the spatial indexes $i, j$ and $k$ have been introduced, together with the temporal discretisation $\Delta t$ and the spatial discretisation in the cartesian axis $\Delta x, \Delta y$ and $\Delta z$. Latters are defined as the inverse of the temporal and spatial resolutions respectively. For example, low resolution implies large cell sizes and high resolution implies small cell sizes.

\subsubsection{Forward-time centred-space scheme}

\subsubsection{Governing equation discretisation}

A straightforward finite-difference approach to solve the acoustic diffusion equation that includes the atmospheric absorption factor is the forwardtime centred-space scheme (FTCS) [Strikwerda, 1989]. This scheme makes use of a central difference for the second derivative in space and a forward difference for the first derivative in time [Navarro et al., 2010a]. The goal is to determine a system of algebraic equations which solution tends to the analytical solution. Here, the mathematical developments are done in a 3-D space. Applying this scheme over the derivatives of Eq. (4.23), it yields

$$
\begin{aligned}
\frac{w_{i, j, k}^{n+1}-w_{i, j, k}^{n}}{\Delta t} & =D\left[\frac{w_{i+1, j, k}^{n}-2 w_{i, j, k}^{n}+w_{i-1, j, k}^{n}}{\Delta x^{2}}\right. \\
& +\frac{w_{i, j+1, k}^{n}-2 w_{i, j, k}^{n}+w_{i, j-1, k}^{n}}{\Delta y^{2}} \\
& \left.+\frac{w_{i, j, k+1}^{n}-2 w_{i, j, k}^{n}+w_{i, j, k-1}^{n}}{\Delta z^{2}}\right] \\
& -c m w_{i, j, k}^{n} .
\end{aligned}
$$


Finally, the finite-difference equation may be rearranged

$$
\begin{aligned}
w_{i, j, k}^{n+1} & =\left[1-\frac{2 D \Delta t}{\Delta x^{2}}-\frac{2 D \Delta t}{\Delta y^{2}}-\frac{2 D \Delta t}{\Delta z^{2}}\right] w_{i, j, k}^{n} \\
& +\frac{D \Delta t}{\Delta x^{2}}\left[w_{i+1, j, k}^{n}+w_{i-1, j, k}^{n}\right] \\
& +\frac{D \Delta t}{\Delta y^{2}}\left[w_{i, j+1, k}^{n}+w_{i, j-1, k}^{n}\right] \\
& +\frac{D \Delta t}{\Delta z^{2}}\left[w_{i, j, k+1}^{n}+w_{i, j, k-1}^{n}\right]-c m w_{i, j, k}^{n} \Delta t .
\end{aligned}
$$

The source term, included as a soft source, is added at the suitable position as $w_{i_{s}, j_{s}, k_{s}}^{n+1}=w_{i_{s}, j_{s}, k_{s}}^{\prime n+1}+2 \Delta t P_{i_{s}, j_{s}, k_{s}}^{n}$ where $w_{i_{s}, j_{s}, k_{s}}^{n+1}$ is the value after the cells were updated using Eq. (5.5).

FTCS is called the simple explicit form of finite-difference approximation of the diffusion equation because it involves only one unknown at each time level, which can be directly calculated when the neighbour values at the previous time level are available. The accuracy of this scheme is firstorder in time and second-order in space. The error order is defined as $O[(\Delta t)]+O\left[(\Delta \nu)^{2}\right]$, where $\nu=[x, y, z]$ and $O[\cdot]$ is the remaining error associated with a specific finite-difference, and the variable inside the brackets determines the upper boundary on the growth rate of that scheme.

\subsubsection{Boundary conditions discretisation}

Eq. (5.5) is called explicit form because it involves only one unknown $w_{i, j, k}^{n+1}$. Clearly, the system of equations obtained from Eq. (5.5), i.e. in dimension $x$, for $i=1,2, \ldots, L_{x-1}$, provides $L_{x-1}$ algebraic relations but contains $L_{x+1}$ unknown nodes at time step $n+1,\left(i=0,1,2, \ldots, L_{x}\right)$. Additional relations are needed to make the number of equations equal to the number of unknown variables. These variables are obtained from boundary conditions, as explained as follows.

For simplicity, let us adopt a boundary surfaces oriented on $x$-axis at 
both positions $x=0$ and $x=l_{x}$

$$
\begin{aligned}
-D \frac{\partial w(x, t)}{\partial x}+c A_{x}(x) w(x, t) & =0 \text { at } x=0, \\
D \frac{\partial w(x, t)}{\partial x}+c A_{x}(x) w(x, t) & =0 \text { at } x=l_{x},
\end{aligned}
$$

where the acoustical density energy at the boundary nodes $i=0$ and $i=$ $L_{x}$ are unknown. Additional relations by discretising these two boundary conditions are obtained.

A very simple approach to discretise these boundary conditions is to use the forward finite-difference scheme for Eq. (5.6) and the backward finite-difference scheme for Eq. (5.7)

$$
\begin{aligned}
D \frac{w_{0, j, k}^{n}-w_{1, j, k}^{n}}{\Delta x} & =-c A_{x_{0, j, k}} w_{0, j, k}^{n}, \\
D \frac{w_{L_{x}, j, k}^{n}-w_{L_{x-1}, j, k}^{n}}{\Delta x} & =-c A_{x_{L_{x}, j, k}} w_{L_{x}, j, k}^{n} .
\end{aligned}
$$

Eq. (5.8) and Eq. (5.9) are arranged as

$$
\begin{aligned}
w_{0, j, k}^{n} & =\frac{w_{1, j, k}^{n}}{\left(1+\frac{c A_{x_{0, j, k}} \Delta x}{D}\right)}, \\
w_{L_{x}, j, k}^{n} & =\frac{w_{L_{x-1}, j, k}^{n}}{\left(1+\frac{c A_{x_{L x}, j, k} \Delta x}{D}\right)},
\end{aligned}
$$

but the results with this discretisation are only first-order accuracy $O[(\Delta x)]$.

To obtain a complete numerical solution, with the same truncation error as Eq. (5.5), a second-order accuracy $O\left[(\Delta x)^{2}\right]$ is possible if central finite-difference schemes to discretise the first derivatives in these boundary conditions are used [Necati, 1994]. Therefore, let us consider fictitious nodes $i=-1$ and $i=L_{x+1}$ with their corresponding acoustic energy density $w_{-1, j, k}^{n}$ and $w_{L_{x+1}, j, k}^{n}$ respectively. These new values are necessary in order to apply a central finite-difference scheme. These fictitious nodes are obtained by extending the region by $\Delta x$ both sides, as illustrated in Fig. 5.1. 
Applying a central finite-difference scheme to discretise the boundary conditions along with these fictitious nodes, next relations are obtained

$$
\begin{aligned}
D \frac{w_{-1, j, k}^{n}-w_{1, j, k}^{n}}{2 \Delta x} & =-c A_{x_{0, j, k}} w_{0, j, k}^{n}, \\
D \frac{w_{L_{x+1}, j, k}^{n}-w_{L_{x-1}, j, k}^{n}}{2 \Delta x} & =-c A_{x_{L_{x}, j, k}} w_{L_{x}, j, k}^{n} .
\end{aligned}
$$

Two additional relations needed to eliminate these fictitious energy densities are determined by evaluating Eq. (5.5) at positions $i=0$ and $i=M_{x}$,

$$
\begin{aligned}
w_{0, j, k}^{n+1} & =r w_{-1, j, k}^{n}+(1-2 r) w_{0, j, k}^{n}+r w_{1, j, k}^{n}, \\
w_{L_{x}, j, k}^{n+1} & =r w_{L_{x-1}, j, k}^{n}+(1-2 r) w_{L_{x}, j, k}^{n}+r w_{L_{x+1}, j, k}^{n},
\end{aligned}
$$

where $r=\frac{D \Delta t}{(\Delta x)^{2}}$ and the air absorption is neglected.

Then, the following two expressions are obtained eliminating $w_{-1, j, k}^{n}$ and $w_{L_{x+1}, j, k}^{n}$ when Eq. (5.12) and Eq. (5.13) are combined:

$$
\begin{aligned}
w_{0, j, k}^{n+1} & =\left(1-2 r \beta_{0, j, k}\right) w_{0, j, k}^{n}+2 r w_{1, j, k}^{n}, \\
w_{L_{x}, j, k}^{n+1} & =\left(1-2 r \beta_{L_{x}, j, k}\right) w_{L_{x}, j, k}^{n}+2 r w_{L_{x-1}, j, k}^{n},
\end{aligned}
$$

being

$$
\begin{aligned}
\beta_{0, j, k} & =1+\frac{c A_{x_{0, j, k}} \Delta x}{D} \\
\beta_{L_{x}, j, k} & =1+\frac{c A_{x_{L_{x}, j, k}} \Delta x}{D} .
\end{aligned}
$$

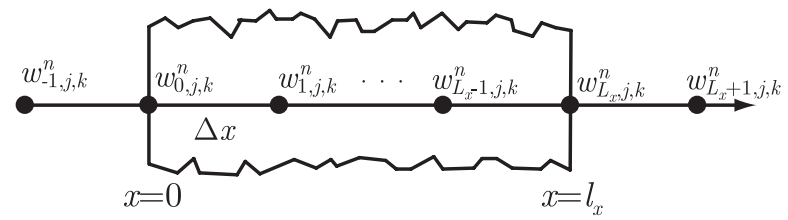

Figure 5.1. Fictitious nodes -1 and $L_{x+1}$ at fictitious $w_{-1, j, k}^{n}$ and $w_{L_{x+1}, j, k}^{n}$ 
Thus, Eq. (5.5) together with Eq. (5.14) are the complete finite-difference approximation of the acoustic diffusion equation, including air absorption, subjected to mixed boundary conditions.

\subsubsection{Von Neumman analysis}

In this section a Von Neumann analysis of the Eq. (5.5) is performed in order to establish its stability condition. This analysis consists on assuming $w(\mathbf{x}, t)=\xi^{t / \Delta t} e^{-\iota \mathbf{k}_{0}^{T} \mathbf{x}}$. It is defined $\iota=\sqrt{-1}, \xi$ is the amplification parameter, $T$ means the transposed vector and $\mathbf{k}_{0}=\left[k_{0_{x}}, k_{0_{y}}, k_{0_{z}}\right]$ is the wave number vector. Thus, this derivation aims to find under which conditions the requisite $\|\xi\| \leq 1$ (or alternatively, $\|\xi\|^{2} \leq 1$ ) is verified [Strikwerda, 1989].

In the discrete analysis, let us define $w_{i, j, k}^{n}=\xi^{n} e^{-\iota\left(k_{0 x} i \Delta x+k_{0_{y}} j \Delta y+k_{0_{z}} k \Delta z\right)}$. Once this expression is substituted into Eq. (5.5) it leads to,

$$
\begin{aligned}
\xi^{n+1}=[1 & \left.-\sum_{\nu=[x, y, x]}\left(\frac{2 D \Delta t}{\Delta \nu^{2}}\right)-c m \Delta t\right] \xi^{n} \\
& +\frac{D \Delta t}{\Delta x^{2}} \xi^{n}\left[e^{\iota k_{0} \Delta x}+e^{-\iota k_{0 x} \Delta x}\right] \\
& +\frac{D \Delta t}{\Delta y^{2}} \xi^{n}\left[e^{\iota k_{0_{y}} \Delta y}+e^{-\iota k_{0_{y}} \Delta y}\right] \\
& +\frac{D \Delta t}{\Delta z^{2}} \xi^{n}\left[e^{\iota k_{0_{z}} \Delta z}+e^{-\iota k_{0_{z}} \Delta z}\right]
\end{aligned}
$$

After some simplification, it yields,

$$
\xi=1+\sum_{\nu=[x, y, x]} \frac{2 D \Delta t}{\Delta \nu^{2}}\left(\cos \left(\theta_{\nu}\right)-1\right)-c m \Delta t,
$$

where $\theta_{\nu}=k_{0_{\nu}} \Delta \nu$. Therefore, in order to accomplish with the stability criterion, the following relations must be satisfied:

$$
-1 \leq 1-\sum_{\nu=[x, y, x]} \frac{4 D \Delta t}{\Delta \nu^{2}} \sin ^{2}\left(\theta_{\nu} / 2\right)+c m \Delta t \leq 1 .
$$

The second inequality is unconditionally true, since $D \Delta t / \Delta \nu^{2}$ and $m$ are strictly positive values. However, the first inequality is conditionally 
true and it has to be calculated under which conditions it occurs. After some simple algebraic operations, this inequality becomes

$$
\sum_{\nu=[x, y, x]} \frac{4 D \Delta t}{\Delta \nu^{2}} \sin ^{2}\left(\theta_{\nu} / 2\right)-c m \Delta t \leq 2 .
$$

The left side of the inequality reach its maximum value when $\sin ^{2}\left(\theta_{\nu} / 2\right)=$ 1 , giving

$$
\sum_{\nu=[x, y, x]} \frac{4 D \Delta t}{\Delta \nu^{2}}-c m \Delta t \leq 2 .
$$

Rearranging this former expression, it leads to the stability condition

$$
\Delta t\left[D\left(\frac{1}{(\Delta x)^{2}}+\frac{1}{(\Delta y)^{2}}+\frac{1}{(\Delta z)^{2}}\right)-\frac{c m}{4}\right] \leq \frac{1}{2}
$$

When the stability criterion is violated, the solution begins to oscillate and diverge, while the results obtained with right values are stable and in good agreement with the exact solution for the problem.

If the solution of that finite-difference scheme should remain stable (i.e., non-divergent or non-oscillatory), the value of $\Delta t$ should be restricted to

$$
\Delta t \leq \frac{1}{2} \frac{1}{\left[\sum_{\nu=[x, y, z]} \frac{1}{(\Delta \nu)^{2}}-\frac{c m}{4}\right]} .
$$

This stability criterion implies that for a given $\Delta \nu$, the magnitude of $\Delta t$ cannot exceed the limit imposed by Eq. (5.22). It should be noted that compared to having no atmospheric attenuation effect, the stability condition is slightly more permissive, allowing the use of higher time discretisation, i.e., a lower temporal resolution. Nevertheless, the air absorption $m$ usually varies from $10^{-5}$ to $0.05 \mathrm{~m}^{-1}$, corresponding to a frequency range of $50-16,000 \mathrm{~Hz}$ for an acoustic wave propagating in air at $20{ }^{\circ} \mathrm{C}$ and a $50 \%$ relative humidity. Under these conditions, the FTCS algorithm stability is not too dependent on the air absorption and may be considered negligible. 
Therefore, assuming that $\Delta \nu=\Delta x=\Delta y=\Delta z$ the stability condition without the air absorption becomes,

$$
\Delta t \leq \frac{(\Delta \nu)^{2}}{6 D}
$$

\subsubsection{Suitability}

From the observation of Eq. (5.5), some features of the FTCS diffusion equation implementation are derived: firstly, it is only necessary to store the discrete acoustic energy density at one instant $n$. Secondly, the stability condition shows that FTCS scheme is dissipative of order 2 as long as Eq. (5.22) is strictly assumed as an equality. Dissipation is a desirable property for finite-difference schemes for parabolic PDEs to have, since then the numerical solution becomes smoother in time, as does the analytical solution [Strikwerda, 1989]. For that reason, a practical consideration is to substitute the term $1 / 2$ of Eq. (5.22) by 0.49. Moreover, it should be emphasised that the appearance of the variable $D$ in the stability criterion in Eq. (5.22) introduces a direct dependence with the entire analysed volume, which means that for a given $\Delta t$, a larger room requires a greater $\Delta \nu$ size, and a lower number of cells have to be calculated. Table 5.1 shows a set of examples of the minimum values of $\Delta \nu$ for a given sampling frequency $f_{s}$ in 3-D cubic rooms and assuming the same step size in all the directions, with $c=340 \mathrm{~m} / \mathrm{s}$. Those values show how $\Delta \nu$ increase its size when the room volume increases as well.

Although it may seems beneficial for big room simulations, such as theatres and churches, this restriction shows increasing sampling frequencies and the computation times as $\Delta \nu$ decreases. Therefore, when a small spatial discretisation is required due to the room model or the simulation specifications, $\Delta t$ becomes very small, and many iterations are required to evolve the system through time. Additionally, if larger time steps are used to decrease the simulation time, then the error of the numerical solution is increased. To overcome these limitations and to improve the prediction accuracy, some alternative finite-difference schemes have been investigated 


\begin{tabular}{|c|c|c|}
\hline Volume $\left(\mathrm{m}^{3}\right)$ & $\mathrm{D}$ & $\Delta \nu_{\min } /(\Delta t)^{\frac{1}{2}}$ \\
\hline $1 \times 1 \times 1$ & 75.56 & 21.29 \\
$2 \times 2 \times 2$ & 151.11 & 30.11 \\
$3 \times 3 \times 3$ & 226.67 & 36.88 \\
$4 \times 4 \times 4$ & 302.22 & 42.58 \\
$5 \times 5 \times 5$ & 377.78 & 47.61 \\
$6 \times 6 \times 6$ & 453.33 & 52.15 \\
$7 \times 7 \times 7$ & 528.89 & 56.33 \\
$8 \times 8 \times 8$ & 604.44 & 60.22 \\
$9 \times 9 \times 9$ & 680.00 & 63.87 \\
$10 \times 10 \times 10$ & 755.56 & 67.33 \\
\hline
\end{tabular}

Table 5.1. Minimum values of the spatial step size $\Delta \nu_{\text {min }}$ for a given temporal discretisation $\Delta t$ in a given 3-D cubic shaped room.

to avoid any stability restrictions either to the size of the time step $\Delta t$ or the spatial resolution $\Delta \nu$ with a higher error order.

\subsubsection{Analysis of other schemes}

In this section some alternatives FDM schemes are reviewed. There are two main types of FDM schemes: explicit and implicit. Its stability conditions and error order to discuss the suitability of each method for the acoustic diffusion equation model implementation are compared.

The first approach is a simple implicit method called backward-time centred-space, which is accurate to $O[(\Delta t)]+O\left[(\Delta \nu)^{2}\right]$. This scheme is similar to the FTCS scheme, but in this case, the resulting scheme becomes unconditionally stable [Strikwerda, 1989]. To improve the accuracy while maintaining no restrictions on the size of the time step, Crank and Nicolson [Crank and Nicolson, 1974] developed an alternative implicit difference scheme from the simple one, which is second order accurate in both time 
and space $O\left[(\Delta t)^{2}\right]+O\left[(\Delta \nu)^{2}\right]$ and is unconditionally stable [Smith, 1978]. Instead of an explicit form, the two previous schemes use an implicit form because at each time level, a set of equations are to be solved simultaneously for determining the nodal function value. The major drawback with these schemes is solving the equations. In the case of 1-D transient problems the tridiagonal matrix algorithm is an easy and fast mathematical solution; however, for the 2-D and 3-D problems, each point to be updated involves four or more neighbouring point values, and these implicit schemes have not an easy and fast solution to implement [Croft and Lilley, 1977]. Alternatively, there are explicit scheme methods that, in trying to obtain an unconditionally stable solution, become either unconditionally unstable or inconsistent with the differential equation that they are intended to approximate. For example, Richardson [Richardson, 1910] proposed a three-time-level scheme for a FDM approximation of the diffusion equation, but unfortunately, this method is unconditionally unstable.

However, Dufort and Frankel (DF) [Dufort and Frankel, 1953] proposed an expansion of Richardson's scheme replacing a term in order to stabilize it.

$$
\begin{aligned}
\frac{w_{i, j, k}^{n+1}-w_{i, j, k}^{n}}{2 \Delta t}= & D\left(\frac{w_{i+1, j, k}^{n}-w_{i, j, k}^{n+1}-w_{i, j, k}^{n-1}+w_{i-1, j, k}^{n}}{(\Delta x)^{2}}\right. \\
& +\frac{w_{i, j+1, k}^{n}-w_{i, j, k}^{n+1}-w_{i, j, k}^{n-1}+w_{i, j-1, k}^{n}}{(\Delta y)^{2}} \\
+ & \left.\frac{w_{i, j, k+1}^{n}-w_{i, j, k}^{n+1}-w_{i, j, k}^{n-1}+w_{i, j, k-1}^{n}}{(\Delta z)^{2}}\right)-c m w_{i}^{n} .
\end{aligned}
$$

The resulting scheme has a truncation error of order $O\left[(\Delta t)^{2}\right]+O\left[(\Delta \nu)^{2}\right]+$ $O\left[(\Delta t)^{2}(\Delta \nu)^{-2}\right]$ and is unconditionally stable although it is explicit. This explicit and consistent scheme for parabolic equations is convergent only if $(\Delta t)^{2}(\Delta \nu)^{-2}$ tends to zero as $\Delta t$ and $\Delta \nu$ approach zero [Necati, 1994]. These attractive features in the DF scheme allow this method to be the most suitable to apply to the acoustic diffusion equation model. 


\subsubsection{Dufort-Frankel scheme}

\subsubsection{Governing equation discretisation}

In this section, the 3-D problem governed by the acoustic diffusion equation will be developed using DF scheme. For simplification, let us define $\beta_{0_{\nu}}=$ $(2 D \Delta t) /(\Delta \nu)^{2}$, and $\beta_{0}=\sum_{\nu=[x, y, z]} \beta_{0_{\nu}}$, so the finite-difference scheme is as follows,

$$
\begin{aligned}
w_{i, j, k}^{n+1}\left(1+\beta_{0}\right) & =w_{i, j, k}^{n-1}\left(1-\beta_{0}\right)-2 \Delta t c m w_{i, j, k}^{n} \\
& +\beta_{0 x}\left(w_{i+1, j, k}^{n}+w_{i-1, j, k}^{n}\right) \\
& +\beta_{0_{y}}\left(w_{i, j+1, k}^{n}+w_{i, j-1, k}^{n}\right) \\
& +\beta_{0_{z}}\left(w_{i, j, k+1}^{n}+w_{i, j, k-1}^{n}\right) .
\end{aligned}
$$

The source term, included as a soft source, is added at the suitable position as $w_{i_{s}, j_{s}, k_{s}}^{n+1}=w_{i_{s}, j_{s}, k_{s}}^{\prime n+1}+2 \Delta t P_{i_{s}, j_{s}, k_{s}}^{n}$ where $w_{i_{s}, j_{s}, k_{s}}^{n+1}$ is the value after the discretisation cells were updated using Eq. (5.25). Despite the inherent simplicity of using a hard source, this does not correspond to any analytical solution for the partial differential equations [Strikwerda, 1989].

This scheme is theoretically unconditionally stable [Dufort and Frankel, 1953] however, to investigate the stability of the algorithm when the air absorption is included, Sec. 5.2.3.3 presents the derivation and the corresponding explanations.

\subsubsection{Boundary conditions discretisation}

As in the FTCS scheme, first-order accuracy boundary conditions are directly calculated if forward FDM schemes for Eq. (5.6) and backward FDM schemes for Eq. (5.7) are applied, resulting in Eq. (5.10) and Eq. (5.11). Therefore, to ensure the accuracy of the approximation, a second-order accurate difference of the boundary conditions is desirable. In this case, the central FDM approximations involving first order space derivatives cannot be applied because it causes the complete solution to be unstable [Taylor, 1970]. The FDM approximation of these boundary conditions at the nodes 
$i=0$ and $i=L_{x}$ using the second-order accurate three-point formula of the first derivative at the boundary nodes gives

$$
\begin{aligned}
\frac{D}{2 \Delta x}\left(3 w_{0, j, k}^{n+1}-4 w_{1, j, k}^{n+1}\right. & \left.+w_{2, j, k}^{n+1}\right)+A_{x_{0, j, k}} w_{0, j, k}^{n+1}=0 \\
\frac{D}{2 \Delta x}\left(w_{L_{x-2}, j, k}^{n+1}\right. & \left.-4 w_{L_{x-1}, j, k}^{n+1}+3 w_{L_{x}, j, k}^{n+1}\right) \\
& +A_{x_{L_{x}, j, k}} w_{L_{x}, j, k}^{n+1}=0 .
\end{aligned}
$$

Then the following two expressions are derived, respectively,

$$
\begin{aligned}
w_{0, j, k}^{n+1} & =\frac{4 w_{1, j, k}^{n+1}-w_{2, j, k}^{n+1}}{3+\frac{2 A_{x_{0, j, k}} \Delta x}{D}}, \\
w_{L_{x}, j, k}^{n+1} & =\frac{4 w_{L_{x-1}, j, k}^{n+1}+w_{L_{x-2}, j, k}^{n+1}}{3+\frac{2 A_{x_{L_{x}, j, k}} \Delta}{D}} .
\end{aligned}
$$

The derivation for dimension $y$ and dimension $z$ is straightforward.

Thus, Eq. (5.28) and Eq. (5.29), together with Eq. (5.25) for $i=$ $1,2, \ldots, M_{x-1}$ are the complete FDM approximations with the DF method of the acoustic diffusion equation in 3 -D subjected to mixed boundary conditions.

\subsubsection{Von Neumman analysis}

In this section a Von Neumann analysis to find the stability condition of Eq. (5.25) is performed. This is done by assuming a numerical plane wave of the form $w(\mathbf{x}, t)=\xi^{t / \Delta t} e^{-\iota \mathbf{k}_{0}^{T} \mathbf{x}}$.

After applying the Von Neumann procedure (see Sec. 5.2.1.3) to Eq. (5.25) and some basic algebraical simplifications, the following expression is obtained,

$$
\begin{aligned}
\left(1+\beta_{0}\right) \xi & =\left(1-\beta_{0}\right) \xi^{-1}-2 \Delta t c m \\
& +\sum_{\nu=[x, y, z]} \beta_{0_{\nu}}\left(e^{-\iota k_{0_{\nu}} \Delta \nu}+e^{\iota k_{0_{\nu}} \Delta \nu}\right) .
\end{aligned}
$$


Therefore, Eq. (5.30) is equivalent to

$$
\begin{aligned}
\left(1+\beta_{0}\right) \xi^{2} & -\left[\sum_{\nu=[x, y, z]} 2 \beta_{0_{\nu}} \cos \left(k_{0_{\nu}} \Delta \nu\right)-2 \Delta t c m\right] \xi \\
& +\left(\beta_{0}-1\right)=0 .
\end{aligned}
$$

The roots of this second order polynomial are expressed in the following way,

$$
\begin{aligned}
& \xi=\frac{\sum_{\nu=[x, y, z]} \beta_{0_{\nu}} \cos \left(k_{0_{\nu}} \Delta \nu\right)-\Delta t c m}{1+\beta_{0}} \\
& \pm \frac{\sqrt{\left(1-\beta_{0}^{2}\right)+\left[\sum_{\nu=[x, y, z]} \beta_{0_{\nu}} \cos \left(k_{0_{\nu}} \Delta \nu\right)-\Delta t c m\right]^{2}}}{1+\beta_{0}} .
\end{aligned}
$$

Now, examining these roots in detail, two different cases can be found. In the first case, if $\left(1-\beta_{0}^{2}\right)+\left[\sum_{\nu=[x, y, z]} \beta_{0_{\nu}} \cos \left(k_{0_{\nu}} \Delta \nu\right)-\Delta t c m\right]^{2}$ has nonnegative values, it yields,

$$
\begin{aligned}
\|\xi\| & \leq \frac{\beta_{0}-\Delta t c m+\sqrt{\left(1-\beta_{0}^{2}\right)+\left[\beta_{0}-\Delta t c m\right]^{2}}}{1+\beta_{0}} \\
& =\frac{\beta_{0}-\Delta t c m+\sqrt{1+(\Delta t c m)^{2}-2 \beta_{0} \Delta t c m}}{1+\beta_{0}} .
\end{aligned}
$$

Therefore, in order to ensure stability the following condition has to be met,

$$
\frac{\beta_{0}-\Delta t c m+\sqrt{1+(\Delta t c m)^{2}-2 \beta_{0} \Delta t c m}}{1+\beta_{0}} \leq 1,
$$

which is equivalent to $\beta_{0} \geq-1$. Since $\Delta t$ and $\Delta \nu$ are always strictly positive, that condition always holds. 
Implementation of a DEM by a FDM

In the second case, if $\left(1-\beta_{0}^{2}\right)+\left[\sum_{\nu=[x, y, z]} \beta_{0_{\nu}} \cos \left(k_{0_{\nu}} \Delta \nu\right)-\Delta t c m\right]^{2}$ is negative, it is found that,

$$
\begin{aligned}
\|\xi\|^{2} & =\frac{\left[\sum_{\nu=[x, y, z]} \beta_{0_{\nu}} \cos \left(k_{0_{\nu}} \Delta \nu\right)-\Delta t c m\right]^{2}-1}{\left(1+\beta_{0}\right)^{2}} \\
& +\frac{\beta_{0}^{2}-\left[\sum_{\nu=[x, y, z]} \beta_{0_{\nu}} \cos \left(k_{0_{\nu}} \Delta \nu\right)-\Delta t c m\right]^{2}}{\left(1+\beta_{0}\right)^{2}} \\
\leq & \frac{\beta_{0}^{2}-1}{\left(1+\beta_{0}\right)^{2}},
\end{aligned}
$$

then, the stability condition is achieved if

$$
\frac{\beta_{0}^{2}-1}{1+\beta_{0}^{2}+2 \beta_{0}} \leq 1
$$

condition which is unconditionally true.

Therefore, the DF algorithm to be used in the diffusion equation model that includes the air absorption is unconditionally stable.

\subsection{Evaluation of the implementations}

Both the DF and FTCS algorithms are evaluated in a cubic room using four different absorption distributions on the walls. This geometry is chosen to investigate the convergence and the performance of the implementation. Moreover, the absorption distribution is varied to evaluate the behaviour and the consistency of the predicted results. The results are also compared with the acoustical diffuse ray-tracing and the radiosity predictions. Finally, the computational requirements are discussed. Air absorption was neglected throughout the experiment because it has no significant effect on 
the stability of the implementation when it is incorporated as an additional term in Eq. (4.23).

The room has an $8 \times 8 \times 8 \mathrm{~m}^{3}$ volume and an average absorption coefficient of $1 / 6$. The four different absorption distributions being used are indicated in Table 5.2.

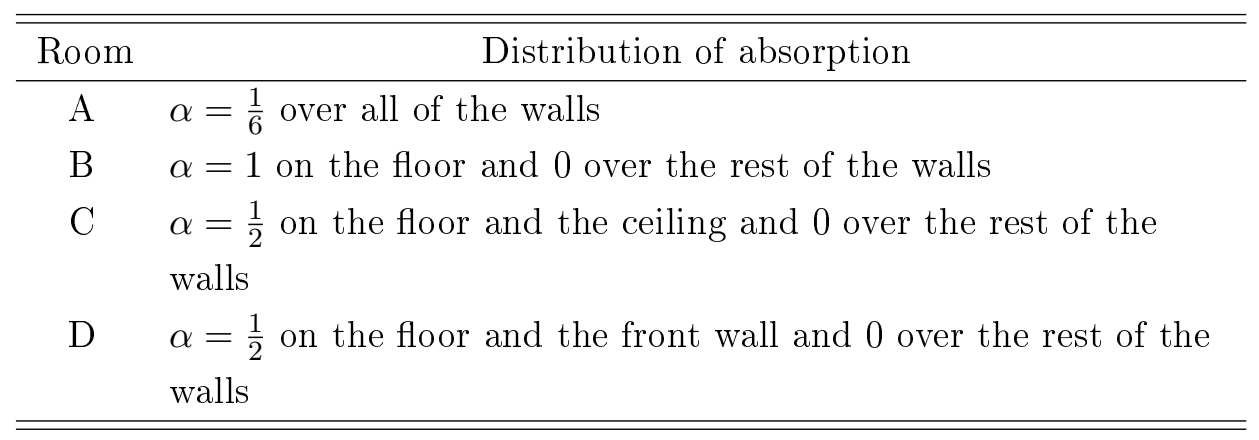

Table 5.2. Distribution of absorption in the cubic rooms.

Several runs were conducted for each distribution with various spatial resolutions and temporal resolutions. A source was located at the centre of the cube with a sound power of $5 \mathrm{~mW}$, and the receiver point was situated inside the enclosure that was $2 \mathrm{~m}$ from each the floor, the front wall and a sidewall. Simulations were run on a PC with an Intel(R) Core(TM)2 Quad CPU 2,5 GHz processor and 2 GB RAM using Matlab ${ }^{\circledR}$.

The energy decay function obtained by the algorithm implementation was used to calculate five room-acoustic parameters: the reverberation time (RT) (obtained from $\left.\mathrm{T}_{30}\right)$, the early decay time (EDT), the definition $\left(\mathrm{D}_{50}\right)$, the clarity $\left(\mathrm{C}_{80}\right)$ and the centre time (TS), which will be useful to perform the analysis. Due to the directional broadening assumption, an intrinsic feature of the diffusion equation model presents an exclusion by the model of both the direct sound and the discrete early reflections, making a diffusion equation model not reliable for calculating energetic balance factors such as definition or clarity. Because the technical literature has shown that the diffusion equation model results in linear energy decays in propor- 
tionate rooms [Picaut et al., 1997], these predictions could be assumed to agree with Barron's revisited theory [Barron, 2000], which is based on the idealised integrated impulse curves according to the Eyring reverberation time equation. Therefore, this information might be used as the expectation values and as a reference to check the plausibility of the diffusion equation implementation results [Vorländer, 2008]. It should be pointed out that this type of estimation obtained using Barron's theory, gives no exact results but only the order of magnitude of these room-acoustic parameters.

\subsubsection{Spatial resolution}

The discretisation of the enclosure is performed in terms of FDM, which means that the entire room is divided into cubic cells whose side is equal to the spatial resolution $\Delta \nu$ in each Cartesian direction. For the specific problem of the diffusion equation, the temporal broadening assumption determines that the size of the cells should be on the order of (or smaller than) one mean free path. Thus, the diffusion model can be applied to very large enclosures with a limited meshing. Regarding the architectural details of the room, unless the details need to be simulated, e.g., a small door or an aperture between two coupled rooms, the cells size should be selected as the maximum possible value in order to increase the computational performance.

The accuracy of the numerical solution is related to both time and space resolutions, as indicated by the error order of each scheme. If the temporal resolution is fixed, this discretisation error decreases as the meshing resolution is increased because the numerical solution approaches the analytical solution. Otherwise, it is desirable to minimise the number of cells used in the discretisation to reduce the computational requirements. The DF scheme is of second-order error in time and space, so it is expected to converge faster than FTCS. As it has been discussed in Sec. 5.2.3.1, the FTCS scheme is of first-order error in time; therefore, a smaller time step $(\Delta t)$ compared to DF is necessary to obtain a similar error. Moreover, a 
larger restriction of FTCS performance lies in its stability condition (see Eq. (5.22)): for a smaller cell, a higher temporal resolution is needed, which subsequently increases the number of iterations needed [Strikwerda, 1989, Necati, 1994]. These issues seem to support the DF scheme as the most suitable choice to simulate the diffusion equation model; however, its most remarkable advantage is its theoretical unconditional stability, which allows one to choose the temporal and the spatial resolutions without restrictions.

To investigate these issues, the DF implementation of the acoustic diffusion equation model was run ten times for each cubic room with a decreasing cell size $\Delta \nu$, from 1.2 to $0.06 \mathrm{~m}$. The temporal resolution was fixed to $(\Delta t)^{-1}=64 \mathrm{~ms}^{-1}$ to ensure that the absolute error is independent of it. The simulations were carried out over $1.0 \mathrm{~s}$, and the estimated values were compared with the calculated data from Barron's revisited theory [Vorländer, 2008] with the influence of the direct field neglected. The obtained values using Barron's formulae are shown in Table 5.3.

\begin{tabular}{ccccc}
\hline \hline $\mathrm{C}_{80}$ & $\mathrm{D}_{50}$ & EDT & $\mathrm{T}_{30}$ & $\mathrm{TS}$ \\
$(\mathrm{dB})$ & $(\%)$ & $(\mathrm{s})$ & $(\mathrm{s})$ & $(\mathrm{ms})$ \\
\hline 1.81 & 43.86 & 1.20 & 1.20 & 86.63 \\
\hline \hline
\end{tabular}

Table 5.3. Parameters calculated with Barron's revisited theory formulae for the cubic room with an average absorption coefficient of $1 / 6$.

In tables 5.4 and 5.5, the predicted values of the room-acoustic parameters of room $A$ are presented for various spatial resolutions. It should be noted that the predicted values of each room-acoustic parameter converges to a finite value as the spatial resolution increases.

The trends are highly similar for the other room scenarios. Each parameter considered converges to a finite value as the number of cells increases. To support this evidence, the error regarding Barron's reference model is also evaluated in terms of the just noticeable differences (jnds) concept (see 


\begin{tabular}{ccccc}
\hline \hline $\begin{array}{c}\Delta \nu \\
(\mathrm{m})\end{array}$ & $\begin{array}{c}\text { Number } \\
\text { of cells }\end{array}$ & $\begin{array}{c}\mathrm{C}_{80} \\
(\mathrm{~dB}(\mathrm{~dB}))\end{array}$ & $\begin{array}{c}\mathrm{D}_{50} \\
(\%(\%))\end{array}$ & $\begin{array}{c}\text { EDT } \\
(\mathrm{s}(\%))\end{array}$ \\
\hline 1.2 & 296 & $4.21(2.40)$ & $56.66(12.80)$ & $0.90(24.46)$ \\
1 & 512 & $2.95(1.14)$ & $50.35(6.49)$ & $1.06(11.48)$ \\
0.8 & 1,000 & $2.76(0.95)$ & $49.47(5.61)$ & $1.09(9.03)$ \\
0.6 & 2,370 & $2.90(1.08)$ & $50.48(6.62)$ & $1.08(9.27)$ \\
0.5 & 4,096 & $2.30(0.48)$ & $46.68(2.84)$ & $1.14(4.92)$ \\
0.4 & 8,000 & $2.17(0.35)$ & $45.92(2.07)$ & $1.15(3.56)$ \\
0.2 & 64,000 & $1.92(0.11)$ & $44.52(0.67)$ & $1.19(0.83)$ \\
0.1 & 512,000 & $1.80(0.01)$ & $43.88(0.02)$ & $1.20(0.50)$ \\
0.08 & $1,000,000$ & $1.78(0.03)$ & $43.75(0.10)$ & $1.20(0.75)$ \\
0.06 & $2,370,371$ & $1.79(0.03)$ & $43.77(0.08)$ & $1.20(0.65)$ \\
\hline \hline
\end{tabular}

Table 5.4. Parameters predictions $\left(C_{80}, D_{50}\right.$ and EDT) with $D F$ scheme and their corresponding percentage error with reference model (indicated inside the brackets) with varying cell size for the cubic room $A$ and $(\Delta t)^{-1}=64 \mathrm{~ms}^{-1}$.

Sec. 2.3.3.6). According to ISO/DIS 3382 [ISO, 2009], the subjective threshold for the reverberation time, the early decay time and the definition index is $5 \%$, whereas for the clarity index it is required to be $1 \mathrm{~dB}$, and for the centre time is $10 \mathrm{~ms}$ (see Table 2.2). All of the parameters have values less than the subjective threshold for a cell size below $0.5 \mathrm{~m}$ as shown in Tables 5.4 and 5.5.

\subsubsection{Temporal resolution}

Although the DF algorithm has been shown to always satisfy the stability criterion because it is unconditionally stable, further predictions were made to investigate the effect of the time resolution on the convergence and the accuracy of the numerical solution. For these simulations, seven temporal resolutions with increasing values from $(\Delta t)^{-1}=1 \mathrm{~ms}^{-1}$ to $64 \mathrm{~ms}^{-1}$ for each 


\begin{tabular}{ccccc}
\hline \hline $\begin{array}{c}\Delta \nu \\
(\mathrm{m})\end{array}$ & $\begin{array}{c}\text { Number } \\
\text { of cells }\end{array}$ & $\begin{array}{c}\text { RT } \\
(\mathrm{s}(\%))\end{array}$ & $\begin{array}{c}\text { TS } \\
(\mathrm{ms}(\mathrm{ms}))\end{array}$ & $\begin{array}{c}(\Delta t)^{2}(\Delta \nu)^{-2} \\
10^{-8}\end{array}$ \\
\hline 1.2 & 296 & $0.91(23.90)$ & $61.36(25.27)$ & 0.01 \\
1 & 512 & $1.06(10.97)$ & $73.56(13.07)$ & 0.02 \\
0.8 & 1,000 & $1.09(8.42)$ & $75.68(10.94)$ & 0.03 \\
0.6 & 2,370 & $1.09(8.48)$ & $74.27(12.36)$ & 0.06 \\
0.5 & 4,096 & $1.14(4.58)$ & $80.90(5.73)$ & 0.09 \\
0.4 & 8,000 & $1.16(3.29)$ & $82.44(4.19)$ & 0.15 \\
0.2 & 64,000 & $1.19(0.71)$ & $85.39(1.24)$ & 0.61 \\
0.1 & 512,000 & $1.20(0.56)$ & $86.80(0.17)$ & 2.44 \\
0.08 & $1,000,000$ & $1.20(0.79)$ & $87.04(0.41)$ & 3.81 \\
0.06 & $2,370,371$ & $1.20(0.73)$ & $87.05(0.43)$ & 6.78 \\
\hline \hline
\end{tabular}

Table 5.5. Parameters predictions (RT, TS) and truncation error with DF scheme and their corresponding percentage error with reference model (indicated inside the brackets) with varying cell size for the cubic room $A$ and $(\Delta t)^{-1}=64 \mathrm{~ms}^{-1}$.

$\Delta \nu$ considered in the previous experiment (see Table 5.5) were used.

As occurred in the case with various $\Delta \nu$, the room-acoustic parameter predictions always converge to a fixed value when the time resolution increases. To study the evolution of the results, the percentage differences between the predictions in consecutive temporal resolution situations are evaluated, i.e. the difference between two values divided by the average of the two values is shown as a percentage. In Tables 5.6 and 5.7, it is observed that these differences decrease when the temporal resolution is increased, using $\Delta \nu=0.4 \mathrm{~m}$ and $\Delta \nu=0.2 \mathrm{~m}$ to mesh the cubic room $A$ respectively. Among the different parameters, $\mathrm{C}_{80}$ is the slowest to converge; however, the percentage difference between fourth and fifth predictions $\left((\Delta t)^{-1}=8\right.$ $\mathrm{ms}^{-1}$ and $16 \mathrm{~ms}^{-1}$ ) using $\Delta \nu=0.4 \mathrm{~m}$ is lower than $1 \%$ for all room-acoustic parameters. The values presented for a spatial step of $0.2 \mathrm{~m}$ also follow the same convergence trend, resulting in a percentage difference for consecutive 
predictions below $1 \%$ between fifth and sixth predictions.

\begin{tabular}{cccccc}
\hline \hline $\begin{array}{c}n^{t h}-(n+1)^{s t} \\
\text { prediction }\end{array}$ & $\begin{array}{c}\mathrm{C}_{80} \\
(\%)\end{array}$ & $\begin{array}{c}\mathrm{D}_{50} \\
(\%)\end{array}$ & $\begin{array}{c}\text { EDT } \\
(\%)\end{array}$ & $\begin{array}{c}\mathrm{RT} \\
(\%)\end{array}$ & $\begin{array}{c}\mathrm{TS} \\
(\%)\end{array}$ \\
\hline $1-2$ & 20.26 & 6.38 & 12.06 & 14.17 & 11.05 \\
$2-3$ & 12.48 & 4.06 & 2.61 & 2.75 & 5.10 \\
$3-4$ & 6.88 & 2.41 & 0.62 & 0.65 & 2.46 \\
$4-5$ & 0.22 & 0.09 & 0.18 & 0.16 & 0.01 \\
$5-6$ & 0.05 & 0.03 & 0.04 & 0.04 & 0.06 \\
$6-7$ & 0.00 & 0.01 & 0.01 & 0.01 & 0.02 \\
\hline \hline
\end{tabular}

Table 5.6. Percentage differences between the parameters predictions in consecutive temporal resolution situations using $D F$ scheme with varying $(\Delta t)^{-1}$ for the cubic room $A$ and cell size $\Delta \nu=0.4 \mathrm{~m}$.

Moreover, the estimated values of the parameters versus $(\Delta t)^{-1}$ are shown in Tables 5.8- 5.11. Again, data inside the brackets are the difference between this model and the Barron's reference model. Although the presented tables are of prediction values of room $\mathrm{A}$, the trends are similar for all room scenarios.

Moreover these predicted values are below the subjective difference limens specified in ISO/DIS 3382 compared to the results from the Barron's reference model (from $(\Delta t)^{-1}=4 \mathrm{~ms}^{-1}$ with $\Delta \nu=0.4 \mathrm{~m}$ or $0.2 \mathrm{~m}$ ). The error decreases as the temporal resolution is increased in all simulated configurations. It is of note that for each spatial resolution, there is a time resolution value from which the error does not decrease even if the temporal resolution is increased.

This presented observational evidence combined with the author's experience in many other simulations might suggest an empirical criterion to choose the spatial and time resolution: an order of magnitude of $10^{-8}$ for the relation $(\Delta t)^{2}(\Delta \nu)^{-2}$ ensures that the predictions converge to a fixed 


\begin{tabular}{cccccc}
\hline \hline $\begin{array}{c}n^{t h}-(n+1)^{s t} \\
\text { prediction }\end{array}$ & $\begin{array}{c}\mathrm{C}_{80} \\
(\%)\end{array}$ & $\begin{array}{c}\mathrm{D}_{50} \\
(\%)\end{array}$ & $\begin{array}{c}\mathrm{EDT} \\
(\%)\end{array}$ & $\begin{array}{c}\mathrm{RT} \\
(\%)\end{array}$ & $\begin{array}{c}\mathrm{TS} \\
(\%)\end{array}$ \\
\hline $1-2$ & 14.48 & 7.22 & 12.05 & 10.46 & 24.23 \\
$2-3$ & 21.95 & 2.48 & 11.81 & 13.68 & 10.25 \\
$3-4$ & 13.6 & 4.03 & 2.58 & 2.67 & 4.76 \\
$4-5$ & 7.64 & 2.42 & 0.62 & 0.63 & 2.30 \\
$5-6$ & 0.14 & 0.00 & 0.17 & 0.16 & 0.09 \\
$6-7$ & 0.11 & 0.03 & 0.04 & 0.04 & 0.05 \\
\hline \hline
\end{tabular}

Table 5.7. Percentage differences between the parameters predictions in consecutive temporal resolution situations using DF scheme with varying $(\Delta t)^{-1}$ for the cubic room $A$ and cell size $\Delta \nu=0.2 \mathrm{~m}$

value with a very low error. Accordingly, to configure a simulation, a spatial resolution is fixed, and a temporal resolution is calculated with this empirical criterion to obtain low error results with the minimum computational requirements.

\subsubsection{Computational efficiency}

The memory requirement depends exclusively on the spatial resolution needed for the particular simulation. The FTCS scheme (see Eq. (5.5)) needs to store only the neighbour node values at the previous time level to calculate the unknown value at the present time. Therefore, the memory consumption, solved by the mathematical derivation, is associated with a memory complexity of order $2 n$, where $n$ is the total number of cells in which the model is discretised. Meanwhile, the DF scheme (see Eq. (5.25)) needs to store the value of the sound energy density in the two time steps before the one that is being calculated. In theory, this requirement results in a memory complexity of order $3 n$. However, it is possible to implement an algorithm to reduce the requirements to $2 n$, similar to FTCS, by using the same matrix $w^{n-1}$ to store the calculated $w^{n+1}$ values in each iteration [Strikwerda, 


\begin{tabular}{cccc}
\hline \hline $\begin{array}{c}(\Delta t)^{-1} \\
\left(\mathrm{~ms}^{-1}\right)\end{array}$ & $\begin{array}{c}\mathrm{C}_{80} \\
(\mathrm{~dB}(\mathrm{~dB}))\end{array}$ & $\begin{array}{c}\mathrm{D}_{50} \\
(\%(\%))\end{array}$ & $\begin{array}{c}\mathrm{EDT} \\
(\mathrm{s}(\%))\end{array}$ \\
\hline 1 & $3.22(1.40)$ & $52.19(8.33)$ & $0.99(17.45)$ \\
2 & $2.62(0.81)$ & $48.96(5.11)$ & $1.11(6.85)$ \\
4 & $2.32(0.50)$ & $47.02(3.16)$ & $1.14(4.39)$ \\
8 & $2.16(0.35)$ & $45.90(2.04)$ & $1.15(3.79)$ \\
16 & $2.17(0.35)$ & $45.94(2.09)$ & $1.15(3.62)$ \\
32 & $2.17(0.35)$ & $45.93(2.07)$ & $1.15(3.57)$ \\
64 & $2.17(0.35)$ & $45.92(2.07)$ & $1.15(3.56)$ \\
\hline \hline
\end{tabular}

Table 5.8. Parameters predictions $\left(C_{80}, D_{50}\right.$ and EDT) using DF scheme and their corresponding percentage error with reference model (indicated inside the brackets) with varying $(\Delta t)^{-1}$ for the cubic room $A$ and cell size $\Delta \nu=0.4 \mathrm{~m}$.

1989]. Moreover, it is always possible to select a higher $\Delta \nu$ for a fixed $\Delta t$ using the DF scheme than the FTCS, which consumes less memory.

The elapsed time of the diffusion equation algorithm based on a FDM scheme depends on the spatial and the temporal resolutions as well as the simulated length of the impulse response. As expected from above, in all of the simulations performed using both the FTCS and the DF implementations, a similar elapsed time was observed for both methods when the configuration parameters were the same. Fig. 5.2 shows the DF implementation elapsed time versus the number of cells for different temporal resolutions with a response length of $1.0 \mathrm{~s}$ in room $A$. The run times for predictions for the other room scenarios are also similar.

However, the stability condition of FTCS (see Eq. (5.22)) is its main limitation, which increases the memory consumption and the elapsed time compared to DF. The computational efficiency comparison between FTCS and DF cannot be directly made using the same spatial and temporal resolution. If a spatial resolution is fixed, the DF scheme can always use 


\begin{tabular}{cccc}
\hline \hline $\begin{array}{c}(\Delta t)^{-1} \\
\left(\mathrm{~ms}^{-1}\right)\end{array}$ & $\begin{array}{c}\mathrm{RT} \\
(\mathrm{s}(\%))\end{array}$ & $\begin{array}{c}\mathrm{TS} \\
(\mathrm{ms}(\mathrm{ms}))\end{array}$ & $\begin{array}{c}(\Delta t)^{2}(\Delta \nu)^{-2} \\
10^{-8}\end{array}$ \\
\hline 1 & $0.97(19.07)$ & $68.37(18.26)$ & 625 \\
2 & $1.11(6.72)$ & $76.36(10.26)$ & 156.25 \\
4 & $1.15(4.12)$ & $80.36(6.26)$ & 39.06 \\
8 & $1.15(3.50)$ & $82.36(4.27)$ & 9.76 \\
16 & $1.16(3.34)$ & $82.37(4.26)$ & 2.44 \\
32 & $1.16(3.30)$ & $82.42(4.21)$ & 0.61 \\
64 & $1.16(3.29)$ & $82.44(4.19)$ & 0.15 \\
\hline \hline
\end{tabular}

Table 5.9. Parameters predictions (RT, TS) and truncation error using DF scheme and their corresponding percentage error with reference model (indicated inside the brackets) with varying $(\Delta t)^{-1}$ for the cubic room $A$ and cell size $\Delta \nu=0.4$ $m$.

a temporal resolution lower than the FTCS scheme, resulting in a lower elapsed time. Moreover, as presented in Sec. 5.2.3, the error order of the DF scheme is higher than that of FTCS scheme, resulting in a lower error for the DF scheme predictions with the same temporal and spatial resolutions.

\subsubsection{Comparison with other methods}

In this section, the diffusion equation model results using both DF and FTCS are compared to the other well-known acoustic geometrical-based simulation methods, which also use diffuse reflections on the boundaries, such as the radiosity method and the diffuse ray-tracing method. Table 5.12 presents the results obtained from the aforementioned geometrical-based methods, which were obtained from Nosal et al. [Nosal et al., 2004a]. Table 5.12 also includes the predicted room-acoustic parameters using both the FTCS and the DF schemes with $\Delta \nu=0.2 \mathrm{~m}$ and their corresponding temporal resolution. 


\begin{tabular}{cccc}
\hline \hline $\begin{array}{c}(\Delta t)^{-1} \\
\left(\mathrm{~ms}^{-1}\right)\end{array}$ & $\begin{array}{c}\mathrm{C}_{80} \\
(\mathrm{~dB}(\mathrm{~dB}))\end{array}$ & $\begin{array}{c}\mathrm{D}_{50} \\
(\%(\%))\end{array}$ & $\begin{array}{c}\mathrm{EDT} \\
(\mathrm{s}(\%))\end{array}$ \\
\hline 1 & $1.93(1.62)$ & $40.45(8.49)$ & $0.90(24.52)$ \\
2 & $2.97(1.15)$ & $48.70(4.85)$ & $1.02(14.84)$ \\
4 & $2.38(0.57)$ & $47.51(3.65)$ & $1.15(4.15)$ \\
8 & $2.08(0.26)$ & $45.63(1.77)$ & $1.18(1.65)$ \\
16 & $1.92(0.11)$ & $44.54(0.69)$ & $1.18(1.04)$ \\
32 & $1.92(0.11)$ & $44.54(0.67)$ & $1.18(0.87)$ \\
64 & $1.92(0.11)$ & $44.52(0.67)$ & $1.19(0.83)$ \\
\hline \hline
\end{tabular}

Table 5.10. Parameters predictions $\left(C_{80}, D_{50}\right.$ and EDT) using DF scheme and their corresponding percentage error with reference model (indicated inside the brackets) with varying $(\Delta t)^{-1}$ for the cubic room $A$ and cell size $\Delta \nu=0.2 \mathrm{~m}$.

In all methods, the longest $\mathrm{RT}$ is in room $A$, where the absorption is uniform. The shortest RT is in room $B$, which has a high absorption factor at the floor near the receiver. The different $\mathrm{RT}$ values in each room indicates that the effect of different absorption distributions is well simulated by the implementations of the acoustic diffusion equation model. For higher RT, lower $\mathrm{C}_{80}$ and $\mathrm{D}_{50}$ values are obtained as expected, because a slower energy decay curve means more late energy, resulting in the lower early-to-late energy ratios. The TS predicted values are also consistent with the RT results.

The predicted values using FTCS and DF diffusion equation implementations are compared to the results from radiosity and diffuse ray-tracing in terms of jnds. Although the FTCS predictions for rooms $A$ and $D$ are below the subjective difference limens as compared to both geometrical-based methods, the differences are observable over the limens in all room-acoustic parameters for rooms $B$ and $C$ for both geometrical-based methods. It is straightforward to state that the DF predictions for all room scenarios are below the subjective difference limens compared to both geometrical-based 


\begin{tabular}{cccc}
\hline \hline $\begin{array}{c}(\Delta t)^{-1} \\
\left(\mathrm{~ms}^{-1}\right)\end{array}$ & $\begin{array}{c}\mathrm{RT} \\
(\mathrm{s}(\%))\end{array}$ & $\begin{array}{c}\mathrm{TS} \\
(\mathrm{ms}(\mathrm{ms}))\end{array}$ & $\begin{array}{c}(\Delta t)^{2}(\Delta \nu)^{-2} \\
10^{-8}\end{array}$ \\
\hline 1 & $0.47(24.71)$ & $56.21(30.42)$ & 2500 \\
2 & $1.00(16.40)$ & $71.71(14.92)$ & 625 \\
4 & $1.15(4.12)$ & $79.45(7.17)$ & 156.25 \\
8 & $1.18(1.53)$ & $83.33(3.30)$ & 39.06 \\
16 & $1.18(0.90)$ & $85.27(1.36)$ & 9.76 \\
32 & $1.19(0.75)$ & $85.34(1.29)$ & 2.44 \\
64 & $1.19(0.75)$ & $85.39(1.24)$ & 0.61 \\
\hline \hline
\end{tabular}

Table 5.11. Parameters predictions (RT, TS) and truncation error using DF scheme and their corresponding percentage error with reference model (indicated inside the brackets) with varying $(\Delta t)^{-1}$ for the cubic room $A$ and cell size $\Delta \nu=0.2$ $m$.

methods. In particular, acoustical radiosity gives quite similar predictions to the DF predictions. The parameter that exhibits the greatest difference between predictions is TS; however, most values are below $10 \mathrm{~ms} . \mathrm{RT}, \mathrm{D}_{50}$ and EDT are all within $4 \%$, and $\mathrm{C}_{80}$ is below $0.1 \mathrm{~dB}$. Although similar results are obtained when the diffuse ray-tracing results are compared with the DF predicted values, these differences are higher than those obtained using radiosity method. These differences regarding geometrical-based methods lie in the different manners in which the diffuse reflection is incorporated in the models: the radiosity method and the acoustic diffusion equation model scatter rays in all directions, whereas random directions are used in diffuse ray-tracing [Nosal et al., 2004a]. These experimental results support the hypothesis that there are considerable differences between FTCS and DF implementations, which are associated with the different error orders of each scheme. 


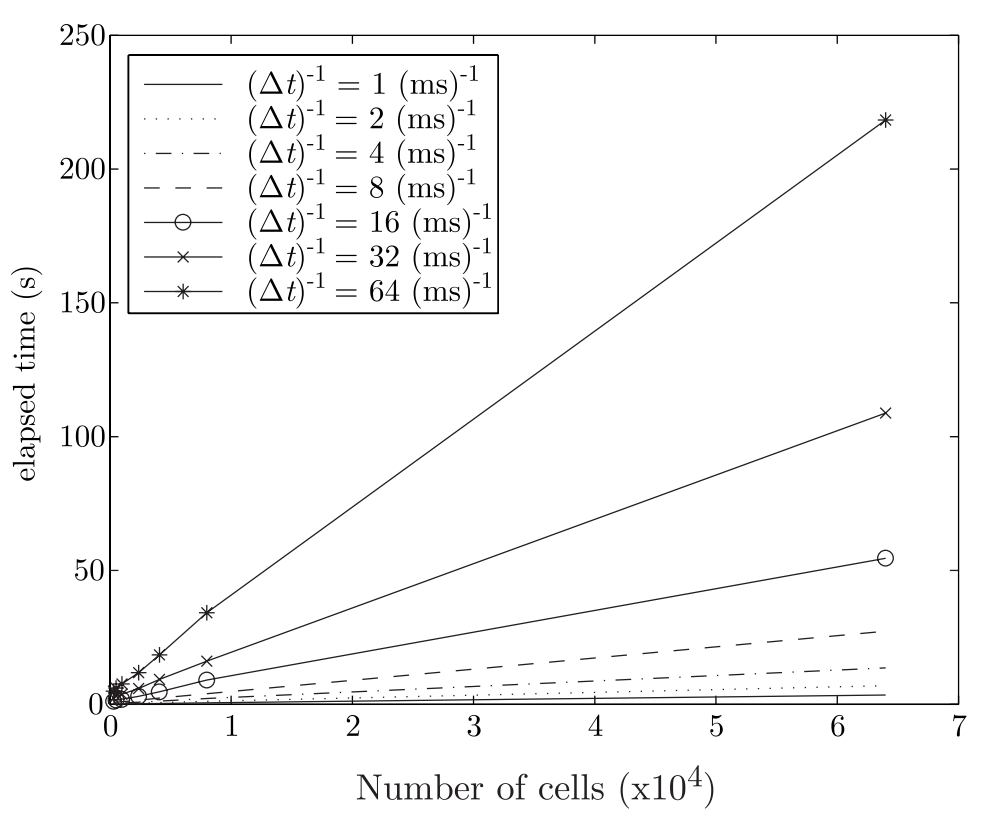

Figure 5.2. Variation of elapsed time with number of cells for cubic room A with the Dufort-Frankel implementation.

\subsection{Summary}

In this chapter, the implementation of the acoustic diffusion equation model for room-acoustic simulations using a finite-difference method (FDM) has been investigated. Both the forward-time centre-space (FTCS) and the Dufort-Frankel (DF) explicit FDM schemes have been used, and their performance in cubic-shaped rooms have been evaluated.

Compared with the other FDM schemes, the DF scheme has been shown to be the most suitable and simple solution, and it is easy to implement with no stability restrictions and a second-order accuracy. The air absorption effect has been added to the implementation, and its stability condition has been derived so that the algorithm remains unconditionally stable and that the convergence of the algorithm is not affected. 


\begin{tabular}{|c|c|c|c|c|c|c|c|c|c|c|c|c|}
\hline \multirow{3}{*}{ (a) } & \multirow{2}{*}{\multicolumn{2}{|c|}{$\begin{array}{r}\mathrm{C}_{80} \\
\operatorname{Room}(\mathrm{dB})\end{array}$}} & \multirow{2}{*}{$\begin{array}{l}D_{50} \\
(\%)\end{array}$} & \multicolumn{2}{|c|}{ EDT RT } & \multirow{2}{*}{$\begin{array}{l}\mathrm{TS} \\
(\mathrm{ms})\end{array}$} & \multirow{2}{*}{\multicolumn{2}{|c|}{$\begin{array}{r}\mathrm{C}_{80} \\
\text { Room }(\mathrm{dB})\end{array}$}} & \multirow{2}{*}{$\begin{array}{l}\mathrm{D}_{50} \\
(\%)\end{array}$} & \multicolumn{2}{|c|}{ EDT RT } & \multirow{2}{*}{$\begin{array}{l}\mathrm{TS} \\
(\mathrm{ms})\end{array}$} \\
\hline & & & & (s) & (s) & & & & & $(\mathrm{s})$ & (s) & \\
\hline & $A$ & 1.73 & 43.61 & 1.22 & 1.22 & $87.87(\mathrm{c})$ & $A$ & 2.00 & 45.87 & 1.23 & 1.20 & 94.67 \\
\hline & $B$ & 5.89 & 63.07 & 0.70 & 0.70 & 50.3 & $B$ & 2.85 & 49.88 & .08 & 1.06 & 84.50 \\
\hline & $C$ & 4.84 & 58.61 & 0.80 & 0.80 & 57.0 & $C$ & 2.39 & 47. & .17 & 1.16 & 89.70 \\
\hline & $D$ & 1.80 & 43.99 & 1.21 & 1.21 & 87.00 & $D$ & 2.35 & 47. & .17 & 1.16 & 90.20 \\
\hline (b) & $A$ & 2.08 & 45.6 & 18 & 1.18 & 85. & $A$ & 2.02 & 45.8 & .22 & 1.23 & 94.50 \\
\hline & $B$ & 2.88 & 49.5 & .05 & 1.05 & 75.96 & $B$ & 2.83 & 49.6 & .13 & 0.98 & 84.95 \\
\hline & $C$ & 2.31 & 46.4 & 1.12 & 1.12 & 82.2 & C & 2.31 & 46. & .18 & 1.16 & 91.38 \\
\hline & $D$ & 2.33 & 47.40 & 1.16 & 1.17 & 82.5 & $D$ & 2.24 & 46. & 1.18 & 1.18 & 91.31 \\
\hline
\end{tabular}

Table 5.12. Parameters predictions for the four cubic rooms using: (a) FTCS with $\Delta \nu=0.2 \mathrm{~m}$ and $(\Delta t)^{-1}=96 \mathrm{~ms}^{-1}$; (b) $D F$ with $\Delta \nu=0.2 \mathrm{~m}$ and $(\Delta t)^{-1}=8 \mathrm{~ms}^{-1}$; (c) acoustical radiosity with 150 patches and $(\Delta t)^{-1}=24 \mathrm{~ms}^{-1}$ and (d) diffuse ray-tracing with average over 64 runs with 1 million rays [diffuse reflection, 500 reflections, $24 \mathrm{kHz},(0.1 \mathrm{~m})^{3}$ receiver].

Using both FTCS and DF implementations, several simulations have been carried out with different absorption distributions in a cubic room, and the room-acoustic parameters have been obtained. Experimental validations give rise to a possible empirical criterion that can be used to calculate an empirical temporal resolution value from a fixed spatial resolution of the DF implementation, which helps one obtaining accurate predictions.

The comparisons to some geometrical-based methods, which also assume diffuse reflections, suggest that the DF implementation of the acoustic diffusion equation model obtains highly accurate predictions in this type of enclosure.

The elapsed time increases as the number of cells $n$ increases and they have a memory complexity of order $2 n$. Although both FTCS and DF implementations have the same memory and time complexities, the unconditionally stable feature of DF allows one to discretise the room with fewer 
cells than FTCS, resulting in a lower memory consumption and a decreased elapsed time.

It can be concluded that the DF FDM scheme for the diffusion equation model is a reliable method for performing accurate simulations with low computational complexity and calculation time.

In the following chapter (Chap. 6), a deeper investigation of the diffusion equation model implemented by FDM to reinforce these affirmations is carried out. The proposed FDM schemes are used to numerically check the theoretical advantages and limitations of the diffusion equation model. Also, a comparison between the diffusion equation model and the room-acoustic rendering equation in a complex scenario using numerical simulations and measurement data is done. 


\section{Evaluation and Applicability of the Diffusion Equation Model for Room Acoustics Simulations}

The sPecific Assumptions, both directional and temporal broadening that define the acoustic diffusion model result in some theoretical advantages and limitations that were discussed in Sec. 4.8.

The purpose of this chapter is to evaluate these features of the acoustic diffusion model for room acoustics simulations using the finite-difference implementations developed in Chap. $5^{1}$.

\subsection{Introduction}

The previous chapter examined how a diffusion equation model could be implemented by a finite-difference scheme, with a number of important factor being considered. In this chapter the numerical implementations of the acoustic diffusion equation model; forward-time centred-space (FTCS)

\footnotetext{
${ }^{1}$ Portions of this chapter constitute the major part of a paper published on the Journal of Acoustical Society of America [Escolano et al., 2010] and several convention papers [Navarro et al., 2010a,b, 2011].
} 
and Dufort-Frankel (DF) are tested for two kinds of rooms: rectangular and complex shapes. Rectangular rooms are simple enclosed spaces with cubic or parallelepiped shape where none of the dimensions is much larger than the other. Complex shape rooms are simple enclosed spaces with irregular shape, i.e. with non proportional relation between dimensions and with some surfaces not parallel between them.

Prior works have suggested that the acoustic diffusion equation model properly models the late part of the room impulse response with low computational cost [Picaut et al., 1997, Valeau et al., 2006]. In particular, the diffusion equation is reasonable accurate while the absorption involved is low. As it has been shown in Chap. 4, the acoustic diffusion equation model is an approximation of the acoustic radiative transfer model. To derive the diffusion equation the directional and temporal broadening assumptions are applied. This results in some theoretical advantages and limitations. This study continues the efforts by prior authors investigating some of these limitations.

This chapter focuses in analysing and discussing the applicability of the acoustic diffusion equation model for room acoustics simulations regarding to the accuracy of the temporal and spatial dependence of the predicted sound field distribution.

It is organized as follows: The first group of evaluations is done in rectangular rooms. Firstly, in Sec. 6.2.1 this study evaluates the correct behaviour of the acoustic diffusion equation model including the additional air absorption term. Secondly, incorporating the air absorption effects, in Sec. 6.2.2 this work compares the diffusion model with other two well-known geometrical acoustics models that also assume total diffuse reflections. Finally, an in depth investigation about the validity of the diffusion model to predict the late reverberance is achieved (see Sec. 6.2.3).

The second group of evaluations is done in a complex shape room (see Sec. 6.3). This study presents some comparisons of predicted values from the acoustic diffusion equation and other geometrical acoustics models as 
well as experimental results obtained from a round robin test in a real room. Finally, the chapter is summarized and discussed.

\subsection{Simulations in rectangular rooms}

In this section, several simulations in rectangular rooms using different configurations are presented. This study evaluates the performance of the acoustic diffusion equation model including the air absorption effects comparing with other geometrical acoustics models.

\subsubsection{Air absorption effects}

\subsubsection{Introduction}

Atmospheric attenuation of sound describes the process of energy dissipation along wave propagation in the form of a heat release. As it has been presented in Sec. 2.2.7.2.1, a still atmosphere attenuates sound as a function of frequency, temperature, relative humidity, and propagation distance. Therefore, it is a physic phenomenon that have to be modelled in room acoustics simulation algorithms to obtain accurate predictions. This attenuation factor is pointed to be mainly important at high frequencies and in large enclosures [Bass et al., 1972].

In this section the correct behaviour of the acoustic diffusion equation model with the additional absorption term is studied comparing predicted results using a rectangular dimensions room and different air absorption coefficients. The surfaces absorption is varied over a wide range of values with simulations for several atmospheric attenuation values.

\subsubsection{Analysis}

Some simulations have been carried out using the FTCS implementation of the model (see Sec. 5.2.1). The numerical solution has been implemented in Matlab @to run time-dependent simulations allowing energy decay curves 
prediction in all mesh points at the same time. A large shoe-box room is used, which dimensions are $10 \mathrm{~m}$ wide, $20 \mathrm{~m}$ long and $10 \mathrm{~m}$ high. The boundaries absorption were homogeneously distributed, changing from 0.1 to 0.9 in each run, adopting modified absorption factor $\left(A_{M}\right)$. To meet the stability conditions Eq. (5.21) and to obtain low error, the configuration parameters are $\Delta t=1.6 \cdot 10^{-5} \mathrm{~s}$ and $\Delta x=\Delta y=\Delta z=0.3 \mathrm{~m}$. The room atmospheric attenuation conditions has been varied between four different values; no absorption and absorption values at $500 \mathrm{~Hz}, 1,000 \mathrm{~Hz}$, and 2,000 $\mathrm{Hz}$. The air absorption coefficients have been calculated with the numerical expressions founded in [Bass et al., 1972] (see Table 2.1).

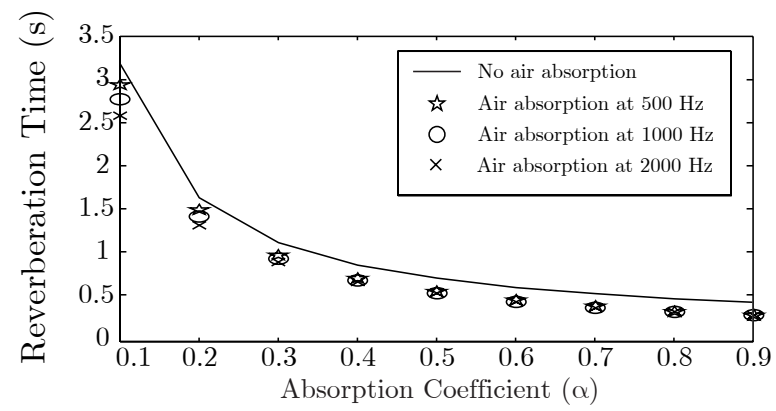

Figure 6.1. Reverberation times values (RT) obtained as function of an homogeneous distribution of absorbing material for a room with size $10 \times 20 \times 10 \mathrm{~m}^{3}$, using no air absorption (solid line), air absorption at $500 \mathrm{~Hz}(\star)$, air absorption at 1,000 $\mathrm{Hz}(\bigcirc)$ and air absorption at 2,000 $\mathrm{Hz}(\times)$ with Modified boundary condition.

The reverberation time values (RT) obtained from the predictions in each cell of the discretised room, were averaged. Fig. 6.1 graphics the variation of numerical results with boundaries absorption coefficients for the different atmospheric attenuation values explained above.

As expected, estimated values of the reverberation time decrease when air absorption increases, showing an accurate behaviour of the model. Moreover, all predicted values using air absorption tends to the same values for 
surfaces absorption coefficients higher than 0.4 , because the influence of the atmospheric attenuation in the total room is lower than boundaries absorption.

It would be also interesting to obtain the relative error in order to analyse how significative are the differences when they are compared to the simulation without including the air absorption. Therefore, in the calculation of this error the results obtained from the simulation with no air absorption have been taken as a reference. These relative errors are shown in Fig. 6.2. It is observed that the relative error is higher than $5 \%$ in all cases. According to ISO/DIS 3382 [ISO, 2009], this percentage, $5 \%$ is the subjective limen for reverberation time. Since all these relative errors are above this limen, it means that differences will be subjectively noticeable. Therefore, the atmospheric attenuation has a considerable influence on the performance of the results and is modelled by the acoustic diffusion equation.

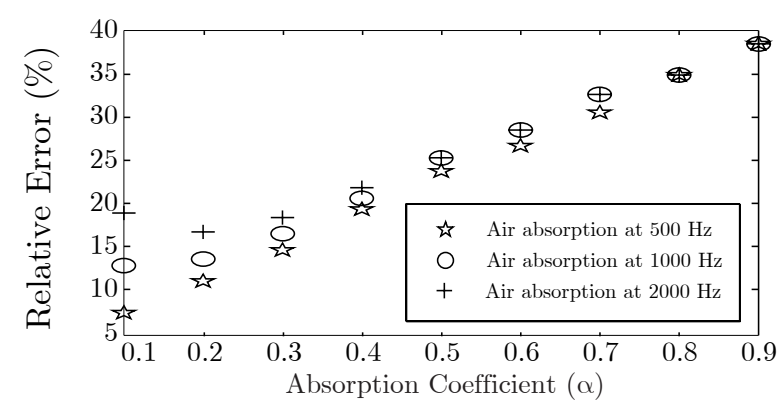

Figure 6.2. Relative error regarding to the no air absorption simulations for air absorption at $500 \mathrm{~Hz}(\star)$, air absorption at 1,000 $\mathrm{Hz}(\bigcirc)$ and air absorption at 2,000 $\mathrm{Hz}(+)$ predicted values.

In the following section, a comparison with other diffuse reflection geometrical models is presented. In these simulations, the air absorption is also incorporated. 


\subsubsection{Comparison with other diffuse reflection prediction models}

\subsubsection{Introduction}

Diffuse reflection, or scattering, describes the process of reflection from a diffuser or rough surface, and it is an important phenomenon in room acoustics [Cox et al., 2006]. In room acoustics, the reflection and scattering of sound waves at the boundaries strongly determines the behaviour within the enclosed space itself. Therefore, the accurate simulation of the acoustic characteristics of a boundary is an important part of any room acoustics prediction model, and in particular diffuse reflections at a boundary is one of the most important properties to model correctly for an accurate and perceptually natural result.

During the last years, the use of a diffusion equation has been applied to model sound propagation in room acoustics with perfectly diffuse walls [Picaut et al., 1997]. This section focuses on comparing predictions of the diffusion equation model, including the air absorption effect, with others geometrical diffuse reflection prediction models, such as radiosity and diffuse ray-tracing, for a large rectangular room configuration but with non-homogeneous absorption distribution [Navarro et al., 2010b].

The problems of diffuse reflection modelling in geometric models are well established. Hodgson [Hodgson, 1991] used a ray-tracing model taking into account this phenomenon through an equation which models the local effects of a perfectly diffuse reflection known as Lambert's law [Kuttruff, 4th edition, 2000], (see Sec. 2.2.7.1). One of his conclusions was that in simple empty rooms the effects of diffuse surface reflections are negligible in small proportionate rooms, while in large disproportionate rooms the effects can be considerable. Moreover, in the first round robin study of room acoustic models [Vorländer, 1995] it was concluded that the prediction models that include a method to model surface scattering perform significantly better than others. The lack of diffuse reflection modelling causes an overestimation of reverberation time [Hodgson, 1991]. This is specially 
true in rooms with an non-homogeneous distribution of the absorption on surfaces or in large rooms. There are many different methods for incorporating diffuse reflections into acoustic geometrical techniques [Dalenbäck et al., 1994]. However, despite the accuracy of these approaches, radiosity and ray-tracing, the overall computational cost and implicit limitations of geometrical methods has a non-trivial impact on the performance of such simulations.

Both acoustical radiosity and diffuse ray-tracing have been chosen as numerical reference models since both of them have shown a certain accuracy in prediction results [Hodgson and Nosal, 2006, Hodgson, 1990]. Radiosity is a geometrical sound field prediction method that assumes diffusely reflection boundaries by Lambert's law; furthermore, although ray-tracing used to be a specular reflection model, it could be modified to allow room surfaces to reflect incident energy diffusely. In this analysis the ray-tracing account for diffuse reflections with the ray re-direction method as suggested by Kuttruf [Kuttruff, 4th edition, 2000]. Finally, the diffusion equation models the diffuse reflections with an isotropic scattering function (see Sec. 4.5.2).

\subsubsection{Analysis}

For comparisons purposes, the prediction scenario has been the same configuration used by Nosal [Nosal, 2003] in her radiosity model investigations. In this paper, a small shoe-box office is used, which dimensions are $3.94 \mathrm{~m}$ wide, $5.36 \mathrm{~m}$ long and $2.71 \mathrm{~m}$ high. The real enclosure has a floor of vinyl tile on concrete, four walls of drywall on $100 \mathrm{~mm}$ studs, and a suspended acoustical-tile ceiling, which consist on different absorption coefficients detailed in Table 6.1. The FTCS numerical solution for the acoustic diffusion equation presented in Sec. 5.2.1 is used. In this case, the chosen configuration parameters are $\Delta t=1.10^{-5} \mathrm{~s}$ and $\Delta \nu=\Delta x=\Delta y=\Delta z=0.1 \mathrm{~m}$. The room is evaluated in octave-bands between $125 \mathrm{~Hz}$ and $8,000 \mathrm{~Hz}$ with two different absorption models with FTCS discretisation. The one using Sabine absorption factor $\left(A_{S}\right)$ is denominated FTCS Sabine (see Eq. (4.25)) and the other adopting modified absorption factor $\left(A_{M}\right)$, FTCS Modified 
(see Eq. (4.27)).

\begin{tabular}{lclllllll}
\hline \hline & \multicolumn{7}{c}{ Frequency band (Hz) } \\
\hline Office Room & Area & 125 & 250 & 500 & 1000 & 2000 & 4000 & 8000 \\
\hline Air- & - & 0.095 & 0.305 & 0.699 & 1.202 & 2.290 & 6.240 & 21.520 \\
absorption $\left(10^{-3} \mathrm{~m}^{-1}\right)$ & & & & & & & & \\
Ceiling & 21.12 & 0.200 & 0.140 & 0.160 & 0.170 & 0.270 & 0.300 & 0.300 \\
Floor & 21.12 & 0.010 & 0.010 & 0.010 & 0.010 & 0.010 & 0.020 & 0.010 \\
Walls & 50.41 & 0.060 & 0.050 & 0.030 & 0.020 & 0.030 & 0.040 & 0.030 \\
Room average & - & 0.081 & 0.061 & 0.055 & 0.052 & 0.080 & 0.095 & 0.087 \\
\hline \hline
\end{tabular}

Table 6.1. Air absorption exponents and surface absorption coefficients used in the predictions.

This room has been chosen for use in the comparison because it is small with relatively uniform geometry but non-uniform absorption distribution. The omnidirectional point source is placed at $(1.1,1.97,1.72) \mathrm{m}$ and receiver test position is $(3.25,1.97,0.9) \mathrm{m}$.

Using the time energy decay curves obtained from the predictions, the reverberation time (RT) (obtained from $\mathrm{T}_{30}$ ) and the early decay time (EDT) values were calculated using Schroeder's backward integration (see Sec. 2.3.3.1). Fig. 6.3 graphics the variation with frequency of diffusion equation numerical results estimated along with acoustical radiosity, diffuse ray-tracing and specular ray-tracing models extracted from Ref. [Nosal, 2003].

Diffusion equation, radiosity and diffuse ray-tracing generally give very similar predictions, but the ones from specular ray-tracing are found very different. In general, the diffuse reflection models show the same trend with a good accordance. Examining results in details, they show that FTCS Sabine has overestimated values predicted by radiosity model, while FTCS Modified slightly has underestimated the rest of results. Diffuse ray-tracing model has predicted the lowest values into diffuse reflection models group, 

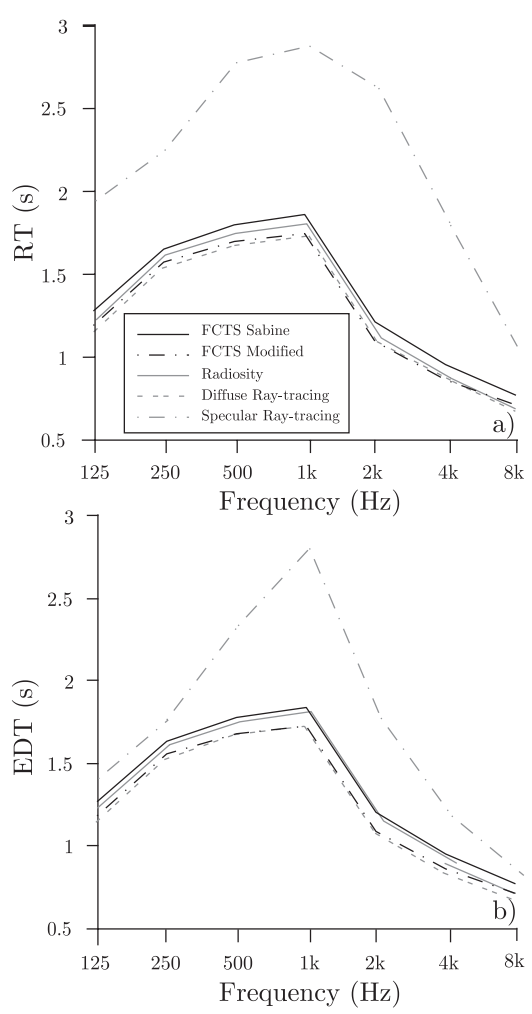

Figure 6.3. Reverberation time (RT) and Early decay time $(E D T)$ values as a function of frequency in the room under analysis.

showing results almost identical to modified diffusion model. Specular raytracing offers the highest calculated values in all frequencies, providing differences higher than $1 \mathrm{~s}$.

\subsubsection{Limitation in the temporal domain}

\subsubsection{Introduction}

As presented in Sec. 4.5.2, the diffusion equation model assumes that a sufficient number of reflection events occur to ensure that there are only 
small variations in the acoustic energy density and energy flow per meanfree path [Picaut et al., 1997], so-called directional and temporal broadening assumptions. This suggests, as discussed in Sec. 4.8 that the acoustic diffusion model is valid mainly for predicting the late part of reverberating sound fields in enclosed spaces.

These assumptions require a certain time delay after direct sound arrival in order to yield a sound energy distribution that can be compared against the solution of a diffusion equation model. Some authors provide tentative values of this time delay [Billon et al., 2006, Xiang et al., 2009]. Recently, a study of the validity of this argument has been made by Escolano, Navarro and Lopez [Escolano et al., 2010]. This study provides preliminary comparisons of several room-acoustic parameters, obtained from the impulse response of a geometrical acoustics approach and from the energy decay function of the diffusion model. The results are discussed in order to assist architectural acousticians in gaining a coherent understanding of the diffusion equation model.

\subsubsection{Analysis}

In the present work, the time at which the diffusion equation model becomes valid is estimated. Valeau et al. assert that this model becomes valid after one mean-free time [Valeau et al., 2006], but Xiang et al. state that at least two mean-free times are needed [Xiang et al., 2009]. Neither claim has yet been validated systematically.

The recent studies on the diffusion equation model have been mainly focused on evaluating the reverberation time (RT). This room-acoustic parameter is usually calculated using Schroeder's backward integration method [Xiang et al., 2009]. The standard method derives the reverberation time from a logarithmic slope regression between $-5 \mathrm{~dB}$ and $-35 \mathrm{~dB}$, so that it gives only very weak dependence of reverberation on the early reflections. However, this room-acoustic parameter does not seem adequate to estimate the time to be disregarded from the impulse response, and therefore the dependence 
on the first order reflections.

In this investigation, several room-acoustic parameters are evaluated after disregarding a certain initial duration from the impulse response to examine when they equal to those provided by the diffusion equation model. These investigations were performed using ray-tracing software with diffuse reflections.

Four further room-acoustic parameters are calculated: the early decay time (EDT), definition $\left(\mathrm{D}_{50}\right)$, clarity $\left(\mathrm{C}_{80}\right)$ and centre time (TS). These parameters may be approximately calculated from the diffusion equation model since they have a strong dependence on the energy of the first order reflections [Vorländer, 2008]. However, they are suitable for use as a reference when analyzing the decay slope function. The approach of these investigations is to disregard an appropriate initial interval from the raytracing impulse response, which is proportional to the time for sound to travel the mean-free path, $\lambda / c$, and to compare these room-acoustic parameters with those obtained from the diffusion equation model. To obtain these parameters, all of the integrals involved in the room-acoustic parameters calculation are taken up to time $t=n \lambda / c$, where $t=0$ is the arrival time of the direct sound and $n$ is the number of mean-free times to be disregarded. In other words, the parameter $\mathrm{D}_{50}$ is calculated by integrating over the early times from $t=n \lambda / c$ to $t=n \lambda / c+50 \mathrm{~ms}$.

The simulations were made in a shoe-box configuration $\left(10 \times 20 \times 10 \mathrm{~m}^{3}\right)$ same as previous Sec. 6.2.1, with an uniform absorption coefficient that increases monotonically from 0.1 to 0.9 at all interior surfaces. The diffusion equation model assumes that the overall absorption is not high [Valeau et al., 2006, Jing and Xiang, 2008], and the range of absorption coefficients has been chosen for illustrative purposes. The diffusion equation model is implemented by FTCS scheme using the same configuration parameters as in Sec. 6.2.1, and the room-acoustic parameters are calculated using two distinct boundary conditions: Eyring and modified boundary conditions.

For each configuration, ray-tracing-based simulations were conducted 
using EASE commercial software running with the AURA module, which incorporates complete diffusion and enables uniform scattering from the interior surfaces of the room [Vorländer, 1989]. Both simulations were performed at the same source - $(2.5,10,5) \mathrm{m}$ - and receiver positions - $(7.5,10,5)$, $(5,5,7.5)$ and $(5,15,2.5) \mathrm{m}$, and the results were evaluated at the octave frequency-band of $1 \mathrm{kHz}$. To facilitate comparison, this study disregards the direct sound and the early reflections from the ray-tracing impulse responses, and starts the calculation of the room-acoustic parameters after one to five mean-free times. In this specific room, the mean-free time corresponds to $23.5 \mathrm{~ms}$. In all configurations, the room-acoustic parameters were obtained by averaging the results at the different positions.

Figure 6.4 shows the room-acoustic parameters as functions of the absorption coefficients ranging between 0.1 and 0.9 . As expected, results of the reverberation time and the early decay time (see Fig. 6.4a) and b), respectively) do not show any particular dissimilarity when part of the ray-tracing impulse response is ignored. This shows that the decay of the Schroeder curves is linear, at least according to the reverberation time. Clear differences can be observed in the other room-acoustic parameters, however. The most significant are those obtained from the entire impulse response, since this includes the early reflections. If one or more mean-free times are disregarded, the agreement between ray-tracing and the diffusion equation model is considerably improved. Furthermore, in most cases, differences between the different boundary conditions used in the diffusion equation model are small for the clarity parameter $\mathrm{C}_{80}$; for $\mathrm{C}_{80}$, the modified boundary condition agrees well with most of the ray-tracing results, whereas the corresponding curve to the Eyring boundary condition starts to grow faster from $\alpha=0.6$, with considerable differences. These results further support the choice of the modified boundary condition for the diffusion equation model. Although the results are improved, there are some oscillations around the diffusion equation model solution, and the decision of how many mean-free times to exclude becomes more difficult. 

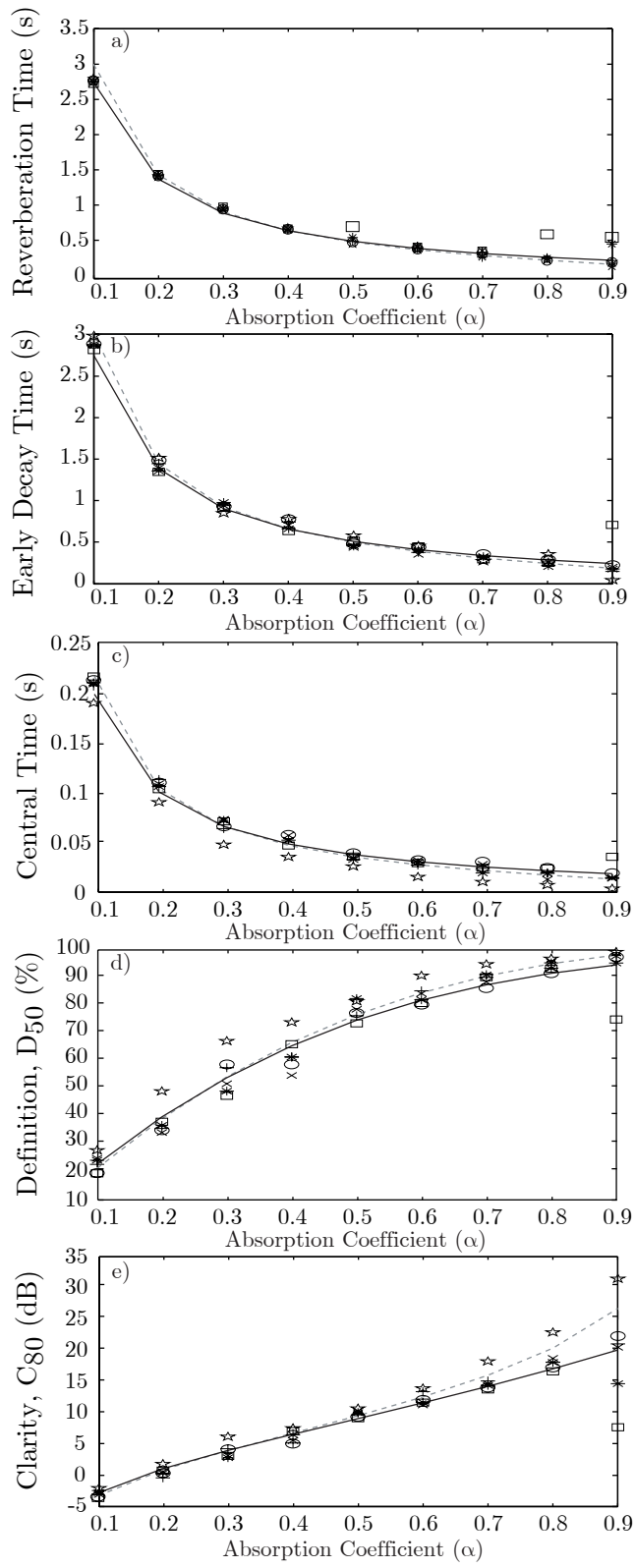

$\begin{array}{llll} & \text { Diffusion Model Eyring } & + & \text { Ray-tracing }-2 \lambda / c \mathrm{~s} \\ - \text { Diffusion Model Modified } & \times & \text { Ray-tracing }-3 \lambda / c \mathrm{~s} \\ \text { Ray-tracing }-0 \lambda / c \mathrm{~s} & * & \text { Ray-tracing }-4 \lambda / c \mathrm{~s} \\ & \text { Ray-tracing }-1 \lambda / c \mathrm{~s} & \square & \text { Ray-tracing }-5 \lambda / c \mathrm{~s}\end{array}$

Figure 6.4. Comparison of room-acoustic parameters: (a) reverberation time, (b) early decay time, (c) centre time, (d) speech definition and (e) clarity, where the diffusion equation model is compared to the ray-tracing after disregarding the early energy of 0 to 5 mean-free times, as a function of the overall absorption coefficient. 
Moreover, the results of the ray-tracing simulations with the results of applying the diffusion model equation using the modified boundary conditions are compared. These differences are also evaluated in terms of just noticeable differences. According to ISO/DIS 3382 [ISO, 2009], the subjective threshold for the reverberation time, the early decay time and the definition index is $5 \%$, whereas for the clarity index it is required to be $1 \mathrm{~dB}$ and the centre time is $10 \mathrm{~ms}$. Table 6.2 lists the average difference and its corresponding standard deviation in each situation. The mean has been computed as a function of the absorption coefficients in a range from 0.1 to 0.9. As expected, the greatest average differences are found using the entire ray-tracing impulse response, whereas if one mean-free time is disregarded, the average difference approaches the subjective limens. However, the average difference in these terms becomes greater than the subjective threshold for the early decay times, ranging between $7.7 \%$ and $11.2 \%$ when the early energy of one to three mean-free times are disregarded; the difference for clarity is approximately $1 \mathrm{~dB}$. These results indicate that at least one mean-free time should be disregarded, as proposed by Valeau et al. [Valeau et al., 2006].

\begin{tabular}{c|ccccc} 
& $\mathrm{RT}(\%)$ & EDT $(\%)$ & $\mathrm{D}_{50}(\%)$ & $\mathrm{C}_{80}(\mathrm{~dB})$ & $\mathrm{TS}(\mathrm{ms})$ \\
\hline $0 \mathrm{mft}$ & $5.2(4.2)$ & $20.3(23.5)$ & $13.8(7.9)$ & $3.4(3.5)$ & $13.4(2.9)$ \\
$1 \mathrm{mft}$ & $5.0(5.4)$ & $7.7(4.9)$ & $6.2(5.3)$ & $1.0(0.6)$ & $4.3(5.3)$ \\
$2 \mathrm{mft}$ & $4.6(5.4)$ & $11.7(10.2)$ & $4.6(2.9)$ & $1.2(0.5)$ & $4.2(3.8)$ \\
$3 \mathrm{mft}$ & $8.3(9.3)$ & $11.2(7.7)$ & $6.9(5.3)$ & $0.9(0.6)$ & $5.3(3.2)$ \\
$4 \mathrm{mft}$ & $16.2(30.5)$ & $6.4(8.3)$ & $5.3(3.7)$ & $1.1(1.7)$ & $4.4(2.9)$ \\
$5 \mathrm{mft}$ & $37.6(55.1)$ & $29.9(63.0)$ & $6.8(7.3)$ & $1.8(4.0)$ & $5.4(7.0)$ \\
\hline
\end{tabular}

Table 6.2. Average difference and their corresponding standard deviation (indicated inside the brackets) when the early energy of zero to five mean-free times ( $m f t$ ) is disregarded. All the data are computed as a function of the absorption coeffcient, ranged to 0.1 to 0.9 . 
However, the diffusion equation model assumes that the scattered or reflected energy is greater than that absorbed, so that predictions for surface materials having low-to-medium absorption coefficients should be accurate. For this reason a new averaging process has been undertaken, this time as a function of absorption coefficients varying between 0.1 and 0.5 . Table 6.3 lists these results. The average error is reduced in all cases, especially for the clarity index $\mathrm{C}_{80}$, for which the results are very close to $0.5 \mathrm{~dB}$. Major differences in the definition parameter $\mathrm{D}_{50}$ are concentrated in the absorption coefficient range between 0.2 and 0.4 (see Fig. 6.4d)); however, when the early energy of two mean-free times is disregarded, the average difference becomes nearly the same as the previous scenario. In the range of absorption coefficients between 0.1 and 0.5 it is therefore affirmed that the average difference is minor in most of the room-acoustic parameters.

Table 6.3 lists that at least three room acoustic parameters - the reverberation time, the definition index and the centre time - have lowest difference when the early energy of two mean-free times is disregarded from the ray-tracing impulse response. The early decay time and the clarity index do not have their lowest difference in this situation, but their differences when the early energy of one or three mean-free times is excluded can be considered negligible. In consequence, it is indeed necessary to disregard at least the early energy of two mean-free times from the ray-tracing impulse response, consistent with recent works [Xiang et al., 2009].

The point beyond which the diffusion equation model is valid is related to the mixing time. The latter is defined as the time at which the transition occurs from early reflections to late reverberation. Some authors suggest that it is equal to three mean-free times [Blesser, 2001]. This has to be considered as an upper limit, however, because the transition in the impulse response is highly dependent on the geometry and on the diffusion properties of boundaries [Polack, 1992]. The diffusion equation model assumes that the scattering density is high, and that reflection of energy is dominant over absorption, so that after numerous diffuse reflections the energy density 


\begin{tabular}{l|ccccc} 
& RT (\%) & EDT (\%) & D $_{50}(\%)$ & $\mathrm{C}_{80}(\mathrm{~dB})$ & $\mathrm{TS}(\mathrm{ms})$ \\
\hline $0 \mathrm{mft}$ & $3.9(1.9)$ & $11.3(5.2)$ & $18.7(7.0)$ & $1.3(0.6)$ & $12.3(3.6)$ \\
$1 \mathrm{mft}$ & $3.1(2.1)$ & $8.1(5.8)$ & $9.9(4.1)$ & $0.6(0.5)$ & $6.6(6.2)$ \\
$2 \mathrm{mft}$ & $2.9(2.3)$ & $7.7(3.4)$ & $5.2(4.0)$ & $0.8(0.6)$ & $5.7(4.7)$ \\
$3 \mathrm{mft}$ & $3.8(2.2)$ & $7.0(4.8)$ & $9.9(5.4)$ & $0.7(0.5)$ & $7.0(2.7)$ \\
$4 \mathrm{mft}$ & $5.2(3.9)$ & $4.6(3.9)$ & $8.1(1.7)$ & $0.6(0.5)$ & $5.9(3.0)$ \\
$5 \mathrm{mft}$ & $12.0(17.6)$ & $3.0(0.7)$ & $6.7(6.3)$ & $0.5(0.3)$ & $5.7(6.9)$ \\
\hline
\end{tabular}

Table 6.3. Average differences and their corresponding standard deviation (indicated inside the brackets) when the early energy of zero to five mean-free times ( $m f t$ ) is disregarded. All the data are computed as a function of the absorption coeffcient, ranged to 0.1 to 0.5 .

becomes nearly isotropic. This does not mean, however, that a perfectly diffuse sound field prevails in the diffusion equation model from the beginning. Also, the late reverberation assumes that the acoustical energy density is distributed uniformly across a room with homogeneous dimensions.

These findings suggest that the diffusion equation models not only the late reverberation but also a certain fraction of early reflections (approximately one mean-free time) prior to the time at which mixing occurs. A more detailed analysis and further experiments should be conducted in order to provide sharper conclusions [Defrance and Polack, 2010].

In next section, further investigations will be performed, in which the diffusion model is applied in a more real situation such as a complex shape room, and the numerical results should be compared with other geometrical methods as well as with measured data. 


\subsection{Simulations in a complex shape room}

After that the applicability of the diffusion equation model in room acoustics simulations for rectangular rooms has been investigated in the previous section, it is of interest to evaluate the model in a real room environment to study its performance and limitations.

\subsubsection{Introduction}

In Chap. 4 a model named acoustic radiative transfer equation has been proposed as a general theory to expand geometrical room acoustics modelling algorithms. It has been shown that this room acoustics modelling technique establishes the basis of two recently proposed algorithms, the acoustic diffusion equation model and the room-acoustic rendering equation.

This section presents some comparisons of room-acoustic parameters insitu measurements with prediction values from the acoustic diffusion equation, the room-acoustic rendering equation models and other geometrical models in a real complex shape room in order to clarify advantages and limitations of the diffusion equation model.

In this case, the model used was a room for which there is available public acoustic measurement data, in addition to the data produced by other room acoustics prediction software. The enclosure of the third Round Robin on room acoustical simulation, the music recording studio of the $\mathrm{PTB}, 400 \mathrm{~m}^{3}$ volume, has been chosen because is appropriate to evaluate the models in real rooms environments [Bork, 2005a,b]. In this evaluation, only Phase 2 geometry consisting of 68 planes and 138 corners with curtains open and closed configurations were modelled because it has enough details to produce unique responses at different receiver and source positions. The simulations were run separately for each frequency band between $125 \mathrm{~Hz}$ and $4 \mathrm{kHz}$ including air absorption. The materials properties are given as absorption and scattering coefficients for the six octave bands. The length of the response was $1.0 \mathrm{~s}$. In Fig. 6.5 the room model before discretisation 
in cells is shown. Each surface's color indicates a different material.

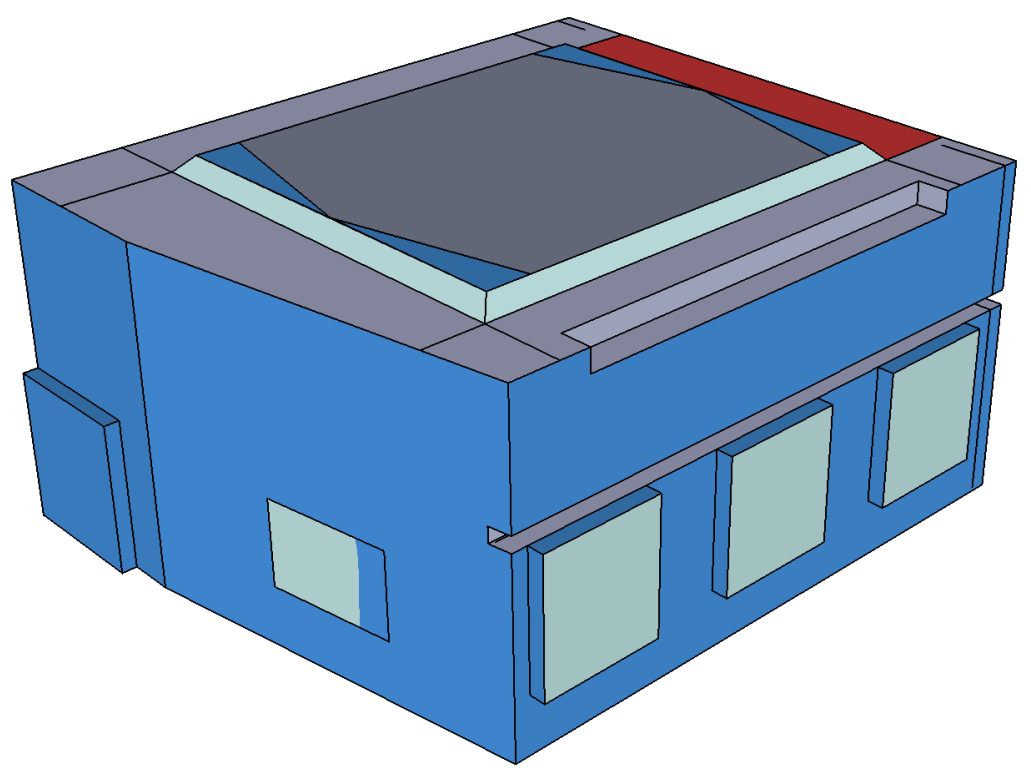

Figure 6.5. 3-D room model before discretisation in cells.

The algorithm for the room-acoustic rendering equation follows the structure of the progressive radiosity algorithm [Cohen et al., 1988] and simulations were carried out with a detail of the model consisting of 378 patches, using angular resolution of $30^{\circ}$ for both azimuth and elevation angles, and a time resolution of $1 \mathrm{~ms}$. The numerical solution for the acoustic diffusion equation model is based on DF scheme (see Sec 5.2.3). A homogeneous spatial resolution of $\Delta \nu=0.2 \mathrm{~m}$ and a time resolution of $0.125 \mathrm{~ms}$ $\left(\Delta t=8,000 \mathrm{~s}^{-1}\right)$ were the simulations configuration parameters selected. Despite the materials characteristics are given as absorption and scattering coefficients in Ref. [Bork, 2005b], only the absorption coefficients for each octave band were used in the diffusion equation predictions, because total diffuse reflection is assumed. In Fig. 6.6 the room model after discretisation 
in cells is shown.

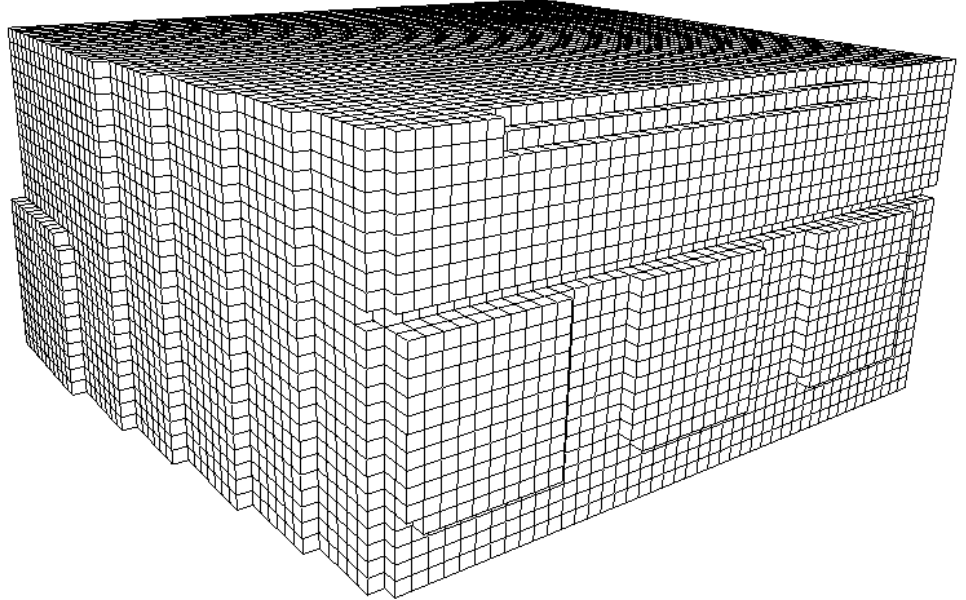

Figure 6.6. 3-D room model after discretisation in cells.

\subsubsection{Analysis}

The temporal distribution of energy has been studied using room-acoustic parameters predictions. Moreover, the spatial variation behavior of these parameters has been evaluated obtaining results for combinations of two source and three receiver positions. These results allow to observe the advantages and disadvantages of these methods. Estimated data with the acoustic diffusion equation algorithm are compared with the measured data of the actual room and with the mean averaged value of predicted data from the other participants in the Round Robin test [Bork, 2005b]. Five acoustic parameters are compared, namely reverberation time RT (from $\mathrm{T}_{30}$ ), early decay time EDT, definition $\mathrm{D}_{50}$, clarity $\mathrm{C}_{80}$, and center time 
TS. The data in tables and in the figures correspond to that shown by Bork for the measurements and the mean value of participants in the round robin test [Bork, 2005b], by Siltanen et al. [Siltanen et al., 2007a] for the room-acoustic rendering equation predictions.

In order to give a good insight about the positional dependence, the Tables 6.4-6.8 present averages values of the four middle frequencies with curtains open for varying positions. Figures 6.7-6.12 show the frequency dependence of some predicted values in positions of interest.

\begin{tabular}{|c|c|c|c|c|c|c|c|}
\hline Sr./Rc. & Meas. & $\begin{array}{c}\text { Sim. } \\
\text { OM } \\
(\mathrm{s})\end{array}$ & $\begin{array}{c}\text { Sim. } \\
\text { RE } \\
\text { (s) }\end{array}$ & $\begin{array}{c}\text { Sim. } \\
\text { DE } \\
(\mathrm{s})\end{array}$ & $\begin{array}{l}\text { Err. } \\
\text { OM } \\
(\%)\end{array}$ & $\begin{array}{l}\text { Err. } \\
\text { RE } \\
(\%)\end{array}$ & $\begin{array}{c}\text { Err. } \\
\text { DE } \\
(\%)\end{array}$ \\
\hline $\mathrm{S} 1 / \mathrm{R} 1$ & 1.12 & 1.12 & 1.11 & 1.11 & 0.1 & 0.9 & 0.9 \\
\hline $\mathrm{S} 1 / \mathrm{R} 2$ & 1.15 & 1.11 & 1.11 & 1.11 & 3.2 & 3.5 & 3.5 \\
\hline S1/R3 & 1.13 & 1.13 & 1.11 & 1.11 & 0.1 & 1.8 & 1.8 \\
\hline $\mathrm{S} 2 / \mathrm{R} 1$ & 1.13 & 1.11 & 1.11 & 1.11 & 2.0 & 1.8 & 1.8 \\
\hline $\mathrm{S} 2 / \mathrm{R} 2$ & 1.13 & 1.13 & 1.11 & 1.11 & 0.0 & 1.8 & 1.8 \\
\hline S2/R3 & 1.11 & 1.11 & 1.11 & 1.11 & 0.1 & 0.0 & 0.0 \\
\hline
\end{tabular}

Table 6.4. The measured, other models (OM), rendering equation model ( $R E)$, and diffusion equation model ( $D E)$ simulated reverberation time (RT) values averaged for the $250 \mathrm{~Hz}$, $500 \mathrm{~Hz}, 1 \mathrm{kHz}$, and $2 \mathrm{kHz}$ octave bands. Relative error values between $O M$ and measured, $R E$ and measured, and $D E$ and measured for the six combinations of source and receiver positions are given.

The values estimated of RT in Table 6.4 are very good for all methods remaining below the subjective difference limen (5\%) specified in ISO 3382. To study the frequency dependence Fig. 6.7 shows the values for each octave band at position S2/R2. Comparing with the measurements, values are higher at low frequencies and lower at higher frequencies with curtains open curtains. However in closed curtains configuration all predicted values 


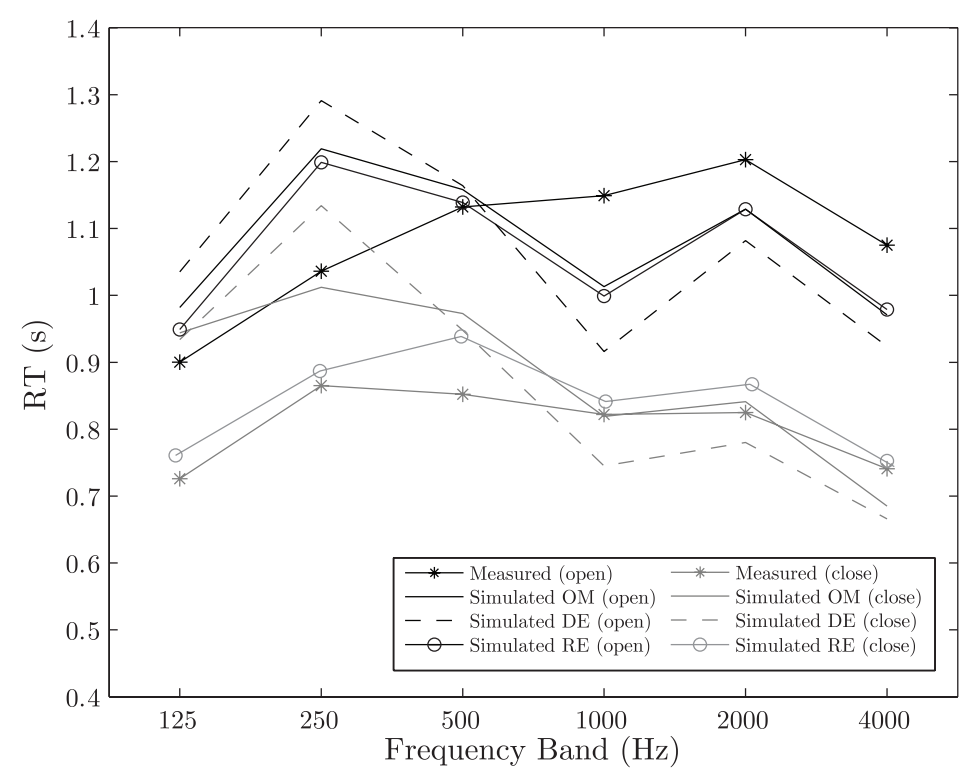

Figure 6.7. RT values at position S2/R2.

in the high frequency-band are considerable near the measured data. Moreover, the acoustic diffusion model predictions present a similar tendency and values in the frequency response to those shown for other prediction software and for the rendering equation, resulting in a mean percentage difference of $5 \%$ in both configurations.

The values presented in Table 6.5 for the averaged EDT also follow the measured values quite accurately in all methods. Except S2/R2 position for render equation algorithm and the farthest position S1/R3 for diffusion equation algorithm, all other values are below the subjective difference limen (5\%). More conclusions can be obtained from Fig. 6.8, that shows the values for each position in the $1 \mathrm{kHz}$ band. The variation of predicted values in the rendering equation algorithm is larger than in RT. Also, the positional dependence in the EDT is shown, both rendering equation and other simulations models data, mainly in curtains open configuration, though there are some deviations from measurements values around $10 \%$ of mean 


\begin{tabular}{cccccccc}
\hline \hline EDT & Meas. & Sim. & Sim. & Sim. & Err. & Err. & Err. \\
& & OM & RE & DE & OM & RE & DE \\
Sr./Rc. & $(\mathrm{s})$ & $(\mathrm{s})$ & $(\mathrm{s})$ & $(\mathrm{s})$ & $(\%)$ & $(\%)$ & $(\%)$ \\
\hline S1/R1 & 1.16 & 1.12 & 1.15 & 1.11 & 3.8 & 0.9 & 4.3 \\
S1/R2 & 1.07 & 1.12 & 1.08 & 1.11 & 4.4 & 0.9 & 3.7 \\
S1/R3 & 1.20 & 1.13 & 1.19 & 1.12 & 5.5 & 0.8 & 6.7 \\
S2/R1 & 1.11 & 1.08 & 1.07 & 1.11 & 2.4 & 3.6 & 0.0 \\
S2/R2 & 1.12 & 1.13 & 1.19 & 1.11 & 0.9 & 6.2 & 0.9 \\
S2/R3 & 1.11 & 1.10 & 1.10 & 1.11 & 0.8 & 0.9 & 0.0 \\
\hline \hline
\end{tabular}

Table 6.5. The measured, other models (OM), rendering equation model ( $R E)$, and diffusion equation model ( $D E)$ simulated early decay time (EDT) values averaged for the $250 \mathrm{~Hz}$, $500 \mathrm{~Hz}, 1 \mathrm{kHz}$, and $2 \mathrm{kHz}$ octave bands. Relative error values between $O M$ and measured, $R E$ and measured, and $D E$ and measured for the six combinations of source and receiver positions are given.

error. However, the diffusion equation algorithm shows lower variability suggesting that it does not simulate the positional dependence properly.

The direct sound and the early reflections mainly determine the values of $\mathrm{D}_{50}$ and $\mathrm{C}_{80}$. As expressed in Table 6.6, averaged values are mostly near the measured data. As expected, results are more inaccurate using the diffusion equation model when the distance from the source to the receiver are small corresponding to S1/R2, S2/R1, and S2/R3 positions values with a difference over the subjective limen. This becomes more clear observing the positional dependence in Fig. 6.9 for measured and predicted data. Although the variation in individual values for the diffusion equation model are smaller $(2.6 \%)$ than the values for the rendering equation or other simulation methods ( $20 \%$ and $13 \%$ respectively), it can be seen that the predicted values with the diffusion equation tend to the mean value of all positions. Fig. 6.10 shows noticeable differences with the measurements 


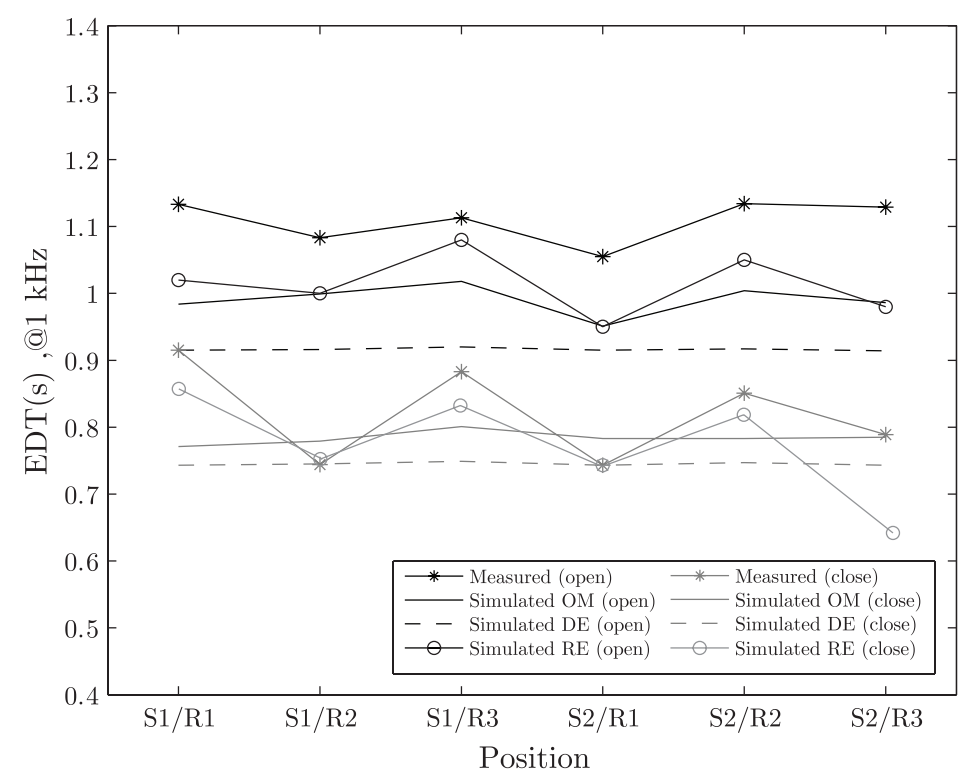

Figure 6.8. EDT values in the $1 \mathrm{kHz}$ octave band.

in the lowest octave band data due to its implicit limitations, nevertheless compared to the values given by other room acoustic geometrical software the results by diffusion equation are similar with a mean percentage difference around $8 \%$ in open curtains configuration and around $5 \%$ in closed curtains configuration.

The $\mathrm{C}_{80}$ values presented in Table 6.7 show similar behavior as $\mathrm{D}_{50}$ in the positional dependence. However, the shape of the curve for each frequency band in Fig. 6.11 is similar for all prediction models. It can be concluded that the early reflections are not properly modelled by the acoustic diffusion equation method. However, it is important to note that the predicted data gives a good estimation of the mean value, especially for large distances from the source and specular reflectance surfaces.

To calculate the centre time the complete impulse response is taken into account, hence later reflections are also evaluated. As it could be seen in 


\begin{tabular}{|c|c|c|c|c|c|c|c|}
\hline Sr./Rc. & Meas. & $\begin{array}{c}\text { Sim. } \\
\text { OM } \\
(\mathrm{s})\end{array}$ & $\begin{array}{c}\text { Sim. } \\
\text { RE } \\
(\mathrm{s})\end{array}$ & $\begin{array}{c}\text { Sim. } \\
\text { DE } \\
(\mathrm{s})\end{array}$ & $\begin{array}{l}\text { Err. } \\
\text { OM } \\
(\%)\end{array}$ & $\begin{array}{c}\text { Err. } \\
\text { RE } \\
(\%)\end{array}$ & $\begin{array}{c}\text { Err. } \\
\text { DE } \\
(\%)\end{array}$ \\
\hline $\mathrm{S} 1 / \mathrm{R} 1$ & 49.19 & 46.70 & 48.94 & 46.69 & 2.5 & 0.3 & 2.5 \\
\hline $\mathrm{S} 1 / \mathrm{R} 2$ & 56.61 & 49.75 & 54.68 & 46.70 & 6.9 & 1.9 & 9.9 \\
\hline $\mathrm{S} 1 / \mathrm{R} 3$ & 46.13 & 45.24 & 45.04 & 45.85 & 0.9 & 1.1 & 0.3 \\
\hline $\mathrm{S} 2 / \mathrm{R} 1$ & 53.87 & 52.23 & 55.53 & 46.98 & 1.6 & 1.7 & 6.9 \\
\hline $\mathrm{S} 2 / \mathrm{R} 2$ & 43.49 & 46.64 & 44.33 & 46.34 & 3.1 & 0.8 & 2.9 \\
\hline S2/R3 & 53.23 & 51.98 & 51.22 & 47.09 & 1.3 & 2.0 & 6.1 \\
\hline
\end{tabular}

Table 6.6. The measured, other models (OM), rendering equation model ( $R E)$, and diffusion equation model ( $D E)$ simulated definition index $\left(D_{50}\right)$ values averaged for the $250 \mathrm{~Hz}$, $500 \mathrm{~Hz}, 1 \mathrm{kHz}$, and $2 \mathrm{kHz}$ octave bands. Error values between $O M$ and measured, $R E$ and measured, and DE and measured for the six combinations of source and receiver positions are given.

Table 6.8, the predicted values are a little higher than the measured values in general, nevertheless almost all TS values are within the subjective difference limen (10 ms). Again, as expected the diffusion equation algorithm shows difficulties in modelling the positional dependence. However, since the predicted data for position where the reverberant field is predominant are quite accurate, it is reasonable to believe that the energy of the late part of the response is properly constructed. Frequency dependence can be seen in Fig. 6.8 at position S1/R1 repeating the behavior observed in other parameters offering a better performance in high frequencies.

\subsection{Summary}

The interest on performing accurate solutions, as well as finding computationally efficient algorithm, is a challenging issue in the field of room 


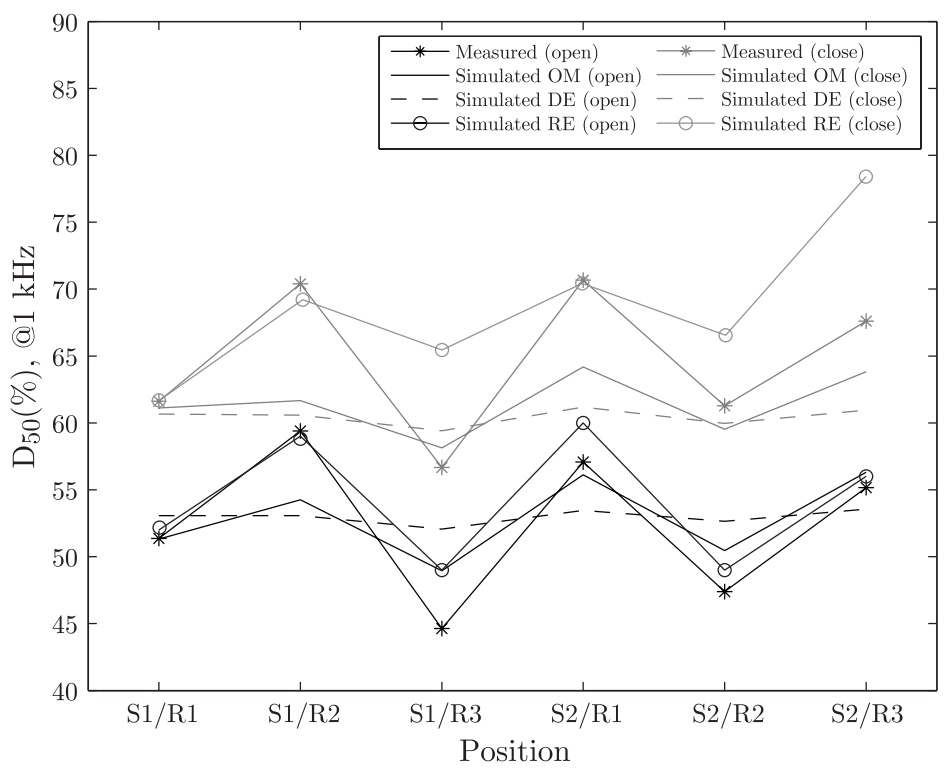

Figure 6.9. $D_{50}$ values in the $1 \mathrm{kHz}$ octave band.

acoustics simulation.

In this chapter, a study of the applicability of the acoustic diffusion equation for room acoustics simulations is done. Some comparisons with geometrical models and measured data to evaluate the theoretical advantages and limitations of the diffusion equation model in rooms with both rectangular and complex shapes are carried out.

The incorporation of the atmospheric attenuation in the governing equation has been firstly investigated. The predictions shows a correct air absorption dependence in terms of reverberation time. It is observed that the atmospheric attenuation has a remarkable influence on predictions performance when large rooms are simulated.

Then, the diffusion equation predictions are compared with the acoustical radiosity predictions, as a total diffuse reference method, and ray-tracing predictions (both completely specular and completely diffuse reflections). 


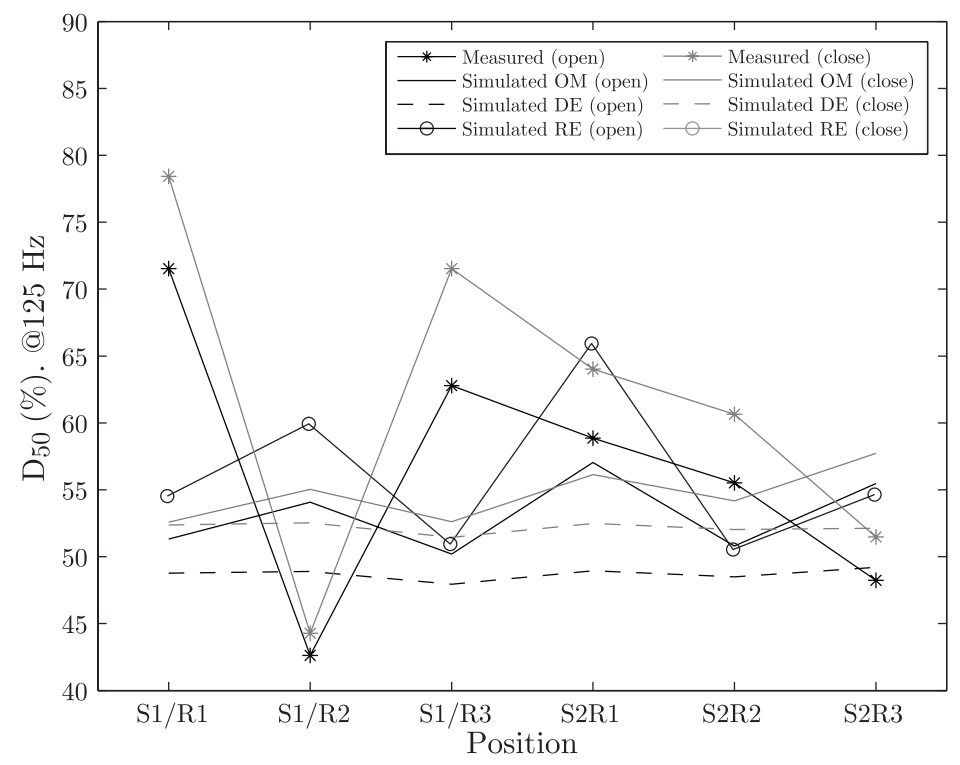

Figure 6.10. $D_{50}$ values in the $125 \mathrm{~Hz}$ octave band.

Predictions obtained with two different absorption models of the diffusion equation agreed closely with other diffuse reflections models, but were quite different from specular ray-tracing as expected. In general, the results of this work show that diffusion equation model could be used for predicting room sound fields with some accuracy, at least compared with results from other well-known energy-based approaches that assume diffuse reflections in this type of rectangular rooms.

In the technical literature, some authors have been suggested that the diffusion equation model is mainly accurate for predicting the late part of the decay process. However, no systematic studies have yet been published of the number of discrete early reflections, measured in terms of the meanfree time, after which the diffusion equation model can be considered valid. Comparisons with a ray-tracing model with complete diffusing boundaries show that there is good agreement between these methods whenever the first order reflections are excluded from the impulse response of the ray-tracing 


\begin{tabular}{|c|c|c|c|c|c|c|c|}
\hline Sr./Rc. & Meas. & $\begin{array}{c}\text { Sim. } \\
\text { OM } \\
\text { (s) }\end{array}$ & $\begin{array}{c}\text { Sim. } \\
\text { RE } \\
(\mathrm{s})\end{array}$ & $\begin{array}{c}\text { Sim. } \\
\text { DE } \\
(\mathrm{s})\end{array}$ & $\begin{array}{l}\text { Err. } \\
\text { OM } \\
(\%)\end{array}$ & $\begin{array}{c}\text { Err. } \\
\text { RE } \\
(\%)\end{array}$ & $\begin{array}{c}\text { Err. } \\
\text { DE } \\
(\%)\end{array}$ \\
\hline $\mathrm{S} 1 / \mathrm{R} 1$ & 2.85 & 2.38 & 2.59 & 2.39 & 0.5 & 0.3 & 0.5 \\
\hline $\mathrm{S} 1 / \mathrm{R} 2$ & 3.51 & 2.80 & 3.55 & 2.38 & 0.7 & 0.0 & 1.1 \\
\hline $\mathrm{S} 1 / \mathrm{R} 3$ & 1.90 & 2.13 & 2.16 & 2.27 & 0.2 & 0.3 & 0.4 \\
\hline $\mathrm{S} 2 / \mathrm{R} 1$ & 3.09 & 3.26 & 3.51 & 2.42 & 0.2 & 0.4 & 0.7 \\
\hline $\mathrm{S} 2 / \mathrm{R} 2$ & 1.94 & 2.24 & 2.20 & 2.34 & 0.3 & 0.3 & 0.4 \\
\hline $\mathrm{S} 2 / \mathrm{R} 3$ & 3.08 & 3.10 & 3.10 & 2.44 & 0.0 & 0.0 & 0.6 \\
\hline
\end{tabular}

Table 6.7. The measured, other models (OM), rendering equation model ( $R E)$, and diffusion equation model ( $D E)$ simulated clarity index $\left(C_{80}\right)$ values averaged for the $250 \mathrm{~Hz}, 500$ $\mathrm{Hz}, 1 \mathrm{kHz}$, and $2 \mathrm{kHz}$ octave bands. Error values between $\mathrm{OM}$ and measured, RE and measured, and DE and measured for the six combinations of source and receiver positions are given.

simulations. Several different room-acoustic parameters are employed, after disregarding the early energy of several mean-free times, to estimate the time at which the diffusion equation model becomes accurate. This study confirms the recommendations of recent studies that the diffusion equation model is valid after two mean-free times. Furthermore, the modified boundary condition agrees better that the Eyring boundary conditions with most of the ray-tracing results, especially for high absorption values. These results support the choice of the modified boundary condition for the diffusion equation model.

More investigations to evaluate the diffusion equation model accuracy using real measurements and predicted values from other simulations methods that handle specular and diffuse reflections in a complex shape room are done. The model used is the enclosure of the third round robin on room acoustical simulation for which there is available public acoustic measurement data, in addition to the data produced by other room acoustics 


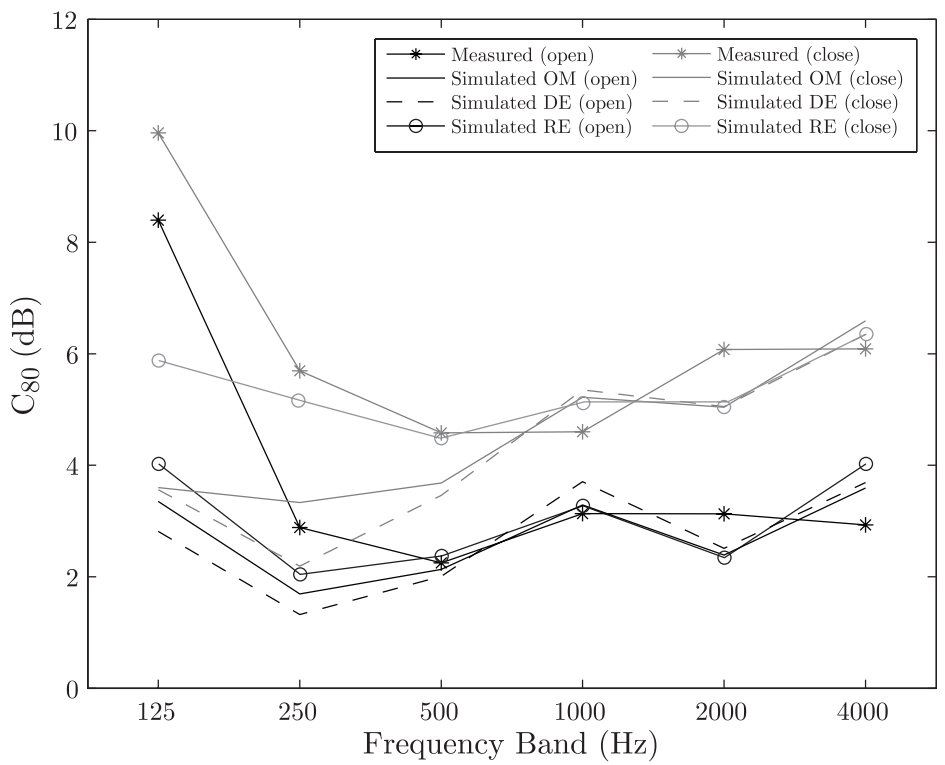

Figure 6.11. $C_{80}$ values at position S2/R1.

prediction software.

Special attention is paid on the comparison between the acoustic diffusion equation and the room-acoustic rendering equation. Since both models comes from the acoustic radiative transfer model, it is necessary a qualitative comparison. In this study, some preliminary tests comparing both models over a complex scenario of a single room, including the systematic acoustic measurements over the original room are presented. In general, both models provide good estimation for the room-acoustic parameters evaluated, at least as reliable as most of the geometrical methods used today. In particular, the acoustic diffusion equation model have worse accuracy in some conditions due to its theoretical assumptions. It has some difficulties when values for positions near the source or the boundaries, mainly affected by the early reflections and the direct sound, are predicted. Accordingly, it can be concluded that the positional dependence of the parameters is not properly modelled by the acoustic diffusion equation model. However, based 


\begin{tabular}{cccccccc}
\hline \hline TS & Meas. & $\begin{array}{c}\text { Sim. } \\
\text { OM }\end{array}$ & $\begin{array}{c}\text { Sim. } \\
\text { RE }\end{array}$ & $\begin{array}{c}\text { Sim. } \\
\text { DE } \\
(\mathrm{s})\end{array}$ & $\begin{array}{c}\text { Err. } \\
\text { OM } \\
(\%)\end{array}$ & $\begin{array}{c}\text { Err. } \\
\text { RE } \\
(\%)\end{array}$ & $\begin{array}{c}\text { Err. } \\
\text { DE } \\
\text { Sr./Rc. }\end{array}$ \\
\hline S1/R1 & 78.79 & 80.14 & 83.12 & 80.39 & 1.4 & 4.3 & 1.6 \\
S1/R2 & 68.19 & 76.01 & 72.73 & 80.34 & 7.8 & 4.5 & 12.2 \\
S1/R3 & 84.52 & 83.14 & 90.56 & 81.47 & 1.4 & 6.0 & 3.0 \\
S2/R1 & 71.25 & 71.65 & 72.72 & 79.98 & 0.4 & 1.5 & 8.7 \\
S2/R2 & 82.69 & 81.62 & 90.81 & 80.83 & 1.1 & 8.1 & 1.9 \\
S2/R3 & 71.68 & 77.72 & 77.02 & 79.85 & 1.0 & 5.3 & 8.2 \\
\hline \hline
\end{tabular}

Table 6.8. The measured, other models (OM), rendering equation model ( $R E)$, and diffusion equation model ( $D E)$ simulated centre time (TS) values averaged for the $250 \mathrm{~Hz}, 500 \mathrm{~Hz}$, $1 \mathrm{kHz}$, and $2 \mathrm{kHz}$ octave bands. Error values between $O M$ and measured, RE and measured, and DE and measured for the six combinations of source and receiver positions are given.

on these results it is reasonable to contemplate that the sound energy of the late part of the response is properly predicted. These features together with a lower computational cost indicate that the diffusion equation model can be useful for specific applications, e.g. first phase of a room acoustic design, taking advantage of a more accurate prediction of the rendering equation in the last phase of the design. 


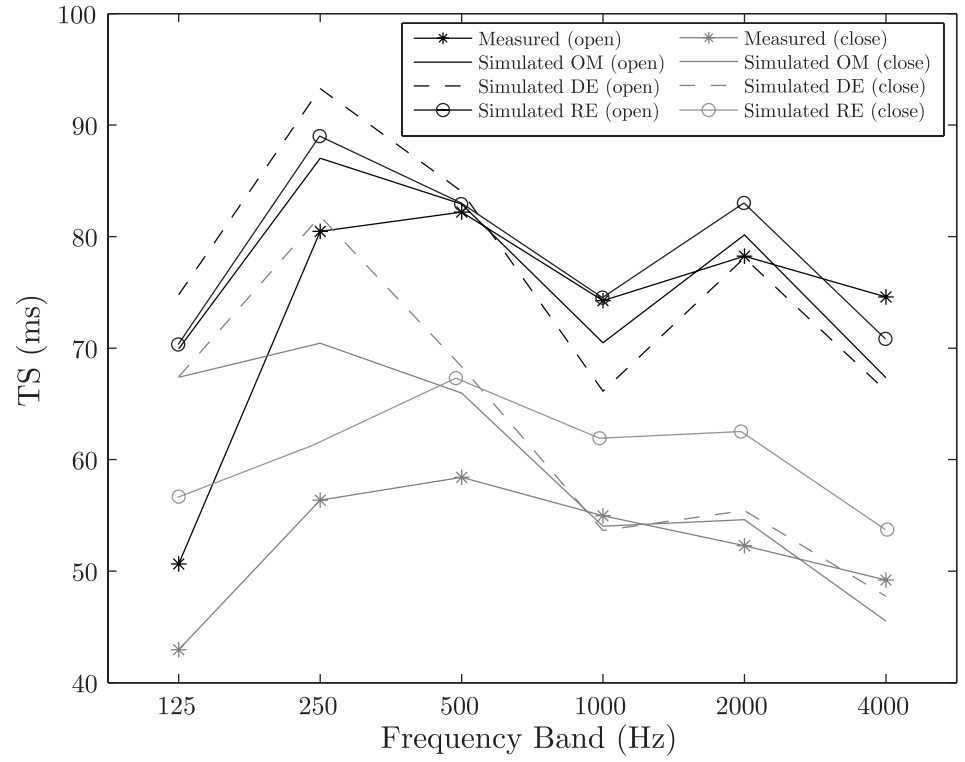

Figure 6.12. TS values at position $S 2 / R 1$. 


\section{Conclusions and Future Research Lines}

As concluding remarks, a brief description of the conclusions and contributions of this thesis is presented, referencing the journal articles and conference papers that have been published from this work, as well. Finally, some proposed guidelines for future research lines are indicated (some of them have already started).

\subsection{Summary and conclusions}

The sound wave propagation within rooms is a complex phenomenon where a lot of variables have to be taken into account to be properly simulated. Some simplifications should be applied to this room acoustics theory in order to perform computer simulations. Depending in the approximation of the sound field distribution several theories are available, see Chap. 2. The computer simulations of acoustic enclosures have been an active research area resulting in some commercial software that are used by engineers and architects, as well as researchers. 
In recent years, simulation of acoustic enclosures has been an active research area, resulting in various software applications. Most of these computer programs are based on a mathematical approach using a simplified model of wave propagation in a particular configuration As seen in Chap. 3, depending on which approach is selected to perform the simulation, a number of advantages and drawbacks exists.

In this thesis, the applicability of a diffusion equation model is justified since it is a promising simulation method for general purpose room acoustics design that incorporates diffuse reflections and offers low computational requirements.

The main motivation of this thesis has been to contribute to the acoustic diffusion equation model by presenting a general sound energy propagation theory that unifies its foundations with other geometrical acoustics methods and clarifies the needed assumptions for the model to be derived, as seen in Chap. 4.

Considering the practical issues, in Chap. 5 an alternative implementation of the acoustic diffusion equation model is developed by means of finite-difference method in the time domain to directly predict the energy decay curve.

Using these numerical implementations in Chap. 6 several evaluations of the performance, advantages and limitations of the acoustic diffusion model are carried out in different scenarios.

\subsection{Contributions of this thesis}

In this thesis, the main contributions can be highlighted as follows:

- This thesis proposes a theoretical model for room acoustics simulations based on the radiative transfer theory (see Sec. 4.4). The model is derived by utilizing the corresponding equations in optics, but tak- 
ing into account the acoustics quantities. The main contribution of the acoustic radiative transfer model is that expands classical geometrical room acoustics modelling algorithms incorporating a propagation medium that can scatter energy, as well as absorb.

- Although the presented acoustic radiative transfer model is purely theoretical, it unifies the theoretical basis for the geometrical acoustics models. Moreover, a connection to room acoustics simulation is established by showing that two well-known theoretical models, the diffusion equation model and the room-acoustic rendering equation, are special cases of the proposed model.

- A detailed derivation of those models is provided in Sec. 4.5 and Sec. 4.6. This derivation is a significant contribution in that it unifies these models and frames their limitations. A discussion about assumptions, advantages and disadvantages of each model is presented.

- The derivation of the diffusion equation is particularly of relevance consolidating its features and clarifying its foundations.

- A deep analysis of finite-difference methods for implementing the acoustic diffusion equation model is presented. Numerical solutions both the governing equation and the boundary conditions of two implicit schemes, FTCS and DF are derived. Their stability condition including the air absorption effect are obtained. A discussion about their suitability for efficient 3-D simulations is presented.

- An optimal finite-difference numerical solution for room acoustics simulation in 3-D is developed in Sec. 5.2.3 through the Dufort-Frankel scheme offering an easy and flexible algorithm with no stability restrictions, a second-order error and low computational requirements (see Sec. 5.3).

- The applicability of the diffusion equation model for room acoustics simulations is investigated using the proposed discrete-time imple- 
mentations. The performance of the model is analysed by comparing predicted values in both rectangular rooms in Sec. 6.2 and complex rooms in Sec. 6.3 with measured data and predictions from other geometrical methods.

- The simulations performed as regards to the accuracy in the temporal (in Sec. 6.2.3) and spatial domain (in Sec. 6.3.2) of the predicted sound field distribution are of particular importance.

Some parts of this program of research have been presented previously, at conferences and in journal papers. These publications are listed as follows:

\section{Journal articles}

- J. M. Navarro, F. Jacobsen, J. Escolano, and J. J. López, "A theoretical approach to room acoustic simulations based on a radiative transfer model", Acta Acust. united with Ac., 96(6), pp. 1078-1089, Nov/Dec, 2010.

- J. Escolano, J. M. Navarro and J. J. López, "On the limitation of a diffusion equation model for acoustic predictions of rooms with homogeneous dimensions (L)", J. Acoust. Soc. Am., 128(4),pp. 1586-1589, Oct, 2010.

- J. M. Navarro, J. Escolano, and J. J. López, "Implementation and evaluation of a diffusion equation model based on finite difference schemes for sound field predictions in rooms", Appl. Acoust., (inpress).

\section{Conference articles}

- J. M. Navarro, J. Escolano and J. J. López, "A physically-based model for fast prediction of the reverberation time in room acoustic applications", EAA Symposium on Auralization, Espoo, Finland, June 2009. 
- J. M. Navarro, J. Escolano and J. J. López, "A preliminary comparative investigation between a diffusion equation model and diffuse reflection algorithms in room-acoustics modeling", 4th International Symposium on Communications, Control and Signal Processing (ISCCSP'10), Limassol, Cyprus, March 2010.

- J. M. Navarro, J. Escolano and J. J. López, "On the air absorption effects in a finite difference implementation of the acoustic diffusion equation model", 128th AES Convention London, UK, May 2010.

- J. Escolano, J. M. Navarro, D. T. Murphy, J. J. Wells, and J. J. López, "A comparison of two diffuse boundary models based on finite differences schemes", 128th AES Convention London, UK, May 2010.

- J. M. Navarro, J. Escolano and J. J. López, "Some preliminary comparisons between the diffusion equation model and the room-acoustic rendering equation in complex scenarios", 130th AES Convention, London, UK, May 2011.

\subsection{Future research lines}

From the conclusions of this work, some new and challenging research lines could be proposed, some of them being already open. Future work may follow the lines listed here:

- Acoustic radiative transfer equation: Since this thesis introduced a theoretical approach for acoustic energy propagation within rooms, the next step will be to implement a numerical solution and to evaluate the performance of this algorithm in several scenarios. Systematic experiments should be carried out to analyse how reliable the acoustic radiative transfer equation is to be used directly in room acoustics simulations. 
- Compare the performance of the acoustic radiative transfer equation with its specializations: Although the diffusion equation and the rendering equation models are specializations of the acoustic radiative transfer equation and some analysis to verify their accuracy have been already presented, it is of relevance to compare these three methods between them to clarify their relationship in terms of accuracy, computational cost and applicability.

- High-order approximation of the acoustic radiative transfer equation: The high computational cost involved in the solution of the integrodifferential equation can be decreased if simplifications are made. To develop other particularisations of this geometrical model would be of interest. For instance, a high-order approximation using spherical harmonics is possible.

- Study the pros and cons of applying finite element methods and finitedifference methods to solve the acoustic diffusion equation model. A systematic comparison between them in different complexity scenarios in terms of accuracy, computational cost and applicability is of interest.

- Frequency-dependent boundary conditions in the diffusion equation model: To investigate the performance of the existing boundary conditions and analyse possible optimization. Besides, frequency-dependent boundary conditions in the discrete method could be proposed, in order to obtain a more efficient method.

- Hybrid simulation method: Recently, many studies are emerging which seek to combine the advantages of several simulation methods to obtain more efficient, realistic and accurate results [Southern et al., 2011]. The acoustic diffusion equation implementation developed in this thesis could be an adequate method used to create an hybrid method because it uses finite-difference schemes as the wave-based finite-difference time-domain method. The diffusion equation model 
should be used in the high-frequency band and the wave-based model in the low-frequency band prediction. Another expansion can be to compose with another geometrical model, e.g image-source method, a prediction of the early part of the impulse response in the highfrequency band.

- Auralisation: To perform psychoacoustic evaluations of computed room acoustics which include a time hybrid method. The final aim of these simulation algorithms is to provide a psychoacoustic acceptable result. This can be investigated and evaluated, focusing on psychoacoustic and subjective aspects to find fast computational solutions with sufficiently accurate results. 
$\oplus$

$\bigoplus$

$\oplus$ 


\section{Bibliography}

International standard 354, "acoustics - measurement of sound absorption in a reverberation room", 2003.

International standard 17497-1, "acoustics - measurement of the sound scattering properties of surfaces", 2004.

International standard 3382, "acoustics - measurement of reverberantion time of rooms with reference to other acoustical parameters", 2009.

S. Adams, J. Payne, and R. Boppana. Finite difference time domain (fdtd) simulations using graphics processors. HPCMP Users Group Conference, 0:334-338, 2007.

W. Ahnert, M. Bansal, and S. Feistel. Large scale FEM analysis of a studio room by. In Proc. Audio Eng. Soc. Convention, pages Paris, France, 2006.

D. Alarcáo and J. L. Bento. A general model for the propagation of sound energy inside enclosures. In Proc. 12th International Congress on Sound and Vibration (ICS V12), Lisbon, Portugal, July 2005.

J. B. Allen and D. A. Berkley. Image method for efficiently simulating small-room acoustics. J. Acoust. Soc. Am., 65(4):943-950, 1979. 
J. C. Allred and A. Newhouse. Applications of the monte carlo method to architectural acoustics. J. Acoust. Soc. Am., 30 (1):1-3, 1958.

Y. Ando. Concert Hall Acoustics. Springer-Verlang, 1985.

L. Antani, A. Chandak, M. Taylor, and D. Manocha. Direct-to-indirect acoustic radiance transfer. IEEE Transactions on Visualization and Computer Graphics, 99:1-1, 2011.

I. Ashdown. Radiosity: A programmer's perspective. John Wiley \& Sons, New York, 1994.

B. Atal, M. R. Schroeder, and G. Sessler. Subjective reverberation time and its relation to sound decay. In Proc. ICA, Liège, Belgium, G32, 1965.

M. R. Bai. Study of acoustic resonance in enclosures using eigenanalysis based on boundary element methods. J. Acoust. Soc. Am., 91:2529-2538, 1992.

C. A. Balanis. Antenna theory. John Wiley \& Sons, 1997.

M. Barron. Auditorium acoustics and architectural design. E\&FN Spon, London, 2000.

H. E. Bass, H. J. Bauer, and L. B. Evans. Atmospheric absorption of sound: Analytical expressions. J. Acoust. Soc. Am., 52(3):821-825, 1972.

L. L. Beranek. Acoustics. McGraw-Hill, 1954.

L. L. Beranek. Music, acoustics and architecture. J. Wiley, New York/London, 1962.

A. Billon, V. Valeau, A. Sakout, and J. Picaut. On the use of a diffusion model for acoustically coupled rooms. J. Acoust. Soc. Am., 120 (4):20432054, 2006.

A. Billon, C. Foy, J. Picaut, V. Valeau, and A. Sakout. Modeling the sound transmission between rooms coupled through partition walls by using a diffusion model. J. Acoust. Soc. Am., 123 (6):4261-4271, 2008a. 
A. Billon, J. Picaut, C. Foy, V. Valeau, and A. Sakout. Introducing atmospheric attenuation within a diffusion model for room-acoustic predictions. J. Acoust. Soc. Am., 123 (6):4040-4043, 2008b.

A. Billon, J. Picaut, and A. Sakout. Prediction of the reverberation time in high absorbent room using a modified-diffusion model. Appl. Acoust., 69:68-74, 2008c.

B. Blesser. An interdisciplinary synthesis of reverberation viewpoints. $J$. Audio Eng. Soc., 49 (10):867-903, 2001.

C. Borel, S. A. Gerstl, and B. J. Powers. The radiosity method in optical remote sensing of structured 3-d surfaces. Remote Sensing of the Environment, 36:13-44, 1991.

J. Borish. Extension of the image model to arbitrary polyhedra. J. Acoust. Soc. Am., 75(6):1827-1836, 1984.

I. Bork. A comparison of room simulation software - the 2nd round robin on room acoustical computer simulation. Acta Acust. united Ac., 86: 943-956, 2000.

I. Bork. Report on the 3rd round robin on room acoustical computer simulation - part i: measurements. Acta Acustica united with Acustica, 91: $740-752,2005 \mathrm{a}$.

I. Bork. Report on the 3rd round robin on room acoustical computer simulation - part ii: calculations. Acta Acustica united with Acustica, 91: 753-763, 2005b.

D. Botteldooren. Finite-difference time-domain simulation of low-frequency room acoustic problems. J. Acoust. Soc. Am., 98(6):3302-3308, Dec. 1995.

D. Botteldooren. Time-domain simulation of the influence of close barriers on sound propagation to the environment. J. Acoust. Soc. Am., 101: 1278-1285, 1997. 
M. M. Carroll and C. F. Chien. Decay of reverberant sound in a spherical enclosure. J. Acoust. Soc. Am., 62:1442-1446, 1977.

M. F. Cátedra, J. Pérez, F. S. de Adana, and O. Gutiérrez. Efficient raytracing techniques for three-dimensional analyses of propagation in mobile communications: Application to picocell ands microcell scenarios. IEEE Antennas and Propagation Magazine, 40(2):15-28, 1998.

S. Chandrasekar. Radiative transfer. Dover Publications, New York, 1960.

G. Chen and J. Zhou. Boundary Element Methods. Academic Press, 1992.

M. Cohen and J. R. Wallace. Radiosity and realistic image synthesis. Academic Press, Inc., Boston, 1993.

M. F. Cohen, S. E. Chen, J. R. Wallace, and D. P. Greenberg. A progressive refinement approach to fast radiosity image creation. Comput. Graph., $22: 75-84,1988$.

C. Coleman. A ray tracing formulation and its application to some problems in over-the-horizon radar. Radio Science, 33:1187-1197, 1998.

F. Cotana. An improved room acoustics model. Appl. Acoust., 61:1-25, 2000.

R. Courant. Variational methods for the solution of problems of equilibrium and vibrations. Bull. Am. Math. Soc., 49:1-23, 1943.

T. J. Cox, B.-I. L. Dalenbäck, P. D’Antonio, J. J. Embrechts, J. Y. Jeon, E. Mommertz, and M. Vorländer. A tutorial on scattering and diffusion coefficients for room acoustic surfaces. Acta Acust. united Ac., 92:1-15, 2006.

J. Crank and P. Nicolson. A practical method for numerical evaluation of solution of partial differential equations of the heat conduction type. Proc. Camb. Phil. Soc., 43:50-67, 1974. 
D. R. Croft and D. G. Lilley. Heat transfer calculations using finite difference equations. Applied Science Publishers LTD, 1977.

B.-I. L. Dalenbäck. Room acoustic prediction based on a unified treatment of diffuse and specular reflection. J. Acoust. Soc. Am., 100(2):899-909, 1996.

B.-I. L. Dalenbäck, P. Svensson, and M. Kleiner. Room acoustic prediction and auralization based on an extended image source model. J. Acoust. Soc. Am., 92 (4):2346-2346, 1992.

B.-I. L. Dalenbäck, M. Kleiner, and P. Svensson. A macroscopic view of diffuse reflection. J. Audio Eng. Soc., 42(10):973-807, 1994.

G. Defrance and J.-D. Polack. Estimating the mixing time of concert halls using the extensible fourier transform. Applied Acoustics, 71:777-792, 2010 .

J. Dominguez. Boundary Elements in Dynamics. Elsevier Applied Science, 1993.

E. C. Dufort and S. P. Frankel. Stability conditions in the numerical treatment of parabolic differential equations. Mathematical tables and others aids to computation, 7:135-152, 1953.

S. A. V. Duyne and J. O. Smith. Physical modeling with the 2-D digital waveguide mesh. In Proc. of the 1993 International Computer Music Conference, Waseda University, Japan, 1993.

S. A. V. Duyne and J. O. Smith. The 3-D tetrahedral digital waveguide mesh. In Proc. of the IEEE Workshop on Applications of Signal Processing of Audio and Acoustics, New Platz, NY, 1995.

J. J. Embrechts. Broad spectrum diffusion model for room acoustics raytracing algorithms. J. Acoust. Soc. Am., 107(4):2068-2081, 2000. 
J. Escolano and J. J. López. Broadband directive sources modeling for acoustic discrete-time domain methods. J. Acoust. Soc. Am., 126 (6): 2856-2859, 2009.

J. Escolano, F. Jacobsen, and J. J. López. An efficient realization of frequency dependent boundary conditions in a finite-difference time-domain model. J. Sound Vibration, 316:234-247, 2008.

J. Escolano, J. J. López, and B. Pueo. Locally reacting impedance model in a digital waveguide mesh by mixing modeling strategies for room acoustic simulation. Acta Acust. united Ac., 95 (6):1048-1059, 2009.

J. Escolano, J. M. Navarro, and J. J. Lopez. On the limitation of a diffusion equation model for acoustic predictions of rooms with homogeneous dimensions (1). J. Acoust. Soc. Am., 128:1586-1589, 2010.

F. A. Everest. Master Handbook of Acoustics. McGraw-Hill, New York, 2001.

C. F. Eyring. Reverberation time in ŞdeadŤ rooms. J. Acoust. Soc. Am., 1 (2A):168, 1930.

A. Farina. RAMSETE - a new pyramid tracer for medium and large scale acoustic problems. In Proc. Euro-Noise 95, March 1995.

C. Foy, V. Valeau, A. Billon, J. Picaut, and A. Sakout. An empirical diffusion model for acoustic prediction in rooms with mixted specular and diffuse refections. Acta Acust. united Ac., 95:97-105, 2009.

L. P. Franzoni and J. W. Rouse. An intensity-based boundary element method for analyzing broadband high frequency sound fields in enclosures. In Proceedings of the Forum Acusticum, Sevilla, Spain, September 2002. Spanish Acoustical Society.

T. Funkhouser, I. Carlbom, G. Elko, G. Pingali, M. Sondhi, and J. West. A beam tracing approach to acoustic modeling for interactive environments. 
In Proceedings of the 25th annual conference on Computer graphics and interactive techniques, pages 21-32, New York, USA, 1998. ACM Press.

T. Funkhouser, N. Tsingos, I. Carlbom, G. Elko, M. Sondhi, J. West, G. Pingali, P. Min, and A.Ngan. A beam tracing method for interactive architectural acoustics. J. Acoust. Soc. Am., 115(2):739-756, 2004.

A. J. Gandomi and H. Takenaka. Efficient FDTD algorithm for plane-wave simulation for vertically heterogeneous attenuative media. Geophysics, 72(4):H43-H53, July/August 2007.

E. D. Geest and H. Patzold. Comparison between room transmission functions calculated with a boundary element method and a ray tracing method including phase. In Proceedings of internoise 96, 1996.

S. A. Gerstl and C. C. Borel. Principles of the radiosity method versus radiative transfer for canopy reflectance modeling. IEEE transactions on geoscience and remote sensing, 30:271-275, 1992.

A. S. Glassner. An introduction to Ray Tracing. Academic Press, 1989.

C. M. Goral, K. E. Torrance, D. P. Greenberg, and B. Battaile. Modeling the interaction of light between diffuse surfaces. Computer Graphics, 18 (3):213-222, 1984.

R. W. Graves. Simulating seismic wave propagation in 3-D elastic media using staggered-grid finite differences. Bull. Seismol. Soc. Am., 86:1091$106,1996$.

J. H. Halton. A retrospective and prospective of the Monte Carlo method. SIAM Review, 12(1):1-63, Jan 1970.

R. C. Haskell, L. O. Svaasand, T. Tsay, T. F. M. S. McAdams, and B. J. Tromberg. Boundary conditions for the diffusion equation in radiative transfer. J. Opt. Soc. Am., 11:2727Û́2741, 1994. 
R. C. Heyser. Acoustical measurements by time delay spectrometry. $J$. Audio Eng. Soc., 15:370-382, 1967.

M. Hodgson. On the prediction of sound fields in large empty rooms. $J$. Acoust. Soc. Am., 84 (1):253-261, 1988.

M. Hodgson. Case history: Factory noise prediction using ray tracing experimental validation and the effectiveness of noise control measures. Noise Control Engineering Journal, 33(3):97-104, 1989.

M. Hodgson. On the accuracy of models for predicting sound propagation in fitted rooms. J. Acoust. Soc. Am., 88(2):871-878, 1990.

M. Hodgson. Evidence of diffuse surface reflection in rooms. J. Acoust. Soc. Am., 89 (2):765-771, 1991.

M. Hodgson. When is diffuse-field theory applicable? Appl. Acoust., 49(3): 197-207, 1996a.

M. Hodgson. Sound-propagation curves in industrial workrooms: statistical trends and empirical prediction models. J. Building Acoust., 3(1):25-32, 1996b.

M. Hodgson. Experimental evaluation of simplified models for predicting noise levels in industrial workrooms. J. Acoust. Soc. Am., 103(4):19331939, 1998.

M. Hodgson and E.-M. Nosal. Experimental evaluation of radiosity for room sound-field prediction. J. Acoust. Soc. Am., 120:808-819, 2006.

D. Howard and J. Angus. Acoustics and Psychoacoustics. Focal Press, 1996.

M. J. Howarth and Y. W. Lam. An assessment of the accuracy of a hybrid room acoustics model with surface diffusion facility. Appl. Acoust., 60: 237-251, 2000.

J. R. Howell. The monte carlo method in radiative heat transfer. Journal of Heat Transfer, 120 (3):547-560, 1998. 
F. Ihlenburg. Finite Element Analysis of Acoustic Scattering. SpringerVerlag, New York, USA, 1998.

A. Ishimaru. Wave Propagation and Scattering in Random Media. Academic, New York, 1978.

H. Iwabuchi. Efficient monte carlo methods for radiative transfer modeling. Journal of the Atmospheric Sciences, 63 (9):2324-2339, 2006.

F. Jacobsen. Handbook of Acoustics, chapter Sound intensity, pages 10531075. Springer, 2007.

Z. Ji, B.-H. Li, H.-X. Wang, H.-Y. Chen, and T. K. Sarkar. Efficient raytracing methods for propagation prediction for indoor wireless communications. IEEE Antennas and Propagation Magazine, 43(2):41-49, 2001.

J. Jin. The Finite Element Method in Electromagnetics. Wiley-IEEE Press, 2002.

Y. Jing and N. Xiang. A modified diffusion equation for room-acoustic predication (1). J. Acoust. Soc. Am., 121:3284-3287, 2007.

Y. Jing and N. Xiang. On boundary conditions for the diffusion equation in room acoustic predictions: Theory, simulations, and experiments. $J$. Acoust. Soc. Am., 123:145-153, 2008.

Y. Jing and N. Xiang. One-dimensional transport equation models for sound energy propagation in long spaces: Simulations and experiments. J. Acoust. Soc. Am., 127 (4):2323-2331, 2010.

Y. Jing, E. W. Larsen, and N. Xiang. One-dimensional transport equation models for sound energy propagation in long spaces: Theory. J. Acoust. Soc. Am., 127 (4):2312-2322, 2010.

W. Joyce. Classical-particle description of photons and phonons. Physical Review D, 9:3234-3256, 1974. 
J. T. Kajiya. The rendering equation. Computer Graphics, 20:143-150, 1986.

J. Kang. Reverberation in rectangular long enclosures with diffusely reflecting boundaries. Acust. Acta Acust., 88:77-87, 2002.

G. Kanschat. Numerical Methods in Multidimensional Radiative Transfer. Springer, Berlin, Germany, 2008.

L. E. Kinsler, A. R. Frey, A. B. Coppens, and J. V. Sanders. Fundamentals of Acoustics. John Wiley \& Sons, New York, 4th edition, 2000.

M. Kleiner, P. Svensson, and B.-I. Dalenbäck. Auralization: Experiments in acoustical CAD. In Proc. of the 89th Audio Eng. Soc. Convention, Los Angeles, USA, 1990.

M. Kleiner, B.-I. L. Dalenbäck, and P. Svensson. Auralization - an overview. J. Audio Eng. Soc., 41(11):861-875, November 1993.

N. Korany, J. Blauert, and O. A. Alim. Acoustic simulation of rooms with boundaries of partially specular reflectivity. Appl. Acoust., 62:875-887, 2001.

C. W. Kosten. The mean free path in room acoustics. Acustica, 10:245-250, 1960.

K. Kowalczyk and M. van Walstijn. Room acoustics simulation using 3-d compact explicit fdtd schemes. IEEE Transactions on Audio, Speech, and Language Processing, 19 (1):34-46, 2011.

K. Kowalczyk, M. van Walstijn, and D. Murphy. A phase grating approach to modeling surface diffusion in fdtd room acoustics simulations. IEEE Transactions on Audio, Speech, and Language Processing, 19 (3):528-537, 2011.

J. Kristek and P. Moczo. On the accuracy of the finite-difference schemes: The 1-D elastic problem. Seismological Soc. Am., 96:2398-2414, 2006. 
A. Krokstad, S. Strom, and S. Sorsdal. Calculating the acoustical room response by the use of a ray tracing technique. J. Sound Vibration, 8(1): 118-125, 1968.

A. Kulowski. Algorithmic representation of the ray tracing technique. Appl. Acoust., 18(6):449-469, 1985.

K. S. Kunz and R. J. Luebbers. The Finite Difference Time Domain Method for Electromagnetics. CRC Press, 1993.

U. J. Kurze. Scattering of sound in industrial spaces. Journal of Sound and Vibrations, 98:349-364, 1985.

H. Kuttruff. Simulated reverberation curves in rectangular rooms with diffuse sound fields. Acustica, 25 (6):333-342, 1971.

H. Kuttruff. Reverberation and effective absorption in rooms with diffuse wall reflections. Acustica, 35 (3):141-153, 1976.

H. Kuttruff. Sound propagation in working environments. In Proc. 5th FASE symposium, Thessaloniki, 1985.

H. Kuttruff. Auralization of impulse responses modeled on the basis of ray-tracing results. J. Audio Eng. Soc., 41(11):876-880, 1993.

H. Kuttruff. An iterative scheme for the computation of decay constants in enclosures with diffusely reflecting surfaces. J. Acoust. Soc. Am., 98 (1): 288-293, 1995.

H. Kuttruff. Energetic sound propagation in rooms. Acust. Acta Acust., 83: 622-628, 1997.

H. Kuttruff. Stationary propagation of sound energy in flat enclosures with partially diffuse surface reflection. Acta Acustica, 86:1028-1033, 2000.

H. Kuttruff. Room Acoustics. Taylor \& Francis, 4th edition, 2000. 
Y. W. Lam. A comparison of three diffuse reflection modeling methods used in room acoustics computer models. J. Acoust. Soc. Am., 100(4): 2181-2192, 1996.

A. LeBot. A functional equation for the specular reflection of rays. $J$. Acoust. Soc. Am., 112 (4):1276-1287, 2002.

H. Lehnert and J. Blauert. Principles of binaural room simulation. Appl. Acoust., 36:259-291, 1992.

H. Levy and F. Lessman. Finite difference equations. Dover books on advanced mathematics. Dover Publications, 1992.

T. Lewers. A combined beam tracing and radiant exchange computer model of room acoustics. Appl. Acoust., 38:161-178, 1993.

S. G. Lipson, H. Lipson, and D. S. Tannhauser. Optical Physics. Cambridge University Press, 1995.

L. Liu and D. Albert. Acoustic pulse propagation near a right-angle wall. J. Acoust. Soc. Am., 119(4):2073-83, 2006.

J. W. McJown and R. L. Hamilton. Ray-tracing as a design tool for radio networks. IEEE Network Magazine, 5(6):27-30, 1991.

R. N. Miles. Sound field in a rectangular enclosure with diffusely reflecting boundaries. J. Sound Vibration, 92 (2):203-226, 1984.

P. Moon. The Scientific Basis of Illuminating Engineering. McGraw Hill, New York, 1936.

P. M. Morse and H. Feshbach. Methods of Theoretical Physics. McGrawHill, New York, 1953.

P. M. Morse and K. U. Ingard. Theoretical Acoustics. McGraw-Hill, New York, 1968. 
D. T. Murphy. Digital waveguide mesh topologies in room acoustics modelling. PhD thesis, Dept. Electronics, University of York, UK, 2000.

J. M. Navarro, J. Escolano, and J. J. Lopez. On the air absorption effects in a finite difference implementation of the acoustic diffusion equation model. In Proc. of the 128th AES Convention, London, UK, 22-25 May 2010a.

J. M. Navarro, J. Escolano, and J. J. Lopez. A preliminary comparative investigation between a diffusion equation model and diffuse reflection algorithms in room-acoustics modeling. In Proceedings of the 4 th International Symposium on Communications, Control and Signal Processing, Cyprus, 2010b.

J. M. Navarro, F. Jacobsen, J. Escolano, and J. Lopez. A theoretical approach to room acoustic simulations based on a radiative transfer model. Acta Acustica United with Acustica, 96:1078-1089, 2010c.

J. M. Navarro, J. Escolano, and J. J. Lopez. Some preliminary comparisons between the diffusion equation model and the room-acoustic rendering equation in complex scenarios. In Proc. of the 130th AES Convention, London, UK, May 2011.

J. M. Navarro, J. Escolano, and J. J. Lopez. Implementation and evaluation of a diffusion equation model based on finite difference schemes for sound field predictions in rooms. Appl. Acoust., in-press, 2012.

G. Naylor. ODEON - another hybrid room acoustical model. Appl. Acoust., 38:131-143, 1993.

M. Necati. Finite difference methods in heat transfer. CRC Press, 1994.

B. Nguyen. Investigation of Three-Level Finite-Difference Time-Domain Methods for Multidimensional Acoustics and Electromagnetics. PhD thesis, University of Michigan, 1996. 
E.-M. Nosal. Room sound field prediction by acoustical radiosity. Master's thesis, University of British Columbia, 2003.

E.-M. Nosal, M. Hodgson, and I. Ashdown. Investigation of the validity of radiosity for sound-field prediction in cubic rooms. J. Acoust. Soc. Am., 116:3505-3514, 2004a.

E.-M. Nosal, M. Hodgson, and I. Ashdown. Improved algorithms and methods for room sound-field prediction by acoustical radiosity in arbitrary polyhedral rooms. J. Acoust. Soc. Am., 116:970-980, $2004 \mathrm{~b}$.

F. Ollendorff. Statistical room-acoustics as a problem of diffusion: a proposal. Acustica, 21:236-245, 1969.

A. M. Ondet and J. L. Barbry. Modeling of sound propagation in fitted workshops using ray-tracing. J. Acout. Soc. Am., 85 (2):787-796, 1989.

A. V. Oppenheim, A. S. Willsky, and S. Hamid. Signals and Systems. Prentice Hall, 2nd edition edition, 1996.

T. Osa, K. Murakamib, Y. Horinouchia, and D. Takahashia. Application of audience-seats characteristics to the sound field analysis for large enclosures. Appl. Acoust., 68(9):939-952, 2006.

V. Ostashev, D. Wilson, L. Liu, D. Aldridge, N. Symons, and D. Marlin. Equations for finite-difference time-domain simulation of sound propagation in moving inhomogeneous media and numerical implementation. $J$. Acoust. Soc. Am., 117(2):503-17, 2005.

J. Picaut, L. Simon, and J.-D. Polack. A mathematical model of diffuse sound field based on a diffusion equation. Acta Acust. united Ac., 83: 614-621, 1997.

J. Picaut, L. Simon, and J. Hardy. Sound field modeling in streets with a diffusion equation. J. Acoust. Soc. Am., 106:2638-2645, 1999a. 
J. Picaut, L. Simon, and J.-D. Polack. Sound field in long rooms with diffusely reflecting boundaries. Appl. Acoust., 56:217-240, 1999b.

A. D. Pierce. Acoustics, An Introduction to Its Physical Principles and Applications. Acoustical Society of America, 1994.

A. D. Pierce. Handbook of Acoustics, chapter Basic linear acoustics, pages 25-112. Springer, 2007.

A. Pietrzyk. Computer modeling of the sound field in small rooms. In Audio Engineering Society Conference: 15th International Conference: Audio, Acoustics \&f Small Spaces, Octuber 1998.

A. Pietrzyk and M. Kleiner. The application of the finite-element method to the prediction of soundfields of small rooms at low frequencies. In Proc. 102th Audio Eng. Soc. Convention, pages Munich, Germany, 1997.

J.-D. Polack. Playing billiards in a concert hall: the mathematical foundations of geometrical acoustics. Applied Acoustics, 38:235-244, 1992.

T. L. Pollès, J. Picaut, M. Bérengier, and C. Bardos. Sound field modeling in a street canyon with partially diffusely reflecting boundaries by the transport theory. J. Acoust. Soc. Am., 116 (5):2969-2983, 2004.

T. L. Pollès, J. Picaut, S. Colle, and M. Bérengier. Sound-field modeling in architectural acoustics by a transport theory: Application to street canyons. Phys. Rev. E, 72 (4):046609, 2005.

R. W. Preisendorfer. Radiative transfer on Discrete Spaces. Pergamon Press, New York, 1965.

N. Raghuvanshi, R. Narain, and M. C. Lin. Efficient and accurate sound propagation using adaptive rectangular decomposition. IEEE Transactions on Visualization and Computer Graphics, 15 (5):789-801, 2009.

B. J. W. S. Rayleigh and R. B. Lindsay. The Theory of Sound, volume II. Reprinted Dover Publications, New York, 1945. 
J. Redondo, R. Picó, B. Roig, and M. Avis. Time domain simulation of sound diffusers using finite-difference schemes. Acta Acust. united Ac., 93 (4)(4):611-622, 2007.

W. Reichardt, O. A. Alim, and W. Schmidt. Abhängigkeit der grenzen zwischen brauchbarer und unbrauchbarer durchsichtigkeit von der art des musikmotives, der nachhallzeit und der nachhalleinsatzzeit. Appl. Ac., 7: 243, 1974 .

L. F. Richardson. The approximate arithmetical solution by finite differences of physical problems involving differential equations, with applications to the stresses in a masonry dam. Philos. Trans. R. Soc. London, 210:307-357, 1910 .

D. D. Rife and J. Vanderkooy. Transfer-function measurements using maximum-length sequences. J. Audio Eng. Soc., 37(6):419-444, 1989.

J. H. Rindel. Computer simulation techniques for acoustical design of rooms. Acoustics Australia, 23:81-86, 1995.

J. H. Rindel. The use of computer modelling in room acoustics. Journal of Vibroengineering, 3(4):219-224, 2000.

W. M. Rohsenow, J. P. Hartnett, and Y. I. Cho. Handbook of heat transfer. McGraw Hill, New York, 1998.

W. C. Sabine. Collected Papers on Acoustics. Dover Publications Inc, New York, 1964.

L. Savioja. Modeling Techniques for Virtual Acoustics. PhD thesis, Helsinki University of Technology, Telecommunications Software and Multimedia Laboratory, Espoo, Finland, 1999.

L. Savioja, T. J. Rinne, and T. Takala. Simulation of room acoustics with a 3-D finite difference mesh. In Proceedings of the International Computer Music Conference, pages 463-466, Aarhus, Denmark, 1994. 
L. Savioja, J. Backman, A. Järvinen, and T. Takala. Waveguide mesh method for low-frequency simulation of room acoustic. In Proc. 15th Int. Congr. Acoust. (ICA'95), volume 2, pages 637-640, June 1995. Trondheim, Norway.

L. Savioja, A. Järvinen, K. Melkas, and K. Saarinen. Determination of the low frequency behaviour of an IEC listening room. In Proc. of the Nordic Acoustical Meeting, pages 55-58, Helsinki, Finland, 1996a.

L. Savioja, M. Karjalainen, and T. Takala. DSP formulation of a finite difference method for room acoustics simulation. In Proc. IEEE Nordic Signal Processing Symp. (NORSIG'96), pages 455-458, Espoo, Finland, Sept 1996b.

L. Savioja, J. Huopaniemi, T. Lokki, and R. Väänänen. Creating interactive virtual acoustic environments. J. Audio Eng. Soc., 47(9):675-705, 1999.

L. Savioja, D. Manocha, and M. C. Lin. Use of gpus in room acoustic modeling and auralization. In International Symposium on Room Acoustics, ISRA 2010, 2010.

M. R. Schroeder. Natural-sounding artificial reverberation. J. Audio Eng. Soc., 10(3):219-223, 1962.

M. R. Schroeder. New method of measuring reverberation time. J. Acoust. Soc. Am., 37:409-412, 1965.

M. R. Schroeder. Computer models for concert hall acoustics. Am. J. Phys., 41:461-471, 1973.

M. R. Schroeder and K. H. Kuttruff. On the frequency response curves in rooms. comparison of experimental, theoretical and monte carlo results for the average frequency spacing between maxima. J. Acoust. Soc. Am., 34:76-80, 1962.

H.-C. Shin and J.-G. Ih. Acoustic analysis of interior spaces by using the phased geometric acoustic model. J. Acoust. Soc. Korea, 17:54-61, 1998. 
R. Siegel and J. R. Howell. Thermal Radiation Heat Transfer. Hemisphere Publishing Corp, New York, 2nd edition, 1981.

F. X. Sillion and C. Puech. Radiosity and global illumination. Morgan Kaufmann, San Francisco, 1994.

S. Siltanen and T. Lokki. Diffraction modeling in acoustic radiance transfer method. In Acoustics'08, pages 5625-5630, Paris, France, June 29-July 4 2008.

S. Siltanen, T. Lokki, S. Kiminki, and L. Savioja. The room acoustic rendering equation. J. Acoust. Soc. Am., 122(3):1624-1635, 2007 a.

S. Siltanen, T. Lokki, and L. Savioja. Acoustic radiance transfer method for room acoustic modeling. In the 19th International Congress on Acoustics (ICA'2007), number RBA-05-008, Madrid, Spain, September 2007b.

S. Siltanen, T. Lokki, and L. Savioja. Frequency domain acoustic radiance transfer for real-time auralization. Acta Acustica united with Acustica, 95:106-117, 2009.

S. Siltanen, T. Lokki, and L. Savioja. Room acoustics modeling with acoustics radiance transfer. In Proc. Int. Symposium on Room Acoustics, Melbourne, Australia, Aug. 2010.

S. Siltanen, T. Lokki, and L. Savioja. Efficient acoustic radiance transfer method with time-dependent reflections. In POMA - 161st Meeting Acoustical Society of America, 2011.

G. D. Smith. Numerical solution of partial differential equations: finite difference methods. Oxford University Press, 2nd edition, 1978.

A. Southern, D. Murphy, G. Campos, and P. Dias. Finite difference room acoustic modelling on a general purpose graphics processing unit. In Audio Engineering Society Convention 128, May 2010. 
A. Southern, S. Siltanen, and L. Savioja. Spatial room impulse responses with a hybrid modeling method. In Audio Engineering Society Convention 130, 2011.

U. Stephenson. Comparison of the image source method and the particle simulation method. Appl. Acoust., 29:35-72, 1990.

U. Stephenson. Quantized pyramidal beam tracing - a new algorithm for room acoustics and noise inmission prognosis. Acustica united with Acta Acustica, 82:517-525, 1996.

J. C. Strikwerda. Finite difference schemes and partial differential equations. Wadsworth \& Brooks, Pacific Grove, CA, 1989.

U. P. Svensson, R. I. Fred, and J. Vanderkooy. Analytic secondary source model of edge diffraction impulse responses. J. Acoust. Soc. Am., 106(5): 2331-2344, Nov 1999

A. Taflove. Review of the formulation and applications of the finitedifference time-domain method for numerical modeling of electromagnetic wave interactions with arbitrary structures. Wave Motion, 10:547-582, 1988 .

A. Taflove. Computational Electrodynamics: The Finite-Difference TimeDomain Method. Artech House, Norwood, MA, 1995.

A. Taflove. Advances in Computational Electrodynamics: The FiniteDifference Time-Domain Method. Artech House, Norwood, MA, 1998.

P. J. Taylor. The stability of the du fort-frankel method for the diffusion equation with boundary conditions involving space derivatives. The Computer Journal, 13 (1):92-97, 1970.

J. F. Thompson, B. K. Soni, and N. P. Weatherill. Handbook of grid generation. CRC Press, 1999. 
J. K. Thompson, L. D. Mitchell, and C. J. Hurst. A modified room acoustics approach to determine sound-pressure levels in irregularly-proportioned workroom spaces. In Proc. Inter-Noise 'r6, 1976.

L. N. Trefethen. Group velocity in finite difference schemes. SIAM Review, 24:113-136, 1982.

N. Tsingos, I. Carlbom, G. Elko, R. Kubli, and T. Funkhouser. Validating acoustical simulations in the Bell Labs box. IEEE Computer Graphics and Applications, 22(4):28-37, Jul/Aug 2002.

V. Valeau, J. Picaut, A. Sakout, and A. Billon. Simulation of the acoustics of coupled rooms by numerical resolution of a diffusion equation. In 18th International Congress on Acoustics (ICA), Kioto, Japan, 2004. ICA, ICA.

V. Valeau, J. Picaut, and M. Hodgson. On the use of a diffusion equation for room-acoustic prediction. J. Acoust. Soc. Am., 119(3):1504-1513, 2006.

V. Valeau, M. Hodgson, and J. Picaut. A diffusion-based analogy for the prediction of sound fields in fitted rooms. Acta Acust. united Ac., 93: 94-105, 2007.

R. J. van der Geest and C. McChulloch. The virtual room: deriving acoustic characteristics by modelling. Proocedings of the Institute of Acoustics Reproduced Sound, 20:169-180, 1998.

M. Vorländer. International round robin on room acoustical computer simulation. In Proceedings of 15th International Congress on Acoustics, pages S.577-580, Trondheim, Norway, 1995.

M. Vorländer. Simulation of the transient and steady-state sound propagation in rooms using a new combined ray-tracing/image-source algorithm. J. Acoust. Soc. Am., 86(1):172-188, 1989.

M. Vorländer. Auralization: Fundamentals of Acoustics, Modelling, Simulation, Algorithms and Acoustic Virtual Reality. Springer, 2008. 
L. Wang and H. Wu. Biomedical Optics: Principles and Imaging. WileyInterscience, 2007.

S. Wang. Finite-difference time-domain approach to underwater acoustic scattering problems. J. Acoust. Soc. Am., 99:1924-1931, 1996.

T. Whitted. An improved illumination model for shaded display. Communications of the ACM, 23(6):343-349, 1980.

E. G. Williams. Fourier Acoustics. Academic Press, London, 1999.

L. B. Wolf. Diffuse reflection. In Proceedings of the IEEE Conference on Computer Vision and Pattern Recognition, volume 23, pages 472-478, 1992.

J. R. Wright. An exact model of acoustic radiation in enclosed spaces. J. Audio Eng. Soc., 43(10):813-820, 1995.

L. Wrobel and F. Aliabadi. The Boundary Element Method, Volume 1: Applications in Thermo-Fluids and Acoustics. John Wiley, New York, USA, 2002.

N. Xiang, Y. Jing, and A. Bockman. Investigation of acoustically coupled enclosures using a diffusion-equation model. J. Acoust. Soc. Am., 126: 1187-1198, 2009.

Z. Yamauti. The light flux distribution of a system of interreflecting surfaces. Journal of the Optical Society of America, 13 (5):561-571, 1926.

K. Yee. Numerical solution of initial boundary value problems involving Maxwell's equations in isotropic media. IEEE Trans. Antennas Propagat., 14:302-307, 1966. 